\title{
Controlled folding and conformational switching in metal-mediated DNA constructs
}

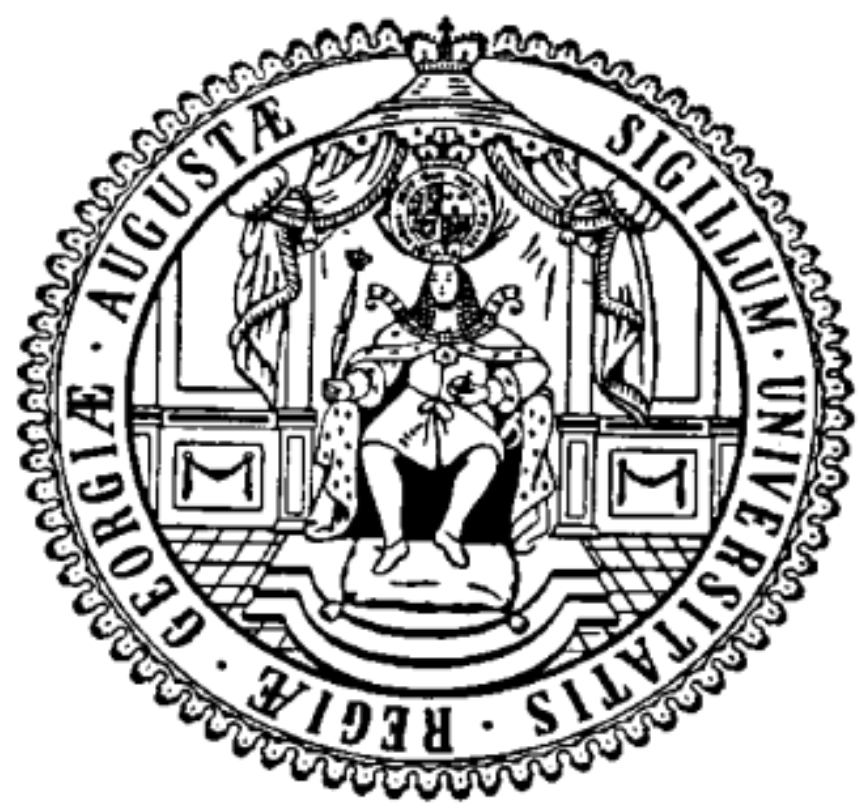

Dissertation for the award of the degree

"Doctor rerum naturalium" (Dr.rer.nat.) of

Georg-August-Universität Göttingen

Faculty of Chemistry

Institute of Inorganic Chemistry

Within the international Ph.D. program Metal Sites in Biomolecules:

Structures, Regulation and Mechanism (IRTG 1422)

Submitted by

Fernanda Antonia Pereira

From Belo Horizonte, Brazil

Göttingen, 2016 



\section{Thesis Commitee}

Prof. Dr. Guido H. Clever

Faculty of Chemistry and Chemical Biology, TU Dortmund University, Dortmund.

Prof. Dr. Franc Meyer

Institute of Inorganic Chemistry, Georg-August-Universitat, Gottingen

Prof. Dr. Sofi Elmroth

Biochemistry and Structure biology, Lund University, Lund

Members for the Examination Board

Reviewer

Prof. Dr. Guido H. Clever

Faculty of Chemistry and Chemical Biology, TU Dortmund University, Dortmund.

$2^{\text {nd }}$ Reviewer

Prof. Dr. Franc Meyer

Institute of Inorganic Chemistry, Georg-August-Universitat, Gottingen

Prof. Dr. Philipp Vana

Institute of Physical Chemistry, Georg-August-Universitat, Gottingen

Dr. Alexander Breder

Institute of Organic and Biomolecular Chemistry, Georg-August-Universitat, Gottingen

Jun-Prof. Dr. Selvan Demir

Institute of Inorganic Chemistry, Georg-August-Universitat, Gottingen

Prof. Dr. Sven Schneider

Institute of Inorganic Chemistry, Georg-August-Universitat, Gottingen 



\section{Affidavit}

I hereby declare that my doctoral thesis entitled "Controlled folding and conformational switching in metal-mediated DNA constructs" has been written independently and with no other sources and aids than quoted. Parts performed by project collaborators were properly indicated.

Fernanda Antonia Pereira

Göttingen, August 2016 



\section{List of publications}

F. A. Pereira, M. Light, E. Stulz, G. H. Clever, Synthetic Introduction of Interduplex Cross-Links into DNA through a trans-Chelating Ligand for Square-Planar Metal Ions, manuscript in preparation.

F. A. Pereira, G. H. Clever, DNA incorporation of a trans-chelating bis-Pyridyl ligand for square-planar coordinated metal cations, Inorg. Chim. Acta 2016, in press.

F. A. Pereira, T. Fallows, M. Frank, A. Chen, G.H. Clever, Stable and Metastable Self-Assembled Rings based on trans-chelated Pd(II), Z. Anorg. Allg. Chem. 2013, 639, (8-9), 1598-1605. 



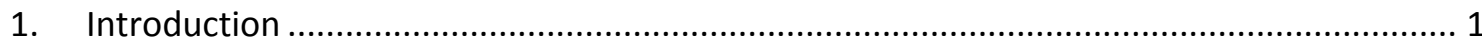

1.1. Structure and function of Deoxyribonucleic acid (DNA) ....................................... 1

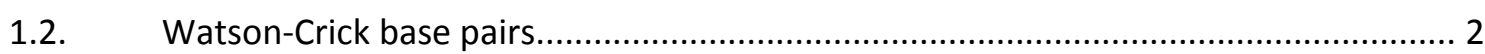

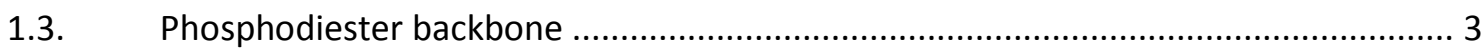

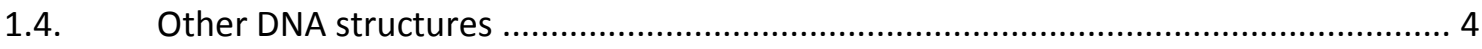

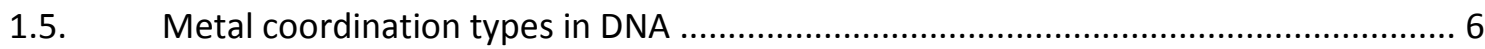

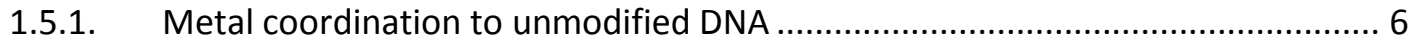

1.5.2. Metal coordination in modified DNA ................................................................ 9

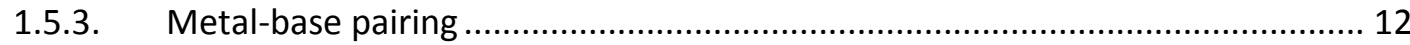

1.5.4. Inter cross-link between DNA duplexes.......................................................... 14

1.6. Theory for synthetic, analytic and spectroscopic methods .................................... 16

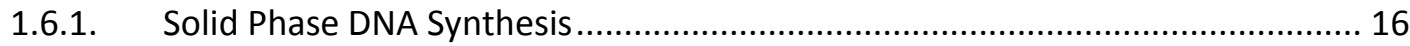

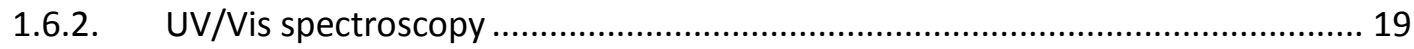

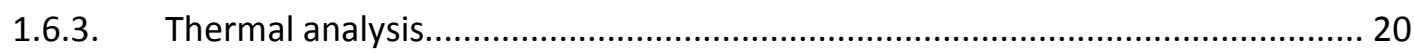

1.6.3.1. Determination of the melting point.......................................................... 21

1.6.3.2. Considerations about nucleation, zippering and the "all-or-none" model for

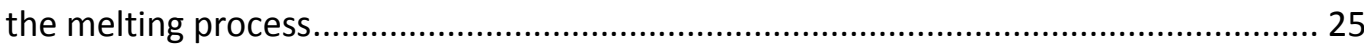

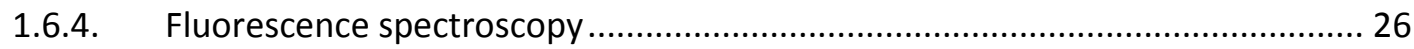

1.6.5. Fluorescence resonance energy transfer, FRET ............................................... 28

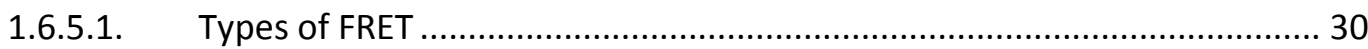

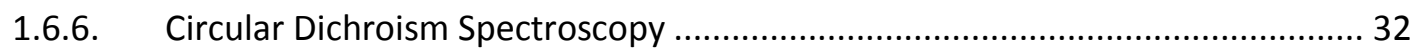

1.6.7. Electrospray ionization mass spectrometry, ESI-MS...................................... 33

1.6.8. Methods of purification for oligonucleotides .................................................... 34

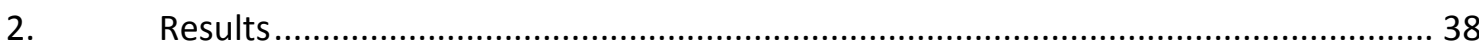

2.1. Aims of project (part 1) - Orthogonal self-assembly between trans-chelating, terpyridine, phenanthroline, other related ligands, and divalent metal species....................... 38

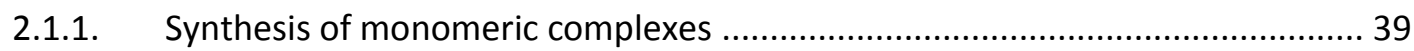

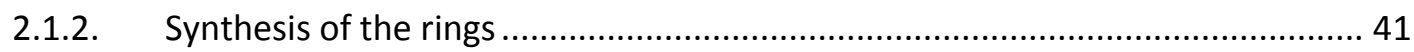

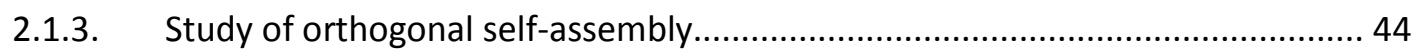

2.2. Aims of the project (part 2) - Metal Base Pairing based on trans-chelating ligand ... 48

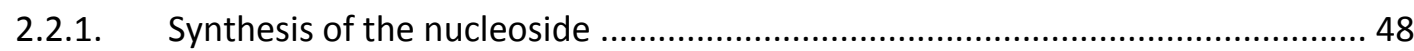

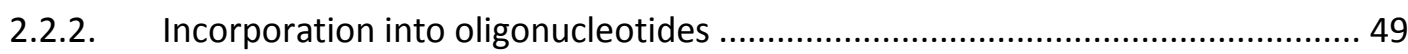


2.2.3. Sample conditions and preparation ............................................................. 54

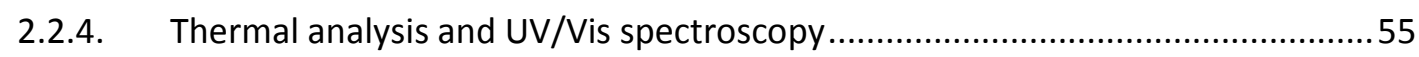

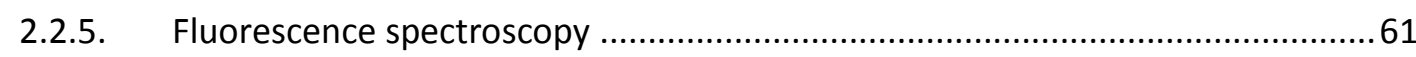

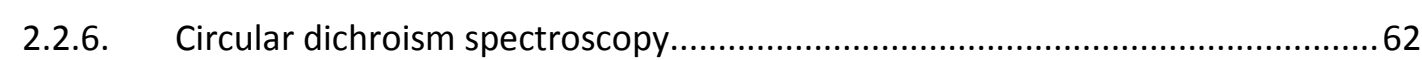

2.2.7. Electrospray ionization mass spectrometry for DNA duplex and metal-base

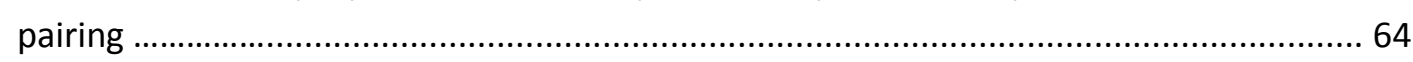

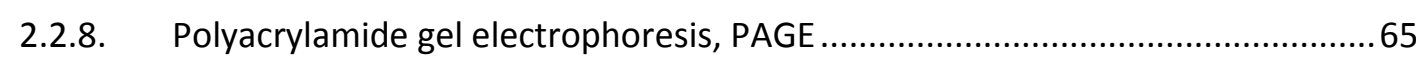

2.3. Aims of the project (Part 3) - Artificial trans-chelating nucleoside poiting outside of the DNA helix and DNA inter cross-link through metal complex formation 67

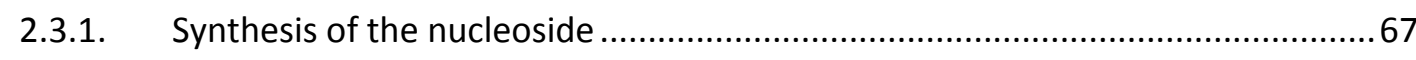

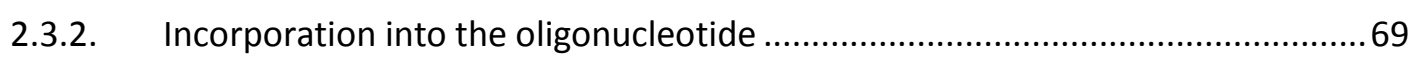

2.3.3. Results for the single modified system, s3.s2*............................................... 76

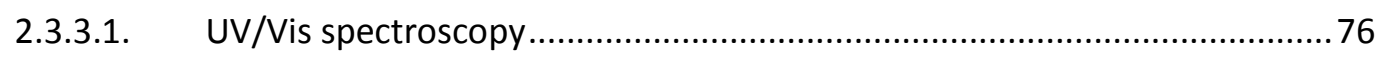

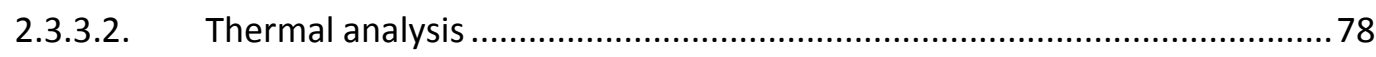

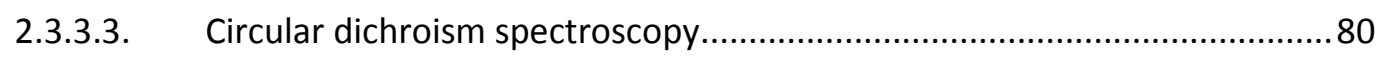

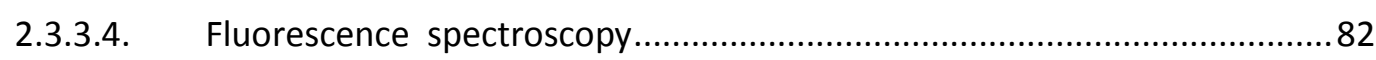

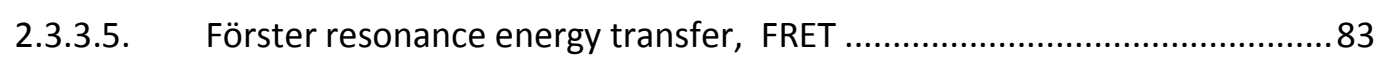

2.3.3.6. Electrospray ionization mass, ESI-MS spectrometry ................................... 85

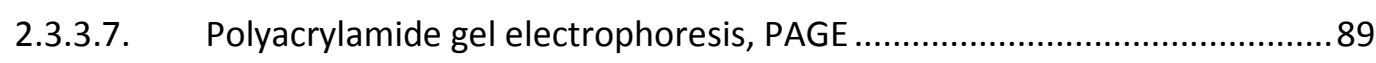

2.3.4. Results for the doubly modified system, s4.s5 ..............................................93

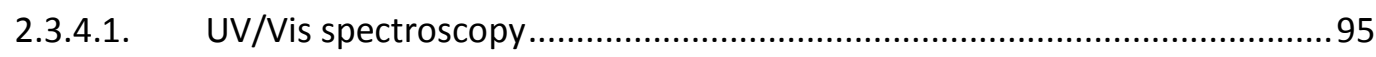

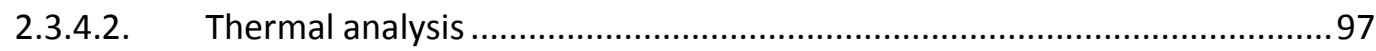

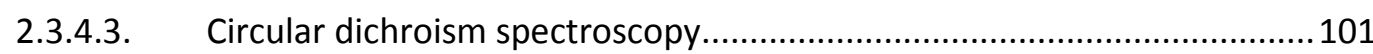

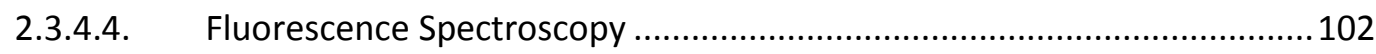

2.3.4.5. Polyacrylamide gel electrophoresis, PAGE ............................................. 102

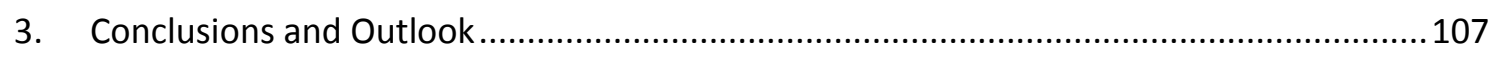

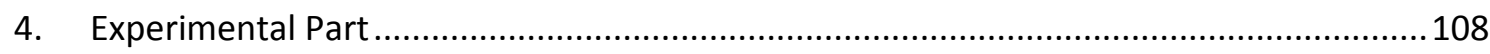

4.1. Syntheses of the ligands, complexes, nucleosides and phosphoramidites...............108

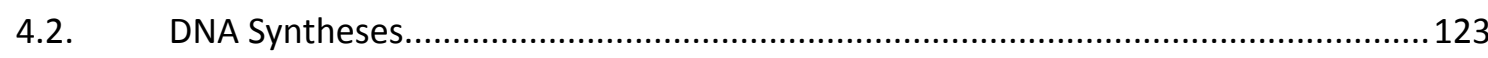

4.3. Purification by HPLC, detritylation and desalting ................................................ 125

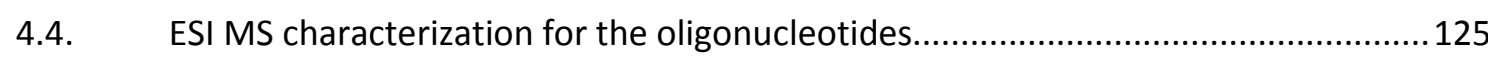

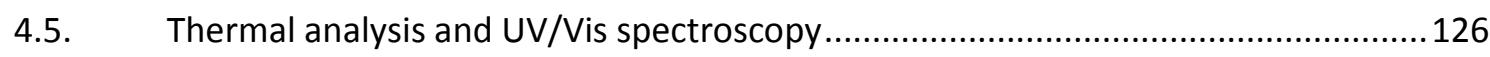

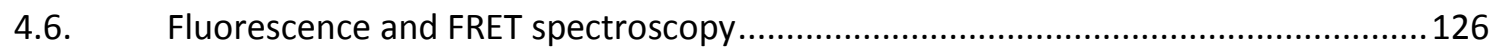

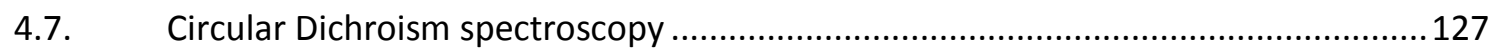


4.8. Polyacrylamide gel electrophoresis, PAGE

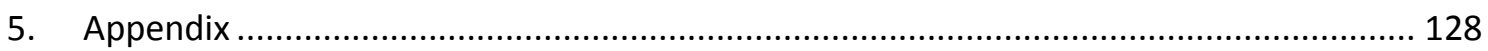

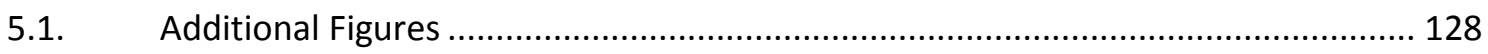

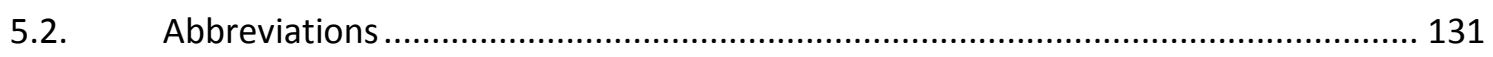

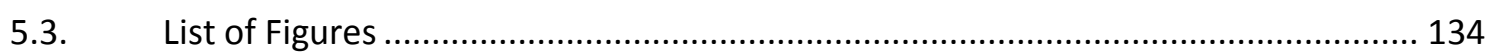

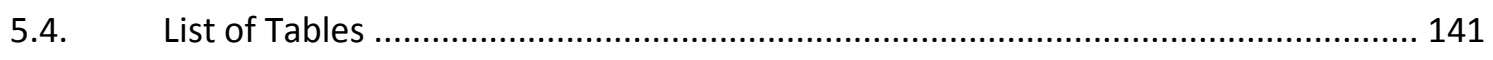

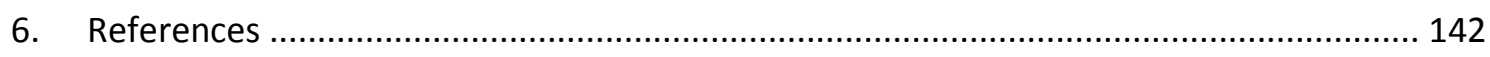





\section{Summary}

The work here presented explores DNA as a potential tool for supramolecular nanoconstructs, controlled self-assembly and the easy synthesis and manipulation of the DNA. Besides this, the introduction of chemical modifications to DNA is presented, which opens up possibilities of several applications such as charge transfer, nanomagnets, catalysis and many more. Important contributions were given and explored by the incorporation of artificial nucleobases and groups into DNA through the solid phase synthesis, where the formation of artificial metalbase pairing in recent years has brought several promising systems to the field with great stability. The concept of DNA branches and junctions based on the insertion of metalcomplexes expands the possibilities to produce structures such as DNA nanotubes and cages.

In the first part of this thesis it was described the syntheses and characterization of $\mathrm{Pd}$ (II) square-planar complexes with ligands based on a trans-chelating motif which produced selfassembled ring structures upon addition of bis-monodentate bridging ligands. Three or two membered rings were obtained as the product, depending upon the angle produced of the binding site of the bridging ligands added. The structures obtained provided information concerning thermodynamic and kinetic stability as well as further shed light on principles such as maximum site occupancy in supramolecular systems. The characterization of the building blocks and formation of the rings were carried out by NMR spectroscopy and ESI mass spectrometry. The controlled formation of metal complexes by introduction of the 1,2-bis(2pyridylethynyl)-benzene ligand into a library containing phenathroline and terpyridine ligands together with metal cationic species in a "one pot" system may be useful for future high ordered and metal controlled heteroleptic DNA systems.

The next part of this thesis explored the synthesis of ligands and artificial nucleobases as well as their incorporation into DNA. One of the approaches used also relied upon a trans-chelating building block suitable for solid phase DNA synthesis. This trans motif was internally incorporated into the DNA sequence to further produce square-planar metal-base pairing. The second approach relates to the synthesis of an artificial nucleoside designed to contain the trans-chelating motif pointing outside of the DNA duplex in a perpendicular direction tothe DNA helix to further, upon addition of $M(I I)$, allow the formation of interduplex cross-link through metal coordination. The characterization of the ligands was performed by NMR spectroscopy and ESI mass spectrometry, the latter of which was also used to characterize the synthesized DNA strands. Spectroscopic studies based on UV/Vis, thermal analysis, fluorescence and $C D$ spectroscopy were performed to study the formation and properties of the assembled DNA systems. Both unmetallated and metallated species were investigated and the stability of the metal-DNA constructs upon addition of $M(I I)$ was evaluated. Additional methods as FRET spectroscopy and ESI mass spectrometry were used to obtain more detail concerning the metal complexation observed, which involved the formation of monomeric or/and dimeric metal-DNA systems. Characterization of the metal-DNA systems and the interduplex cross-link were also pursued by polyacrylamide gel electrophoresis. 



\section{Introduction}

\subsection{Structure and function of Deoxyribonucleic acid (DNA)}

Deoxyribonucleic acid (DNA) is a biopolymer found in nature and responsible for the storage of genetic information. It consists of a chain based on sugar-phosphate backbone with a purine (adenine, $\mathrm{A}$, or guanine, $\mathrm{G}$ ) or pyrimidine (thymine, $\mathrm{T}$, or cytosine, $\mathrm{C}$ ) base linked to each sugar through the C1-carbon. Its molecular structure was elucidated by Watson and Crick ${ }^{[1]}$ when they presented the structural model for B-DNA. The B-DNA is the most frequently DNA secondary structure found in nature. There are two other major types of secondary structures, A-DNA and Z-DNA, these are less common than B form and have lower biological relevance.

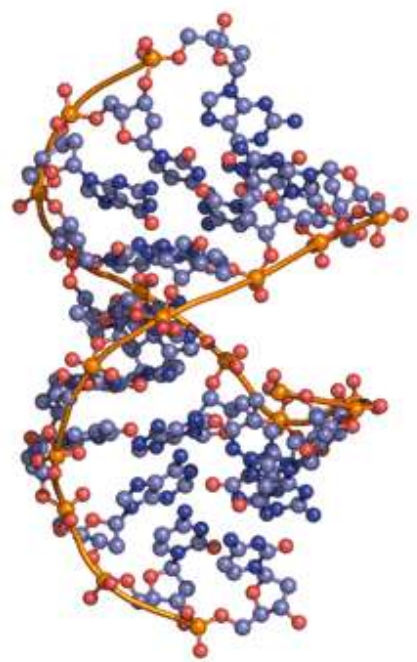

A-DNA

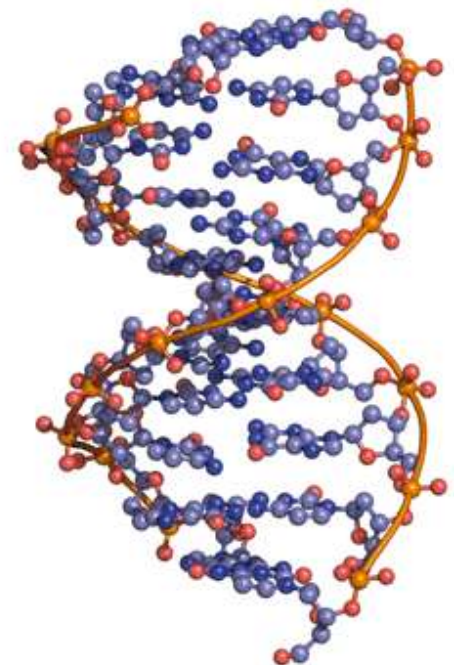

B-DNA

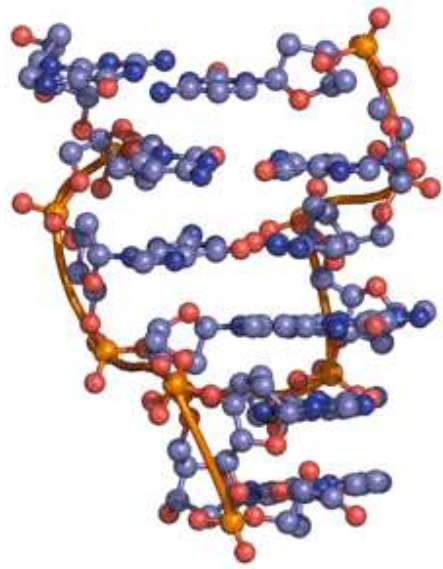

Z-DNA

Figure 1. Secondary structures for A-DNA, B-DNA and Z-DNA, respectively. ${ }^{[2]}$

These DNA secondary structures consist of a double helix formed through hydrogen bonding and $\pi$-stacking between two single complementary strands, wrapped around each other where each one is placed in an anti-parallel (reversed polarity) direction. The helix can be right-handed, as in B-DNA and A-DNA, or left-handed, as in Z-DNA. The deoxyribose sugars linked to the phosphate groups and bases can adopt different conformations; in high salt concentrations it is preferable a C3` endo conformation, characteristic for A-DNA, while B-DNA adopts $\mathrm{C}^{`}$ ' endo-conformation which provides a helix more elongated than the A form. Details about the conformations can be seen in Figure 2.

Regarding the double helix stability, the stacking interactions between the Watson-Crick bases are very important, $\pi$ stacking and dipole-dipole interactions from the neighboring bases, 
above and below, forming a hydrophobic centre. In B-DNA, the distance of the stacked bases is approximately $3.5 \AA$, which is the same as Van der Waals radius of planar aromatic compounds (the closest distance obtained before repulsive interactions start to occur) and the rotation between the adjacent neighbout base-pairs by $36^{\circ}$, where a complete turn occurs every $3.4 \mathrm{~nm}$ or about 10.5 base pairs. ${ }^{[3,4]}$
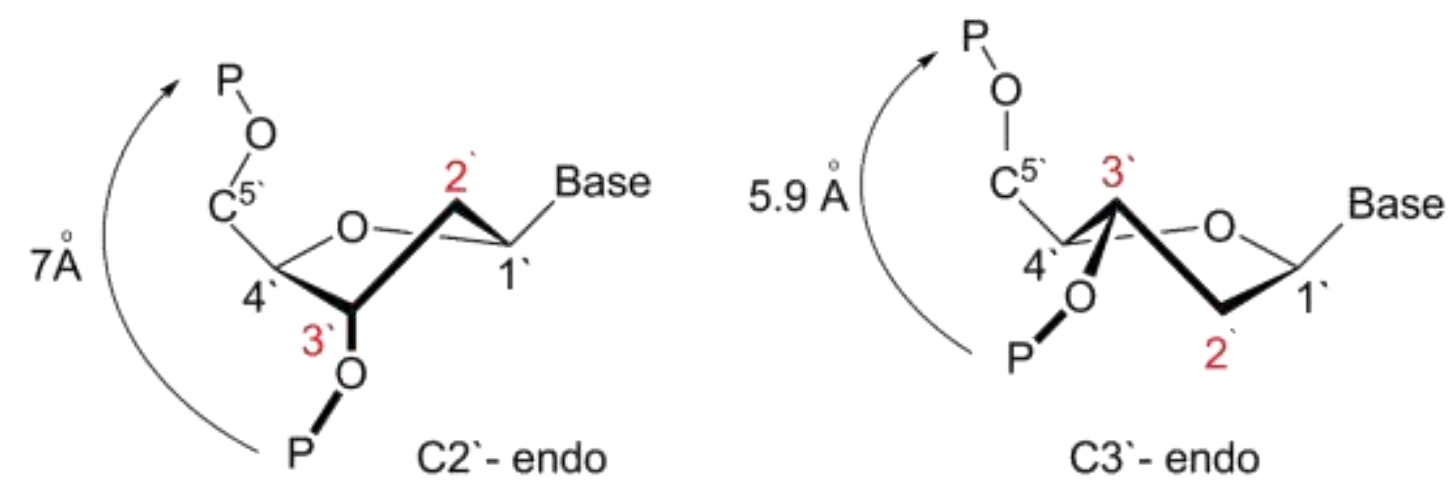

C3'- endo

Figure 2. Conformations for $C 2^{`}$-endo (B-DNA), where the $2^{`}$-carbon lies above the pyranose ring, and $C 3^{`}$-endo (A-DNA) where $3^{`}$-carbon is above the pyranose ring and the same side of $5^{\prime}$-carbon. ${ }^{[4,5]}$

The hydrogen bonds established between the base-pairs ( $A$ with $T$ and $C$ with $G$ ) between complementary single strands provides the double helix shape where the B-DNA shows two different types of grooves, denominated as major and minor grooves. The minor groove lies between $\mathrm{C}^{\prime}$ of the deoxyribose sugars on both strands while the major groove is on the opposite of the double helix. Recognition of major and minor grooves, sugar-phosphate binding, interactions between the stacked bases, together with new possibilities such as metalcoordination or covalent binding to the bases extend to a large number of possibilities in DNA application to supramolecular chemistry.

\subsection{Watson-Crick base pairs}

The Chargaff's rules estabilished that the base composition in DNA is $\chi_{A}=\chi_{T}$ and $\chi_{G}=\chi_{C}$, although it does not give information about the single strands. ${ }^{[6]} \mathrm{A}$ step further was given by $\mathrm{J}$. Watson and F. Crick, who proposed the idea of a double helix formed by complementary bases through hydrogen bonding interactions: $A$ with $T$ (or $U$ in case of RNA) and C with G.

There are two important features for Watson-Crick base pairs over other models (Hoogsteen and reversed types) which are characteristic of the B-DNA structure. The fact of being a ketoamine tautomeric form of the base pairs and the existence of a $C_{2}$-pseudo symmetry axis in the 
plane of the base pairs. This provides to all possibilities, A.T, T.A, G.C and C.G, the same environment in the double helix, without any distortion, as show on Figure 3 and 4.

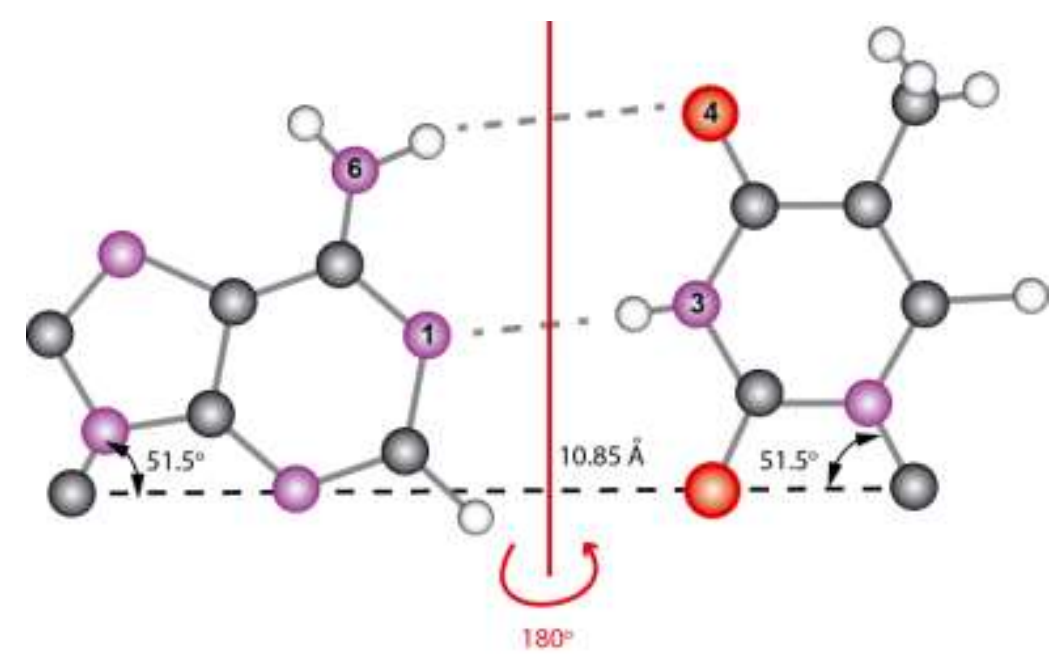

Figure 3. Watson-Crick base pair for A-T, two hydrogen bondings, N6 and 04 and N1 and N3.

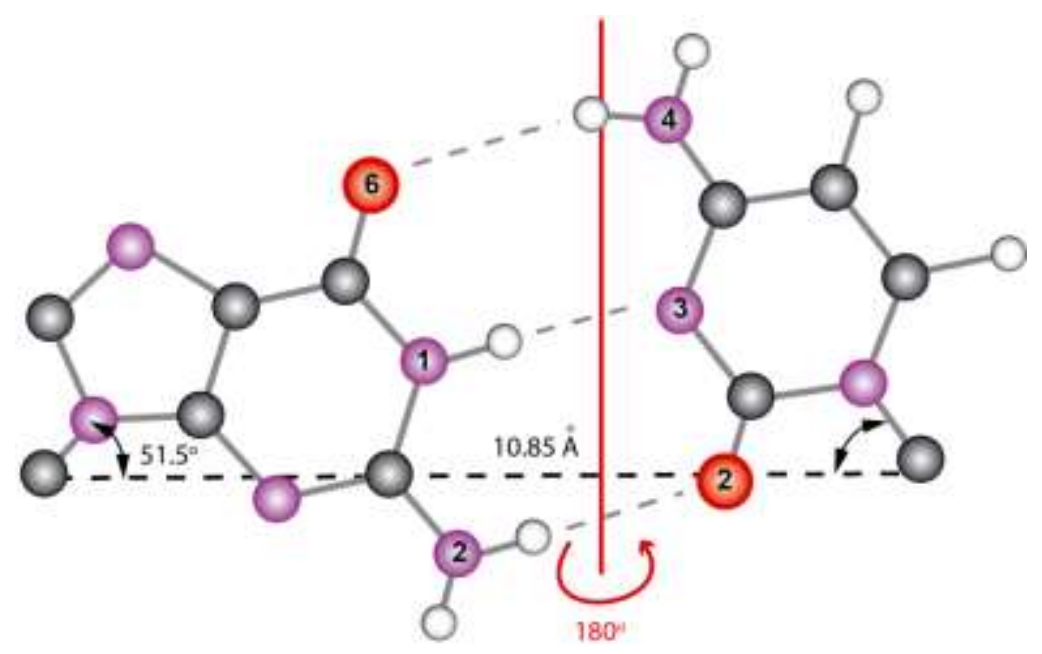

Figure 4. Watson-Crick base pair for G-C, three hydrogen bondings, $\mathrm{O} 6$ and N4, N1 and N3 and $\mathrm{N} 2$ and $\mathrm{O} 2$.

\subsection{Phosphodiester backbone}

Polynucleotides such as RNA and DNA contain, in their backbone, an alternating structure of sugars bound to phosphodiester groups. In RNA the sugar group is ribose and in DNA it is $2^{\circ}-$ deoxyribose. The sugar-phosphodiester backbone makes the oligonucleotides negatively charged, where the total charge is a sum of the single negative charges per phospodiester units in the chain, despite the ends of the strands where, for synthetic DNA, one strand has a free 5'-phosphate and the another complementary end a 3'-hydroxyl group. ${ }^{[4]}$ 


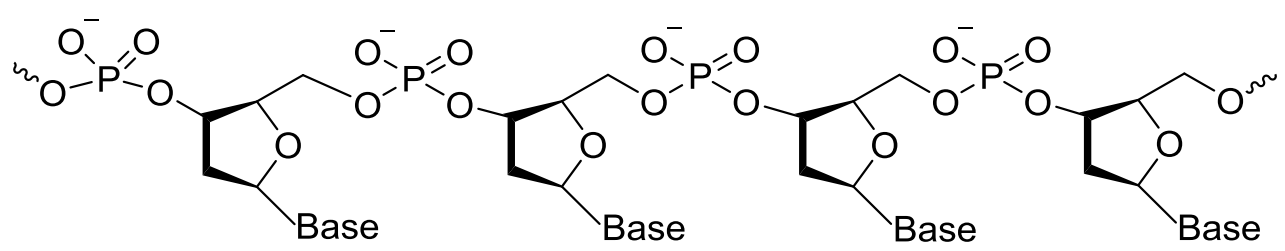

Figure 5. DNA phosphodiester backbone.

Ionic interactions between the negative charges of DNA and metal cations such as sodium, potassium and magnesium stabilize the DNA duplex, although, magnesium can perform a better stabilization due to the fact that it is a divalent cation and can interact with to two atoms of oxygen while the others mentioned, as monovalent species, bind to just one oxygen atom.

\subsection{Other DNA structures}

In nature, different types of structures can be observed for DNA, besides the duplex structure, such as triplexes and quadruplexes, Holliday junctions, supercoiled DNA and hairpins. ${ }^{[3,7]}$ Hairpin consists of a self-complementary single strand, where some bases (or even all) in the sequence match to each other forming Watson-Crick base pairing. Supercoiled DNA is when the B-DNA is over or under wrapped, it has an important role in biological processes for compacting DNA and packing it within cells, where the winding features are strongly dependent on $\mathrm{pH}$. Holliday junctions have an important role when chromosomes shift genes during the homologous recombination (the genetic process for exchange of the information or for DNA break repair based on nucleotides between two of the same DNA sequence or similar sequences in sister chromatids), it is a structure formed of four single DNA strands, complementary to each other in a way that enables them to form duplexes in a crossed shape. ${ }^{[5]}$ Once the junction is broken and splits, two linear duplexes can be obtained. It is also a promising approach for building blocks in DNA nanotechnology. ${ }^{[8,9]}$

Regions or sequences rich in purine or pyrimidine bases normally prefer structures not based on Watson-Crick base pairs, as for example quadruplexes, found in telomers and formed by Grich sequences through Hoogsteen base pairing. ${ }^{[3,10]}$ The carbonyl groups of each squareplanar array are pointing to the centre and can interact with monovalent cations such as $\mathrm{Na}^{+}$or $\mathrm{K}^{+}$. The quadruplex can be formed from a single long strand folded into itself three times or between two strands or even independent four strands, all having G-rich sequences. Hoogesten base pairing is also responsible for interactions between a duplex (major grooves of regions rich in $\mathrm{A}$ or $\mathrm{G}$ bases) with a third single strand, forming triplexes (Figure 6a). ${ }^{[1]}$ 
a)

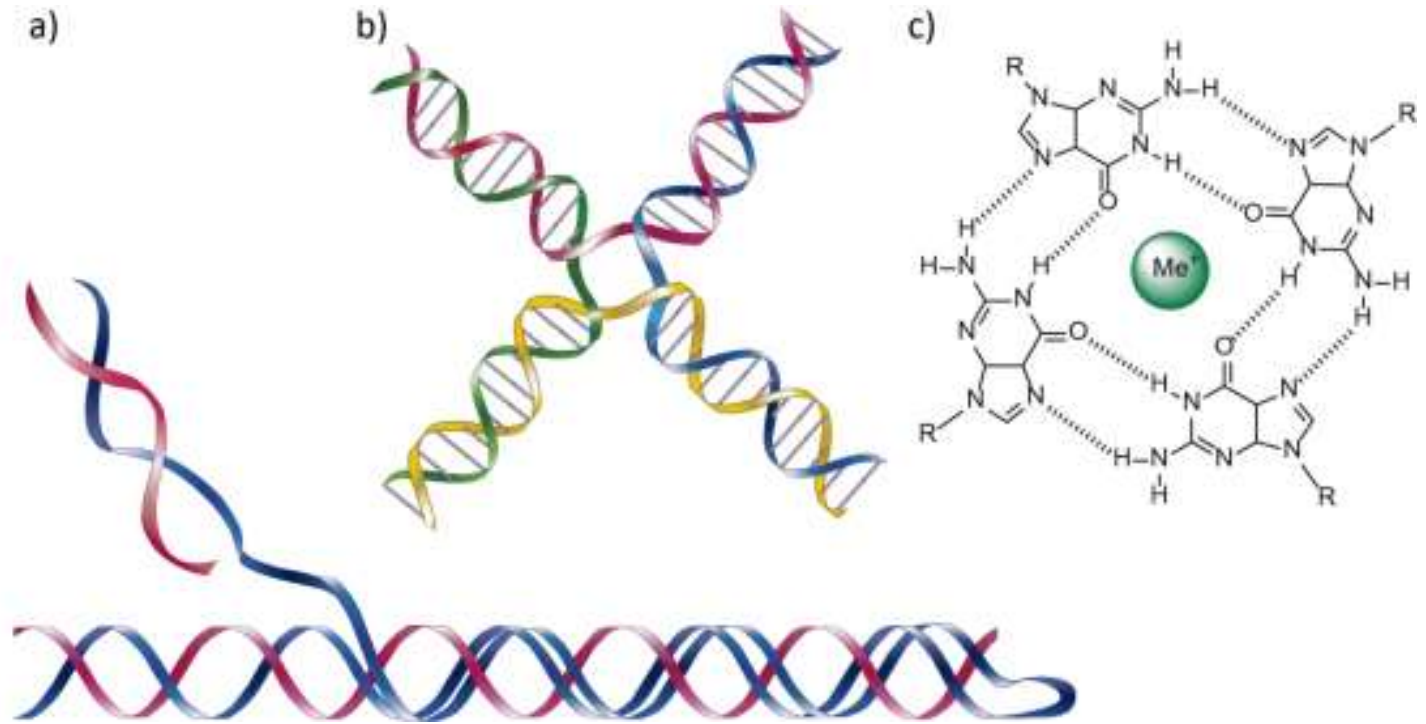

b) c)

Figure 6. a) DNA triplex, b) Holliday junction and c) Hoogsteen interaction in a G-quartet.

Failures in the DNA duplex can happen as for example from mismatched or damaged bases or single strand nicks. A common example occurs when neighbouring $\mathrm{T}$ bases at the same single strand, ss-DNA, under ultraviolet light react producing T dimers, which covalently cross-link the pyrimidine bases and causes distortion in the double helix. This case is the primary cause for melanoma in humans. ${ }^{[4,12]}$

Duplexes containing one of the single strands longer than the complementary, in a way to have at the both ends only single strand, can be used in DNA design for higher ordered structures, such approach is called "sticky ends". Other structures such as for example DNA catenanes, are possible to design, although they are not naturally observed. ${ }^{[13]}$ They can be synthesized and used in DNA nanotechnology, producing higher ordered structures. Seeman et al., pioneerred the design of 2D and 3D DNA structures, based on the self-assembly of complementary strands to plan the architecture of structures, ${ }^{[14]}$ been followed by Rothemund ${ }^{[15]}$ and the method called "DNA origami", a technique which makes use of a very long single DNA strand (in the mentioned work, genomic DNA from the virus M13mp18 as a scaffold) and several short complementary strands, called "staples", are designed to fold into itself due to the duplex formation regions providing $2 \mathrm{D}$ and $3 \mathrm{D}$ shapes such as smiley faces. Yan et al. also presented DNA origami with advanced curvatures for 3D DNA nanostructures. ${ }^{[16]}$ 
a

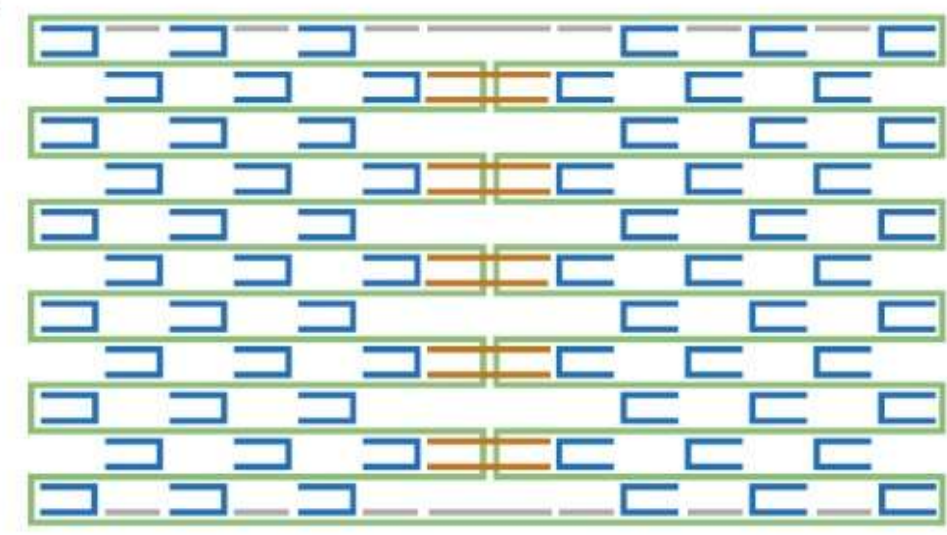

Template - Seam

Body

Edge b

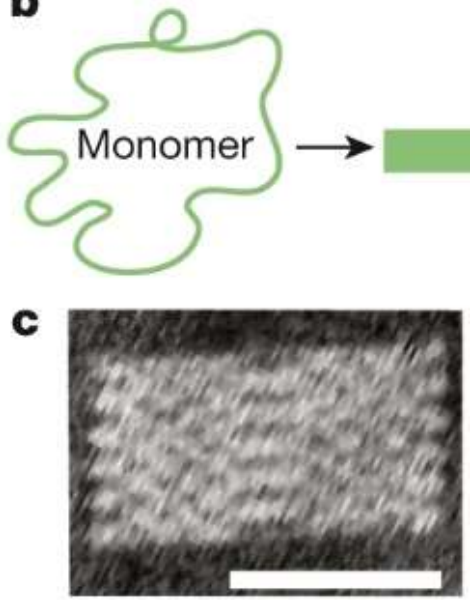

Figure 7. Scheme for a DNA Origami plan a) detailed plan for a monomeric retangular fold made with 66 body staples (blue) and edge staples (grey) hybridized into a template (green), b) Monomer template before and after annealed, c) Atomic force micrograph of respective monomer $(50 \mathrm{~nm}){ }^{[17]}$

\subsection{Metal coordination types in DNA}

\subsubsection{Metal coordination to unmodified DNA}

The interaction of metal cations with unmodified DNA can occur through two different methods. The first method is related to the direct binding of the metal to DNA through interaction with the base pairs. The second method involves the coordination of a metal complex with DNA, such as the cis-platinum compounds, or interactions between metal complexes with DNA through its grooves. ${ }^{[3,18]}$ Major and minor groove recognition has an important role and influence, been responsible for regulation of protein synthesis and zinc finger interactions with DNA, for example. Zinc finger proteins are involved in specific sequence binding to DNA and RNA, such interaction is related to their function or to perform a role in the transcriptional regulation process. The chemotherapeutic intervention through a metal-bind is an efficient approach that inhibits the function of the protein, well used in case of protozoal diseases with application of medicaments based on bismuth(III) and to inhibit the zinc finger CCHC type domain of human immunodeficiency virus type 1 (HIV-1) nucleocapsid 7, blocking the protein functions and as consequence the HIV-1 replication. ${ }^{[19]}$

As mentioned, hydrogen bonding between Watson-Crick bases are not the only interaction to be consider for duplex stability in DNA. Interactions between planar aromatic groups due to $\pi$ $\pi$ stacking of neighbouring bases also have an important role. The addition of aromatic planar 
groups into the minor or major grooves can be applied providing extra stability to ds-DNA and generates structural changes. ${ }^{[18,20]}$ The $\mathrm{Pt}(\mathrm{II})$ terpyridine complex developed by Lippard is a great example of the use of complexes as a smart way to provide, through the transition metal cation, a positive charge into the intercalator. ${ }^{[21]}$

The Barton group has several good examples of metal complexes acting as metallointercalators. $\mathrm{Rh}(\mathrm{III})$ complexes can be used as intercalators to recognize and react with DNA base mismatches, while Ru complexes are able to do additional features, such as DNA cleavage and luminescence. ${ }^{[22]}$ A pioneer work for charge transport, also from Barton et al., uses Rh and Ru complexes attached to opposite ends of a DNA duplex, where the Ru complex acts as an acceptor and the Ru complex is the photoexcited donor, been quenched by Ru complex over a distance of $4 \mathrm{~nm}$. ${ }^{[23]}$ The Tor group also made important studies concerning metal-ligand charge transfer (MLCT) of Ru complex as donor and Os complex as acceptor (complexes from $M(I I)$ binding with artificial nucleotides based on phenanthroline-deoxyuridine group), elucidating the distance dependence for charge transfer on DNA due to the distance between the Ru complex and Os. ${ }^{[24]}$
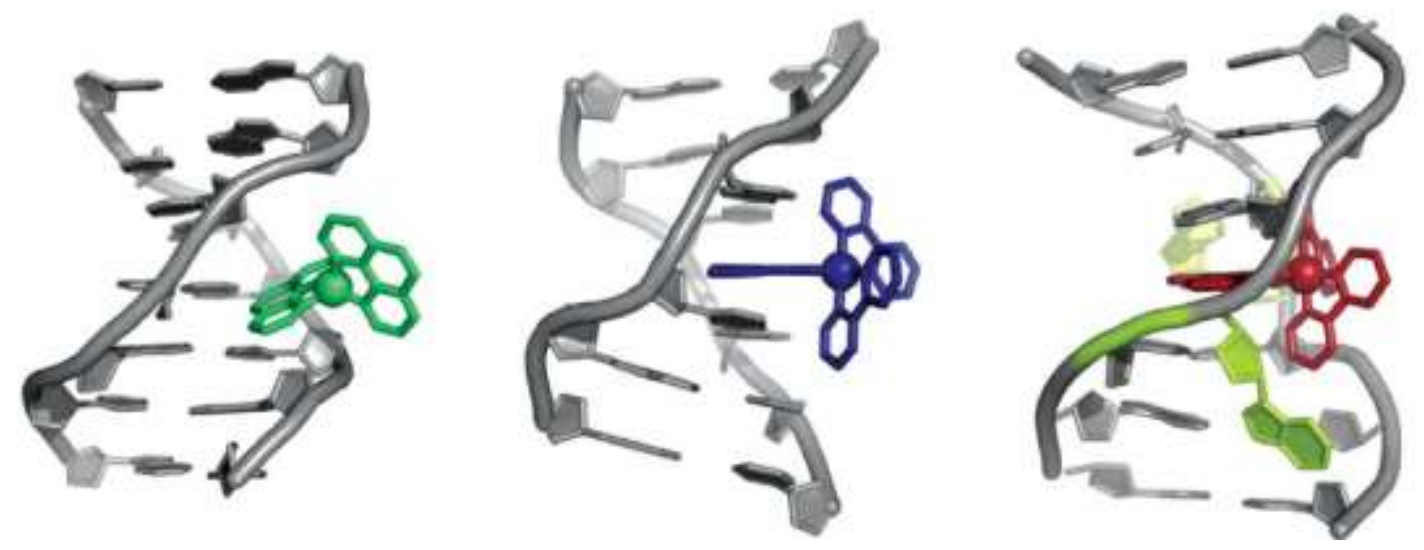

Figure 8. Three different modes of binding for complexes with $d s$-DNA, from left to right: groove binder, metallo-intercalator and metallo-insertor. Note that while metallo-intercalator interacts on major groove, metallo-insertor acts on minor groove, producing a stronger strain in the DNA due to replacement of the expelled base pairs after entering the DNA double helix. ${ }^{[22]}$

For octahedral complexes, the structure allows metallo-interaction between co-ligands with the neighbour bases, above and below, the one which is interacting with the planar aromatic group (Figure 9). It is from the Barton group the first example of metallo-intercalator DNA which was able to be characterized by X-ray crystallography (with $1,2 \AA$ resolution crystal structure). ${ }^{[25]}$ 


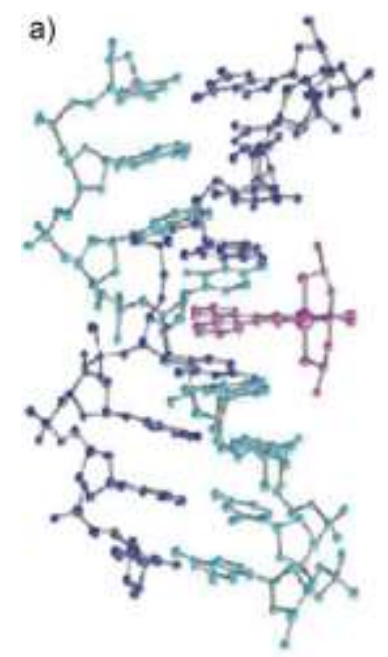

b)

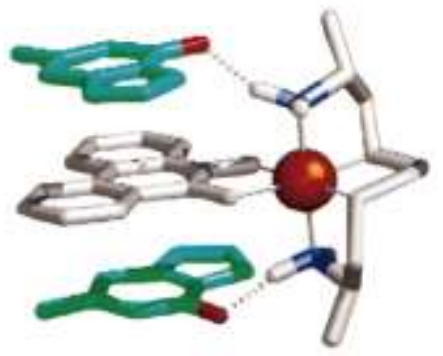

Figure 9. a) Selective intercalation of $\left[\mathrm{Rh}(\mathrm{phi})\left(\mathrm{Me}_{2} \text { trien) }\right]^{3+}\right.$ into the major groove of the $d s$ DNA, 5'-TG/CA-3'. Phi= 9,10 -phenanthrenequinone diamine and $\mathrm{Me}_{2}$ trien= 2R,9R-diamino4,7-diazodecane. b) Specific hydrogen bonds between the intercalator and the oxygen atoms O6 of the guanines on the intercalation site, $3.0 \AA{ }^{[25]}$

A case of intrastrand cross-link without metal interaction happens between neighbour $\mathrm{T}$ bases forming T-dimers being covalently linked by photoreaction. ${ }^{[5]}$ Although, the most known and widespread examples of intra- and interstrand cross-links are adducts formed between DNA and cis-platinum complexes. The approach provides to a square-planar Pt(II) complex such as $\left[\mathrm{Pt}\left(\mathrm{NH}_{2}\right)_{2} \mathrm{Cl}_{2}\right]$ and the ability to bind to DNA through the N7 of purines, preferable in GG or cis-AG adjacent bases, providing 1,2-intrastrand cross-links. ${ }^{[18]}$
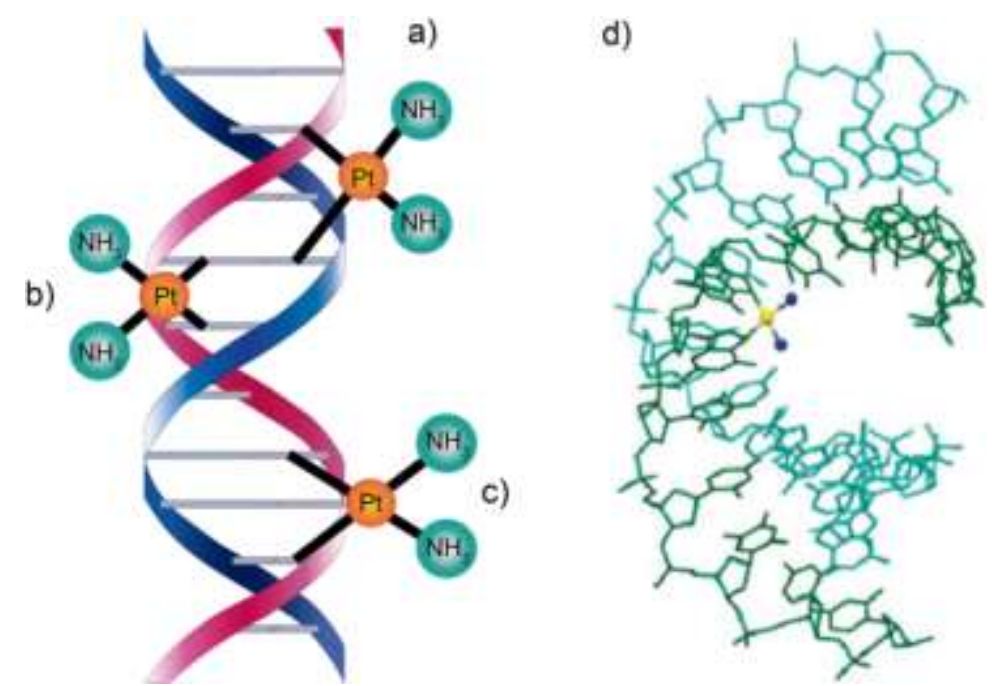

Figure 10. Types of cross-links between cis-platinum and ds-DNA a) interstrand cross-link b) 1,2-intrastrand cross-link, c) 1,3-intrastrand cross-link, d) scheme of a kink caused in the dsDNA due to the coordination of cis-platinum with $\mathrm{G}$ nucleotides on the DNA. ${ }^{[3]}$ 
The adduct formation causes a kink on the DNA duplex in the major groove, it unwinds the DNA and provides a loss of stability, interfering with function and development for replication steps, for example. ${ }^{[26]}$ The formation of 1,3-intrastrand cross-links between non-adjacent guanines are also possible (Figure 10), such as interstrand cross-links between bases of complementary strands. The latter occurs less often, but also through the N7 atom of purine bases with platinum. ${ }^{[27]}$

\subsubsection{Metal coordination in modified DNA}

The synthesis of modified DNA involves the insertion of artificial chemical groups into the oligonucleotides and through this, the incorporation of new properties into DNA, which expands possibilities for DNA to be used as material for nanodevice design. The addition of groups that can act as ligands and are able to form metal complexes can transfer to the DNA structural features, through the complex geometry, properties such as enhanced stability, metallic binding selectivity and controlled switching. DNA modifications can follow two approaches: it happens through the conjugation of ligands (complex formation upon addition of metal) to the ends of the DNA helix or by the incorporation of artificial nucleosides by solid phase synthesis.

The artificial nucleosides are synthesized and converted to phosphoramidites before applied in solid phase synthesis. They can keep the structure of DNA nucleotides (based on sugarphosphate backbone) containing an artificial base which differs from A, T, C and G. Due to its lower cost and effectiviness the 5-iodo- 2 -deoxyuridine, dIU, is frequently used as starting material, where the functional group planned to be used can be attached via Sonogashira cross-coupling containing an alkyne or propargylamide group as linkers. ${ }^{[9,28,29]}$

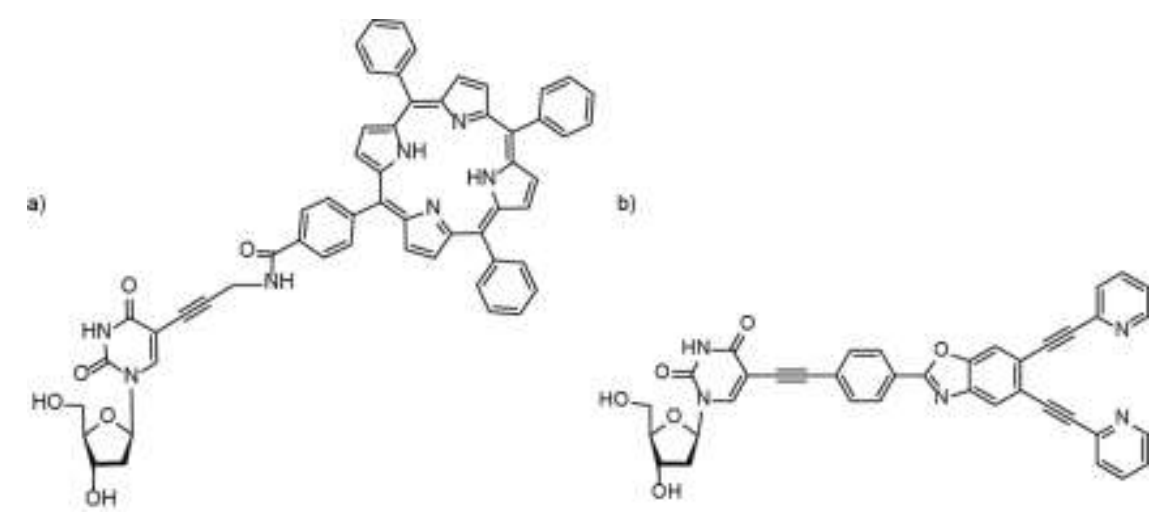

Figure 11. Examples of artificial nucleosides based on 5-iodo-2 -deoxyuridine, dIU. a) phorphyrin group attached through 5 -propargylamine linker to 2 -deoxyuridine. ${ }^{[29]}$ b) trans- 
chelating oxazol group attached through 5 -alkyne linker to 2 -deoxyuridine. ${ }^{[30]}$ For solid phase DNA synthesis further steps as DMT protection at the 5'-position and phosphoramidation at $3{ }^{`}$-position are required. More synthetic details are given in the experimental part.

This approach is explored for external modifications to the DNA duplex and were already applied to synthesized modified DNA containing nucleotides based on terpyridine, bipyridine and phenanthroline groups. ${ }^{[31,32,33]}$ Modified DNA strands containing artificial nucleotide based on porphyrin are also well explored for several applications. The Stulz group developed for electron transfer efficiency studies a system based on porphyrin zipper arrays containing up to 12 porphyrins. ${ }^{[29]}$ Porphyrin nucleotides are also been applied for multibranched DNA nanoarchitectures. ${ }^{[34]}$ Howorka et al. developed DNA nanostructures tagged with porphyrins as a strategy to enlarge and enrich membrane-spanning nanopores. ${ }^{[35]}$ The only requirement basically necessary to incorporate a building block into DNA is a hydroxy group, which will be phosphitylated and, once converted to phosphoramidite, can be applied for solid phase DNA synthesis. ${ }^{[9,36]}$
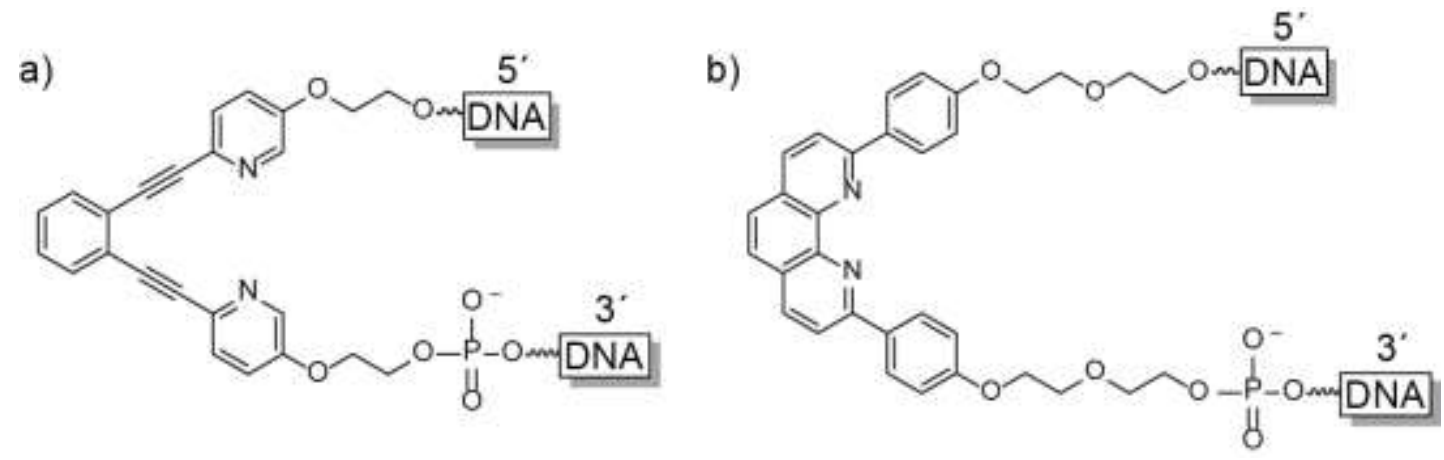

Figure 12. Examples of ligands incorporated into DNA a) trans-chelating type, ${ }^{[37]}$ b) phenanthroline type. ${ }^{[38,39]}$ Building block required for SPS containing $5{ }^{`}$-DMT protected and $3{ }^{`}$ phosphoramidation.

This approach was used by Sleiman et al. to develop metal-DNA branched junctions. Building blocks based on diphenyl-phenanthroline and terpyridine substituted by ethylene glycol group were incorporated into DNA and once the duplex is formed, its chirality is transfered to the ligands and further to the complexes. ${ }^{[39]}$ Both ligands were also combined giving heteroleptic metal-DNA assemblies (Figure 13), involving Fe(II)tpy $2: a b, C u(I) d p p_{2}: a b$ and $\mathrm{Cu}(I I) t p y: d p p: a b$ (the letters $\mathrm{a}$ and $\mathrm{b}$ are applied as nomenclature for complementary DNA single strands to facilitate the interpretation of which strand contains each ligand, in case of a heteroleptic system). Based on this structure, it was possible to develope nanotubular structures. ${ }^{[0]}$ Nevertheless, despite the possibility of binding different types of metal cations, it was not explored possibilities to produce multimetallic systems, been obtained at first time on metal- 
base pairing by Carrel group and presented in more details further at section 1.5.4. ${ }^{[41]}$ Häner et al., synthesized DNA strands containing at the terminal positions phenanthroline and bipyridine groups, placed near each other once the complementary strands hybridizes and after $\mathrm{Cu}$ addition it forms 3 possible types of complexes based on the $\mathrm{Cu}(\mathrm{I}) / \mathrm{Cu}(\mathrm{II})$ states, $\mathrm{Cu}(\mathrm{bpy})_{2}, \mathrm{Cu}(\text { phen })_{2}$ or $\mathrm{Cu}\left(\right.$ phen)(bpy). ${ }^{[42]}$

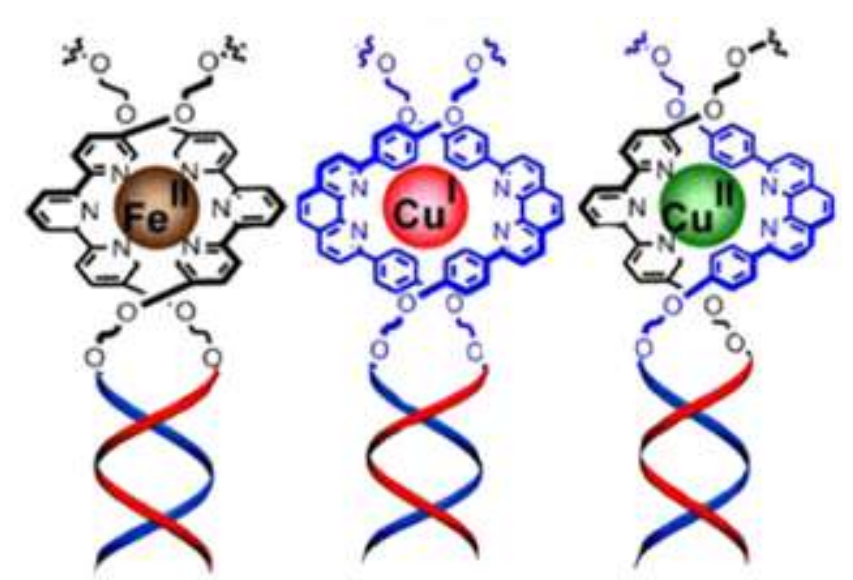

Figure 13. Scheme for modified DNA containing metal complexation between $\mathrm{Fe}(\mathrm{II}), \mathrm{Cu}(\mathrm{I})$ and $\mathrm{Cu}(\mathrm{II})$ with groups based on terpyridine and bipyridine in homoleptic and heteroleptic modified DNA duplexes. ${ }^{[39]}$

Amino, carboxy, and thiol groups can be used as modifiers in phosphoramidites to attach ligands or complexes to the $3^{`}$ - or $5{ }^{`}$ - terminus of DNA single strands. ${ }^{[43,44]}$ Aldehydes, acetylenes and azides (post-synthetically by click-chemistry) have recently also been applied. ${ }^{[45]}$ Barton et al., tethered covalently a Ru-complex to the 5 - end of a $15 \mathrm{mer}$ functionalized with a hexamethylene amine. The complex acts as metallointercalator and was used for photoinduced electron transfer on the helix interacting with the major groove once the duplex is formed. ${ }^{[43]}$ Krämer et al. attached terpyridine groups to amino functions at the $3{ }^{`}$ - and 5 - terminus of a single strand to form a DNA cyclic structure (Figure 14) upon metal coordination ${ }^{[44]}$, where two terpyridine groups attached to the ends of the ss-DNA strand are cyclic connected by addition of Zn(II) competing with duplex formation. The excess of Zn(II) causes opening of the cycle (through monomeric complexes formation) and it allows again the possibility of duplex hybridization. 


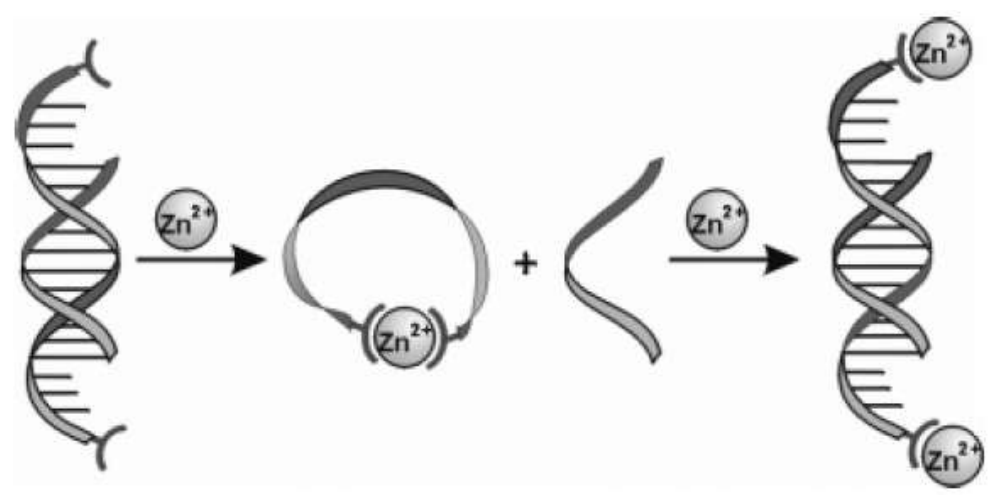

Figure 14. Formation of cyclic [Zn(II).ss-DNA] structure. ${ }^{[44]}$

Sleiman et al. made Ru-bipyridine complexes (bis-phosphoramidite) attached to two single DNA strands (5-terminus DMT protected), in a symmetric way that produces DNA arms, which are self-assembled to another arm of Ru-complex DNA, forming then a cyclic structure driven by the Ru-complexes orientation. ${ }^{[46]}$ There are cases where the ligand is attached at the 2 position of a nucleoside, for example, the three-way junction developed by Shionoya et al., where this position was found crucial to drive the ligands to the centre of the junction core. This kind of structure gives new possibilities of DNA edges and corners in higher ordered structures. ${ }^{[47]}$

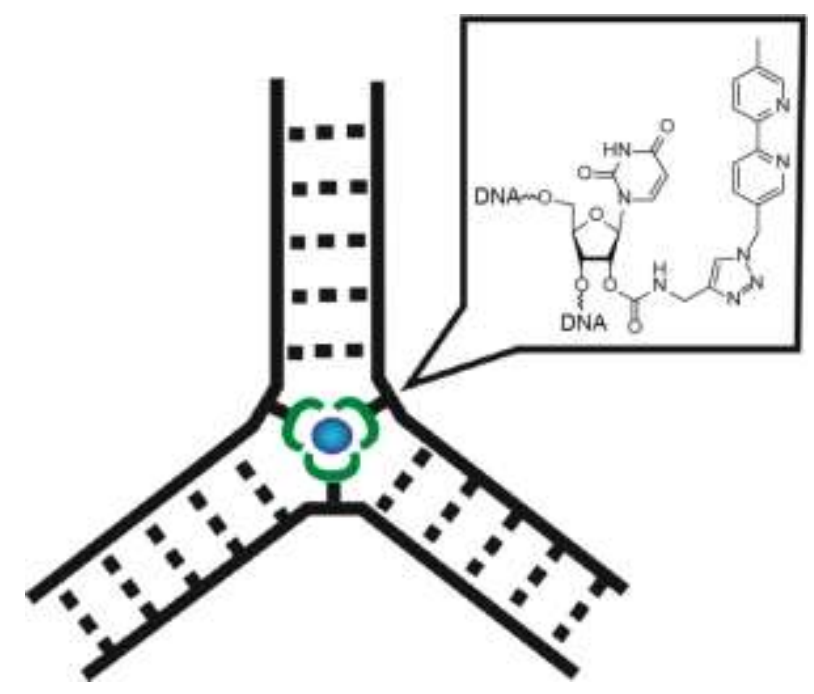

Figure 15 . Schematic detail for the DNA 3-way junction developed by Shionoya group. ${ }^{[4]}$

\subsubsection{Metal-base pairing}

Metal-base pairs(MBP), artifcial or not, are not based on hydrogen bonds between two bases as it happens in Watson-Crick base pairing. Instead of it, two bases or ligands incorporated into DNA strands (and placed to face each other under ds-DNA formation) are mediated by metal complexation. Evidence by NMR of metal-base pair was provided by Buncel et. al. ${ }^{[48]}$, for T-T mismatches in the presence of $\mathrm{Hg}(\mathrm{II})$, proposed before by Katz in 1963. ${ }^{[49]}$ 


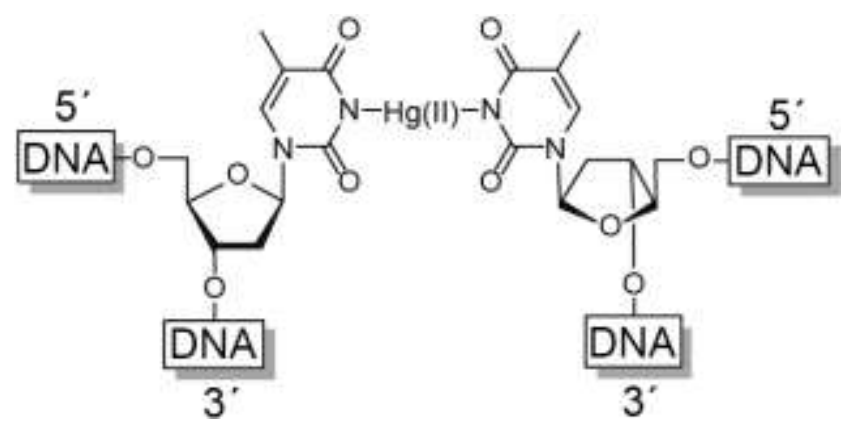

Figure 16. Metal-base pairing between T-T mismatch and $\mathrm{Hg}(\mathrm{II})$ proposed by Katz. ${ }^{[49]}$

The design of ligands for artificial metal-base pairing must be well planned to avoid steric hindrance and disturb the helix structure. For successful systems it should take into account the flexibility of the ligand, presence of aromatic groups for $\pi$ stacking with neighbouring bases, the metal species applied for complexation and geometry of the complex formed in agreement with the helical shape of DNA in orded to not cause distortions and instability to the duplex. The DNA stability can be studied by thermal analysis, where one of the greatest feature in systems containing MBP is the extra stability given to the DNA duplex and which can be observed due to an increase of the DNA melting point, which is the temperature when annealed strands and melted duplex are presented in equal amounts. ${ }^{[50]}$ Such systems can be applied for nanomagnets, nanowires and charge transfer. ${ }^{[8,51]}$

The first artificial metal-base pairing was developed by Shionoya et al. ${ }^{[52]}$ It was designed as an independent unit, not been incorporated into DNA and consists of a square-planar complex between $\mathrm{Pd}(\mathrm{II})$ and amine groups as binding sites on the ligands. Schultz group provided a $\mathrm{CU}(\mathrm{II})$-base pair incorporated into DNA, which could be characterized by X-ray analysis and presented the Z-DNA conformation, also evidenced by CD spectroscopy, even under low salt concentrations. ${ }^{[53]}$ Meggers et al., designed a metal-base pairinng with a C3 backbone and a ligand with a hydrophobic aromatic surface exhibiting strong binding to $\mathrm{Cu}(\mathrm{II})$. The result is an increase of more than $20^{\circ} \mathrm{C}$ on the melting point (compared to the unmodified DNA having an A-T as base pair). ${ }^{[54]}$ 
(a)

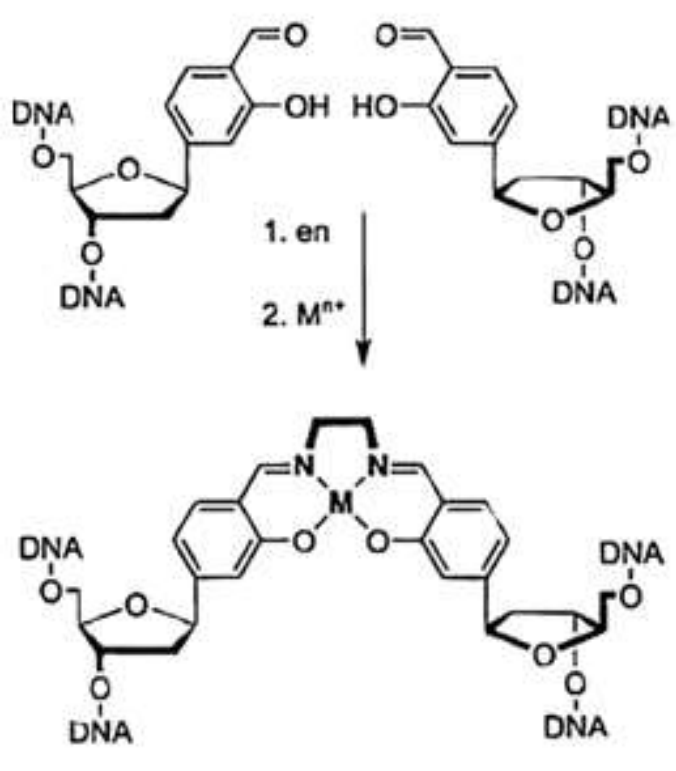

(b)
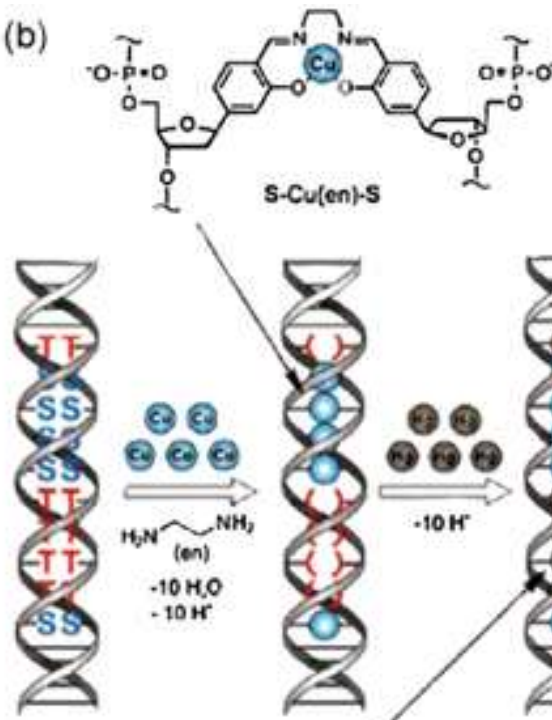
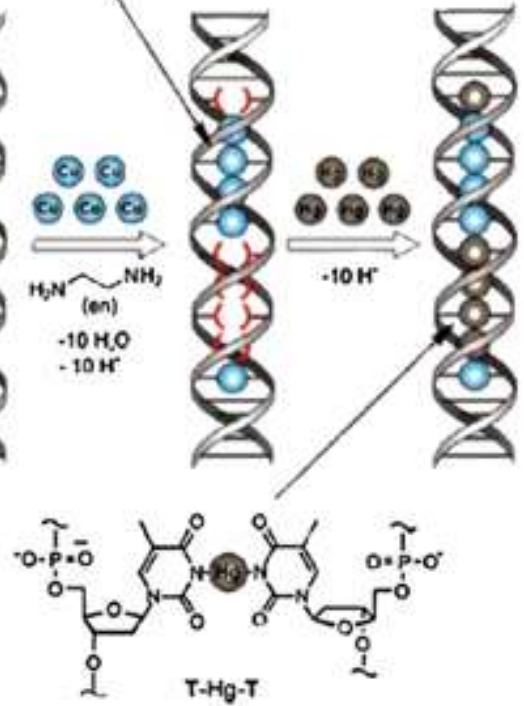

Figure 17. a) Scheme for the salen-base pairing complex, b) DNA duplex in a stack with multiple $\mathrm{Cu}(\mathrm{II})$ and $\mathrm{Hg}(\mathrm{II})$ complexes. ${ }^{[55]}$

A DNA duplex containing up to five $\mathrm{Cu}(\mathrm{II})$ complexes was obtained by Shionoya group for a system based on hydroxypyridone. ${ }^{[56]}$ This was followed by the Carell group, that developed a metal-base pairing based on salen group ( $\mathrm{N}, \mathrm{N}^{\prime}$-ethylene-bis(salicylimine)) and linked by ethylene-diamine besides the $\mathrm{Cu}(\mathrm{II})$ or $\mathrm{Mn}$ (III) coordination. ${ }^{[55]}$ As a result, it was obtained the strongest duplex stabilization so far observed for a DNA duplex and metal-base pairing. This design demonstrated ability to have up to 10 metal-base pairs stacked in a row. ${ }^{[41]}$ Furthermore, Carell and Shionoya reported a modified DNA containing two different systems to form controlled metal-base pairs, where they combined $\mathrm{Hg}$ (II) binding to T-T mismatches and $\mathrm{Cu}(\mathrm{II})$ ions to salen ligands incorporated into the DNA, providing metal-base pairing of both systems. Until the current days, this is the only example of a programmed multiple metalbase pairing system. ${ }^{[57]}$

\subsubsection{Inter cross-link between DNA duplexes}

Attempts to link DNA duplexes through complex formation between artificial nucleotides upon addition of metal were developed as a new potential approach to develop higher ordered DNA nanostructures. Currently, most of all the attempts for such systems were based on nucleotides containing terpyridine groups. The design for such structures established that such modifications should contain the group part responsible for metal coordination pointing 
outside of the DNA duplexes. The synthesis of these building blocks attached the terpyridine groups to 5-iodo-2'deoxyuridine via Sonogashira cross-coupling. Wagenknecht et al., developed a short terpy building block, connecting the terpyridine group containing only an alkyne linker binding the uridine base. ${ }^{[58]}$ Evidences of monomeric complexes between DNA$\mathrm{M}$ (II) for $\mathrm{Fe}(\mathrm{II})$ and $\mathrm{Ni}(\mathrm{II})$ could be observed, although not strong evidences to conclude "networking between DNA duplexes".

a)

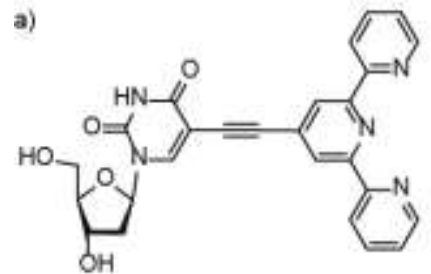

b)

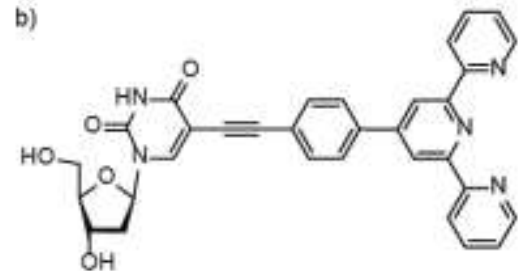

c)

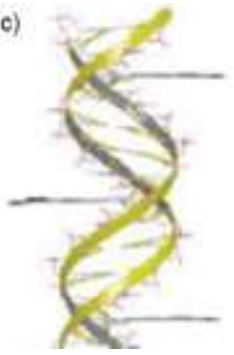

Figure 18. Deprotected nucleosides based on tepy into dIU a) Wagenknecht approach, ${ }^{[58]}$ b) Stulz approach, ${ }^{[30]}$ c) illustrative idea how is the group on b) pointing outside of the $d s$-DNA.

The Stulz group has earlier also tried similar approach, using a longer terpy building block, with a benzo-alkyne linker. In this approach the complementary strand is longer, producing DNA duplexes with one single modification and containing sticky ends, which are able to assemble to other units of such duplexes and produce longer ds-DNA which will contain multiple nucleotides with outside pointing terpyridine groups in both sides of the duplex. This approach expected to produce 2D grids once the terpy modifications form complexes with Fe(II) addition. The evidences observed according to transmission electron microscopy (TEM) images suggest the formation of nanotubes structures. ${ }^{[30]}$ The last example comes from Hocek group, which tried to make such interstrand link between duplexes with building blocks based in terpyridine and also bipyridine groups connected via octadiyne linker. ${ }^{\text {[2] }}$ A single modified strand containing two building blocks showed formation of an interstrand complex on the same ds-DNA, due to $M(I I)$ addition (Figure 19b). No formation of intercross link through complexation between the building blocks from different $d s$-DNA could be observed. 

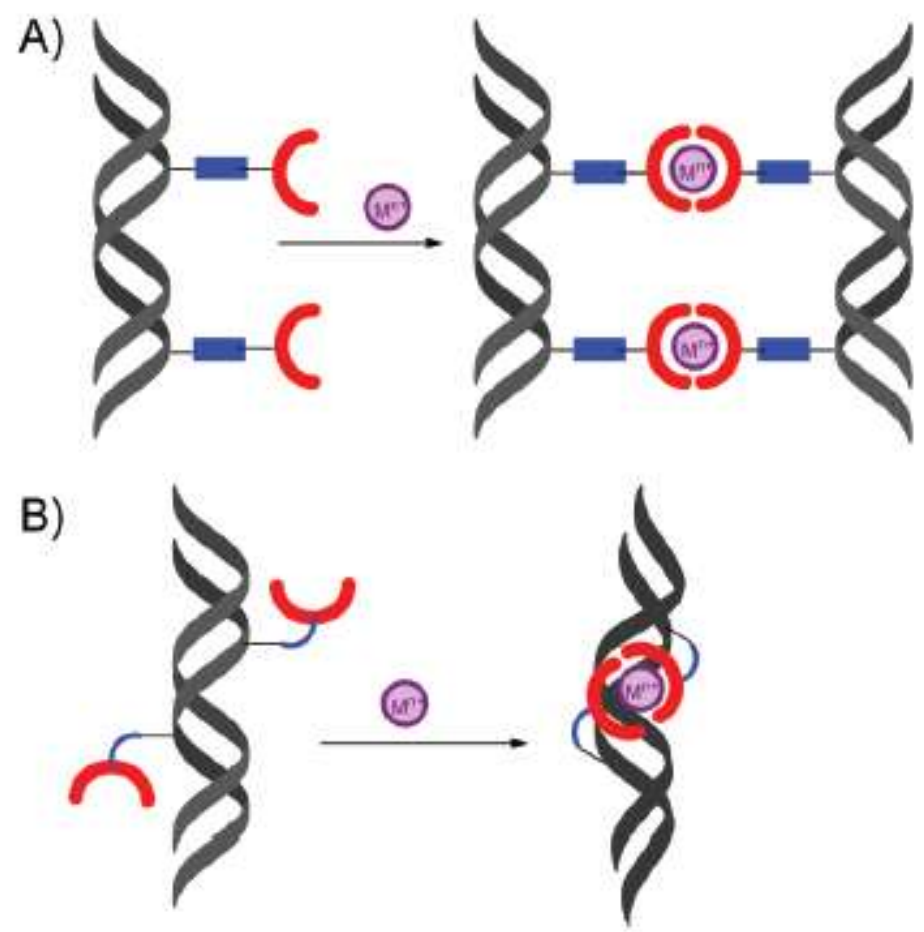

Figure 19. Representation of a) dimer complex formed by interduplex cross-link between DNA due to metal coordination and b) dimeric complex between two modifications on the same $d s$ DNA. ${ }^{[32]}$

\subsection{Theory for synthetic, analytic and spectroscopic methods}

\subsubsection{Solid Phase DNA Synthesis}

Solid phase DNA synthesis is an automated process that works step by step, coupling base by base according to a cyclic process as can be observed in the scheme below. ${ }^{[59]}$ Despite the advantage of an automated process, it is able to incorporate into oligonucleotides artificial bases at specific positions previously planned, expanding the nucleobase alphabet and potential of DNA as a tool for nanoconstructs and supramolecular chemistry applications. It is also a fast and simple synthetic method with washing processes between all the steps, where impurities are washed away thus saving time on further purifications.

The synthesis process occurs from $3^{`}$-to $55^{`}$ - direction along the phosphodiester backbone, in contrast to biosynthetic DNA replication. The process begins with a $3^{`}$-nucleoside as first base, A, C, T or G, according to the oligonucleotide sequence desired to be synthesized. The first nucleobase in a ss-DNA sequence is attached to a solid support (insoluble resin which is normally controlled pore glass (CPG) or polystyrene and inside of a plastic column 50-200 $\mu \mathrm{m}$ 
diameter) that allows solvents and reagents for each synthetic step pass through, while under inert atmospherice.

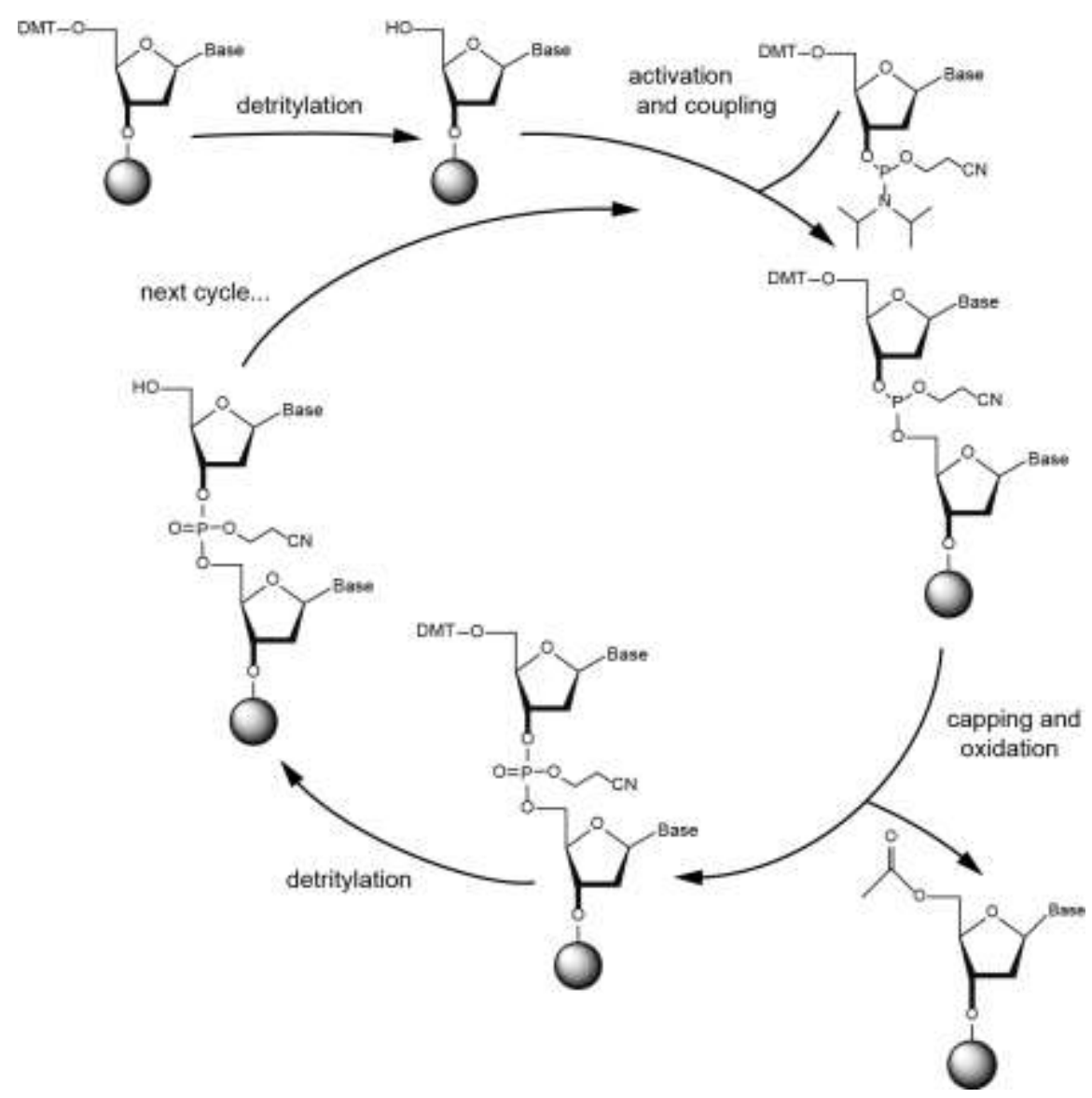

Figure 20. Scheme outlining the process of solid phase DNA synthesis.

The first step of the coupling process is detritylation. The 3 -nucleoside contains a $4-4$ 'dimethoxytrityl, DMT, protecting group at the $5{ }^{`}$-position. This protection is present in all the further building blocks that will be added, as it is necessary to avoid polymerization during the solid support functionalization, such as base induced $5{ }^{`}-\mathrm{OH}$ nucleophilic attack. The DMT group has to be removed to allow the next base to be bound during the further processes on the reactional cycle. Cleavage of DMT is commonly achieved using a $3 \%$ trichloro acetic acid solution (TCA) in dichloro-methane, giving an orange DMT cation solution. The DMT cation absorbs in the region at $495 \mathrm{~nm}$ and the cleavage process can be followed by UV/Vis and its intensity used as an indirect way to measure and monitor base coupling efficiency.

Prior to the next step, activation and coupling, the column is washed with dry acetonitrile. All the following bases are added as a building block based on phosphoramidite monomers (standard 0.1 M solutions in dry acetonitrile) which contains $5{ }^{`}$-DMT protected and at 3 `position, bound to the reactive $\mathrm{P}(\mathrm{III})$, a $\beta$-cyanoethyl and diisopropylamine as protecting 
groups. The activation resumes to protonate the 3 -diisopropylamine group of the next programmed phosphoramidite to be attached. The activator is based on tetrazole derivatives, as weak organic acids (pKa 4.6) but enough to protonate the diisopropylamine group although not strong enough to cause an undesired further DMT deprotection. Once the protecting group diisopropylamine is protonated, it becomes a good leaving group and the position is susceptible to be attacked by $5{ }^{-}-\mathrm{OH}$ from the nucleoside attached to the solid support by $\mathrm{S}_{\mathrm{N}} 2$ reaction giving a trivalent phosphite triester. The coupling step can be programed to be longer than the other steps to improve the coupling efficiency and oligonucleotide yield.

The next step after coupling is highly important and denominated as "capping". The capping step ensures that $5^{-} \mathrm{-OH}$ unreacted is whashed away to avoid it to bind in the next coupling step with the following phosphoramidite, producing failure strands. On this step, there are two solutions, one is $\mathrm{N}$-methylimidazole, Melm, in a mixture of dry tetrahydrofurane, THF, and pyridine (Cap A) and the another solution (Cap B) contains acetic anhydride in pyridine/THF. The mixture of solutions gives acetylation of the $5^{\prime}-\mathrm{OH}$ groups, making them inert to next steps. The pyridine makes sure the $\mathrm{pH}$ stays basic to not cause detritylation when the acetic acid is formed from the acetic anhydride reaction with methyl-imidazole.

The fourth step is an oxidation where the phosphite triester $P(I I I)$ formed is converted to $P(V)$ before the next detritylation. This is important because the $\mathrm{P}(\mathrm{III})$ compound is unstable under the acidic conditions for DMT deprotection. The oxidation is performed by iodine oxidation with water and pyridine. The $\mathrm{P}(\mathrm{III})$ becomes protonated after reacts with $\mathrm{I}_{2}$ becoming susceptible to a nucleophilic attack by $\mathrm{H}_{2} \mathrm{O}$, releasing then pyridinium iodide. Further pyridine attack in hydroxyl group bound to $\mathrm{P}(\mathrm{III})$ provides the final conversion to $\mathrm{P}(\mathrm{V})$ through formation of the phosphotriester protected by a $\beta$-cyanoethyl group.

A second capping step is necessary, this time to get rid of residual water from the oxidation process. The water can reduce the efficiency in the next coupling. The acylating agent reacts with residual water giving acetic acid, which is also washed through by dry THF/pyridine mixture. To finish a whole cycle, it starts a new detritylation step. After it, all the steps will occur again until all the bases be coupled to finish the synthesis of the programed oligonucleotide. After the conclusion of the DNA strand the solid material from the column is submitted to a work up where the oligonucleotide will be cleaved from the solid support such as the $\beta$-cyanoethyl and the protected amino groups on the bases (benzoyl groups for $A$ and $C$, N6 and N4 respectively and isobutynyl group for G in N2). The cleavages are done submitting the sample to an aqueous ammonia solution at $50-60{ }^{\circ} \mathrm{C}$ for $5-6 \mathrm{~h}$. The ammonia attacks the 
carbonyl group of the ester and through an addition-elimination reaction is formed a new amide bond and the $3{ }^{`}-\mathrm{OH}$ on the DNA strand side. The $\beta$-cyanoethyl groups are removed due to the ammonia deprotonation at the acidic proton adjacent to the cyanide bond.

It is also possible to synthesize the DNA strand as "DMT-ON", which means the final nucleotide remains protected. This is a good advantage for purification, once the bulky group will provide a slow mobility through the chromatographic methods (RP-HPLC or gel electrophoresis) separating better the product from failure and shorter strands. The DMT group can be further easily removed by acidic treatment performed during desalting.

\subsubsection{UV/Vis spectroscopy}

UV/Vis spectroscopy is a technique which measures the absorption of visible (or ultraviolet) light and its behaviour based on excitation of electronic levels at certain wavelength, $\lambda$. Absorption spectroscopy provides resolutions for complex structural purposes and for simple applications, as for example, the determination of concentration for oligonucleotides. ${ }^{[4]}$

The incident light (I) penetrates through the sample and the fraction of absorbed light $\left(-\frac{\mathrm{dI}}{\mathrm{I}}\right)$, has a $I_{0}$ intensity, given by a certain $\lambda$ and dependence of concentration, $c$, and path length, I.

$$
-\frac{\mathrm{dI}}{\mathrm{I}}=\mathrm{c} \varepsilon^{\prime} \mathrm{dl}
$$

The molar extinction coefficient is given by $\varepsilon^{\prime}$. It is a constant value and independent of the concentration, specific for each molecule. Such value is integrated and converted to log base 10 , giving the equation:

$$
A(\lambda) \sim \log \left(\frac{I_{0}}{I}\right)=c \varepsilon(\lambda) l
$$

Where $\varepsilon=\varepsilon^{\prime} \frac{1}{2.303}$ and $A$ is the absorbance, the equation above is known as the Beer-Lambert Law. This law also brings dependency of concentration, where accurate measurements must obey a range from 0.1- 2.0 absorbance units (a.u). ${ }^{[4] \text { page365 }}$

UV/Vis measurements performed at $260 \mathrm{~nm}$ can also be used to estimate the concentration for DNA samples based on values of extinction coefficient for nucleobases. Further details about the calculation and methods that can be adopted are discussed with the results. 


\subsubsection{Thermal analysis}

Thermal denaturing analysis is a technique that monitors absorbance as a function of temperature. This technique can be applied to determinate the melting point of peptides, proteins and oligonucleotides. The information presented here is focused on the latter. Changes in the DNA conformation can be monitored by thermal analysis (melting and reannealing) and detected by hyperchromism effects, which brings an increase of $A$ when DNA denatures. This occurs because of stacking interactions of the bases during the double helix conformation causing a lower absorbance intensity than the respective separated single strands. The hyperchromism effect normally is around $15-20 \%$ during the melting process and can be used to analyze the relative stability of the duplex (ds-DNA) versus single strands (ssDNA) individually separated. ${ }^{[4]}$ It also can provide information concerning molecules interactions with DNA (interactions by $\pi$ stacking or hydrogen interaction of molecules in major and minor grooves with $d s$-DNA), metal interactions and distortions on DNA helix and how such additives can provide extra stabilization (or instability) for systems (duplexes, triplexes, quadruplexes) and their respective conformations.

The absorbance is recorded at specific wavelength ( $260 \mathrm{~nm}$ for duplexes, $295 \mathrm{~nm}$ for triplexes and 240 and $295 \mathrm{~nm}$ for quadruplexes are recommended, together with another wavelength where no interaction should take place, as a blank and control value) as a function of temperature, giving a higher (melted strands, ss-DNA) and lower (annealed system) levels in $Y$ axis (absorbance) in a sigmoidal shape curve profile. The width of the melting transition is important, as sharper the transition is, it can be observed an absorbance quite constant on temperatures near (above and below) the melting point region. The melting point is the region where the sample ratio is $50 \%$ melted and $50 \%$ annealed. Sharp melting transitions indicates a possible stable structure for both states processes, when heating (melting) and cooling (reannealing) the sample have a superimposable profile. This means the transition is kinetically reversible and for such cases the melting point determination is not complicated, as a sharp transition is simpler to analyze. ${ }^{[60]}$

Bimolecular and tetramolecular systems can be used to present slow kinetics for associations and dissociation processes. Due to this, it is possible to observe non superimposable curves for heating and cooling steps, such phenomenon is called hysteresis and indicates different kinetic paths for melting and annealing. The hysteresis depends on $\mathrm{T}$ gradient and indicates evidence of aggregated systems which makes more difficult to determinate the melting point. Neither heating or cooling process corresponds to $\mathrm{Tm}$, been the $\mathrm{Tm}$ value obtained always 
underestimated or overestimated. The most common factors that affect the melting curve are, besides temperature, a) the composition of the oligonucleotide and b) salt concentration. ${ }^{[61]}$

a) The oligonucleotide composition: the nucleotides sequences presented in a strand establish influences on the melting point (rich A-T regions tend to melt earlier than G-C regions) and also on structure, as the case for hairpin (an intramolecular system where the single strand is self-complementary) or triplexes (produced by systems $2 A+B$, for example). ${ }^{[62]}$

b) Salt concentration: The ionic strength has a direct dependence on the width and melting point. ${ }^{[63]}$ There are also differences on concentration, and between cations monovalent $\left(\mathrm{Na}^{+}, \mathrm{K}^{+}, \mathrm{Li}^{+}\right)$and bivalent metals such as $\mathrm{Mg}(\mathrm{II})$. How the cations can stabilize differently a DNA duplex and even promote high ordered structures formation (triplexes for example) when sequences and molecularity are also under agreement.

\subsubsection{Determination of the melting point}

There are two well known methods to determinate the melting point, Tm, through thermal analysis by UV/Vis spectroscopy, which are a) First derivative method and b) Folded fraction method. Both contain advantages and disadvantages summarized below and the choice of the method has to be based on the type of the system and on the complexity of the melting curve. ${ }^{[4,60]}$

a) First derivative method:

This method is a popular in the literature and it is applicable to all types of systems and structures, independent of molecularity. For comparisons between systems, it is commonly presented the data normalized, where the maximum $\left(A_{1}\right.$, melted) and minimum $\left(A_{0}\right.$, annealed $)$ absorbance values obtained in a melting curve are assigned as 1 and 0 respectively $\left(\left(A-A_{0}\right) /\left(A_{1}-\right.\right.$ $\left.A_{0}\right)$ ). The middle point between $A_{1}$ and $A_{0}$ ( 0.5 normalized absorbance) is theoretically given as the point were $50 \%$ of the sample is melted and the another half is annealed and is designated as the melting point. The determination of the melting point value thus calculates the first derivative of the melting curve, which is a function of absorbance ( $A$, axis $y)$ by temperature (axis $\mathrm{x}),\left(\frac{\mathrm{dA}}{\mathrm{dT}^{-1}}\right)$. The maximum of the first derivative is equal to Tm only for systems with intramolecular equilibrium, been for bimolecular processes $T_{\max }=\sqrt{2-1}(0.414$ instead of 0.5). A sharp transition allows a more precise melting point value and $\mathrm{T}_{\max } \approx \mathrm{T}_{\mathrm{m}}$. Such an "approximation" has $\pm 0.1^{\circ} \mathrm{C}$ precision. Depending on how wide is the transition, the 
difference between these values is more significant and a loss of accuracy can be related to the need of smoothing the curve (in cases of complex profile) to obtain the maximum on the first derivative. Systems containing two melting points (the case of intermediate secondary structure or triplexes) presents two maximum values for first derivative as well (Figure 23).

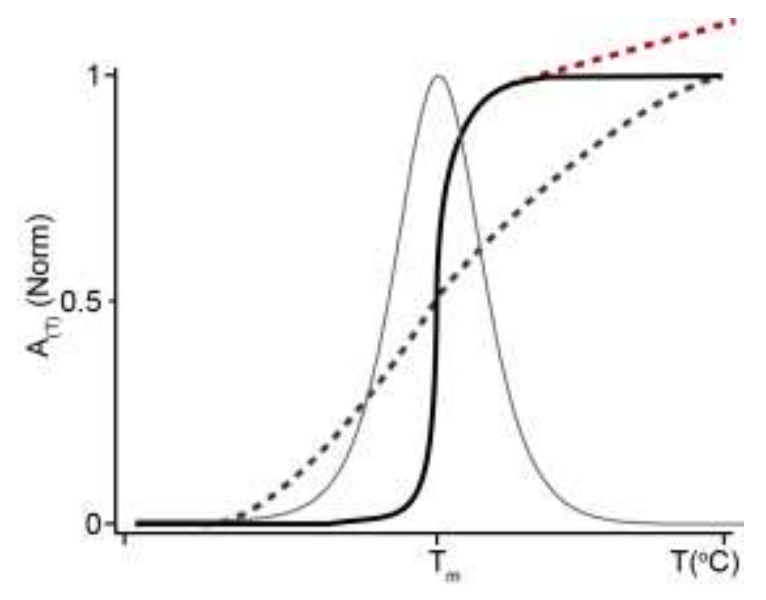

Figure 21. Melting curve profile for sharp $d s$-DNA transition (solid black line) and respective first derivative (solid gray line) it is possible to see, $T_{\max } \approx T m$ for sharp transitions, $d s$-DNA deviations due to evaporation (red dashed line) and ss-DNA (dashed gray line). ${ }^{[4,60]}$

b) Folded fraction method:

This method determines an upper and lower baseline, which will correspond to $100 \%$ folded $(\theta=1$, annealed $)$ and $100 \%$ unfolded $(\theta=0$, melted) fractions of the sample, respectivelly. The middle line between these baselines corresponds to the intercept in $Y$ axis (not absorbance as measured, but folded fraction, $\theta$ ), being equivalent to a middle point where $50 \%$ is $\theta=1$ and $\theta=0$; in other words, the melting point.

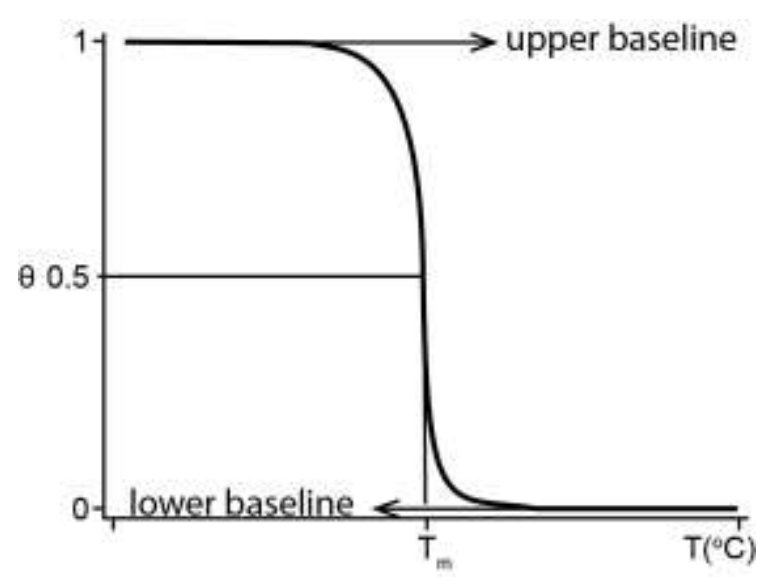

Figure 22. Melting curve for a sharp transition and its melting point determined by folded fraction method, indicating lower and higher baseline selected. ${ }^{[61]}$ 
The method has the advantage of accuracy in comparison to the first derivative method, where the maximum of the curve, Tmax, is just approximated to Tm. For the folded fraction method, at $\theta=0.5$, the temperature value is equivalent to the melting point, independent of system molecularity. Although, it is worthy to pay attention that the choice of the baselines (the points determined as maximum and minimum levels) are subjective and such choice provides slope dependency. Such values will be submitted to conversion from $A_{(\mathrm{T})}$ to $\theta_{(\mathrm{T})}$ as shown:

$$
\theta_{(\mathrm{T})}=\frac{\mathrm{L}_{0(\mathrm{~T})}-\mathrm{A}_{(\mathrm{T})}}{\mathrm{L}_{0(\mathrm{~T})}-\mathrm{L}_{1(\mathrm{~T})}}
$$

Where $L_{0(T)}$ and $L_{1(\mathrm{~T})}$ are values for lower and upper baseline, respectively, and are provided according to the slope of linear regression from the chosen curve range from upper and bottom levels on the melting curve.

This means that all absorbance values measured in the curve, when converted to folded fraction, are submitted to conversion based on such slope dependence. Based on the system and its complexity, the values subtracted can result in a loss of data which can changes the curve profile (Figure 23d). For simple systems, unimolecular or bimolecular, without intermediates and with superimposable paths for melting and annealing processes (kinetically equal), it can be observed that the data conversion does not cause drastic changes on the melting profile such as on the melting point. This was concluded based in comparison of values for Tm obtained between both methods and other methods given by the software, (leastsquare, $2^{\text {nd }}$ derivative and one-point) which show a lower discrepancy between them. 

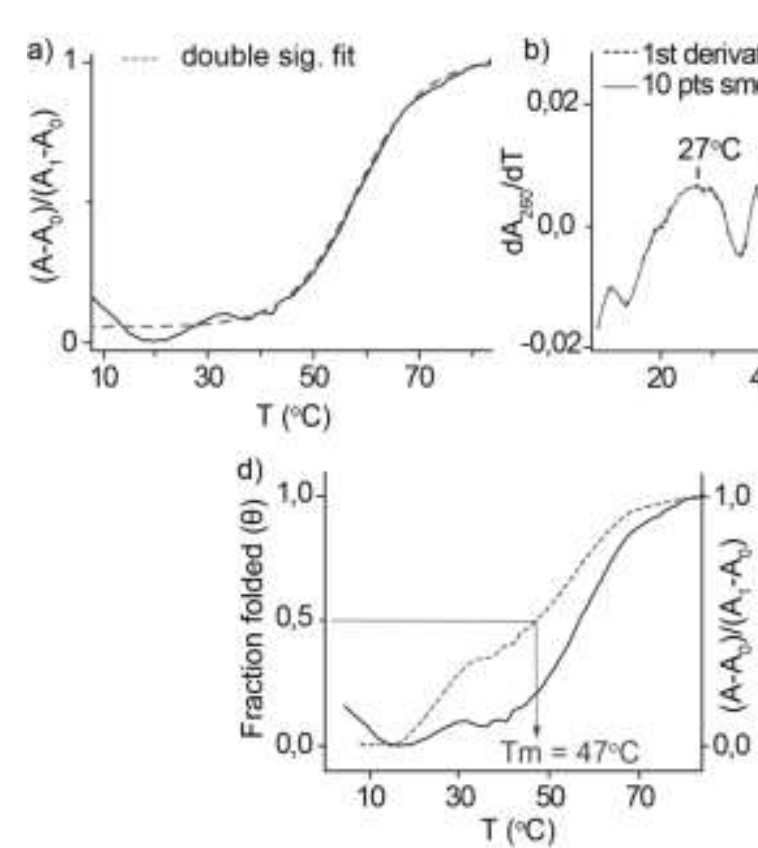
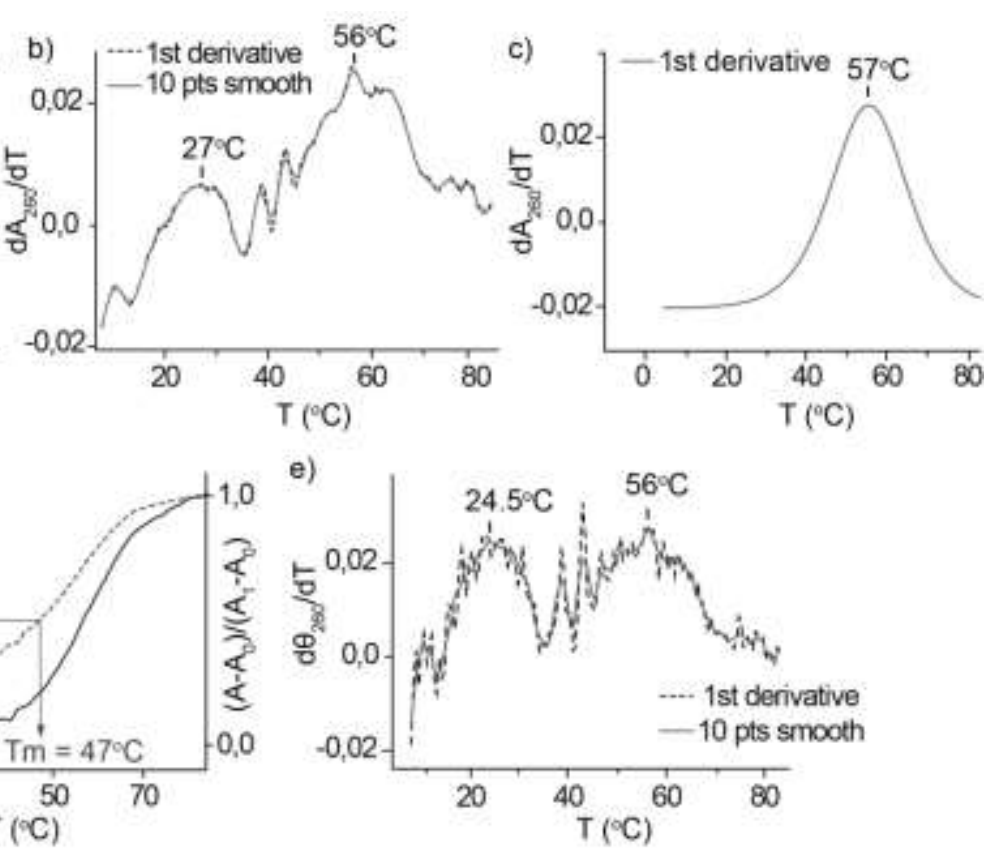

Figure 23. Examples of treatments for thermal analysis curve for a sample (see section 2.2.4 for sample details and results) of our own work. ${ }^{[37]}$ a) Original melting curve profile (solid black line) and after double sigmoidal fit (dashed line); b) Melting points of the curve presented in a) based on the first derivative method (dashed line) and smoothed (solid line); c) First derivative method for curve treated by double sigmoidal fit (excluding intermediates state effects from the curve). d) Melting curve (solid black line) and its respective profile treated by folded fraction method (dashed line) indicating the melting point value obtained by folded fraction conversion ( $\mathrm{Tm}=\mathrm{T}$ at $\theta=0.5, \mathrm{Tm}=47^{\circ} \mathrm{C}$ ) and e) First derivative of the curve after conversion by folded fraction method.

The melting curve and its respective profile after treatment of the values by folded fraction method can be seen on Figure $23 \mathrm{~d}$ and indicates that, despite the melting curve profile changes, if applied the first derivative the value obtained does not alter so much from those obtained by first derivative method. Significant difference was observed for the melting point of the intermediate state (first melting point, $-2.5^{\circ} \mathrm{C}$, after folded fraction values conversion).

For complexes systems containing intermediates, as triplexes or systems with pronounced hysteresis and especially systems which deviates from "all-or-none" model, it is possible to observe more than one melting point. The choice of upper and lower baseline have to be done separately for each "part" of the curve, correspondent to each transition. If this is not done, the curve profile changes after conversion (Figure 23d) showing a melting point determination for the main or both transitions, with significant deviations. The independent treatment for 
each transition also complicates the comparison with other curves, if is desired to show them together.

\subsubsection{Considerations about nucleation, zippering and the "all-or-none" model for the melting process}

When a sharp transition for melting curve indicates an abrupt change, this process is described as "cooperative" and does not contain intermediate states, assuming as possible only fully annealed double helix or completely separated single strands. This model is a good approximation for small oligonucleotides and it is known as an "all-or-none" melting model, or "zipper" model. The model assumes that the formation of a double helix can starts at any point between paired complementary single strands and from the first base pairing the hydrogen bonding can develop the whole network. ${ }^{[4]}$

The base composition affects the DNA melting, since it is dependent of the nucleotide sequence. The dependence of enthalpy and entropy for G-C and A-T base pairs (ignoring vertical stacking differences) are:

$$
\begin{aligned}
\Delta \mathrm{H}^{\circ}{ }_{\mathrm{GC}} & =\Delta \mathrm{H}^{\circ}{ }_{\mathrm{AT}}(1+\mathrm{a}) \\
\Delta \mathrm{S}_{\mathrm{GC}}^{\circ} & =\Delta \mathrm{S}^{\circ}{ }_{\mathrm{AT}}(1+\mathrm{b})
\end{aligned}
$$

Where $\mathbf{a}$ and $\mathbf{b}$ are parameters. At the melting point for sharp transitions, $\Delta G^{\circ}=0$, which makes $\mathrm{T}_{\mathrm{m}}=\frac{\Delta \mathrm{H}^{\circ}}{\Delta \mathrm{S}^{\circ}}$, combining both equations it is given then:

$$
T_{m}=\frac{\Delta H_{A T}^{\circ}\left(1+a \chi_{G C}\right)}{\Delta S_{A T}^{\circ}\left(1+b \chi_{G C}\right)}
$$

The equation above indicates a linear dependence of $\mathrm{Tm}$ on the G-C base pair amount and can be confirmed experimentally by varying the $\chi_{G C}$ presented in a sequence. ${ }^{[64]}$ Rich regions in G-C base pairs in a duplex are more stable and melt before A-T rich regions. ${ }^{[4]}$ Based on this, it is possible to conclude that the model "all-or-none" for melting process is not applicable to DNA. E. Reich et al. performed studies where it was synthesized oligonucleotides with local regions (the end of the strands in a duplex) more stable or less stable than natural double helix analogue. Guanine nucleotides (dGTP) were replaced by dI (dITP) to create weaker base pair I$\mathrm{C}$ providing weaker ends on the double helix, producing lower $\mathrm{Tm}$ than the value obtained for natural duplex. It was possible to observe that the melting is a two step transition, where the weaker ends melt first than the middle part (stabilized). For strong ends, the nucleobase $C$ was replaced by $5 \mathrm{BrC}$, base $\mathrm{T}$ replaced by $5 \mathrm{BrU}$ and 2,6-diaminopurine for $\mathrm{A}$ bases. It produced a melting transition also with two steps as the another case, but here a higher Tm value was obtained with the middle part melting first (weaker) than the stronger ends. ${ }^{[65]}$ 
Melting transitions where intermediates can not be observed by changes in absorbance (it varies according to the temperature rate, base sequence, ionic strength and concentration) can be studied by hydrodynamics methods as for example viscosity measured as function of temperature. It is possible to detect the "hidden" Tm for intermediates and its temperature dependence observing small portions of melting by increasing of $T$, causing disruptions which are too small to be detected by changes in absorbance. These melted regions can form hairpin and also gliding of the DNA strands in the melted areas. ${ }^{[4,33]}$

The "all-or-none" melting model assumes a single nucleation step and it can be in any point between two aligned self-complementary strands. Kinetics of B-DNA melting are based on the base stacking (which is an intramolecular process) and in the Watson-Crick base pairing. Studies for systems with a sequence of nucleotides higher than 4 showed to be too complicated to be determined and the "zipper" model, despite simpler than reality, is used and satisfies the most of the kinetics situations. Cantor and Schimmel say "it seems more plausible, from actual rates and activation energies involved, that the first base pair exist in a rapid pre-equilibrium, that the rate-limiting step is the formation of the second base pair". [33]page 1219. The zipper model establish a linear increasing for further rates based on the length of the duplex shows that such prediction does not happen experimentally and nucleation is spread over several base pairs, indicating that activation energies and rates are not dependent on length for double helix formation (thermodynamically the opposite, denaturation, decreases with increasing of length). ${ }^{[66]}$

\subsubsection{Fluorescence spectroscopy}

Photon absorption can promote an electron from ground state, $\mathrm{S}_{0}$, to an excited state, $\mathrm{S}_{1}$. The excited state has higher energy and excitations occur in a correspondent frequency of light. Once the electron relaxes and goes back from excited singlet state to $S_{0}$, such energy can then be released as light emitted in a process denominated fluorescence. There are other ways to lose the energy besides light emission, it can be for example through heat, or due to internal conversions caused by vibrational modes producing a triplet excited state, $T_{1}$. Triplet state relaxes to $S_{0}$ emitting through the process of phosphorescence, which has a longer radiative lifetime than fluorescence.

Fluorescence normally has a lifetime of $\sim 10^{-9}-10^{-8} \mathrm{~s}$, while the time scales of absorbance and CD spectroscopy are $\sim 10^{-15} \mathrm{~s}$. It is a more sensitive technique and can be used to follow 
conformational changes and binding of ligands, since at $10^{-8} \mathrm{~s}$, it can occur protonation, deprotonation and solvent-cage changes. Each different process to release energy and promote the electron return to $S_{0}$ is valuable and provides different information concerning the molecule properties. Fluorescence embraces processes related to translational or rotational motion. Internal conversions due to vibrational relaxations occur in a rate $\left(\mathrm{K}_{\mathrm{ic}}\right)$ which in general increases according to temperature increasing. Fluorescence intensity is dependent on temperature, which it is important to highlight that it is not found in any other spectroscopic effect. The solvent effect is also different for absorption and emission. Such properties for absorbance and emission can be monitored by UV/Vis. ${ }^{[4,67]}$

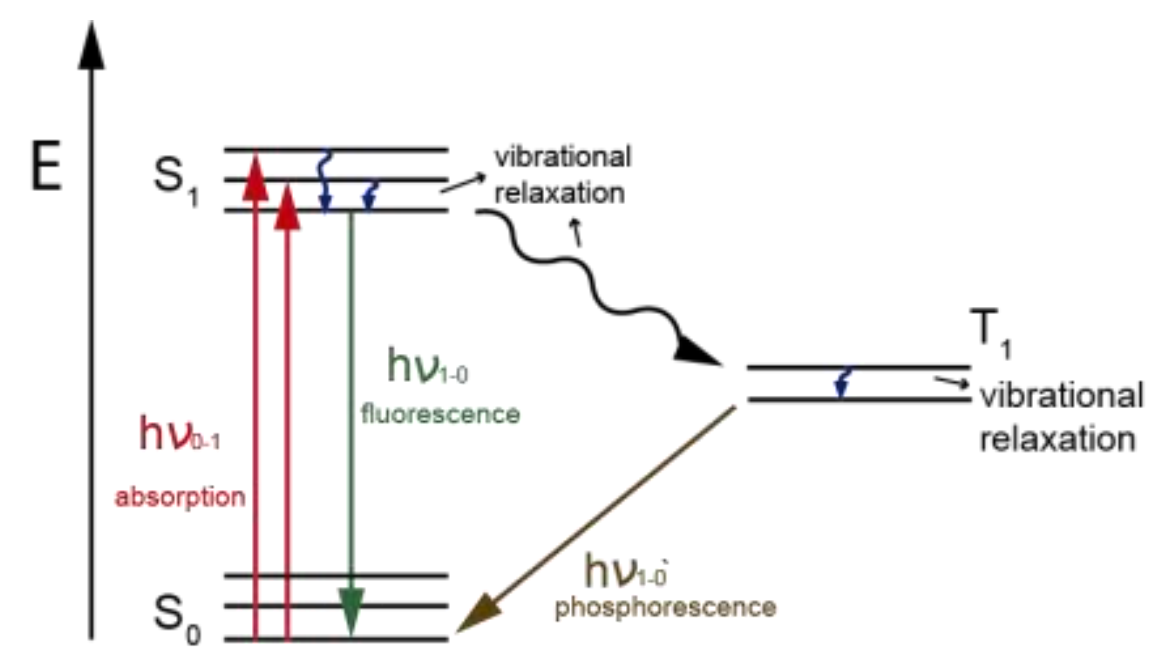

Figure 24. Scheme for photon excitation and emission and vibrational relaxations. ${ }^{[67]}$

The fluorescence can be based on the quantum yield, $\Phi_{F}$, which is a ratio between the light emitted and absorbed in a certain wavelength. The quantum yield can be given by the following equation:

$\Phi_{\mathrm{F}}=\Phi_{\mathrm{R}} * \frac{\mathrm{I}_{\mathrm{F}}}{\mathrm{I}_{\mathrm{R}}} * \frac{\mathrm{OD}_{\mathrm{R}}}{\mathrm{OD}_{\mathrm{F}}} * \frac{\mathrm{n}_{\mathrm{F}}^{2}}{\mathrm{n}_{\mathrm{R}}^{2}}$

$\Phi_{\mathrm{F}}=$ Sample quantum yield (fraction of the excited singlets that decay by fluorescence)

$\Phi_{\mathrm{R}}=$ Standard quantum yield

$\mathrm{I}_{\mathrm{F}}=$ Sample integrated of fluorescence emission

$I_{R}=$ Standard integrated of fluorescence emission

$\mathrm{OD}_{\mathrm{F}}=$ Sample optical density (Normalized absorbance according to the optical path)

$\mathrm{OD}_{\mathrm{R}}=$ Standard optical density

$\mathrm{n}_{\mathrm{F}}=$ Sample refractive index of solvent

$\mathrm{n}_{\mathrm{R}}=$ Standard refractive index of solvent

Another alternative for quantum yield measurements is through a ratio of the decay rates: 


$$
\Phi_{F}=\frac{\tau_{F}}{\tau_{R}}
$$

$\tau_{\mathrm{F}}=$ fluorescence decay time

$\tau_{R}=$ radiative lifetime of excited singlet level

There are different ways to generate fluorescence in potential samples, but they all need at least one labelled molecule, called fluorophore. Fluorophore are compounds with ability to absorb and emitting energy as light (chromophores absorb light but do not release energy as light emission, it releases energy through different relaxation processes) in a specific wavelength. Decreases in fluorescence intensity are denominated "quenching". Among several techniques based on quenching, it highlight the energy transfer from a fluorophore to another or to a non-fluorescent molecule. The quenching through the formation of a non fluorescent molecule is known as "contact-quenching" or "ground-state complex". The reaction should be followed by UV/Vis spectroscopy, where change in the spectrum can be observed while in FRET mechanism no change in the absorbance spectra are noticed. FRET is a type of energytransfer resonance, which will be discussed on the next subtopic. ${ }^{[67]}$
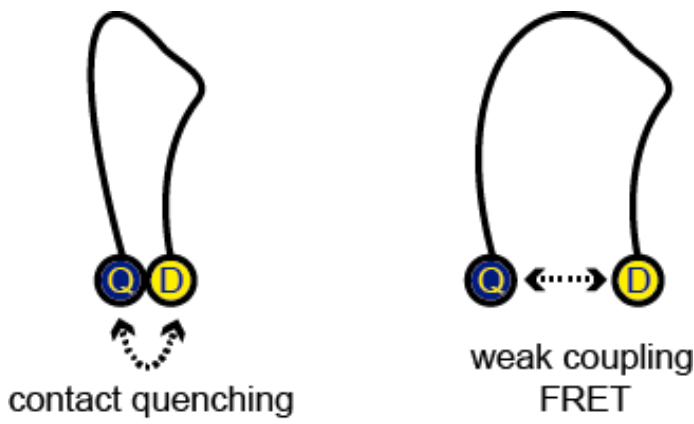

Figure 25. Figurative scheme for contact quenching and weaking coupling mechanisms. ${ }^{[67]}$

The percentage of quenching can be calculated based on the relationship of quenched and non-quenched values. For cases where is obtained a ground-state complex for example, the percentage quenching is given by the signal of the non-quenched sample divided by the signal of quenched sample (both values with buffer blank subtracted), multiplying the result by 100 and further, subtraction of the obtained value from 100. ${ }^{[67] \text { page36 }}$

\subsubsection{Fluorescence resonance energy transfer, FRET}

Fluorescence resonance energy transfer is also called Förster resonance energy transfer because of the developer of the technique, Theodor Förster. The latter nomenclature is preferable because this techcnique is actually based on a nonradioactive energy transfer, even 
if both chromophores used are fluorescent. The mechanism of energy transfer concerns to two chromophores, one as donor and another as acceptor. The donor fluorophore goes back to ground state when transfer energy to the acceptor chromophore (can be a fluorophore or not), which will raises to higher vibrational levels for excited singlet state and release the transferred energy as emitted light, or heat in case of non-fluorescent acceptors. ${ }^{[67]}$

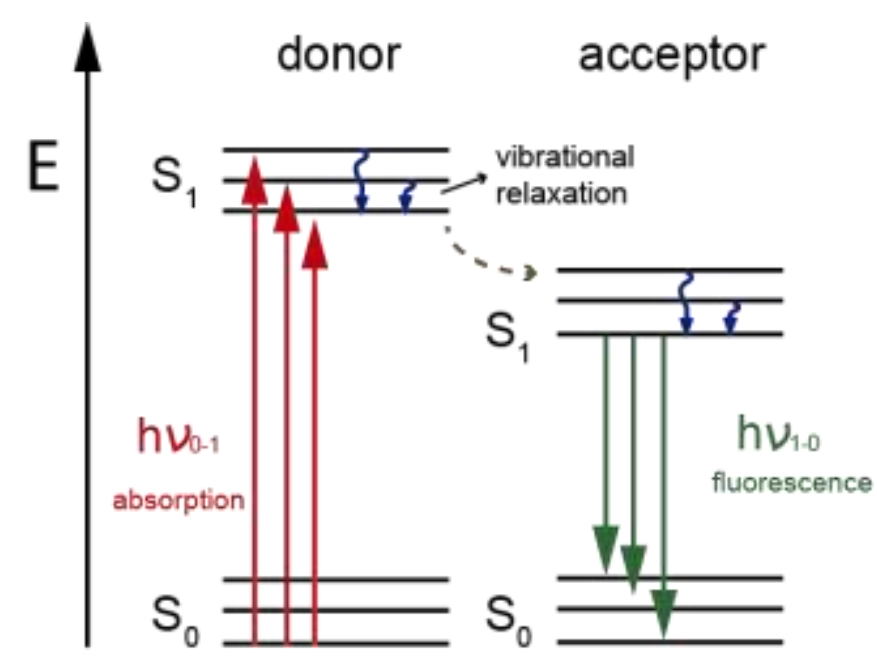

Figure 26. Scheme for photon absorption and emission of donor and acceptor. ${ }^{[67]}$

The energy transfer is due to non-radiative process, through a dipole-dipole moment which depends on the molecules orientation and it is limited by the distance between donor and acceptor, typically effective in a range of 10-100 ̊. If the distance is large, the energy transfer is low and the emission measured from the acceptor is reduced. The separation distance is important for FRET measurement, as the energy transfer is dependent to a distance $R^{-6} .{ }^{[68]}$ The FRET efficiency can be calculated following the equation below:

$\mathrm{E}=\frac{\mathrm{K}_{\mathrm{T}}}{\left(K_{T}+\frac{1}{\tau_{D}}\right)}$

where $K_{T}=\left(\frac{1}{\tau_{D}}\right)\left(\frac{R_{0}}{R}\right)^{-6}$; which has been replaced in the equation above and rewriting, we can have the efficiency of energy transfer yield as:

$\mathrm{E}=\frac{\mathrm{R}_{0}^{6}}{\left(R_{0}^{6}+R^{6}\right)}$

$\mathrm{R}_{0}=$ transfer distance

$\tau_{D}=$ donor lifetime without presence of acceptor

$\mathrm{K}_{\mathrm{T}}=$ rate of transfer

$\mathrm{R}=$ distance between the two chromophores 
It is also important to note other conditions that can affect FRET such as concentration, changes on $\mathrm{pH}$, temperature and proper choice of the chromophore pairs. Emission donor spectrum has to overlap the absorption acceptor spectrum, to produce an effective energy transfer. The emission process always happens in a lower energy than the absorption process, this is because there is always a loss of energy during the process and due to it, the emission always occur in a longer wavelength than absorption.

\subsubsection{Types of FRET}

DNA samples having FRET between chromophores can have different approaches as described below: ${ }^{[67]}$

\section{a) Strand Displacement}

It is based on a DNA duplex where each single strand contains one chromophore each, where the donor and acceptor chromophore are in the end positions ( $3^{`}$-end and $5{ }^{`}$-end). Contact quenching due to the proximity between donor and acceptor results in no fluorescence being emitted. The addition of a third strand, as a target strand complementary to one of the single strands, will cause displacement and formation of another double helix, more stable and favorable. Once this happens, the donor and acceptor are spatially separated from each other and fluorescence increases by donor emission.

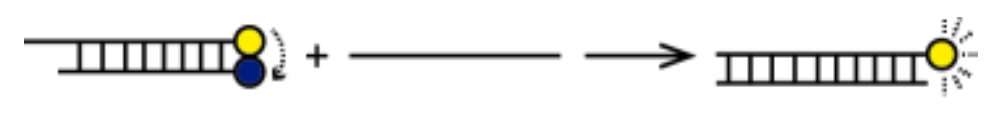

Figure 27. Scheme for strand displacement by addition of a target strand. ${ }^{[67]}$

b) Adjacent probes

The use of adjacent probes involves two single oligonucleotide strands, where one is labelled with a fluorophore at $3^{`}$-end and the other one containing an acceptor chromophore at $5^{`}$-end. A third strand equally complementary to both shorter labelled strands is inserted on the system. This third strand is designed to hybridize with both single strands in a way to place the chromophores whith a short distance between them, enough to produce energy transfer. Before the hybridization, the single strands containing the chromophores are free in solution (respecting concentration conditions diluted enough to kept them apart) and does not present 
energy transfer. The FRET efficiency depends on the designed distance such as the chromophores properties.

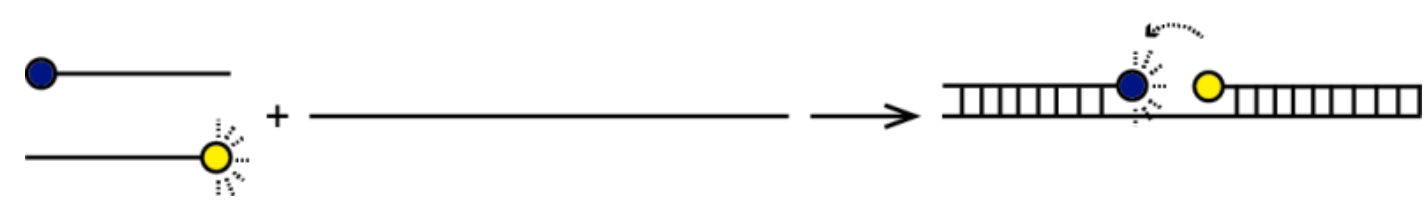

Figure 28. Scheme for adjacent probes where single strands containing donor and acceptor are hybridized with a longer complementary strand in a way that both chromophores are placed closed to each other allowing weak coupling of energy transfer. ${ }^{[67]}$

c) Molecular Beacon probes

The stem-and-loop structures are based on nucleotide sequences that are partially complementary (on their sides) producing an "arm" sequence (hairpin) with a loop part, not hybridized in the middle. Each end of the oligonucleotide contains a chromophore, a fluorophore donor and a non-fluorophore acceptor. Since the sides are hybridized, the chromophore pair has through contact quenching decrease of fluorescence. Addition of a DNA or RNA target strand promotes a reorganization and the formation of a longer and stabilized duplex with the target probe. The duplex formed keeps a distance between donor and acceptor which produces fluorescence by energy transfer.

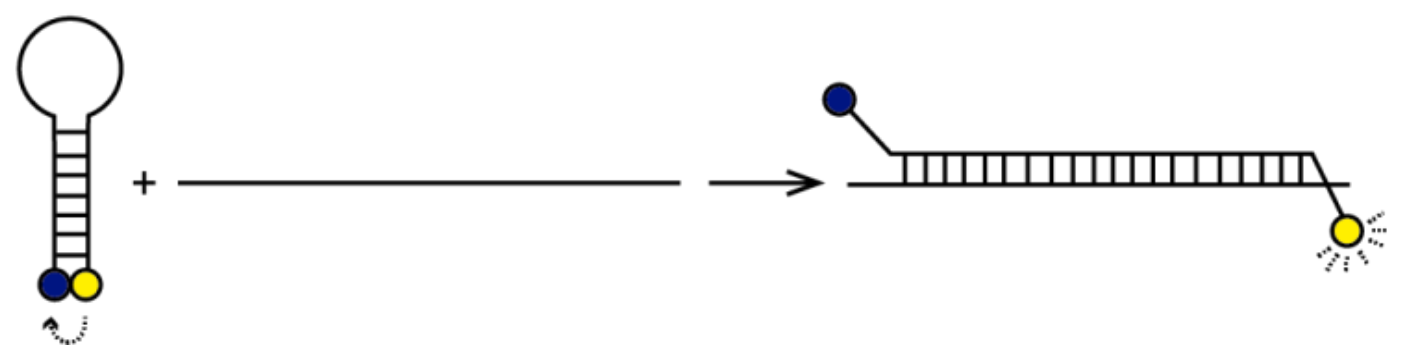

Figure 29. Scheme for a molecular beacons probe, a hairpin structure containing a groundstate complex between donor and acceptor and a further unfolding of such structure due to addition of complementary strand promoting emission of the donor fluorofore caused by the distance of the acceptor. ${ }^{[67]}$

d) TaqMan $^{\circledR}$ probe

In this case, a single strand containing the donor and acceptor, one at each end of the strand (5`-end containing donor and non-fluorescent acceptor) and having appropriated length to obtain energy transfer translated as a low fluorescence emission due to quenching. Once the 
complementary strand and Taq DNA polymerase are added, the DNA hybridization and polymerase chain reaction primer occur at the same moment. The strategy applies to the fact that the 5-nuclease activity cleaves the sample and separates the donor from the acceptor. The donor fluorophore once is free of the FRET effect releases energy as can be observed by the increase of the fluorescence. Intensity of the fluorescence corresponds to the amount of donor fluorophore that has been cleaved, until all is consumed and a maximum of emission is reached.

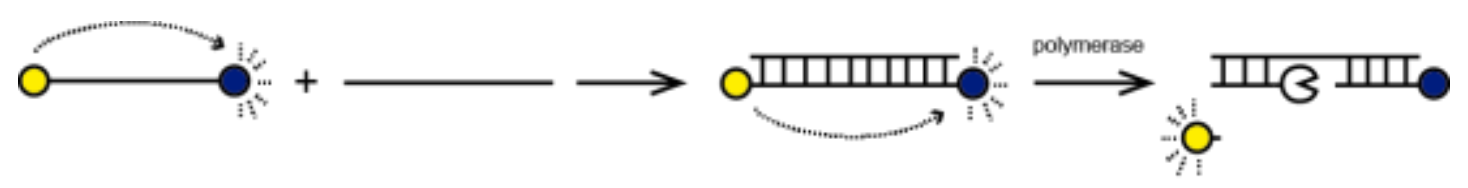

Figure 30. Scheme for TaqMan ${ }^{\circledR}$ probe promoting cleavage of system with donor-acceptor pair. ${ }^{[67]}$

\subsubsection{Circular Dichroism Spectroscopy}

Circular Dichroism $(C D)$ is sensitive to the difference between left $(\Delta \mathrm{L})$ and right $(\Delta R)$ polarized light, providing change of absorption, $\Delta \varepsilon$. It only arises at wavelengths of light absorbed by chiral molecules, which means the $\Delta \varepsilon$ depends on asymmetry (for molecules without plane of symmetry, no mirror image). It is a useful spectroscopy for conformational studies of proteins and oligonucleotides, providing signatures for secondary structures in solution. ${ }^{[4]}$

For DNA the chirality comes from the sugar backbone and how the helical structure is established. The CD spectrum of electronic transitions is directly linked to the absorption produced by the bases, A, T, C and G. The way in which the bases stack provides strong contribution to the technique sensitivity and it is in a range between $190-320 \mathrm{~nm}$. It is possible to distinguish the DNA conformation, for example the identification of duplexes as $A, B$ or $Z$ forms, (such as the transitions between B form to A form, which causes dehydration or promotes to $\mathrm{Z}$ form, that is related to salt concentration), triplexes and quadruplexes (and its respective folding type). ${ }^{[69]}$

Focusing on duplexes, $A$ and $B$ forms are right handed while $Z$ form is left handed. The transition between A and B forms is possible due to the deoxyribose's backbone, which can adopt C3 'endo ( $A$ form) or C2 ${ }^{`}$ endo ( $B$ form) pucker. The B form is characterized by a negative peak at approximately $245 \mathrm{~nm}$ and a positive peak around $280 \mathrm{~nm}$, while the A form has as 
characteristic signature of a sharp negative peak at $210 \mathrm{~nm}$ and a positive peak around $260 \mathrm{~nm} \cdot{ }^{[70,71]}$

The double helix environment is chiral and it can imprint onto the ligand incorporated (in the case of artificial nucleotides). Normally the chirality transfer for the ligand can be observed at wavelengths closer to $320 \mathrm{~nm}$, the exact value depends on the ligands properties.

\subsubsection{Electrospray ionization mass spectrometry, ESI-MS}

Mass spectrometers provide a mass-to-charge ratio of ions in vacuum. Electrospray ionization mass spectrometry is also known as "soft ionization", produces less fragmentation. The sample can be an analyte in aqueous solution, normally combined to methanol or acetonitrile and introduced through a syringe with stainless steel capillary in the presence of an electric field. The electrospray is generated from high voltage that creates a mist of droplets. The droplets are charged and contains excess of ions of one polarity due to the strong electric field that causes movement of the ions in the liquid. The droplet polarity depends on the sign of the applied potential. For organic and inorganic molecules is conventionally positive charges, producing $[\mathrm{M}+\mathrm{nH}]^{\mathrm{n}+}$ and $[\mathrm{M}+\mathrm{Na}]^{+}$charges. For oligonucleotides a negative ion mode is used, since the DNA is already naturally negatively charged, due to the phosphordiester-backbone. Interpretation of peaks and details comes further on results. ${ }^{[72]}$

After droplet formation it is important elucidate details of droplet production. The droplets will travel through the capillary and during the process the solvent evaporates. It causes a decrease on the droplet radius until reach the Rayleigh limit, the critical radius size. At this point, the Coulomb repulsion between the charges is stronger than the cohesive forces and it causes the droplet "explosion" in an asymmetrical way producing small derivatives droplets enriched with the ions from the original droplet. The process undergoes to evaporation again until a level where the single ions are isolated in an environment with counter ions and solvent molecules.

a) Electrospray for DNA

DNA duplexes were first detected intact by ESI-MS in 1993 and DNA adducts for non-covalent interactions right in the next year. ${ }^{[73,74]}$ Other techniques applied would be Crio-ESI and MALDI. A comparison between MALDI and ESI can be resumed in table 1 . The best advantages of ESI-MS are the extended mass range, sensitivity and resolution. A major concern in ESI 
measurement for DNA is how to suppress the fragmentation. There are differences based on the type of equipment, reporting changes on voltage and conic voltage are ways to significantly reduce the fragmentation. Despite technical parameters dependent on the equipment, salts of sodium and potassium give a wide distribution of DNA adducts detected. For this reason, 20-30 $\mu \mathrm{M}$ DNA duplexes are better detected using 100-150 mM ammonium acetate $(\mathrm{pH} \sim 8.3)$.

The negative ion mode for DNA presents a set of multiple negatively charged species. Considering for example a 12-mer, the sequence contains in its backbone 11 possible charged $\mathrm{PO}^{-}$groups, which provide such set of negative charged species. The possible peaks are related to the possibility of deprotonations based on $\mathrm{m} / \mathrm{z}$ ratios. To calculate $\mathrm{m} / \mathrm{z}$ ratio is necessary to consider the molecular weight of the oligonucleotide and subtract the number of deprotonations, to then divide the value by such deprotonation number giving peaks $[M-n H]^{-n}$. It is possible to observe peaks as $[\mathrm{M}-\mathrm{nH}+\mathrm{Na}]^{-\mathrm{n}+1}$ and similar peaks to other cations(I) or (II). If it is provided through experimental measurement a $\mathrm{m} / \mathrm{z}$ value and it is desired to know to which charge it is referred, the interpretation can be done through isotopic peak distribution. The isotopic peak distribution, $\mathrm{m} / \mathrm{z}$, to assign the charges works according to the fact that the space between the isotopic peaks in the $\mathrm{m} / \mathrm{z}$ scale is given by inverse of the charge: $1 / \mathrm{z}$. Nevertheless, it is essential a comparison between the isotopic peaks for experimental and theoretical values of $\mathrm{m} / \mathrm{z}$.

Table 1. Comparison between MALDI and ESI-MS.

\begin{tabular}{|c|c|c|}
\hline & MALDI & ESI-MS \\
\hline Sensitivity & High (2 pmol) & High (250 fmol-10 pmol) \\
\hline Molecular weight & 300000 atomic mass units (amu) & Max. $100000 \mathrm{amu}$ \\
\hline Resolution & $\begin{array}{l}<600 \mathrm{Da} \text { (due to matrix signal), but } \\
\text { tolerant for salts and buffers }\end{array}$ & $\begin{array}{l}\text { Salts, buffers, additives. Limit } \\
\text { concentration } \sim 150 \mathrm{mM}\end{array}$ \\
\hline efficiency & $>50$ bases not efficient & $20-120$ bases \\
\hline Species detected & Parent ions & Multiple negative charged species \\
\hline
\end{tabular}

\subsubsection{Methods of purification for oligonucleotides}

There are several methods to purify oligonucleotides and proteins. Here will be presented only those related to the research and performed on the work. ${ }^{[59]}$ 
a) Gel Filtration:

The gel filtration method is based on size exclusion chromatography, containing a stationary phase normally composed of a gel matrix or a porous resin. Gel filtration can separate protecting groups, salts and short strands, which are retained on the gel matrix. The larger oligonucleotide is eluted through the gel filtration. An example is the Illustra NAP'M -columns by GE Healthcare Life Sciences. These are columns based on Sephadex ${ }^{\mathrm{TM}}$, a cross-linked dextran gel and it works by gravity for desalting, buffer exchange and purification of small DNA fragments. These filtration columns are limited to oligonucleotides with at least 10bp and concentrations lower than $1 \mathrm{mg} / \mathrm{ml}$. The recovery is around $90 \%$ and it presents a salt contamination minimum $3 \%$.

Another example are Glen-Pak ${ }^{\mathrm{TM}}$ cartridges, by Glen Research, which are based on a resin as stationary phase (composition not detailed by the fabricant). In this case, the DMT-ON oligonucleotides can be desalted and separated from failure and short strands since the $5^{\prime}$ DMT binds to the solid support on the cartridge. After proper washing of such impurities, the DMT group can be cleaved by a 2-3\% trifluoroacetic acid solution (TFA) and the oligonucleotide is eluted. This cartridges are limited to 150 bases for DNA and 60 bases for RNA.

\section{b) Fluorous affinity chromatography}

This chromatography is based on the high affinity of organic compounds highly fluorinated for other fluorinated compounds. The solid phase DNA synthesis can be performed carrying on the last base coupled a phosphoramidite with a fluorous tag (5'-DMT group containing a perfluorinated alkyl chain). That means, in case of failed strands, they will not be tagged. The crude material containing the oligonucleotide is then loaded on a fluorous column (containing resin that binds fluorinated organic groups) and the oligonucleotide is then bound to the solid support and all impurities such as salts and failure strands will elute. After proper washing, the oligonucleotide can be detritylated and lose the strong interaction based on the fluoro affinity, and then eluted from the column.

c) Polyacrylamide gel electrophoresis:

Gel electrophoresis is a method for DNA, RNA and proteins purification based on their size and chain length related to the movement of the charged molecules due to an electric field applied, causing a separation between the molecules on the crude sample. DNA has a sugar- 
phosphate backbone, which is negatively charged. The DNA is loaded in a gel, that means a matrix (agarose or polyacrylamide) with porous size controlled (on PAGE by the ratio of acrylamide/bis-acrylamide amount) placed inside of a chamber with buffer solutions and electrodes (a positive and a negative in each side) connected to a source of power. Once an electrical field is applied, it moves the negatively charged oligonucleotide through the gel (towards the electrode of opposite charge, in this case, the positive). Shorter strands move faster than longer strands, because of the porous size of the gel. This is called "sieving" phenomenon. The different sieving between the sequences provides distinct bands on the gel, each band containing the respective sequence according to its mobility inside the matrix. This method gives a high purity sample, although it compromises in yield and it is a quite timeconsuming technique despite being cheap. For DNA, there are some restrictions concerning secondary structures, as for example hairpin. The method requires buffer, which will favor the formation of such structures, without neglecting the dependence on the sequence to promote such possibility. There are other conditions to consider such as the temperature of the system, $\mathrm{pH}$, concentration and type of the buffers, gel composition (percentage of acrylamide/bisacrylamide to form smaller or bigger sized-porous). The matrices can be polyacrylamide (in case of 10-3000 bp) or agarose (50-20000 bp) and can focus on single strands (in presence of dissociating agents, as urea) or double strands (absence of dissociating agents). Native PAGE electrophoresis is focused on non-denaturing gels, for that it is also important to control the temperature during the gel running, which tends to heat because of the current running through the gel and buffer, been necessary a cooling system placed on the chamber. Denaturing gels are done in presence of urea and promote the unfolding of the structure, causing a linear-chain on the sequences such as also separating the complementary single strands. The respective bands to different separated components can be visualized by stains, UV/Vis shadowing or with the use of intercalators as dyes (as ethidium bromide for DNA, for example). It is also common the use of markers, which are composed by a mixture of known size molecules, been placed in parallel in extreme columns on the gel to help to characterize the bands by comparison of height and mobility.

d) High-performance liquid chromatography

High-performance liquid chromatography (HPLC) is a purification method that is also used to identify and quantify components in a mixture. Different from a chromatographic procedure by gravity on HPLC the system works under higher pressure and the columns contain smaller sorbent particles, which gives a better separation of the compounds. A standard HPLC equipment contains an injector for the sample, pumps to deliver solvents under pressure, a 
mixing system (to handle the sample into the mobile phase to be eluted on the HPLC column for separation process) and a detector, normally based on UV/Vis to provide a proportional peak respective to the amount of compounds been eluted from the column, which allows a quantitative analysis of the compounds. ${ }^{[75]}$

Reverse-phase HPLC is largely used for oligonucleotide purification and it is based on hydrophobicity. A crude material after solid phase synthesis might contain the desired strand synthesized but also failed strand, byproducts (modified nucleotide uncoupled) salts and short oligonucleotides sequences where each component has a different hydrophobicity. A HPLC column for DNA is normally based on hydrocarbon chains (Nucleodur ${ }^{\circledR} \mathrm{C} 18$ ) bound to a silica support with a defined pore size $(300 \AA$, particle size $5 \mu \mathrm{m})$. The longer the oligonucleotide sequence is, the more difficult is the purification such as the separation between strands with similar length. A good approach to purify sequences obtained by solid phase synthesis is to work on DMT-ON mode. The desired sequence will contains a 5 '-DMT group on the last base, which increases the degree of hydrophobicity of the strand and makes the elution slower, making a significant difference between the sequence and its fail strand (it will elute first due to a weaker interaction with the column) providing very distinct peaks and facilitating the purification. The gradient for purification, which means the ratio of solvents during elution plays an important role on the separation of components. For DNA a common solvent mixture used is MeCN+TEAA (triethylammonium acetate), where the system becomes more hydrophobic depending on the percentage of MeCN. Another difficulty for oligonucleotides purification is for sequences where it is possible to have secondary structures (hairpins, quartets, triplexes) which causes variations in mobility and hydrophobicity and produces broader peaks (overlapped components). To overcome this problem is advised to use an oven $\left(40^{\circ} \mathrm{C}\right.$, higher temperatures can damage the solid support of the chromatographic column and the gradient adopted will not respond properly anymore) to promote unwinding of the strand during elution. 


\section{Results}

\subsection{Aims of project (part 1) - Orthogonal self-assembly between trans-chelating, terpyridine, phenanthroline, other related ligands, and divalent metal species.}

Self-assembly through metal coordination is an efficient method to obtain high-ordered structures and, in supramolecular chemistry, this is a well developed approach with several successful examples ${ }^{[76]}$ producing catenanes, rotaxanes, and many other systems with different shapes and structures. ${ }^{[77]}$ Here we have as a main goal the introduction of a new type of ligand based on trans-chelating motif with the ability to form square-planar complexes

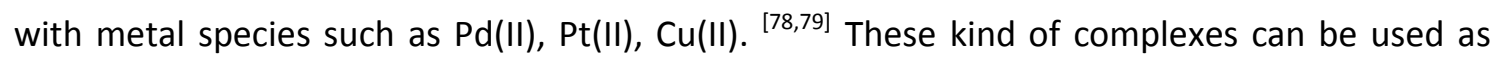
building blocks for further formation of high-ordered heteroleptic structures, upon addition of complementary bis-monodentate ligands, yielding structures such as rings (here presented). ${ }^{[80]}$ Studies involving supramolecular structures achieved by a "one pot" approach and systems driven by the principle of maximum site occupancy and self-assembly were performed, particularly based on the terpyridine and phenanthroline ligands with orthogonal coordination upon the addition of metal species. ${ }^{[81]}$ The inclusion of a trans-chelating ligand into this library enriches and contributes to expand new possibilities of systems, especially if we bring to the scene the application of DNA as a material for supramolecular nanoconstructs $^{[8,51]}$.

The idea of artificial DNA carrying ligands able to produce, through metal coordination, nanocontrucs, is known. But the potential of hybrids systems, containing different modifications able to bind selectively to different metal species in an orthogonal way to produce high-ordered systems, brings new potential to this field. Through dashing nanoconstructs will be able to produce switch and mimicking mechanisms with several possible applications. Nevertheless, initially it is necessary to study the possibility of orthogonal self-assembly between the ligands terpyridine, trans-chelating and phenanthroline ligands and metal species to confirm such potential applications. 


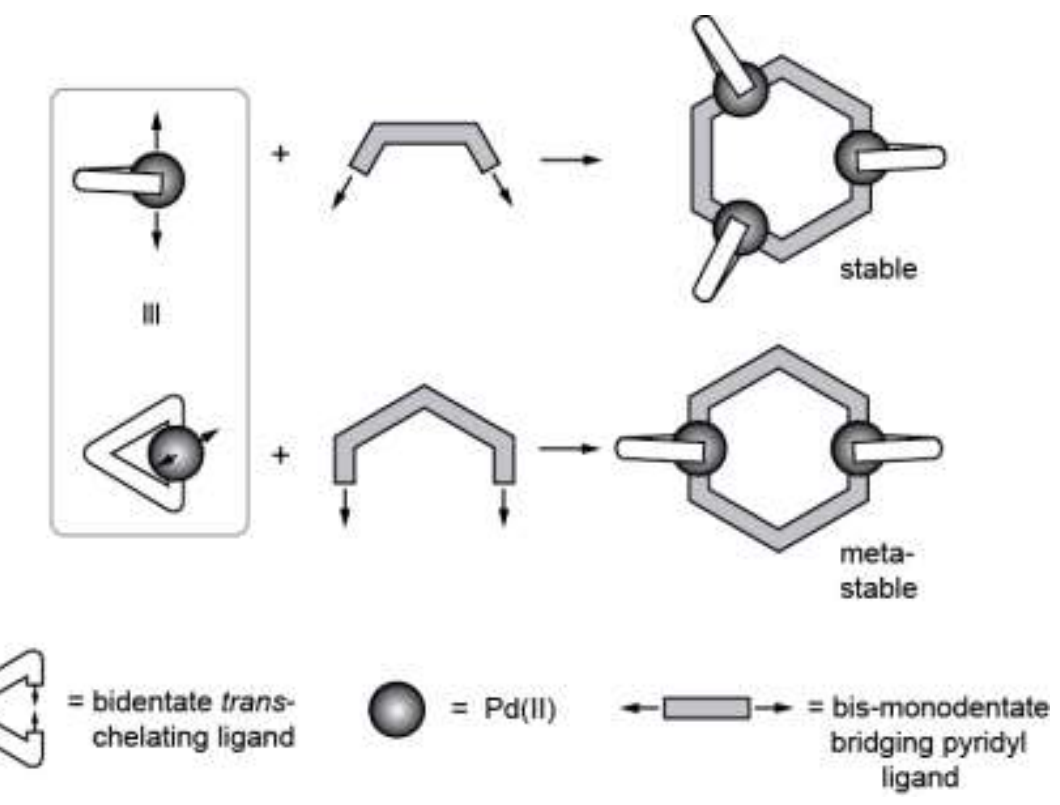

Figure 31. Scheme for the self-assembly of trans-chelated square-planar Pd(II) complex with bent ligands as linkers, producing rings of different sizes and thermodynamic stabilities. ${ }^{[80]}$

\subsubsection{Synthesis of monomeric complexes}

Synthetic details for ligands and complexes can be found in the experimental section 4.1. The monomeric complex $\left[\mathrm{Pd}(\mathbf{L 2}-\mathbf{2 c})(\mathrm{MeCN})_{2}\right]^{2+}$ demands for the ligand 1,2-bis(2-pyridyl-ethinyl)benzene (L2) the initial synthesis of a precursor complex, namely, $\left[\mathrm{Pd}\left(\mathbf{L 2}_{\mathbf{2}}\right) \mathrm{Cl}_{2}\right] .{ }^{[78]}$ The precursor is necessary because tries to obtain the desired monomer as a direct product upon addition of $\left[\mathrm{Pd}(\mathrm{MeCN})_{4}\right]\left(\mathrm{BF}_{4}\right)_{2}$, even with a stoichiometric amount, provides directly the dimer $\left[\mathrm{Pd}(\mathbf{L 2})_{2}\right]^{2+}$. This occur because the ligands $\mathbf{L} \mathbf{2}$ and $\mathbf{L} \mathbf{2} \mathbf{c}$ are more electron rich than the derivative $\mathbf{L} \mathbf{2 b}$ (see Figure 32). The ligand L2b contains on its backbone a group which draws the electron density away from the pyridyl rings causing a negative inductive effect. This makes it possible that under 1:1 equimolar addition of $\left[\mathrm{Pd}(\mathrm{MeCN})_{4}\right]\left(\mathrm{BF}_{4}\right)_{2}$, the direct formation of a monomer complex $\left[\mathbf{P d}(\mathbf{L} \mathbf{2 b})(\mathrm{MeCN})_{2}\right]^{2+}$ occurs. For ligand $\mathbf{L} \mathbf{2}$ and $\mathbf{L} \mathbf{2 c}$, the corresponding complex is prepared through the addition of 2 equimolar amounts of $\mathrm{AgBF}_{4}$ into $\left[\mathrm{Pd}(\mathbf{L 2 - 2 c}) \mathrm{Cl}_{2}\right]$, producing $\mathrm{AgCl}$ (that is precipitated and filtered) and $\left[\mathrm{Pd}(\mathbf{L 2}-\mathbf{2 c})(\mathrm{MeCN})_{2}\right]^{2+}$. All the complexes were prepared in $\mathrm{CD}_{3} \mathrm{CN}$ allowing their formation to be characterized by ${ }^{1} \mathrm{H}$ NMR spectroscopy. The importance of such a monomer complex is due to its ability to act as a building block, where it is possible for a further replacement of the two acetonitrile groups coordinated to $\{\mathbf{P d}(\mathbf{L} \mathbf{2}-\mathbf{2 b}$ 2c)\} by any other bis-monodentate ligands. This will create a bridge between these fragments and produce highly ordered heteroleptic structures such as rings or promote the reassembly to other thermodinamically stable structures (it depends on their coordination angle and properties) in accordance to the principle of maximum site of occupancy. ${ }^{[77]}$ 
a)

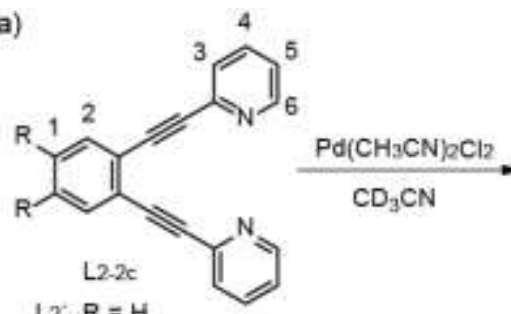

L2: $\mathrm{R}=\mathrm{H}$

$L_{20}: R=$ OTBDMS

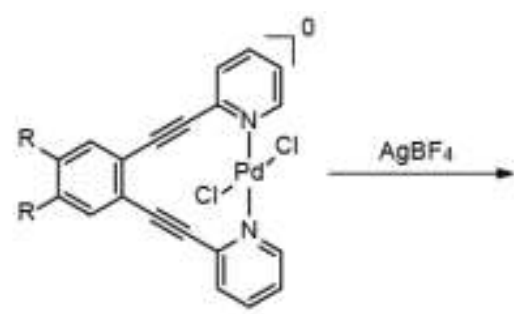

$[\mathrm{Pd}(\mathrm{Lz}-2 \mathrm{C})(\mathrm{Cl}) 2]$

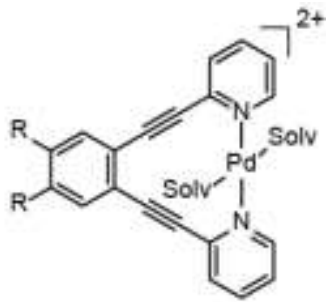

$\left[\mathrm{Pd}(\mathrm{L} 2-2 \mathrm{r})\left(\mathrm{CD}_{3} \mathrm{CN}\right) 2\right]^{2+}$

b)
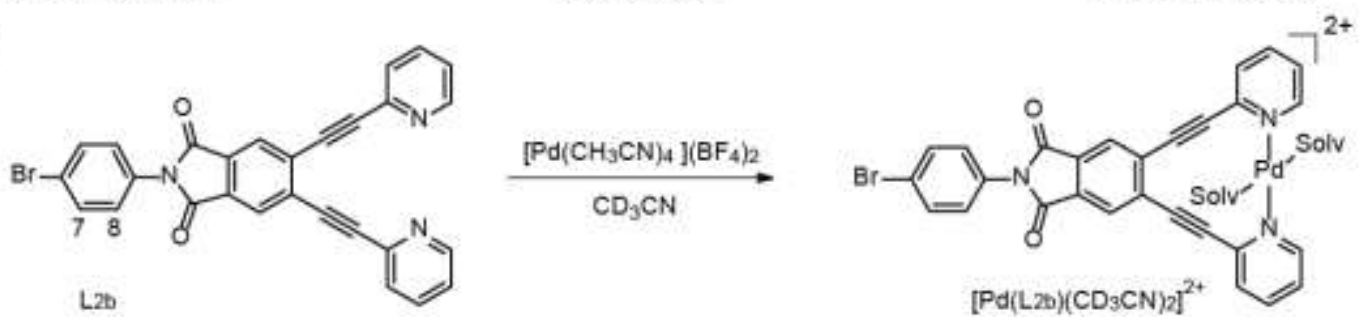

Figure 32. Reactional scheme for the monomeric complexes synthesized. a) Scheme for $\mathbf{L} \mathbf{2}$ and L2c, electron rich derivatives which need precursor complex $\left[\mathrm{Pd}(\mathbf{L 2}-\mathbf{2 c}) \mathrm{Cl}_{2}\right]$ formation and b) direct formation of $\left[\mathrm{Pd}(\mathrm{L} \mathbf{2 b})(\mathrm{MeCN})_{2}\right]^{2+}$ because of its electron deficiency.

The Figure 33 shows the ${ }^{1} \mathrm{H}$ NMR spectra for the ligand $\mathbf{L} 2$ and the respective spectra for its monomer and dimer complex, $\left[\mathrm{Pd}(\mathbf{L} 2)(\mathrm{MeCN})_{2}\right]^{2+}$ and $\left[\mathrm{Pd}(\mathbf{L 2})_{2}\right]^{2+}$, respectively. The spectra for the derivative $\mathbf{L} \mathbf{2 b}$ and its correspondent monomeric complex $\left[\mathbf{P d}(\mathbf{L} \mathbf{2 b})(\mathrm{MeCN})_{2}\right]^{2+}$ are also shown. It is possible to clearly distinguish between monomers and dimers via their different proton shifts. For all complexes formed a characteristic and significant downfield shift for the protons of the ligand was observed, especially for proton 6 , which is the neighbour proton to the atom $\mathrm{N}$ in the pyridine rings, that coordinates with $\mathrm{Pd}(\mathrm{II})$.

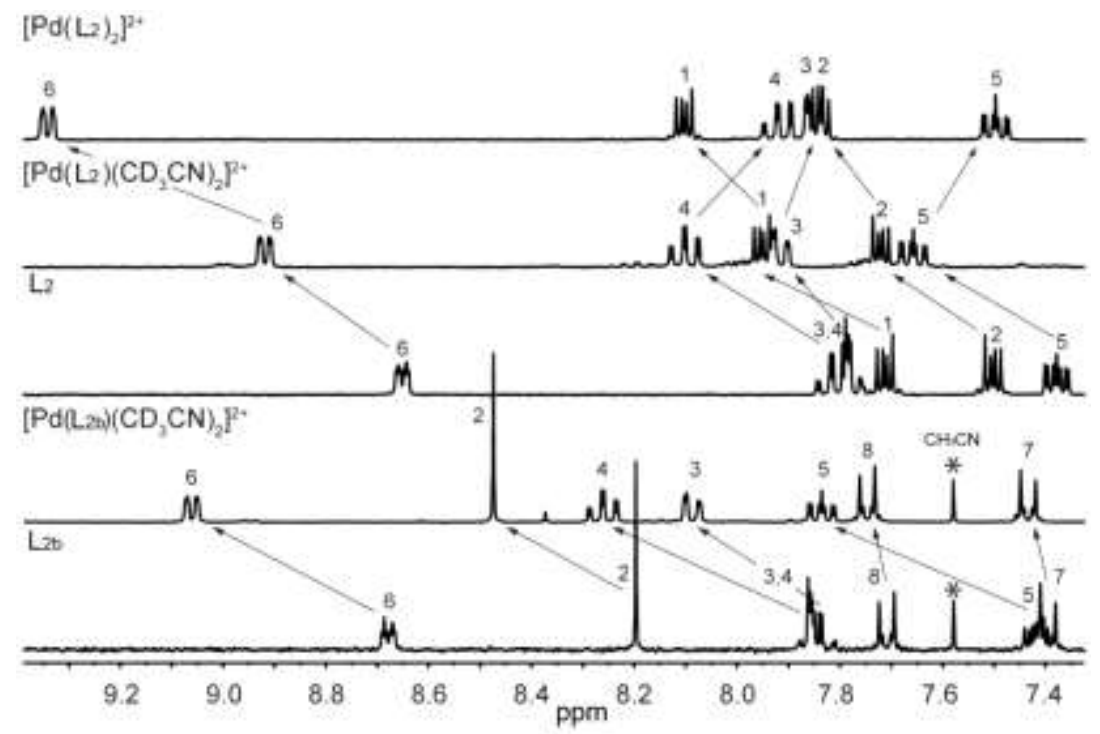

Figure 33. ${ }^{1} \mathrm{H}$ NMR spectra for ligand $\mathbf{L} \mathbf{2}$ and $\mathbf{L} \mathbf{2} \mathbf{b}$, and their respectives monomer complexes with $\mathrm{Pd}(\mathrm{II})$ and $\mathrm{CD}_{3} \mathrm{CN}$ and the dimer complex of $\mathbf{L} \mathbf{2}$. 


\subsubsection{Synthesis of the rings}

The formation of 2- and 3- membered rings containing $\{\mathrm{Pd}(\mathbf{L} \mathbf{2}-\mathbf{2 b}-\mathbf{2 c})\}$ units and their respective kinetic and thermodinamic properties depend on the type of ligand used to replace the $\mathrm{CD}_{3} \mathrm{CN}$ at the coordination site in monomeric complexes. The bis-monodentate ligands chosen will bridge the $\mathrm{Pd}(\mathrm{II})$ centres and the angle between the bonds drives the number of $\{\mathrm{Pd}(\mathbf{L} \mathbf{2}-\mathbf{2} \mathbf{b}-\mathbf{2 c})\}$ on the ring assembly (Figure 34).

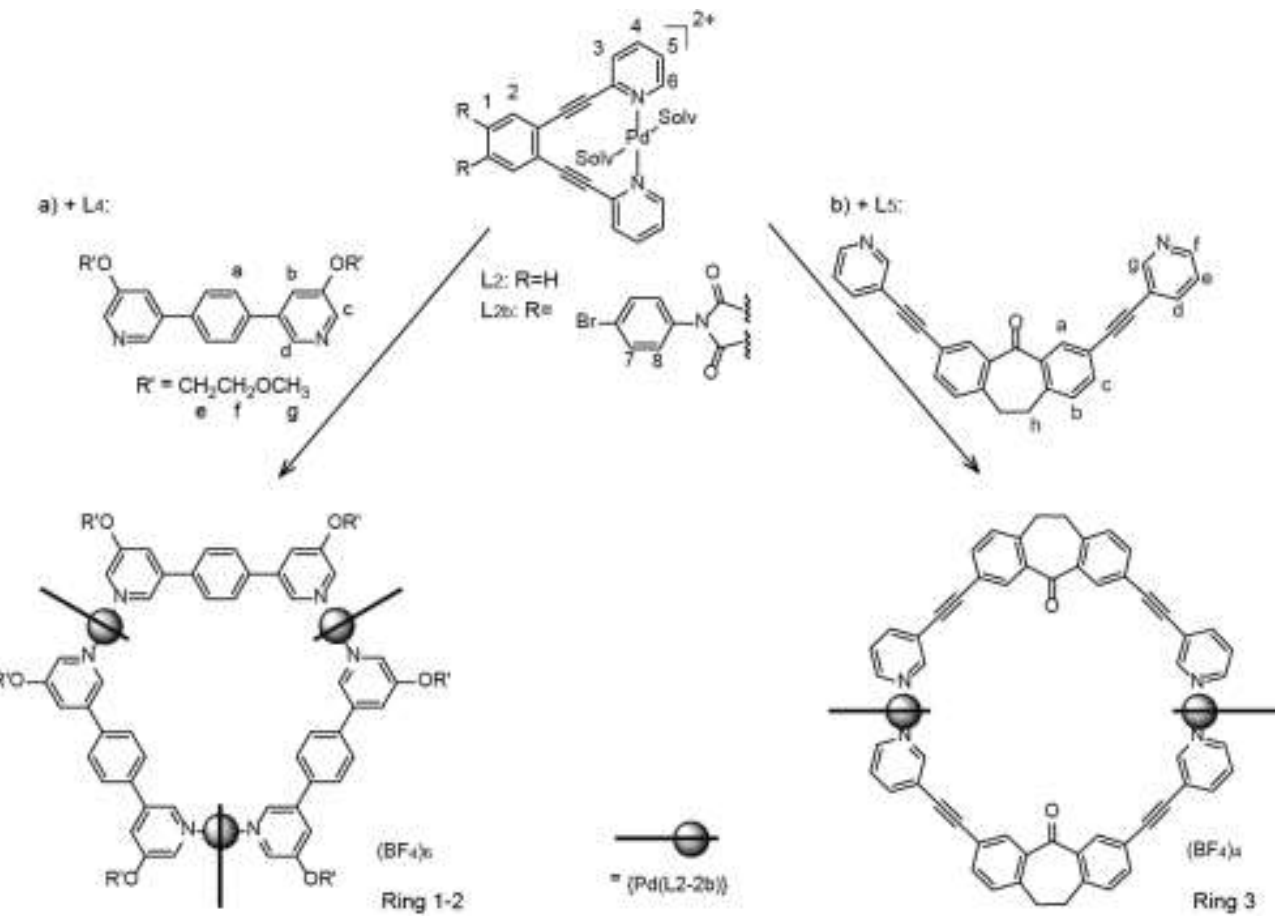

Figure 34. Scheme for assembly of 2-and 3-membered rings from the respective ligands and Pd(II) complexes.

Ligand L4 is linear and has the oxygen atom, responsible for the binding link, attached to the aromatic ring in meta position, which gives an orientation with an angle around $120^{\circ}$, allowing for the formation of a 3 -membered ring. Ligand $\mathbf{L} 5$ has a banana shape ${ }^{[82]}$ where the binding is through the pyridine arms placed on both sides of the bent backbone, allowing for the formation of a 2-membered ring. The assembly of the rings relies on a simple stoichiometric addition of ligand $\mathbf{L} \mathbf{4}$ or $\mathbf{L 5}$, at room temperature, into the respective monomer complex solution.

The formation of the rings could be monitored by NMR spectroscopy (Figure 35), where quantitative formation can be observed for all cases. The formation of the 3-membered rings with L2 and L2c ligands suggestes a more stable complex than the correspondent one with ligand L2b (the ring dissociates completelly after a time around $12 \mathrm{~h}$ at room temperature). This is probably related to its electron deficiency with respect to ligands $\mathbf{L} \mathbf{2}$ and $\mathbf{L} 2 \mathbf{2}$. Upon 
formation, for all 3-membered rings obtained, the downfield shift became stronger, especially for proton 6 .

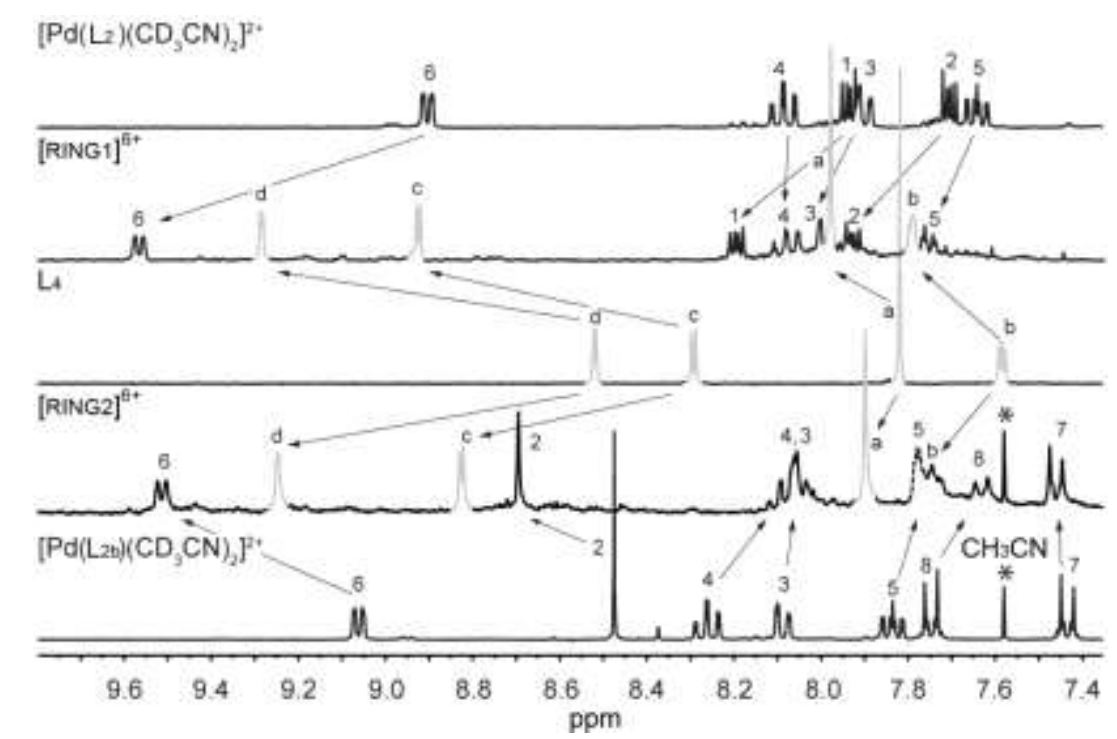

Figure $35 .{ }^{1} \mathrm{H}$ NMR spectra for 2- and 3- membered rings formed.

ESI mass spectrometry of the 3-memberend ring with L2 (Figure 36) revealed several peaks with isotopic pattern matching with the calculated pattern for relevant ring structure.

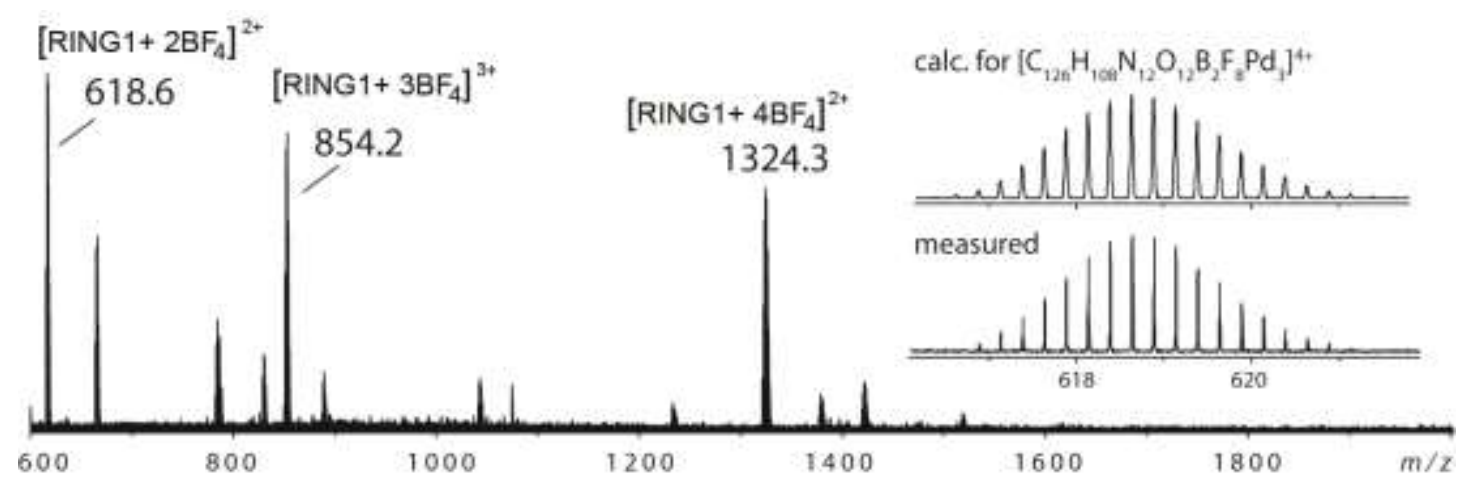

Figure 36. ESI mass spectra for RING1 and respective isotopic pattern, calculated and measured.

The ${ }^{1} \mathrm{H}$ NMR stacking of the assembly of the 2-membered ring (Figure 38 ) is coherent for the data set obtained for the 3-membered rings, with downfield shift of all protons after complexation that becomes even more expressive upon the ring formation. The protons corresponding to the backbone of bridging ligand $\mathbf{L} \mathbf{5}$ remains virtually unshifted indicating that these protons are not affected by ring formation. Another interesting fact related to the ligand $\mathbf{L} \mathbf{5}$ is related to its shape. In contrast to $\mathbf{L} \mathbf{4}$ which has an angle around $120^{\circ}$, the banana shape 
ligand L5 allows an assembly for higher-ordered structures, indicating a more thermodynamically stable complex structure as the product.

The 2-membered ring is initially obtained, as a kinetic product (meta-stable state) and later it was possible to observe by ${ }^{1} \mathrm{H}$ NMR (Figure 38) and ESI-MS (Figure 39), after leaving the ring sample at room temperature after several days, that the structure converts to a mixture of a interpenetrated double-cage, $\left[2 \mathrm{Cl}+\mathrm{BF}_{4} @ \mathrm{Pd}_{4} \mathrm{~L}_{8}\right]\left(\mathrm{BF}_{4}\right)$ and dimer $\left[\mathrm{Pd}\left(\mathrm{L2}_{2}\right)_{2}\right]\left(\mathrm{BF}_{4}\right)_{2}$. This double cage is already known and published by colleagues (Figure 37 ). ${ }^{[83,84]}$ It is also known that the interpenetrated double cage formed is dependent on the presence of $2 \mathrm{Cl}^{-}$, which located the three inner pockets of the cage. The presence of chloride ions in solution is probably due to traces of $\mathrm{AgCl}$ stemming the conversion of $\left[\mathrm{Pd}(\mathbf{L} 2) \mathrm{Cl}_{2}\right]^{2+}$ to $\left[\mathrm{Pd}(\mathbf{L 2})(\mathrm{MeCN})_{2}\right]^{2+}$.

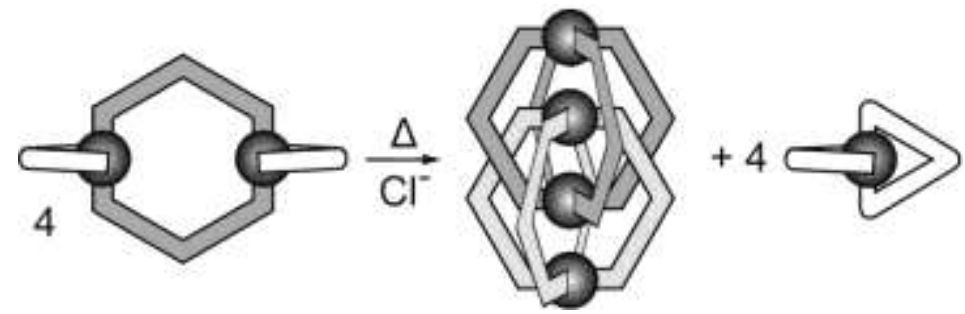

Figure 37. Scheme for conversion of RING3 into the interpenetrated double-cage + dimer.

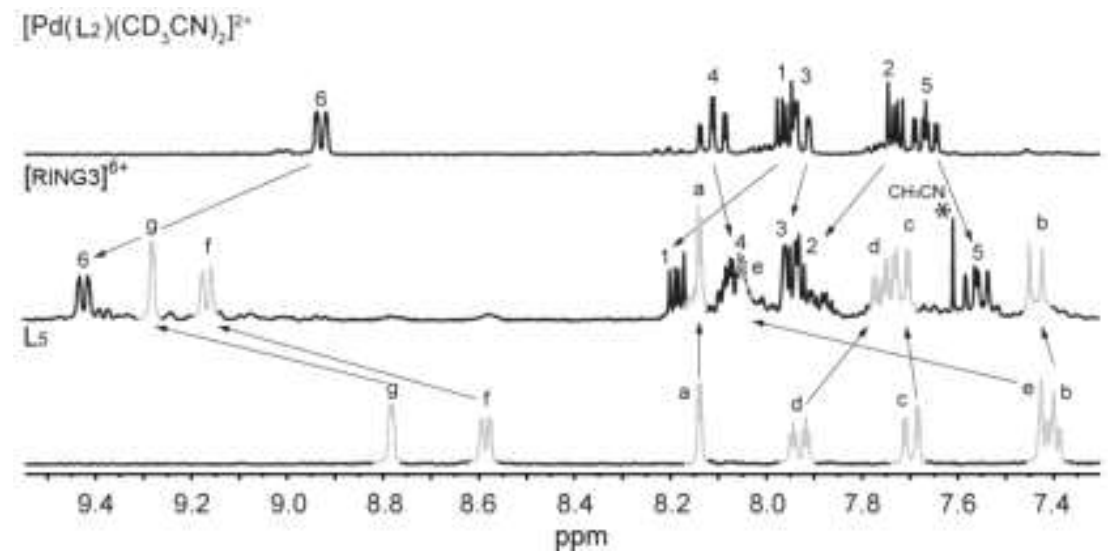

Figure 38. ${ }^{1} \mathrm{H}$ NMR spectra indicating the convertion of the 2-memberd ring (RING3) to the interpenetrated double-cage $\left[2 \mathrm{Cl}+\mathrm{BF}_{4} @ \mathrm{Pd}_{4} \mathrm{L5}_{8}\right]\left(\mathrm{BF}_{4}\right)$ and $\operatorname{dimer}\left[\mathrm{Pd}(\mathbf{L 2})_{2}\right]\left(\mathrm{BF}_{4}\right)_{2}$. 
a)

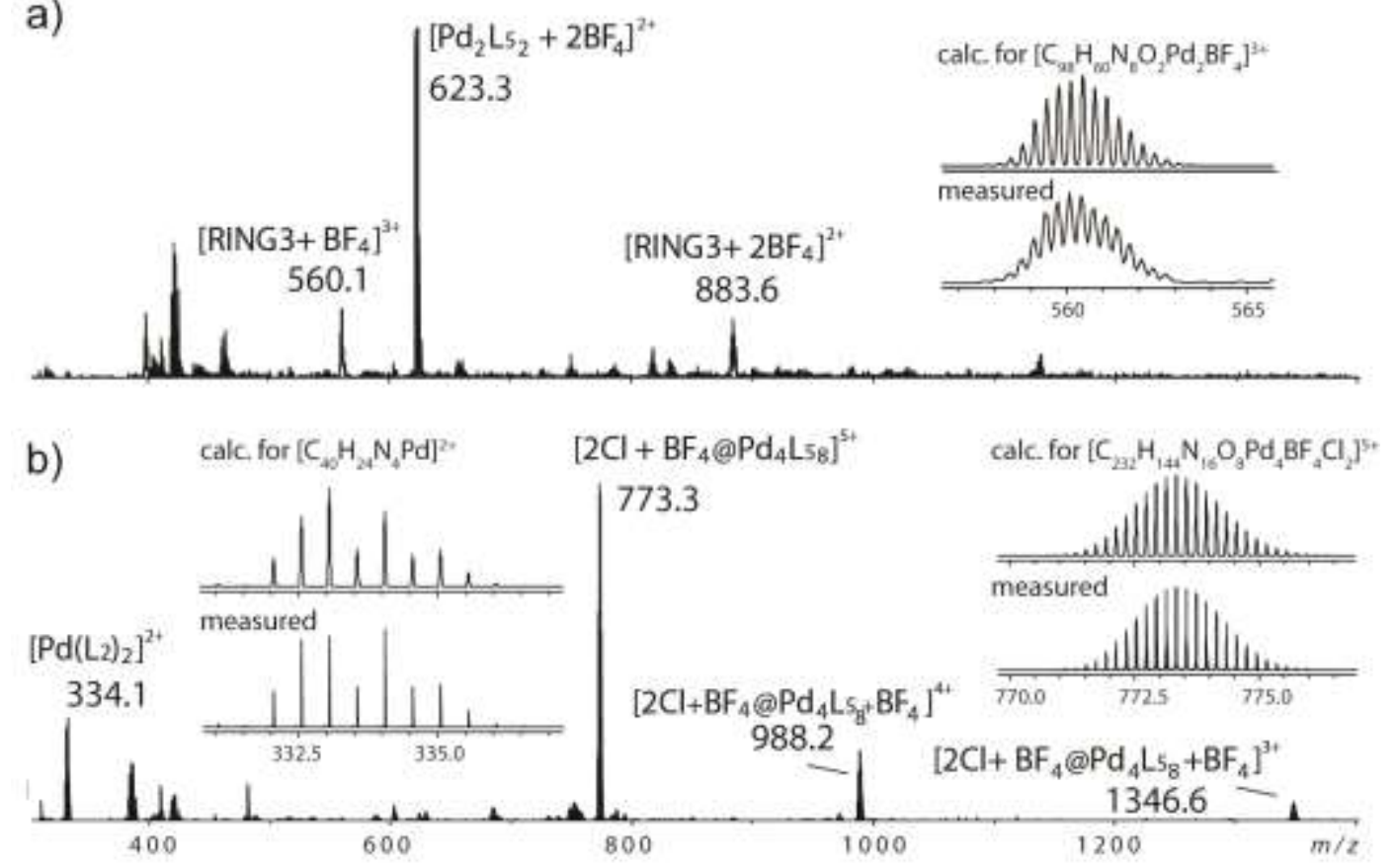

Figure 39. ESI spectra for RING3 and its convertion to the double cage after several days at room temperature.

More details about the rings were determinated by modeling (semiempiric PM6 level of theory, Gaussian '09) and can be found in appendix (Figure 97). The calculations indicate a $C_{3}$ symmetry for 3-membered rings, where a side-view shows that the ring is totally flat with an ideal square-planar coordination around the Pd centres and an unconstrained character for the bridging ligand $\mathrm{L} 4$. The angle between the $3 \mathrm{Pd}$ centres is $60^{\circ}$ and the distance between the Pd units is $12.3 \AA$. On the 2-membered ring the plane for the trans-chelating units is perpendicular to the bridging ligand $L 5$, which indicates a $C_{2}$ symmetry while the global ring had a $D_{2}$ symmetry.

\subsubsection{Study of orthogonal self-assembly}

The application of heteroleptic systems with controlled complex formation upon addition of different metal species to produce metal-nanoconstructs opens possibilities in supramolecular chemistry to develop high-ordered systems with selectivity able to mimick mechanisms with several applications. The insertion of trans-chelating ligand to the library together with terpyridine and phenanthroline enrich the extention of possibilities, but initially the orthogonal self-assembly between the primary systems must to be tested for further applications for nanoconstructs. The ligands 4`-(4-bromo-phenyl)-2-2`-6’-2"'-terpyridine (L1), 1,2-bis(2-pyridylethinyl)-benzene (L2) and phenanthroline (L3) were combined with salts of $\mathrm{Fe}(\mathrm{II}), \mathrm{Cu}(\mathrm{I})$ and $\mathrm{Pd}(\mathrm{II})$ in acetonitrile solutions with the goal to produce a library of orthogonal self-assemblies 
and obtain the complexes $\left[\mathrm{Fe}(\mathbf{L} 1)_{2}\right]^{2+},\left[\mathrm{Pd}(\mathbf{L} 2)_{2}\right]^{2+},\left[\mathrm{Cu}(\mathbf{L} \mathbf{3})_{2}\right]^{1+}$ without by-products interferences or competition between the species. The key to obtain successful combinations is the sequence taken in adding the metal species and allowing heating between each addition to accelerate the full dimerization to obtain all the desired dimeric complexes.
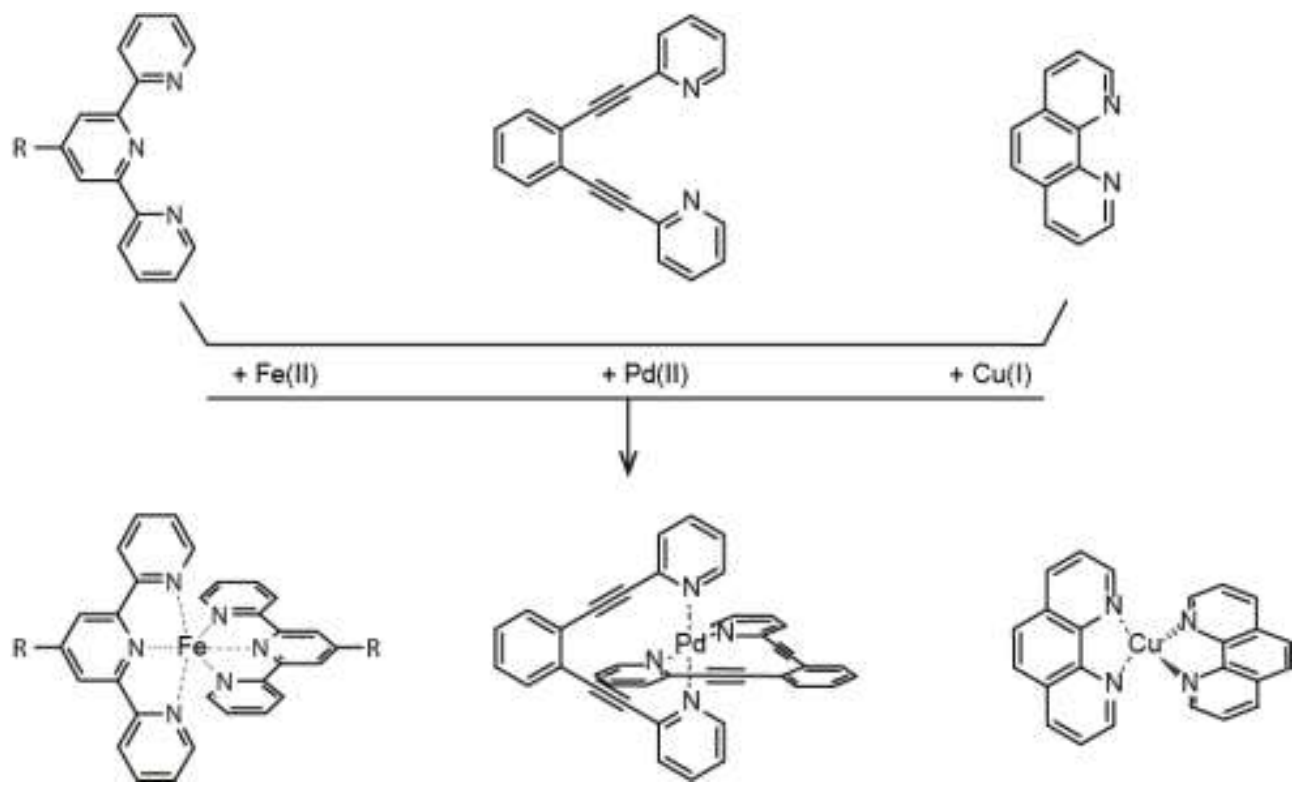

Figure 40. Scheme for the desired dimer complexes, $\left[\mathrm{Fe}(\mathbf{L} \mathbf{1})_{2}\right]^{2+},\left[\mathrm{Pd}(\mathbf{L} \mathbf{2})_{2}\right]^{2+},\left[\mathrm{Cu}(\mathbf{L} \mathbf{3})_{2}\right]^{1+}$, respectively. In ligand $\mathbf{L} 1, \mathrm{R}=-\mathrm{BrC}_{6} \mathrm{H}_{4}$.

By ${ }^{1} \mathrm{H}$ NMR it was possible to follow the formation of all complexes and study the competition between them based on titrations with several different addition sequences of components. Between the pairs of complexes, it was possible to observe the formation of $\left[\mathrm{Fe}(\mathbf{L} \mathbf{1})_{2}\right]^{2+}$ and $\left[\mathrm{Pd}(\mathbf{L 2})_{2}\right]^{2+}$ in the same solution as well as the mutual formation of $\left[\mathrm{Cu}(\mathrm{L} \mathbf{3})_{2}\right]^{1+}$ and $\left[\mathrm{Pd}(\mathbf{L 2})_{2}\right]^{2+}$. Both cases are possible due to the high selectivity of the trans-chelating ligand for Pd(II). Even very small amounts of complexes of $\mathbf{L} 2$ and $\mathrm{Fe}(\mathrm{II})$ or $\mathrm{Cu}(\mathrm{I})$ could be completely reverted after heating the mixture, thus shifting the equilibrium to a full formation of $\left[\mathrm{Pd}(\mathbf{L} 2)_{2}\right]^{2+}$.

The initial formation of $\left[\mathrm{Cu}(\mathrm{L} 3)_{2}\right]^{+}$followed by the addition of $\mathrm{Fe}(\mathrm{II})$ causes a competition between the metals; some $\left[\mathrm{Fe}(\mathbf{L} 3)_{2}\right)^{2+}$ will always form. The further addition of ligand with heating does not reverse the equilibrium to a complete formation of $\left[\mathrm{Cu}(\mathbf{L} 3)_{2}\right]^{+}$and $\left[\mathrm{Fe}(\mathbf{L} \mathbf{1})_{2}\right)^{2+}$, there will always have some $\left[\mathrm{Fe}\left(\mathbf{L}_{3}\right)_{2}\right]^{2+}$ formed. To avoid it, the iron(II) must be fully coordinated with $\mathbf{L} 1$ before the addition of $\mathrm{Cu}(\mathrm{I})$, and then the addition of the free phenanthroline. This latter sequence gives the desired outcome.

When combining a solution of $\left[\mathrm{Cu}(\mathbf{L} 3)_{2}\right]^{+}$and $\left[\mathrm{Fe}(\mathbf{L} 1)_{2}\right]^{2+}$, the further addition of trans-ligand $\mathbf{L} 2$ makes the ${ }^{1} \mathrm{H}$ NMR peaks too broad and so it was not possible to conclude what happens. 
Further addition of $\mathrm{Pd}(\mathrm{II})$ and heating to form $\left[\mathrm{Pd}(\mathbf{L} 2)_{2}\right]^{2+}$ was expected to restore good conditions to visualize ${ }^{1} \mathrm{H} N \mathrm{NR}$, but it did not work and the products formed could not be followed by ${ }^{1} \mathrm{H}$ NMR. The solution to obtain a mixture of all three desired consists to initially mix all the 3 ligands, $\mathbf{L} \mathbf{1}, \mathbf{L} \mathbf{2}$ and $\mathbf{L} \mathbf{3}$ together. Then Fe(II) is added first, to coordinate with $\mathbf{L} \mathbf{1}$ present in the solution. After complete formation of $\left[\mathrm{Fe}(\mathbf{L} \mathbf{1})_{2}\right)^{2+}$ in presence of free $\mathbf{L} \mathbf{2}$ and $\mathbf{L} \mathbf{3}$, $\mathrm{Pd}(\mathrm{II})$ is added which selectively form the $\left[\mathrm{Pd}\left(\mathrm{L}_{2}\right)_{2}\right]^{2+}$ complex after heating. Finally, the $\mathrm{Cu}(\mathrm{I})$ was added to the mixture still containing free $\mathrm{L} 3$ to form $\left[\mathrm{Cu}(\mathrm{L} \mathbf{3})_{2}\right]^{+} .{ }^{1} \mathrm{H} N M R$ and ESI-MS results confirm the formation of the 3 complexes in the same solution, as shown below (Figure 41 and 42 respectivelly).

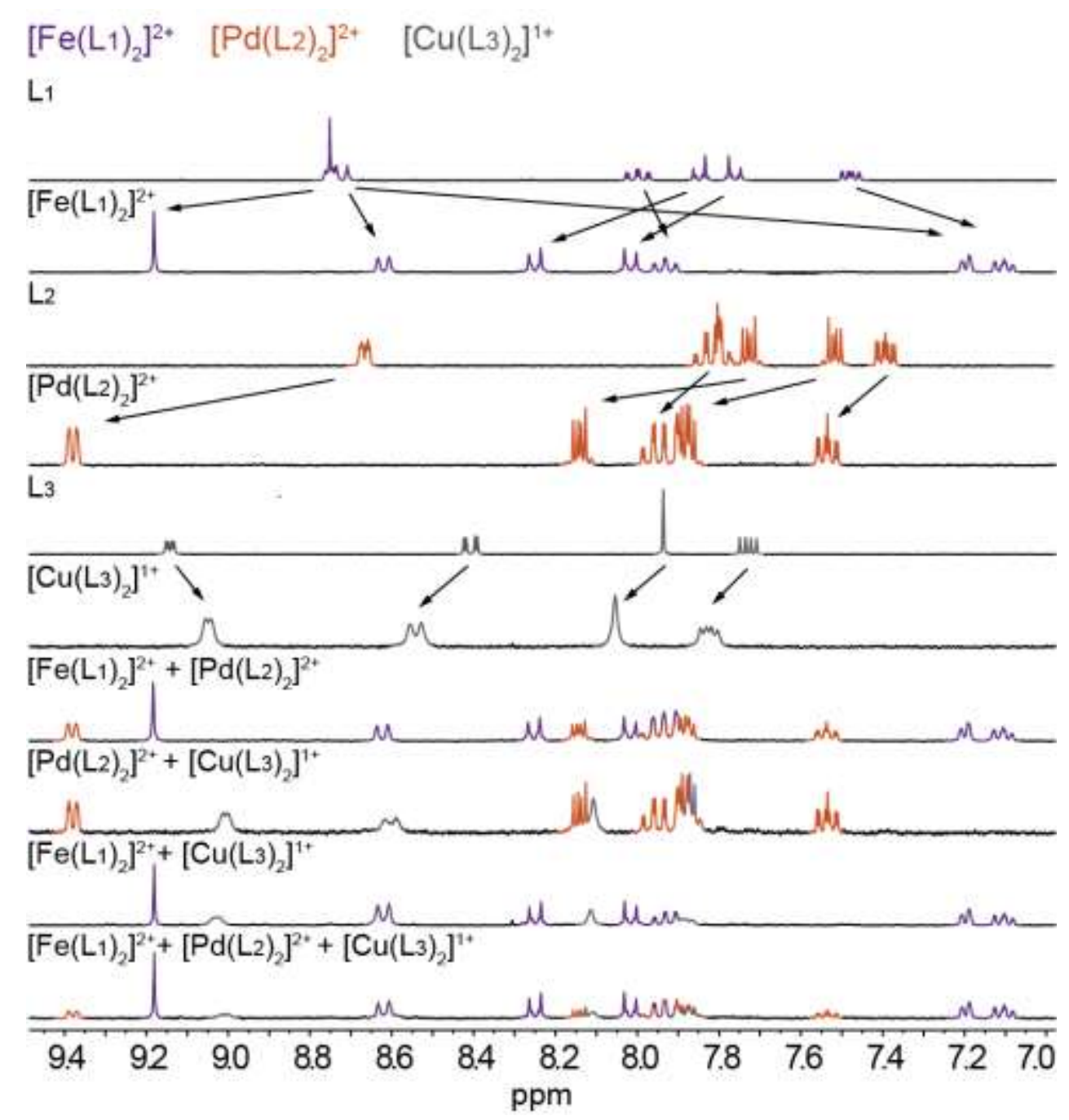

Figure $41 .{ }^{1} \mathrm{H}$ NMR for dimer complexes in $\mathrm{CD}_{3} \mathrm{CN}$. 


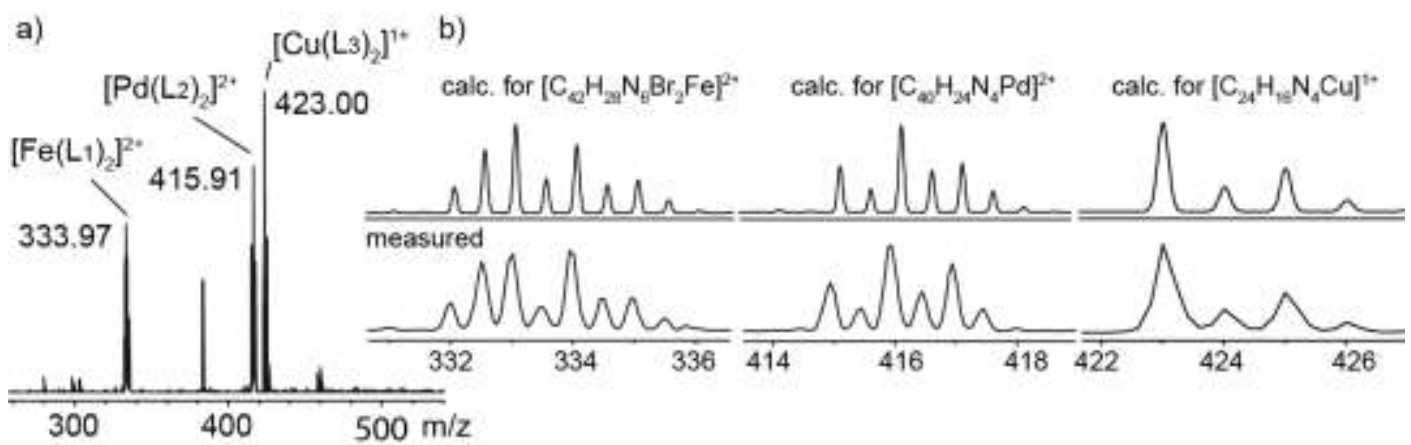

Figure 42. a) ESI-MS for dimer complexes together under same solution, b) Isotopic pattern for the calculated and measured peaks of each dimer. 


\subsection{Aims of the project (part 2) - Metal Base Pairing based on trans-chelating ligand}

In the recent years, the field of synthetic modified DNA has emerged strongly, providing new possibilities and potential to the research area even beyond biological applications. The use of DNA as a material for supramolecular chemistry is related to the fact that its secondary structures is easy to manipulate. DNA can be prepared easily through solid phase synthesis (SPS), where small oligonucleotides can behave like short rigid sticks and the chemical modification through artificial nucleosides brings new functionalities. The metal-base pairing (MBP) is a concept developed where the Watson-Crick base pairing, based on hydrogen bonding in natural DNA double helix, is replaced by metal coordination between the artificial ligands incorporated into the oligonucleotide strands (one is placed in each strand in a way to face each other in double helix formation). ${ }^{[50]}$ Such systems have shown be successful to open new functionalities with new and interesting properties, in most of the cases, despite not contributing to the base stacking of the system, metal coordination provides extra stabilty through the complex formation. ${ }^{[8]}$

The main goal of this part $\mathbf{2}$ of the project is to design a new type of ligand to be incorporated into a DNA sequence and be able to coordinate with $\mathrm{M}$ (II) species to form a new type of metalbase pair. The ligand has to be designed in a way to fit and follow the helical shape structure and base stacking of the double helix, without causing distortions, or at least, as little as possible. The first MBP developed were designed and created by Shionoya ${ }^{[85]}$ and Schultz ${ }^{[54]}$ and since then, several other systems with interesting applications have been developed.

\subsubsection{Synthesis of the nucleoside}

It was the pursuit of a design consisting of a metal-base pairing based on a trans-chelating ligand with pyridyl arms, able to bind with $\mathrm{M}(\mathrm{II})$ species such as $\mathrm{Pd}(\mathrm{II})$ to give a square-planar complex. It enriches possibilities of intraduplex applications and DNA nanoconstructs. This results from its potential for hybrids systems related to orthogonality with other artificial nucleobases based in terpyridine and phenanthroline groups and complexes formed with $M(I I)$ and $\mathrm{M}(\mathrm{I})$ ions.

The building block ligand and its respective phosphoramidite incorporated into DNA were synthesized starting from 2-bromo-5-hydroxy-pyridine, which is attached a hydroxyl- 
terminated linker. The next step is an alkynylation by Sonogashira cross-coupling which is also used to attach it twice to the 1,2-diiodobenzene, yielding the trans-chelating scaffold $\mathbf{4}$ that is later protected in one of the sides with a 4,4'-dimethoxytrityl group (DMT) and the other side is turned into a phosphoramidite yielding the compound 6 that is applied for solid phase DNA synthesis. More synthetic details and characterization for all steps can be found in the experimental part.

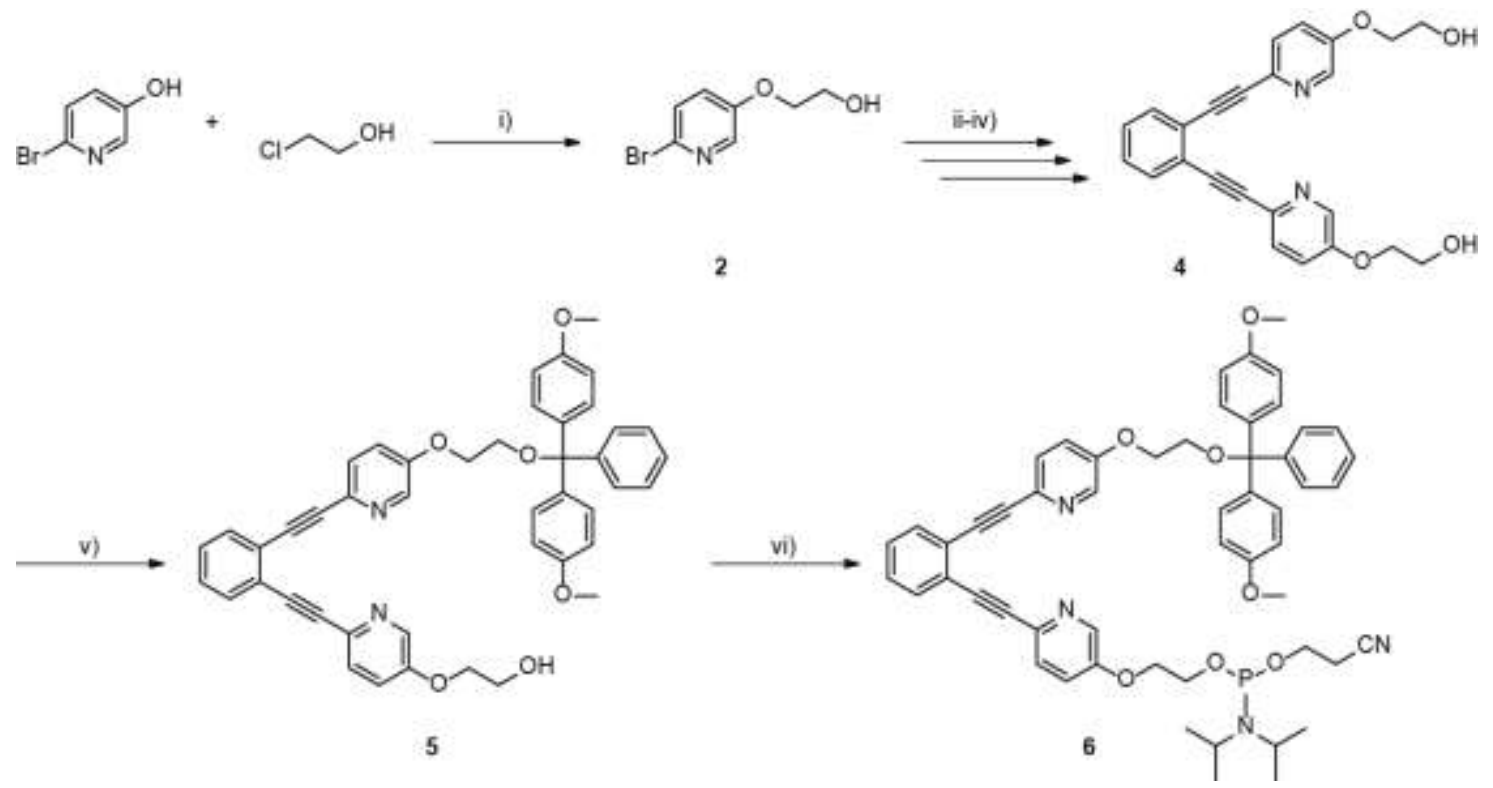

Figure 43. Scheme of synthetic route for trans-chelating ligand 5 and phosphoramidite $\mathbf{6}$. i) $\mathrm{K}_{2} \mathrm{CO}_{3}$, MeCN, reflux 18h; ii) TMS-acetylene, THF, Cul, $\mathrm{PdCl}_{2}\left(\mathrm{PPh}_{3}\right)_{2}, \mathrm{Et}_{3} \mathrm{~N}$, r.t., 5h; iii) $\mathrm{K}_{2} \mathrm{CO}_{3}$, $\mathrm{MeOH}, 8$ h; iv) 1,2-diiodobenzene, $\mathrm{Cul}, \mathrm{PdCl}_{2}\left(\mathrm{PPh}_{3}\right)_{2}$, dry $\mathrm{THF}, \mathrm{Et}_{3} \mathrm{~N}$, r.t., 8h; v) DMT-Cl, py, DMAP, 24h; vi) ( $\left.\operatorname{PPr}_{2} \mathrm{~N}\right)\left(\mathrm{OCH}_{2} \mathrm{CH}_{2} \mathrm{CN}\right) \mathrm{PCl}, \mathrm{NEt}(\mathrm{iPr})_{2}, \mathrm{THF}, 2 \mathrm{~h}$.

\subsubsection{Incorporation into oligonucleotides}

The synthesis of two complementary oligonucleotide sequences containing in each strand one trans-chelating ligand incorporated, will produce a DNA double helix that presents a "gap" in the structure with no Watson-Crick base pairing. ${ }^{[37]}$ The potential formation of further metalbase pairing after addition of $M(I I)$ happens due to the square-planar complex formed, providing a higher stability to the system and closing such break on the helix structure. The bond between $M(I I)$ and the two pyridyl-arms, together with the terminal linkers based on hydroxyl groups attached on both sides of the ligand, provides a design suitable to twist and follow the helical shape of the double helix, where the square-planar complex produced presents a propeller shape ${ }^{[78]}$ not presenting strong distortions on the stability of the system, as can be observed on the following thermal stability results. 


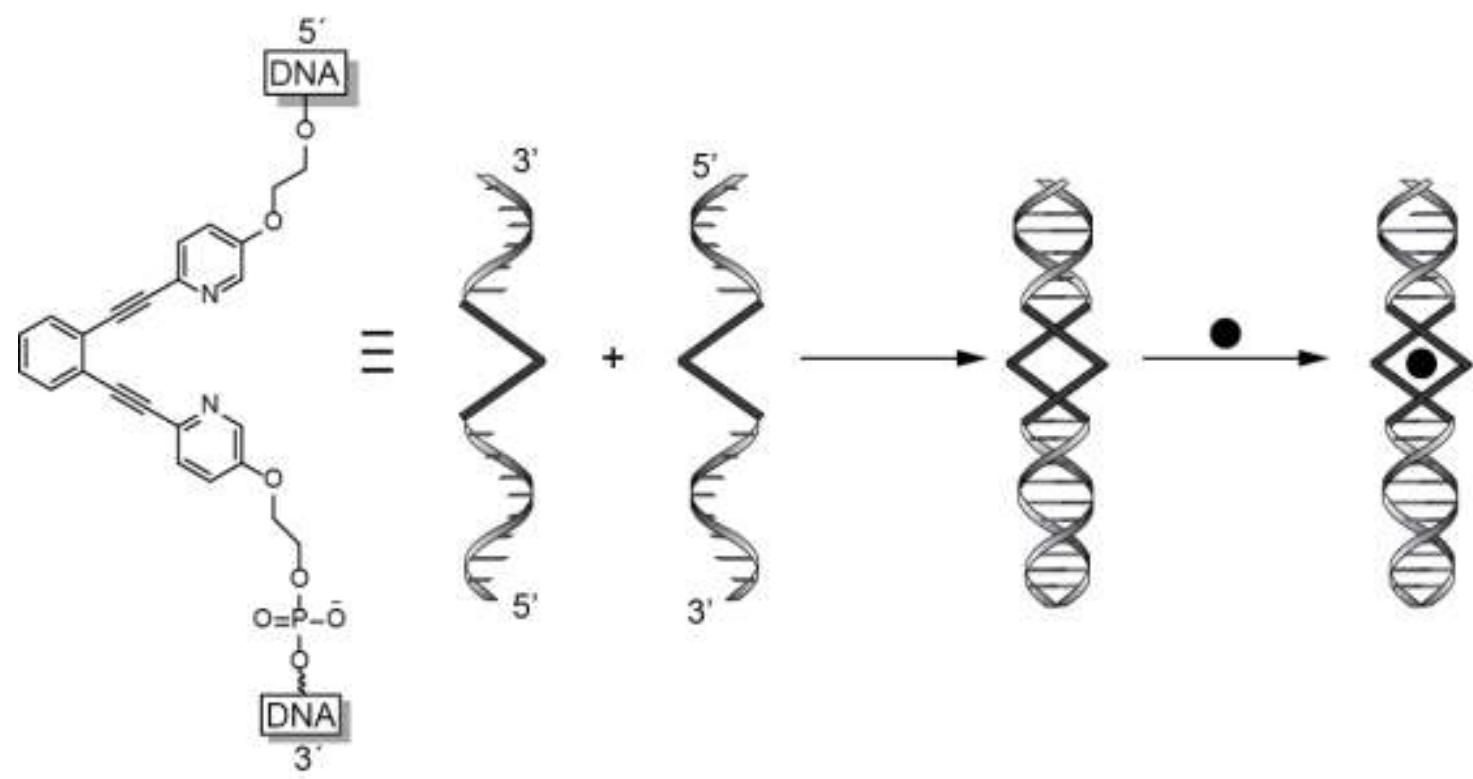

Figure 44. Scheme for trans-chelating ligand incorporated into DNA and respective complementary single strands and $d s$-DNA formed and its respective metal-base pairing.

There is a chirality transfer from DNA onto ligands incorporated and the square-planar complex formed inside the $d s$-DNA may have its stereochemistry driven by the DNA helix. ${ }^{[86]}$ The complex can form two stereoisomers, a) in the same direction of the twist for B-DNA structure, which means a right-handed structure or b) adopt the opposite direction. Theorically both possibilities are susceptible, but to assign which isomer would be favorable it is necessary a deeper theorical analysis with modeling studies for both isomers and their respectives energies.

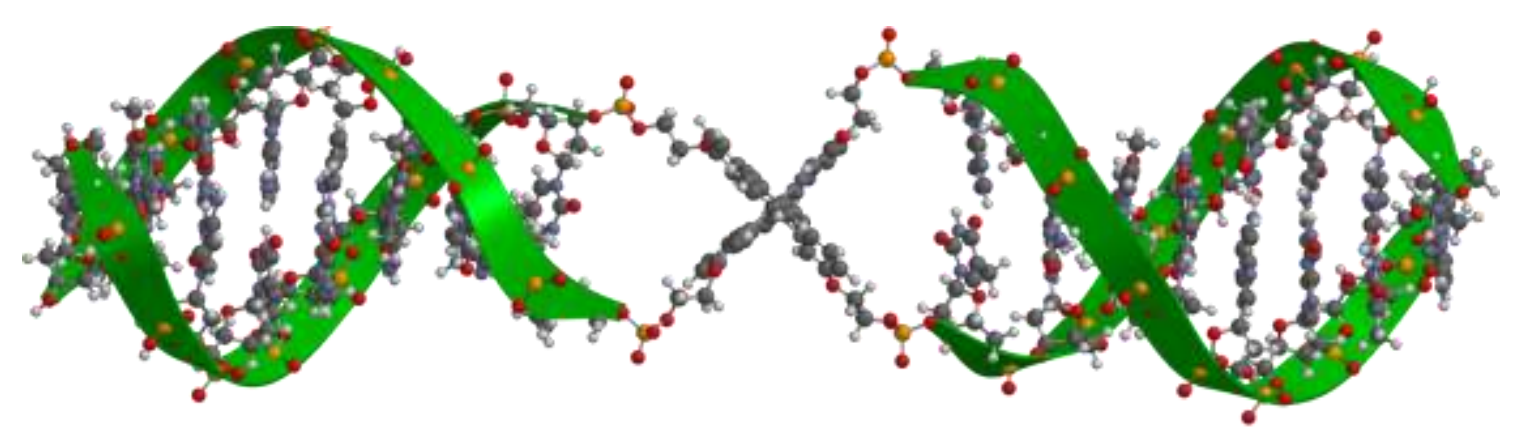

Figure 45. Rough MMFF model made with the Spartan software for modified $d s$-DNA containing MBP based on trans-chelating ligand and M(II). ${ }^{[87]}$ 
a) DNA syntheses

The DNA oligonucleotides for part 2 were synthesized with a K\&A Synthesizer $\mathrm{H}-8$, on a $1 \mu$ mol scale. All the solutions required for SPS synthesis were prepared on the same day as the DNA syntheses. The protected phosphoramidites were purchased from Sigma aldrich and diluted in dry acetonitrile to produce $0.1 \mathrm{M}$ solutions. Previous to each synthesis, an unmodified analogue strand was prepared to make sure that the DNA synthesizer and all reagents were in good conditions. The DNA strands were synthesized on a DMT-ON mode to facilitate further purification by RP-HPLC. The oligonucleotide synthesis was monitored by UV/Vis absorption, correspondent to DMT cleavage (during detritylation step) on each base coupling and in yields superior than $80 \%$. The cation of $4,4^{\prime}$-dimethoxytrityl has a strong red color and can be detected by UV/Vis at $500 \mathrm{~nm}$. Modified phosphoramidite was prepared as $0.1 \mathrm{M}$ and $0.05 \mathrm{M}$ solution with dry acetonitrile. No significant change on the coupling efficiency was observed by variation of the concentration.

The synthesized strands were 5'- TAC AAC AXT AAT GTG -3' and 5'- CAC ATT AXT GTT GTA -3', denominated as $\mathbf{s} \mathbf{1}$ and $\mathbf{s} \mathbf{2}$, respectivelly.

b) Deprotection of the oligonucleotides and purification:

The cleavage of the synthesized strands from the solid support and deprotections of the bases were performed with a $3 \mathrm{M}$ ammonium hydroxide solution 50:50 water/methanol ( 1 $\mathrm{mL})$ and left for 8 hours at $55^{\circ} \mathrm{C}$ in a thermomixer. As a standard, higher concentrated ammonium solution is normally used, but based on preliminary synthesis made for the next oligonucleotides (part 3) milder conditions were adopted, which showed to be efficient without any observed decomposition of the incorporated ligands. The oligonucleotides were filtrated and the supernatant concentrated by evaporation with a vacuum centrifuge and redissolved in mili-q water before purification by RP-HPLC.

Reversed phase HPLC was applied to purify the strands, the parameters details can be obtained in experimental part. The presence of failure strands indicates that the capping during the synthesis was not efficient enough. All short failure strands were separated by HPLC as can be observed by the chromatogram (Figure 46). There was a strong effect of peak broadening, especially for the strand $\mathbf{s} \mathbf{2}$ that is also in lower intensity, which made the separation of the peaks complicated and another HPLC run was required for this strand. The strand $\mathbf{s} 2$ remains still with a small shoulder near to the desired peak, indicating a slight 
impurity. The gradient adopted was previoulsy tested by a analytical column, but significant differences were obtained on the preparative system which also caused difficulties regarding the purification. After the chromatographic purification the samples were desalted and detritylated using Glen-Pak ${ }^{\mathrm{TM}}$ cartridges by Glen Research (according to instructions provided by the fabricant) and concentrated by vacuum centrifuge.
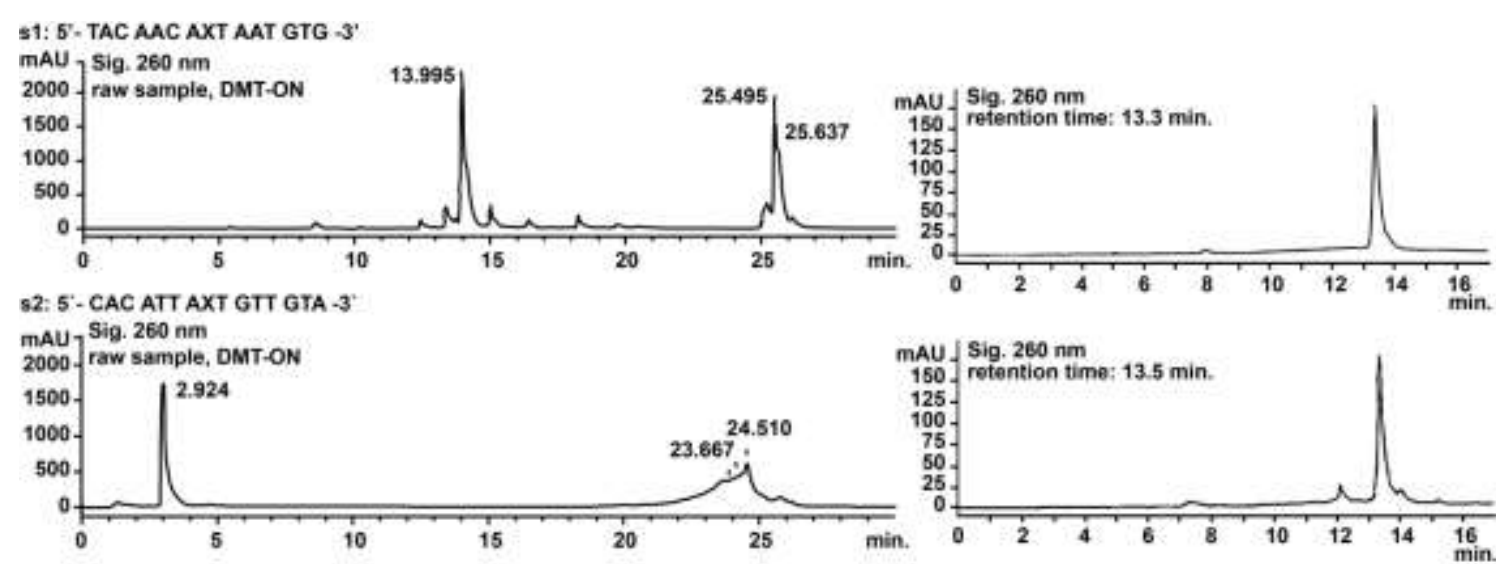

Figure 46. a) Analytical chromatogram for $\mathbf{s} 1$ and $\mathbf{s} \mathbf{2}$ raw sequences indicating fail strands and other small impurities, b) chromatogram for $\mathbf{s} \mathbf{1}$ and $\mathbf{s} \mathbf{2}$ after purification and DMT cleavage.

c) Determination of concentration and ESI-MS characterization:

The estimation of the concentration for the strands synthesized was performed by the base composition method ${ }^{[4,88]}$, which is simply the sum of the individual extinction coefficients of bases at $260 \mathrm{~nm}$ presented in a strand sequence, multiplied by a factor 0.9 (which takes into account base stacking in the strand causing a slight hypochromicity effect). The nearestneighbour method takes into account interactions between neighbour bases, because of it this method is not suitable for modified strands (for the same reason the use of nanodrop device is also not applied), since the value between the modified base with adjacent bases is unkown. Both methods show to be in agreement concerning the values obtained for unmodified strands.

The values for individual extinction coefficients were obtained by literature and presented as followed: $\mathrm{dA}=15.4, \mathrm{dC}=7.4, \mathrm{dG}=11.5$ and $\mathrm{dT}=8.7$ all values $* 10^{3} \mathrm{~L} \mathrm{~mol}^{-1} \mathrm{~cm}^{-1}$. ${ }^{[88]}$ For the modified base it is necessary to make an UV/Vis assay of the ligand (no DMT) under several concentrations $\left(0.001\right.$ to $0.08 \mathrm{mM}$ in $\mathrm{CHCl}_{3}$ ) where the slope obtained from the linear regression of the $A_{260}$ 's plotted is correspondent to the $\varepsilon_{260}$ for the individual artificial nucleoside. This quantification is in agreement with the Beer-Lambert law. ${ }^{[4]}$ For cases of 
concentrations values that present deviations from the linear regression the values should be avoided to work.

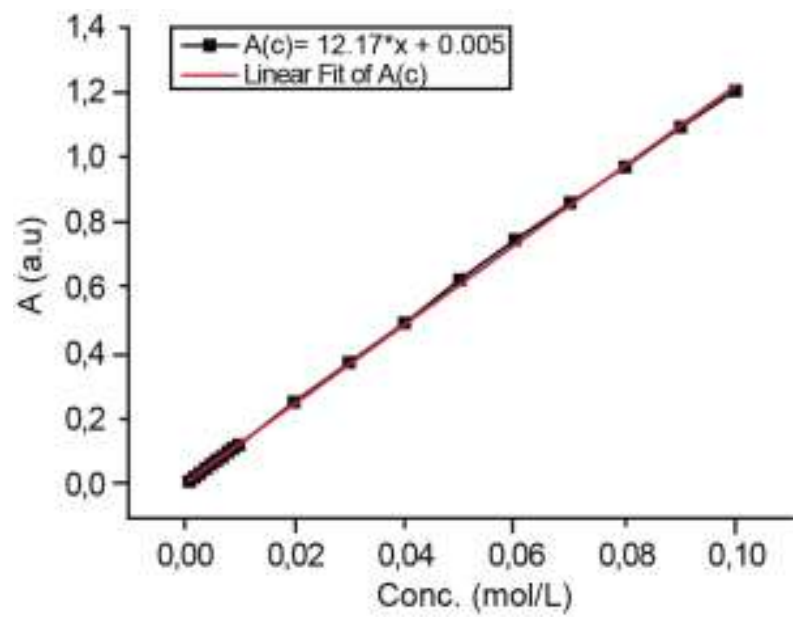

Figure 47. Linear regression of plotted values of absorption at $260 \mathrm{~nm}$ for ligand 1,2-bis(2ethynyl-5-hydroxyethoxypyridine)benzene, 4.

For the extinction coefficient, $\varepsilon_{260}=12.17 \times 10^{3}\left(\mathrm{~L} \mathrm{~mol}^{-1} \mathrm{~cm}^{-1}\right)$, using the base compostition method, the coefficient values for both strands are $154,453 \times 10^{3} \mathrm{~L} \mathrm{~mol}^{-1} \mathrm{~cm}^{-1}$ and $147,393 \times$ $10^{3} \mathrm{~L} \mathrm{~mol}^{-1} \mathrm{~cm}^{-1}$ for $\mathbf{s} \mathbf{1}$ and $\mathbf{s} 2$ respectively.

ESI-MS for the synthesized single strands $\mathbf{s} \mathbf{1}$ and $\mathbf{s} \mathbf{2}$ were performed after HPLC purification and appropriate desalting/detritylation. Mass spectra samples with a concentration of $20 \mu \mathrm{M}$ were prepared in aqueous solution (50:50 $\mathrm{H}_{2} \mathrm{O} / \mathrm{MeCN}$ ) and measured in negative ion mode at $150^{\circ} \mathrm{C}$. As can be observed in the Figure 48 the spectrum contains several peaks of negativelly charged patterns, related to the strand based on the phosphate-backbone where each deprotonated phosphate group contributes with a -1 charge. A simulation for the $\mathrm{m} / \mathrm{z}=-9$ pattern was calculated and is shown for both strands, where the observed slight shift of the peaks can be attributed to the machine calibration, not specific for DNA. It was also possible to observe in lower abundace peaks attributed to DNA adducts carrying $\mathrm{Na}^{+}$and $\mathrm{K}^{+}$ions. 


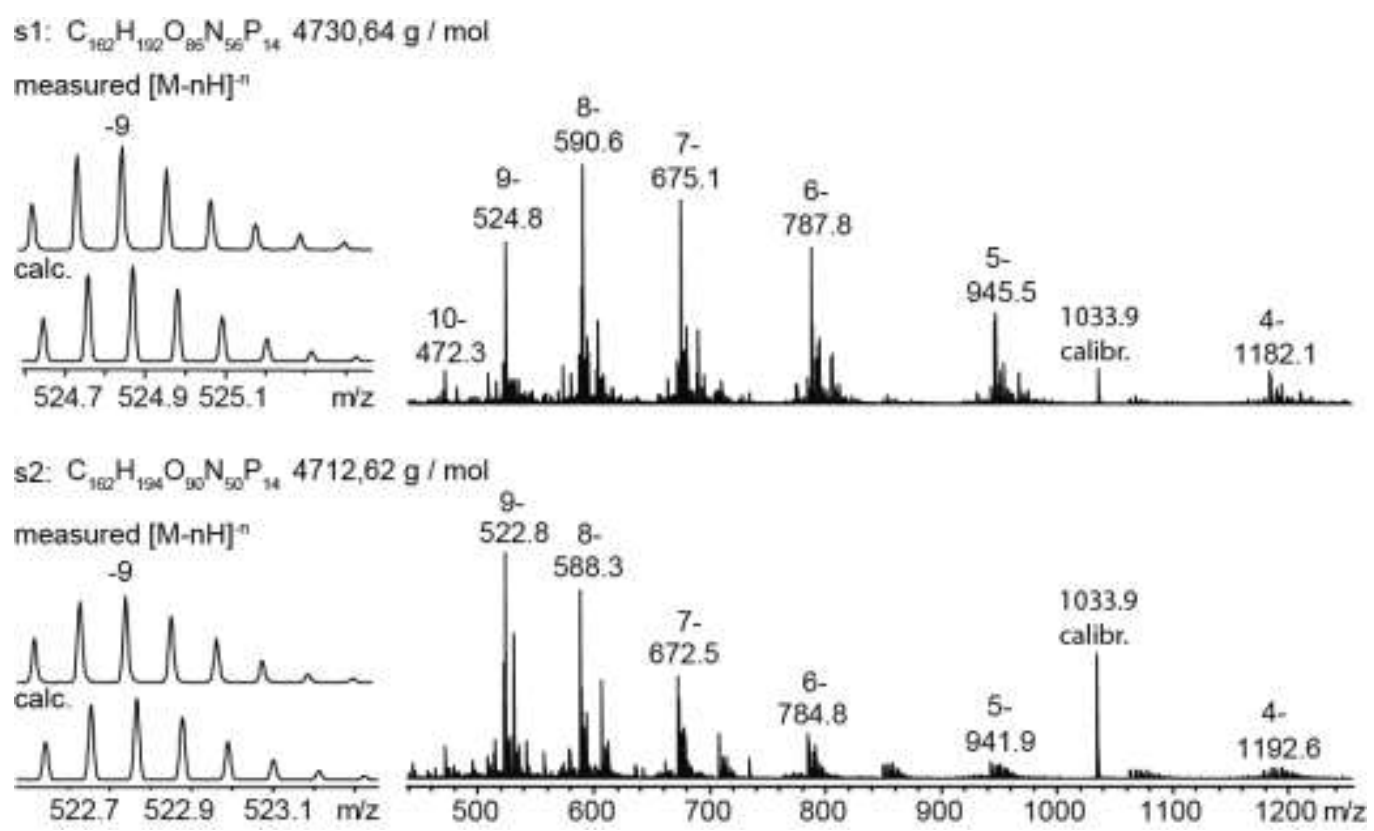

Figure 48. ESI-MS for $\mathbf{s} 1$ and $\mathbf{s 2}$ single strands and calculated and measured isotopic pattern for $m / z=-9$.

\subsubsection{Sample conditions and preparation}

DNA single strands $\mathbf{s} 1$ and $\mathbf{s} \mathbf{2}$ were combined in aqueous solution with different buffers (cacodylate buffer, 2-(cyclohexylamino)ethanesulfonic acid (CHES buffer), phosphate buffer) and different electrolytes $\left(\mathrm{NaCl}, \mathrm{NaClO}_{4}, \mathrm{MgCl}_{2}\right)$. The choice of the buffers was carefully done to avoid $\mathrm{pH}$ variations due to change in temperature, as previously observed for HEPES buffer (4-(2-hydroxyethyl)-1-piperazine-ethanesulfonic acid). ${ }^{[61]}$ Buffers that can act as ligands were also avoided to not compete with the artificial nucleotides on the binding to the species of metals added, as for example occurs for tris-buffer (tris(hydroxymethyl)aminomethane) and for phosphate buffer that is known to "kidnap" M(II) (depending on the binding constant of the artificial nucleotides) competing with the binding site of the artificial motifs for the $M(I I)$. In the presence of $\mathrm{MgCl}_{2}(10 \mathrm{mM})$ and other electrolytes with concentrations above $100 \mathrm{mM}$, the system indicates a higher stabilization than the final conditions chosen (electrolytes $100 \mathrm{mM}$ $\mathrm{NaCl}$ or $\mathrm{NaClO}_{4}$, although it was avoided because such conditions indicated a shift on the melting curve transition leading the formation of a secondary structure, which is discussed in more detail later (section 2.2.4). This observation indicates a dependence in ionic stregth competing for the formation of duplex structure. Previous assays with phosphate buffer did not show any change in the melting curve, however a slight change in absorbance after metal addition (several dications were tried) was observed, indicating that the buffer is probably sequestrating the metal species added and prohibiting the metal coordination with the target ligand. CHES buffer did not show any expressive change by thermal analysis. The duplex s1.s2 
displayed significant effective changes with $10 \mathrm{mM}$ cacodylate buffer (pH 7.4). Assays for the thermal analysis, UV/Vis, fluorescence and CD measurements were performed with the same sample and further metal additions ( $\mathrm{Cu}(\mathrm{II})$ and $\mathrm{Pd}(\mathrm{II}))$ having as standard conditions $3 \mu \mathrm{M}$ s1.s2, $10 \mathrm{mM}$ cacodylate buffer (pH 7.4) and $100 \mathrm{mM} \mathrm{NaCl}$ (for additions of copper) or $\mathrm{NaClO}_{4}$ (for additions of palladium). A blank solution (buffer and electrolyte) was measured for all spectroscopic methods applied. The sample was prepared combining both single strands and submitted to a slow incubation under slow cooling (details in experimental part). UV/Vis measurements were recorded before and after the thermal analysis. Metal additions were performed and the system was heated overnight at a temperature below the obtained melting point $\left(35-40^{\circ} \mathrm{C}\right)$ to avoid the melting of the duplex during metal coordination. The metal addition and its binding is promoted under a pre-organized system, where both artificial ligands are incorporated in the adjacent complementary strands.

\subsubsection{Thermal analysis and UV/Vis spectroscopy}

a) UV/Vis spectra for trans-ligand and complexes:

UV/Vis measurements for the trans-chelating ligand and its respective monomeric and dimeric complexes were discussed in the previous part 1, where it was presented the ${ }^{1} \mathrm{H}$ NMR spectra for the complexes formed with $\mathrm{Pd}(\mathrm{II})$ (Figure 33). ${ }^{[80]}$ The structure of the dimer complex of the trans-ligand with $\mathrm{Cu}(\mathrm{II})$ was elucidated by X-ray crystallography in the work carried out by Thummel et al.. ${ }^{[78]}$ The derivative ligand containing ethoxy-hydroxy groups attached at the 5 position in the 2-ethynyl-pyridine arms (as a linker for further steps previous to DNA solid phase synthesis) and its dimer and monomer complexes were also submitted to characterization by UV/Vis and ESI-MS despite the fact that it is not expected significant deviations from the derivative compounds presented in part 1. Because of solubility limitations, it was not possible to obtain UV/Vis data of the respective dimer and monomer complexes with $\mathrm{Cu}(\mathrm{II})$, been possible to collect UV/Vis data only for compounds made upon addition of $\mathrm{Pd}(\mathrm{II})$ in DMSO solution of the ligand (Figure 49). Addition of 0.5 eq. of $\mathrm{Pd}(\mathrm{II})$ into the ligand solution and heating for $4 \mathrm{~h}$ at $70^{\circ} \mathrm{C}$ led to the dimeric structure $\left[\mathrm{Pd}(\mathrm{Lig})_{2}\right]^{2+}$ (dashed red line in Figure 49) with a greater decrease in absorbance and different isosbestic point $(399 \mathrm{~nm})$ than the spectrum obtained for the monomeric complex prepared upon addition of 1 eq. of $\mathrm{Pd}(\mathrm{II})$ (393 nm, dashed blue line in Figure 49). The formation of the dimer and monomer complexes were attributed to the respective amounts of $\mathrm{Pd}(\mathrm{II})$ added into the ligand solution and based on the data obtained by the ${ }^{1} \mathrm{H}$ NMR (Figure 33 ) and mass values for its analogous system presented in part 1 where such equimolar amounts of metal into the ligand 
1,2-bis(2-ethynyl)benzene) were enough to obtain a full complexation in both cases of dimer and monomer complexes. Due to the bad volatilization of DMSO for ESI-MS measurements, the spectra for respective complexes with $\mathrm{M}(\mathrm{II})$ were not possible to be obtained.

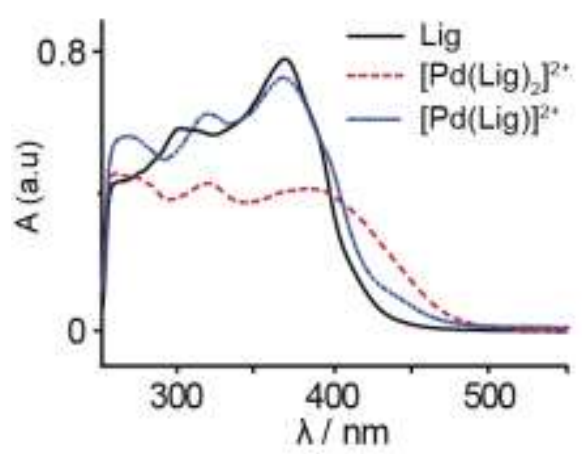

Figure 49. UV/Vis spectra for ligand 4, "Lig." and respectives monomeric and dimeric complexes with $\mathrm{Pd}(\mathrm{II}),(5.7 \mu \mathrm{M}$ in DMSO solution). Lig=1,2-bis(2-ethynyl-5-hydroxyethoxypyridine)benzene.

b) Thermal analysis and UV/Vis for modified DNA s1.s2:

The incorporation of artificial ligands into DNA and the further complexation with metal species through metal-base pairing formation normally provides extra stability to the system, which can be analysed by thermal analysis by observing the changes in the respective transition profile and melting point. The synthesized oligonucleotides were submitted to the analytical method under several conditions (buffers, electrolytes and $M(I I)$ species) to explore the potential of metal-base pairing been detailed on this section. Complementary to the melting curves, UV/Vis measurements were performed to obtain more data concerning the complexation of the ligands and $M(I I)$ added. In UV/Vis spectrum for $\mathbf{s 1 . s 2}$ an absorbance signal at $300-400 \mathrm{~nm}$ is attributed to the ligand and changes on this region after addition of $\mathrm{M}(\mathrm{II})$ are related to the complexation.

Control measurements were performed for single strands individually, by UV/Vis and melting curve analysis, with the purpose to discard possible secondary structures such as unspecific binding with $\mathrm{M}(\mathrm{II})$ (also measured for unmodified duplex, $\mathbf{s} \mathbf{1}^{*} . \mathbf{s 2} \mathbf{2}^{*}$. ${ }^{[89]}$ Melting curves for single strand s1 indicated an intramolecular equilibrium (Figure 50a), which means the melting point is independent of the concentration of the sample and it is characteristic for the hairpin structure (Figure 50b). ${ }^{[60]}$ 
a)

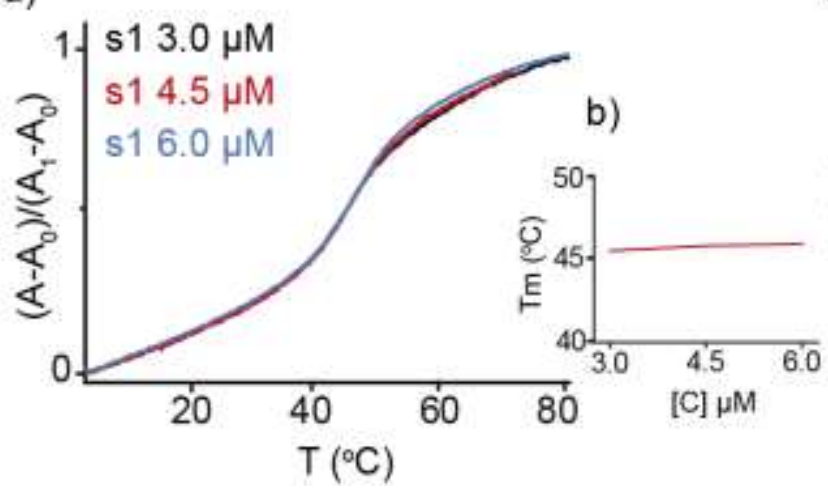

c)

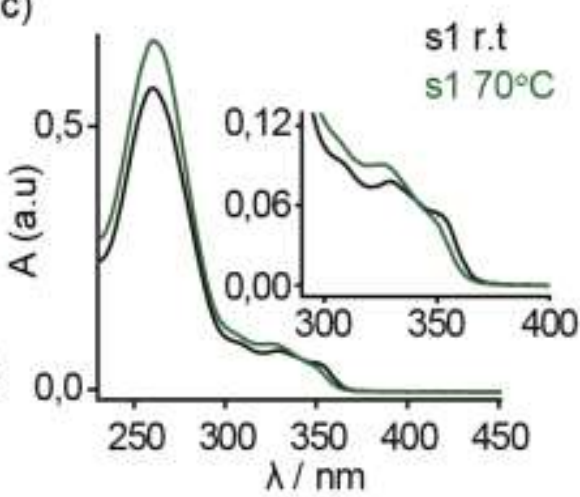

Figure 50. Melting curve analysis for the single strand $\mathbf{s} 1$ at $3.0,4.5$ and $6.0 \mu \mathrm{M}, \mathrm{b}$ ) plotting of Tm values vs concentration $(\mu \mathrm{M})$ indicating possible formation of a hairpin structure. $10 \mathrm{mM}$ cacodylate buffer, $100 \mathrm{mM} \mathrm{NaCl}$. c) UV/Vis for $3.0 \mu \mathrm{M}$ s1 at r.t. and $70^{\circ} \mathrm{C}$ indicating hyperchromism effect at $260 \mathrm{~nm}$.

The UV/Vis spectra measured at room temperature (hairpin) and also at $70^{\circ} \mathrm{C}$ (unfolded strand) indicate a hyperchromism effect at $A_{260}$ and a shifting of the bands attributed to the ligand region (300-400 nm). Melting curves of the single strand $\mathbf{s} 1$ were done upon adittion of $\mathrm{Cu}(\mathrm{II})(3.0 \mu \mathrm{M}$, cacodylate buffer $10 \mathrm{mM}, \mathrm{NaCl} 100 \mathrm{mM})$ and any change on the stability of the secondary structure was obtained due to metal complexation, the melting curve profile and melting point remain constant.

Thermal analysis for the duplex s1.s2 (3 $\mu \mathrm{M})$ in cacodylate buffer $(10 \mathrm{mM})$ with $\mathrm{NaCl}(100 \mathrm{mM})$ indicated a change in the duplex stability equivalent to $\Delta \mathrm{Tm}=-1^{\circ} \mathrm{C}$ in comparison to the unmodified system $\mathbf{s 1 *} . \mathbf{s} \mathbf{2}^{*}$. Despite the fact that the curve profile indicates a broader transition than its unmodified analogue duplex $\left(\mathbf{s} \mathbf{1}^{*} . \mathbf{s 2} \mathbf{2}^{*}\right)$, it is a very slight destabilization for a system with a "break" on the Watson-Crick base pairing right in the middle of the duplex. Artificial ligands incorporated into the DNA have to be designed such that the possible distortions on the base stacking of the DNA system, are as minimal as possible.
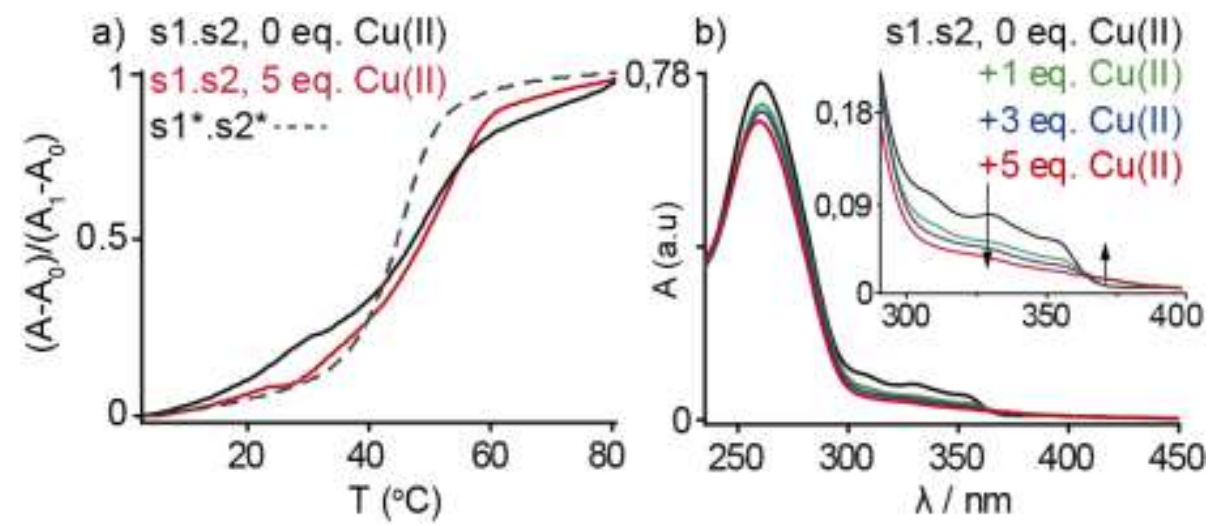

Figure 51. a) Thermal analysis for $3.0 \mu \mathrm{M}$ unmodified duplex s1*.s2*, $10 \mathrm{mM}$ cacodylate buffer (pH 7.4), $100 \mathrm{mM} \mathrm{NaCl}$ and under same conditions melting curve for duplex s1.s2, + 
n eq. Cu(II). b) UV/Vis for $3.0 \mu \mathrm{M}$ s1.s2 + n eq. Cu(II), $10 \mathrm{mM}$ cacodylate buffer (pH 7.4), $100 \mathrm{mM} \mathrm{NaCl}$.

The trans-chelating ligand incorporated indicates a suitable adjustment to the system (more details about it in CD spectroscopy) based on the value obtained for the melting point. After addition of 5 eq. of $\mathrm{Cu}\left(\mathrm{II}\right.$ ), the system s1.s2 shows a higher stability with $\Delta \mathrm{Tm}=+6.5^{\circ} \mathrm{C}$ (as expected after formation of metal-base pairing). Further additions of $\mathrm{CU}(\mathrm{II})$ does not cause relevant changes, which indicates a possible full coordinated system. The conclusion was complemented by UV/Vis measurements (Figure 51b) which show that after additons of $\mathrm{Cu}(\mathrm{II})$ the absorbance on the region assigned for the ligand presents a drop in absorbance with an isosbestic point around $\lambda=362 \mathrm{~nm}$ that starts to occur with addition of 1 eq. of $\mathrm{Cu}(\mathrm{II})$. The drop in absorbance is lower for further additions of $\mathrm{CU}(\mathrm{II})$ and is not more efficient for higher values than 5 eq. of $\mathrm{Cu}(\mathrm{II})$. A slight increasing in absorbance after the isosbestic point ( $363 \mathrm{~nm}$ ) was also noticed until a range around $390 \mathrm{~nm}$.

The excess of copper(II) provides a complete coordinated system (the dimer complex should be complete coordinated upon addition of 0.5 eq. of $\mathrm{Cu}(\mathrm{II})$, amont that did not demonstrated any change by UV/Vis spectroscopy or in the melting point profile in comparison to the system at 0 eq. of $\mathrm{Cu}(\mathrm{II}))$ can be due to a low binding constant for the complex, buffer conditions or an unspecific binding in the DNA helix competing with the binding sites of the ligand. Nevertheless, it is not expected that the excess of copper(II) can cause the formation of monomeric structures. This possibility is discarded because the system is pre-organized in a way that facilitates the dimeric form, with both ligands placed facing each other. It was observed data by thermal analysis and CD spectroscopy that support the integrity of the duplex structure, been sustained and presenting an extra stability, according to the melting analysis, due to the metal-base pairing formation. Evidence of a monomeric complex or any another significant change could be observed by UV/Vis spectroscopy under the appearance of another isosbestic point or change in the intensity of the absorption, which did not happened.

The melting curve after 5 eq. of $\mathrm{Cu}(\mathrm{II})$ also shows a slight improvement on the sharpness for the transition. The levels in the curve considering a fully melted and fully annealed structure presented a higher hyperchromism and higher hypochrosmism, respectively. For duplex s1.s2 the presence of $\mathrm{MgCl}_{2}$ ( $3 \mu \mathrm{M}$ DNA, cacodylate buffer $10 \mathrm{mM}, \mathrm{NaCl} 100 \mathrm{mM}$ and $10 \mathrm{mM} \mathrm{MgCl}$ ) indicates a much higher stabilization for the system, which is consistent with the fact $\mathrm{Mg}(\mathrm{II})$ cations can provide better stabilization for the double helix than monocations; they are divalent ions and can screen better the phosphate backbone (softer ion which contributes too 
for a greater stability). Although, after addition of $\mathrm{Cu}(\mathrm{II})$ it is possible to observe by UV/Vis a lower drop in the absorbance intensity for the ligand region with $\mathrm{Mg}(\mathrm{II})$ in comparison to assay perfomed in the absence of $\mathrm{Mg}(\mathrm{II})$. One hypothesis for a less efficient binding is the fact that magnesium(II) may be competing with copper(II) to coordinate with the ligands. The melting profile also indicates a lower extra stabilization after addition of 5 eq. of $\mathrm{Cu}(\mathrm{II})$, been $+2.2^{\circ} \mathrm{C}$.
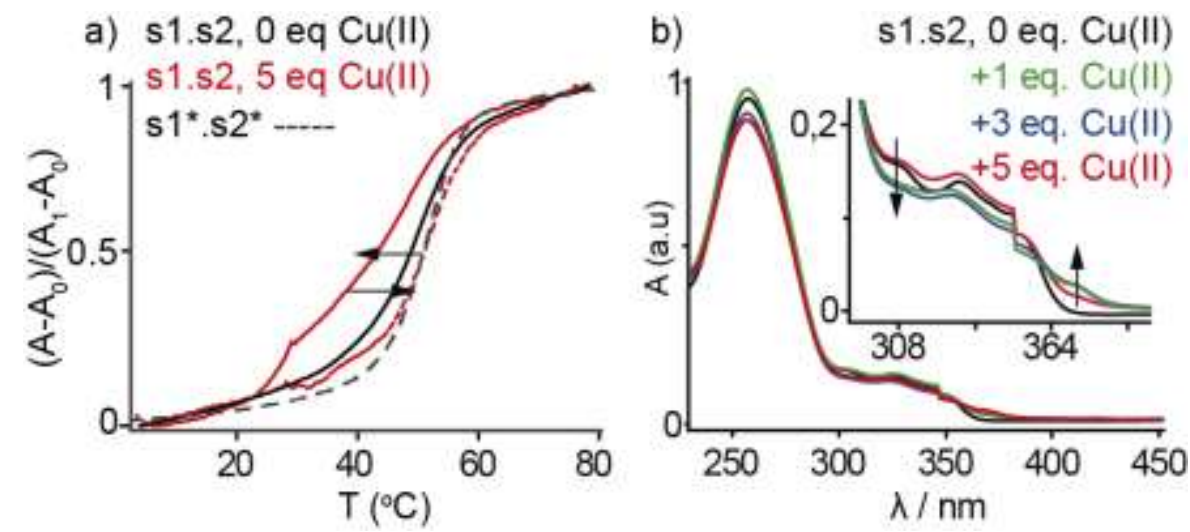

Figure 52. a) Thermal analysis for a $3.0 \mu \mathrm{M}$ solution of the unmodified duplex s1*.s2*, $10 \mathrm{mM}$ cacodylate buffer ( $\mathrm{pH}$ 7.4), $100 \mathrm{mM} \mathrm{NaCl}, 10 \mathrm{mM} \mathrm{MgCl}_{2}$, and under same conditions melting curve for duplex s1.s2, + n eq. Cu(II). b) UV/Vis for $3.0 \mu \mathrm{M}$ s1.s2 + n eq. Cu(II), $10 \mathrm{mM}$ cacodylate buffer (pH 7.4), $100 \mathrm{mM} \mathrm{NaCl}, 10 \mathrm{mM} \mathrm{MgCl}$.

Initialy, right after the copper(II) addition, it is possible to see on the first melting curve (red solid line, Figure 52a) a strong destabilization, but already on the reannealing cycle the system becomes more stable (red dashed line Figure 52) and this profile stays for further thermal cycles, indicating a reproducible process and with a similar kinetic path for melting and annealing, due to the lack of hysteresis. Possibly at the first melting cycle, the $\mathrm{Cu}(\mathrm{II})$ was not fully coordinated to the ligands and the competition with $\mathrm{Mg}(\mathrm{II})$ caused a destabilization, which after time and heating the binding was finished and the formation of MBP provided a more stable duplex.

A similar assay was prepared for the addition of $\mathrm{Pd}(\mathrm{II})$. The electrolyte was changed to $\mathrm{NaClO}_{4}$ to work with palladium(II), which is a softer cation than copper(II). The change of $\mathrm{NaCl}$ to $\mathrm{NaClO}_{4}$ did not cause notable differences in the melting profile or the stability of the duplex, the melting curve temperature was $45.5^{\circ} \mathrm{C}\left(\Delta \mathrm{Tm}=+0.5^{\circ} \mathrm{C}\right)$. After addition of 5 eq. of $\mathrm{Pd}(\mathrm{II})$ the curve showed less stabilization, with a $\Delta \mathrm{Tm}=-3.9^{\circ} \mathrm{C}$. The UV/Vis spectra indicated a drop in the absorption for the ligand region after each addition of $\mathrm{Pd}(\mathrm{II})$, but less expressive than the drop of the absorption (Figure 53b) observed for the assay with $\mathrm{Cu}(\mathrm{II})$ (almost flattened ligand region with 5 eq. of $\mathrm{Cu}(\mathrm{II}))$, with the isosbestic point remaining at $362 \mathrm{~nm}$. 
a) $s 1 . s 2,0$ eq Pd(II)

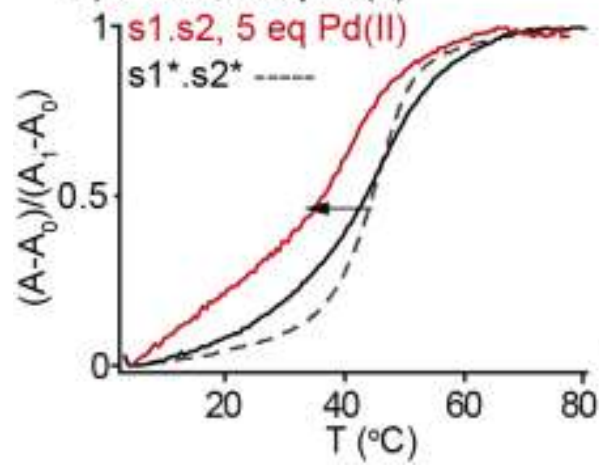

b)

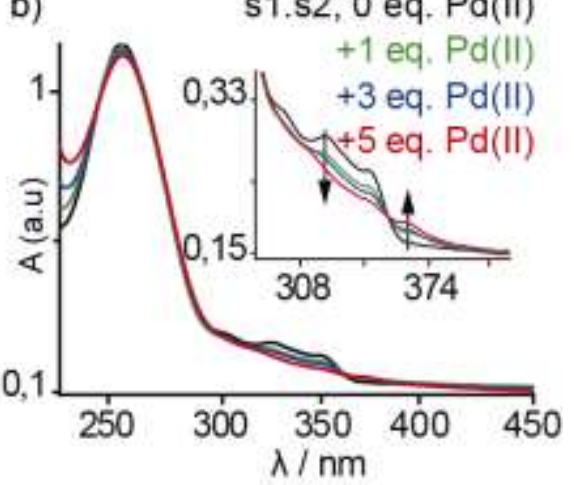

Figure 53. a) Thermal analysis for $3.0 \mu \mathrm{M}$ unmodified duplex s1*.s2*, $10 \mathrm{mM}$ cacodylate buffer ( $\mathrm{pH} 7.4), 100 \mathrm{mM} \mathrm{NaClO}_{4}$ and under the same conditions for melting curve of the duplex s1.s2, + n eq. $\mathrm{Pd}(\mathrm{II})$. b) UV/Vis for $3.0 \mu \mathrm{M}$ s1.s2 + n eq. $\mathrm{Pd}(\mathrm{II}), 10 \mathrm{mM}$ cacodylate buffer (pH 7.4), $100 \mathrm{mM} \mathrm{NaClO}_{4}$.

To provide more evidence that such observed effects come from Pd(II) and not because of the change of the electrolyte, the assay was repeated with $\mathrm{Cu}(\mathrm{II})$ and $\mathrm{NaClO}_{4}$. The UV/Vis is similar to the analogous assay of $\mathrm{Cu}(\mathrm{II})$ with $\mathrm{NaCl}$ (Figure 51b). The melting curve is also more stable than in the case of addition of $\mathrm{Pd}(\mathrm{II})$, with a $\Delta \mathrm{Tm}=+4.5^{\circ} \mathrm{C}$ (Figure 54a). Although this value indicated lower stabilization than the similar assay of $\mathrm{Cu}(\mathrm{II})$ performed with $\mathrm{NaCl}$ as electrolyte. This assay gave more information about the system indicating a possible preference for harder ions as electrolyte and harder cations as Lewis acids to coordinate with the ligand.

When the metal-base pairing provides extra stability for the system, as in the case for s1.s2.Cu(II), it indicates that the complex formation does not cause distortions of the double helix. In contrast, cases of destabilization are always related to the effects of distortion, ${ }^{[4,86]}$ as happened to the assay performed with $\mathrm{Pd}(\mathrm{II})$. The complex formed through s1.s2.Pd(II) might cause distortions related to the size and geometry of the square-planar structure giving extenended distortions also to the neighbouring base pairs and stacking, compromising the whole duplex. 
a) s1.s2, 0 eq. $\mathrm{Cu}(\mathrm{II})$

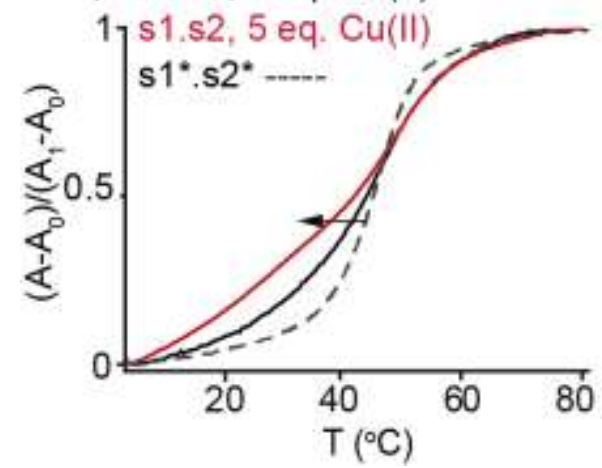

b)

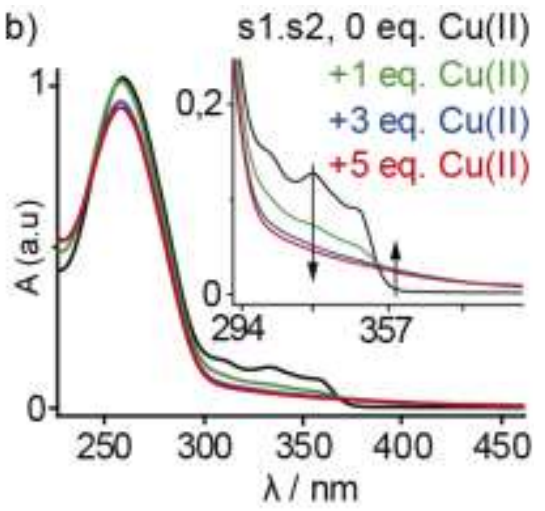

Figure 54. a) Thermal analysis of a $3.0 \mu \mathrm{M}$ solution of the unmodified duplex s1*.s2*, $10 \mathrm{mM}$ cacodylate buffer ( $\mathrm{pH}$ 7.4), $100 \mathrm{mM} \mathrm{NaClO}$ and under same conditions melting curve for duplex s1.s2, + n eq. Cu(II). b) UV/Vis for $3.0 \mu \mathrm{M}$ s1.s2 + $\mathrm{n}$ eq. $\mathrm{Cu}(\mathrm{II}), 10 \mathrm{mM}$ cacodylate buffer (pH 7.4), $100 \mathrm{mM} \mathrm{NaClO}_{4}$.

Table 2. Melting point values and conditions for several systems.

\begin{tabular}{|c|c|c|c|c|c|}
\hline duplex & electrolyte & Metal & $n$ eq.of $M(I I)$ & $\operatorname{Tm}\left({ }^{\circ} \mathrm{C}\right)$ & ${ }^{*} \Delta \operatorname{Tm}\left({ }^{\circ} \mathrm{C}\right)$ \\
\hline \multirow[t]{7}{*}{ s1.s2 } & $\mathrm{NaCl}$ & - & 0 & 45.0 & $-1.0^{*}$ \\
\hline & & $\mathrm{Cu}(\mathrm{II})$ & 5 & 51.5 & +6.5 \\
\hline & $\mathrm{NaCl}+\mathrm{MgCl}_{2}$ & & 0 & 50.1 & $-1.9 *$ \\
\hline & & $\mathrm{Cu}(\mathrm{II})$ & 5 & 52.3 & +2.2 \\
\hline & $\mathrm{NaClO}_{4}$ & & 0 & 45.5 & $-0.2^{*}$ \\
\hline & & $\mathrm{Cu}(\mathrm{II})$ & 5 & 50.0 & +4.5 \\
\hline & & $\operatorname{Pd}(I I)$ & 5 & 41.6 & -3.9 \\
\hline \multirow[t]{3}{*}{ s1*.s2* } & $\mathrm{NaCl}$ & - & 0 & 46.0 & - \\
\hline & $\mathrm{NaCl}+\mathrm{MgCl}_{2}$ & - & 0 & 52.0 & - \\
\hline & $\mathrm{NaClO}_{4}$ & - & 0 & 45.7 & - \\
\hline
\end{tabular}

*Values for DNA thermal stability for modified oligonucleotides in comparison to unmodified analogues.

\subsubsection{Fluorescence spectroscopy}

Excitation at $330 \mathrm{~nm}$ for the same samples submitted for thermal analysis and UV/Vis measurements demonstrated complementary data concerning the metal complexation, whereas the emission spectra (Figure 55, 340-600 nm) indicated progressive quenching of fluorescence upon addition of rising amounts of $M(I I)$. The percentage of quenching was calculated based on literature ${ }^{[67]}$ and are summarized below in table 3 . Interestingly, the duplex sample with $\mathrm{NaClO}_{4}$ as the electrolyte presents a higher quenching for the addition of $\mathrm{Cu}(\mathrm{II})$ than the duplex with $\mathrm{NaCl}$. It is worth to remember, although, that the stabilization of 
the duplex for the system with $\mathrm{NaCl}$ was characteristic of a slighty greater value while the UV/Vis for both systems are quite similar.
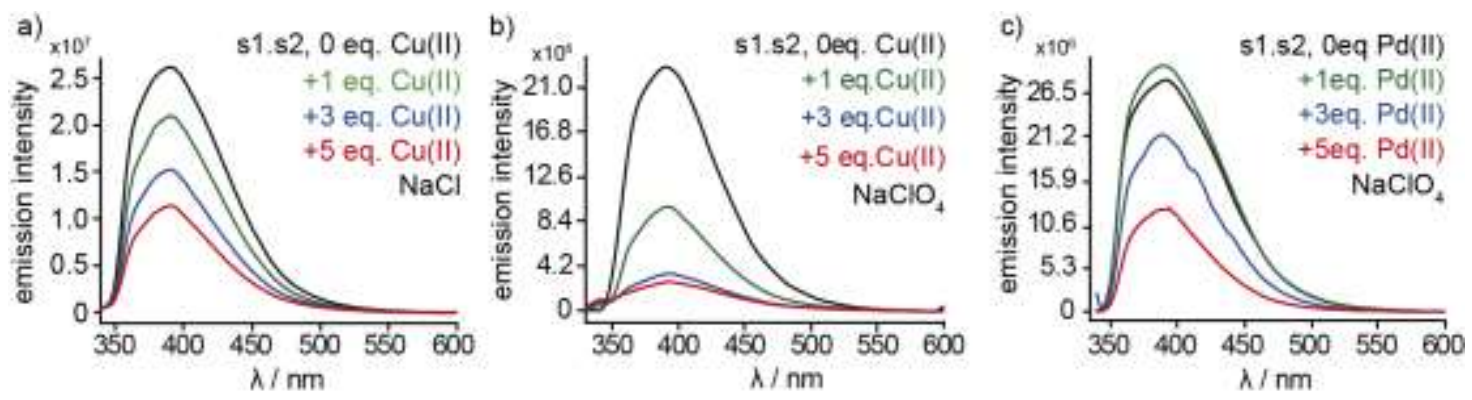

Figure 55. Emission spectra for $3.0 \mu \mathrm{M}$ s1.s2 + n eq. M(II), $10 \mathrm{mM}$ cacodylate buffer (pH 7.4), a) $100 \mathrm{mM} \mathrm{NaCl}, \mathrm{M}(\mathrm{II})=\mathrm{Cu}(\mathrm{II})$, b) $100 \mathrm{mM} \quad \mathrm{NaClO}_{4}, \mathrm{M}(\mathrm{II})=\mathrm{Cu}(\mathrm{II})$, c) $100 \mathrm{mM} \mathrm{NaClO}$, $M(I I)=P d(I I)$. Excitation $330 \mathrm{~nm}$, emission $340 \mathrm{~nm}$.

Table 3. Percentage for quenching upon metal addition

\begin{tabular}{cccc}
\hline $\begin{array}{c}\text { \% Emission } \\
\text { Quenched }\end{array}$ & $\mathrm{NaCl}, \mathrm{Cu}(\mathrm{II})$ & $\mathrm{NaClO}_{4}, \mathrm{Cu}(\mathrm{II})$ & $\mathrm{NaClO}_{4}, \mathrm{Pd}(\mathrm{II})$ \\
\hline 1 eq. & $20.1 \%$ & $57.2 \%$ & $+6.1 \%$ \\
3 eq. & $41.6 \%$ & $84.3 \%$ & $23.5 \%$ \\
5 eq. & $56.3 \%$ & $87.8 \%$ & $55.9 \%$ \\
\hline
\end{tabular}

\subsubsection{Circular dichroism spectroscopy}

CD spectroscopy for the equimolar ratio of $\mathbf{s} \mathbf{1}$ and $\mathbf{s} \mathbf{2}$ under slow cooling to allow the system to hybridize, indicates expected Cotton effects in the absorption region related to the B-DNA form (negative band around $245 \mathrm{~nm}$ and positive band at $275-280 \mathrm{~nm}$ ) for all the samples tried with cacodylate buffer $(10 \mathrm{mM})$ and $\mathrm{NaCl}(100 \mathrm{mM})$ or $\mathrm{NaClO}_{4}(100 \mathrm{mM}) .{ }^{[69,71]}$ The intensity of the $C D$ bands are higher than the respective bands for the single strands, which is expected because of the $\pi$ stacking between adjacents bases and hydrogen interactions established in the helical duplex structure. The CD spectrum for $\mathbf{s 1 . s 2}$ shows an additional band coherent to the region of the ligand absorption, in the range of $320-360 \mathrm{~nm}$, a region further than the characteristic band corresponding to the double helix profile. The trans-ligand itself is achiral, but it is known that the helical DNA environment transfer chirality onto incorporated ligands. ${ }^{[86,90]}$ The Figure 56 show the spectra for the assay with $100 \mathrm{mM} \mathrm{NaCl}$ (similar spectra for $\mathrm{NaClO}_{4}$ were obtained and can be observed in appendix (Figure 98). The CD spectrum for $\mathbf{s 1}$ also indicates, at room temperature measurement, the additional band related to the ligand 
region (Figure 56a). This band is not observed for the complementary strand $\mathbf{s} 2$ and this is reasonable due to the fact that it is not a double helix structure. So why such band was observed in the spectrum for the single strand s1? Melting curve studies carried out for the single strand indicated for $\mathbf{s 1}$ an intramolecular equilibrium, a secondary structure, possibly a hairpin or a $\pi$-stacked intramolecular aggregate. Furthermore, based on CD spectrum it is suspected that the ligand is also part of this system because of the extra band observed. The $\mathrm{CD}$ spectra for both single strands were repeated at $70^{\circ} \mathrm{C}$ and for strand $\mathbf{s} 1$ it is expected that the secondary structure is unwinded and the chirality transfer effect will no longer be observed, as indeed happens; the band at 320-360 nm vanishes. A decrease in intensity for the negative band was also noticed and it is assumed as a lack of nucleobase interactions related to the secondary structure now that the sequence is unfolded.

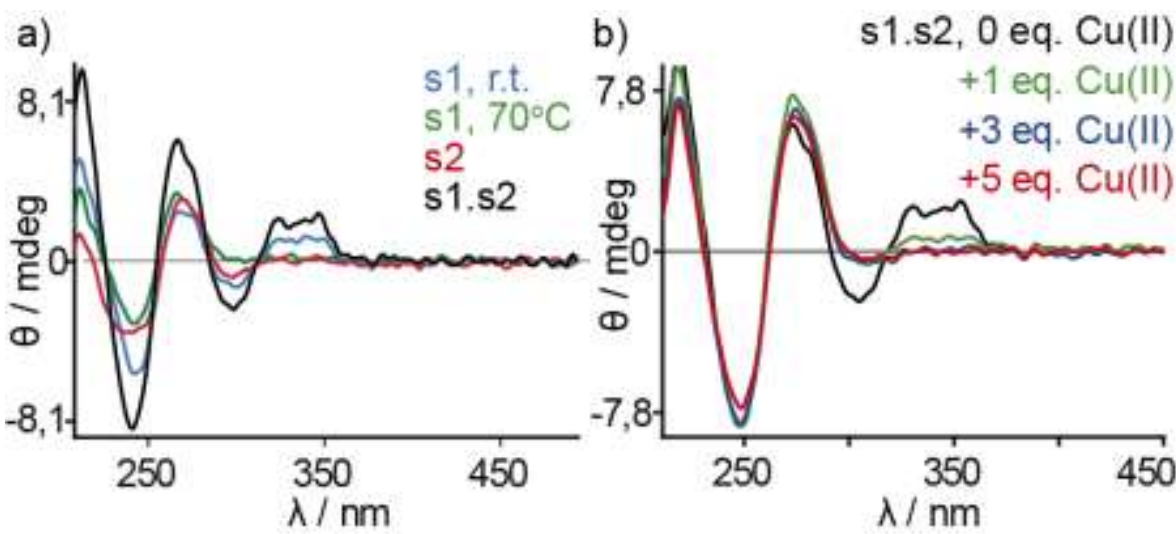

Figure 56. $\mathrm{CD}$ spectra for a) $3.0 \mu \mathrm{M} \mathbf{s} 1$ (room temperature and at $70^{\circ} \mathrm{C}$ ), $\mathbf{s 2}$, and duplex $\mathbf{s 1 . s 2}$, $10 \mathrm{mM}$ cacodylate buffer (pH 7.4), $100 \mathrm{mM} \mathrm{NaCl}$ b) $3.0 \mu \mathrm{M}$ s1.s2 + n eq. Cu(II), $10 \mathrm{mM}$ cacodylate buffer (pH 7.4), $100 \mathrm{mM} \mathrm{NaCl}, 100 \mathrm{mM}$. Excitation wavelength $330 \mathrm{~nm}$, emission wavelength $340 \mathrm{~nm}$.

The effects upon metal addition of the s1.s2 duplex can be observed in the CD spectra (Figure 56b) the region assigned for the ligand decreases in intensity for every addition of $\mathrm{Cu}(\mathrm{II})$ (this profile is also obtained for $\mathrm{Pd}(\mathrm{II}))$. After the addition of 5 equivalents of $\mathrm{Cu}(\mathrm{II})$ the signal was completely supressed. This is probably caused not by the decrease of helicity due to metal coordination (square-planar complex), but it is rather related to the complex formed which possesses a propeller shape. As discussed before for the UV/Vis measurements, the complexation providing a square-planar complex as metal-base pairing may cause distortion on the system and not contribute to base stacking with neighbouring bases. This however is not observed for the addition of $\mathrm{Cu}(\mathrm{II})$, which actually provides extra stabilization of the system. This is possible to occur if we take into account the propeller shape of the complex (molecular model in Figure 45) and the vanishing of the ligand band in CD spectra which might be related to a simpler fact that is the drop of the absorption intensity as already observed in 
the UV/Vis spectra in section 2.2.4. The system and complex shape allow existence of diastereomers where the stereochemistry might be controlled by the DNA chirality. It is not possible therefore to deconvolute the signal and assign which of the isomers has been favorably formed. An equal formation of the isomers would cancel the signal. Deconvolution of the spectrum would require a deeper analysis including modeling studies.

\subsubsection{Electrospray ionization mass spectrometry for DNA duplex and metal-base pairing}

ESI mass spectrometry was applied to analyse cross-links of single and double strands with $M(I I)$. Several measurements carried out with a large possibility of conditions and metal species ( $\mathrm{Cu}(\mathrm{II}), \mathrm{Pd}(\mathrm{II}), \mathrm{Ni}(\mathrm{II}), \mathrm{Zn}(\mathrm{II}))$ were tested and many inconclusive data were obtained before cross-links with $\mathrm{Cu}(\mathrm{II})$ were detected. The analysis of the DNA duplex by ESI-MS was already obtained, and there are several examples in the literature, including adducts based on non-covalent interactions, where small aromatic molecules and complexes interact with major or minor grooves of the DNA helix. ${ }^{[73,74]}$ Nevertheless, the conditions to obtain the duplex presented to be quite challenging; the volatilization of the molecules to produce a mist of droplets for measurements compromises the duplex integrity, due to the fact that the temperature is high enough to promote denaturation. Assays based on buffered samples with $100 \mathrm{mM}$ ammonium acetate $(\mathrm{pH} 8.3)$ demonstrated to be sucessful, but it is also important to take into account the spectrometer and certain conditions associated with the measurement, such as the collision cell energy $(8 \mathrm{eV})$ and temperature $\left(150-100^{\circ} \mathrm{C}\right)$ which were able to provide milder ionization conditions and thus some duplex structure could be detected.

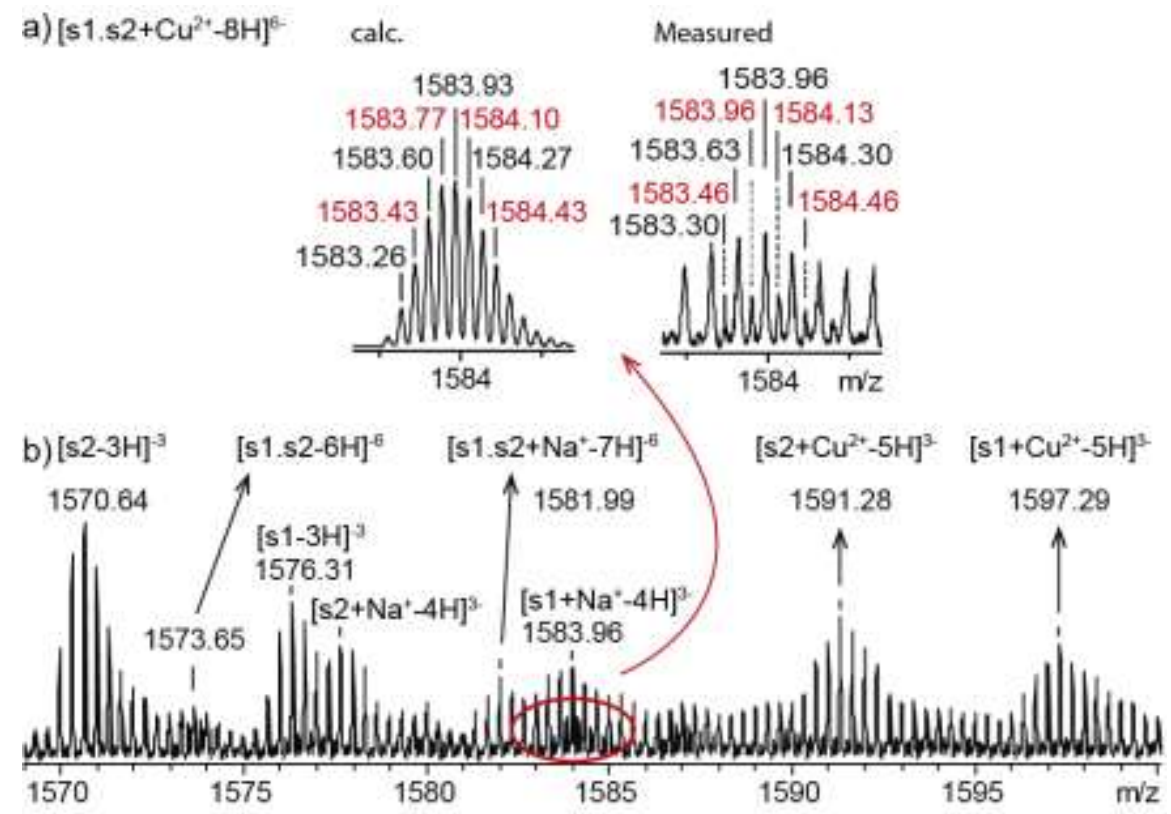

Figure 57. ESI-MS for $20 \mu \mathrm{M}$ s1.s2 and metal adducts with $\mathrm{Cu}(\mathrm{II})$. 
The duplex can be observed in a lower abundance than the correspondening complementary disrupted single strands (Figure 57b). It can be confirmed that the distance of the peaks in the isotopic pattern for double strand has the double of the distance (such as charge value) than the correspondent patterns for single strands, as for example could be noticed for [s1.s2-6H $]^{6-}$ and single strans $[\mathbf{s} 1-3 \mathrm{H}]^{3-}$ and $[\mathbf{s 2}-3 \mathrm{H}]^{3-}$ in Figure 57 above. An advantage of ESI-MS over MALDI is the fact that it is obtained a set of charges (MALDI would present a single peak) and also allows us to have a deeper analysis about the isotopic pattern and adducts, carring sodium and potassium adducts of the species (could be observed $\left[\mathbf{s 1 . s 2}+\mathrm{Na}^{+}-7 \mathrm{H}\right]^{6-}$ ), where all the peaks sustain the isotopic pattern and present a set of possible charges based on the negative charged phosphate-backbone. The duplex was properly incubated under ammonium acetate buffer and slowly cooled before addition of $\mathrm{Cu}(\mathrm{II})$ and submitted to a milder heating condition (below the melting point). This was carried out to provide the metal complexation without denaturation of the duplex or formation of secondary structures (hairpin for $\mathbf{s} \mathbf{1}$, for example and thus promote dimeric over monomeric complexes with the single strands.

Based on other spectrocopic studies, it is not expected that with only 1 eq. of $\mathrm{Cu}$ (II) full complexation occurs, but an excess greater than this amount did not help to produce good mass spectra. The excess of salts and ions increased the noise of the spectrum and therefore proper assignment of peaks could not be performed. Due to this, it is expected that for 1 eq. of $\mathrm{Cu}(\mathrm{II})$ remains unmetallated adducts (single and double strands) which indeed were observed. Monomeric $\mathrm{CU}(\mathrm{II})$ complexes respectives for both single strands were observed, $[\mathbf{s} \mathbf{1}+\mathrm{Cu}(\mathrm{II})$ $5 \mathrm{H}]^{3-}$ and $[\mathbf{s} 2+\mathrm{Cu}(\mathrm{II})-5 \mathrm{H}]^{3-}$ Figure $57 \mathrm{~b}$, as possibly the products of duplex denaturation during the ionization process. A single metal-base pairing on the system is not enough to hold the duplex together with respect to the temperature ionization conditions. Despite the lower relative abundance in comparison to the metallated single strand adducts, a remanescent minor abundance peak for the metal-base pairing was still detectable. The peak [s1.s2+Cu(II)$8 \mathrm{H}]^{-6}$ was detected and it is partially overlapped with $\left[\mathbf{s} 1+\mathrm{Na}^{+}-4 \mathrm{H}\right]^{3-}$. Diferentiation between them is possible because of the isotopic pattern; for the single strand the charge is -6 and for double strand is -3 , having them half the distance between the isotopic peaks than it is for single strand. The MBP presents peaks with lower abundance and it is intercalated by overlapping peaks from $\left[\mathbf{s} 1+\mathrm{Na}^{+}-4 \mathrm{H}\right]^{3-}$, which has higher intensity (Figure 57a).

\subsubsection{Polyacrylamide gel electrophoresis, PAGE}

Native polyacrylamide gel indicates more evidence of the secondary structure for single strand s1 where is possible to observe two spots (Figure 58). A sucessful hybridization under 
equimolar amounts of $\mathbf{s} \mathbf{1}$ and $\mathbf{s} \mathbf{2}$ was obtained. The modified strands exhibit a fluorescence upon UV irradiation a faint purple color but unfortunately not enough to be observed on the gel by UV shadow. The visualization was successful after a stain with SYBR green gel stain.

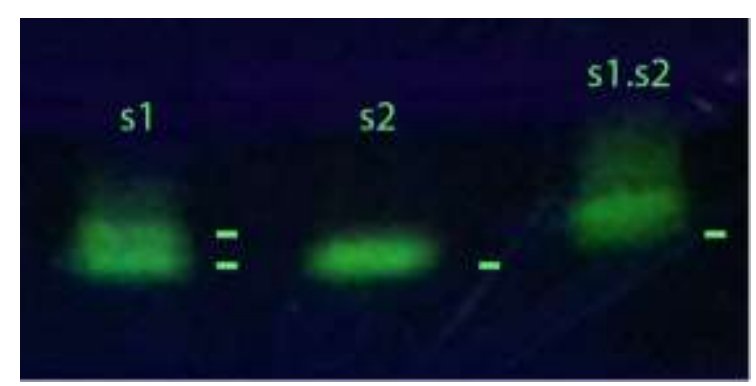

Figure 58 . Native $8 \%$ polyacrylamide gel with TBM buffer $(\mathrm{pH} 8,3)$ for single strands $\mathbf{s} \mathbf{1}, \mathbf{s} 2$ and duplex s1.s2.

Further attempts to obtain a gel with the metallated system (indicating formation of metalbase pairing) were performed. The lanes for $\mathbf{s} \mathbf{1}+\mathrm{Cu}(\mathrm{II})$ and $\mathbf{s} \mathbf{2}+\mathrm{Cu}(\mathrm{II})$ (lanes 2 and 4 respectivelly in Figure 59) did not show any change to the corresponding single strands without addition of $\mathrm{Cu}(\mathrm{II})$, probably due to the fact the mobility and values for molecular weigth stay quite similar.

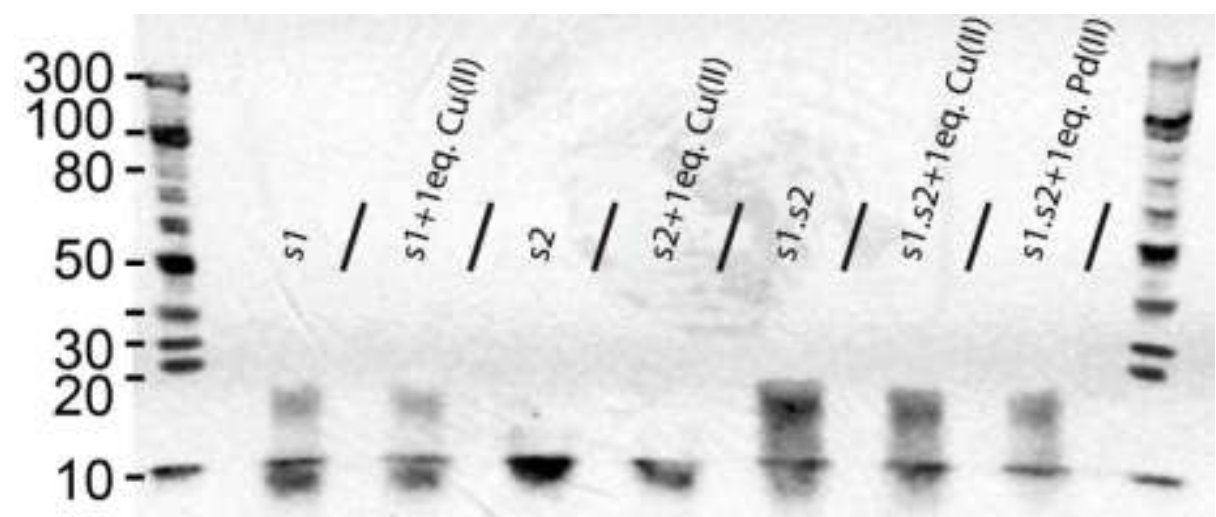

Figure 59. Native 8\% polyacrylamide gel with TBM buffer ( $\mathrm{pH} 8.3)$ for the single strands $\mathbf{s 1}, \mathbf{s 2}$ and duplex s1.s2 and for the respectives attempts of M(II) complexation.

For lanes of s1.s2+Cu(II) and $+\mathrm{Pd}(\mathrm{II})$ (lane 6 and 7 respectively) it is not clear if the duplex hybridization was not fully acquired due to the presence of the lower bands (around $10 \mathrm{~b}$ of the marker) which could also be metallated the single strand s1 or s2. Again, the spots attributed to s1.s2 (upper spot) did not show significant change relative to the corresponding unmetallated system (lane 5) which gives to the undenaturing gel an inconclusive result. 


\subsection{Aims of the project (Part 3) - Artificial trans-chelating nucleoside poiting outside of the DNA helix and DNA inter cross-link through metal complex formation}

The design of DNA nanoconstructs exploiting the potential and new possibilities of systems with new applications through the metal complexation and the incorporation of artificial ligands and nucleotides into the DNA has gained several progresses, such as DNA nanotubes, DNA branches and junctions besides the artificial metal-base pairing itself. [30,41,46,90] Nevertheless, these systems were based on ligands incorporated internally into the DNA where the metal coordination sum itself to a pre-organized system already established by an environment of neighbouring Watson-Crick base pairing. A complete connection of DNA parts through a solely metal coordination is a recent approach with only a few of attemps until now [30,32,58] and brings a potential to new designs and possible DNA nanoconstructs such as applications, mechanisms and mimicking.

In this project part $\mathbf{3}$ it was developed an artificial nucleotide based on a trans-chelating motif pointing outside of the DNA duplex. The linearity of the group was pursuit to avoid possible distortions on the system producing a metal complex formed through addition of $M(I I)$ perpendicular to the DNA duplex. Because of the properties of the designed ligand, the complexes obtained through coordination with metal species such as $\mathrm{CU}(\mathrm{II})$ and $\mathrm{Pd}(\mathrm{II})$ provide square-planar geometry, giving to the DNA nanoconstruct formed an X shape. The shape of the construct could be used to extend the idea and design here presented and give possibilities of mechanisms where one side of the $X$ construction can be closed (by elongation of the single strands with $T$ bases and formation of metal-base pairing through addition of $\mathrm{Hg}(\mathrm{II})$, for example). The movement reproduced can provide potential mechanisms of opening and closing to develop advanced DNA nanostructures, such as methods to detect and control it.

\subsubsection{Synthesis of the nucleoside}

The goal to produce an interduplex cross-link brings the need of a nucleotide focused in the linearity of the attached motif, to prevent a minimum of distortions and produce, as a product of the complexation, a structure with an $\mathrm{X}$ shape. For this reason the concept of the designed artificial nucleotide demands a ligand perpendicular to the DNA double helix. To combine these features in a single design, it was necessary a backbone with a 5-membered ring fused to a benzene ring, to maintain the straight linear direction for the motif containing two pyridyl arms that will have the role as binding sites for the further metal coordination (see Figure 60). 


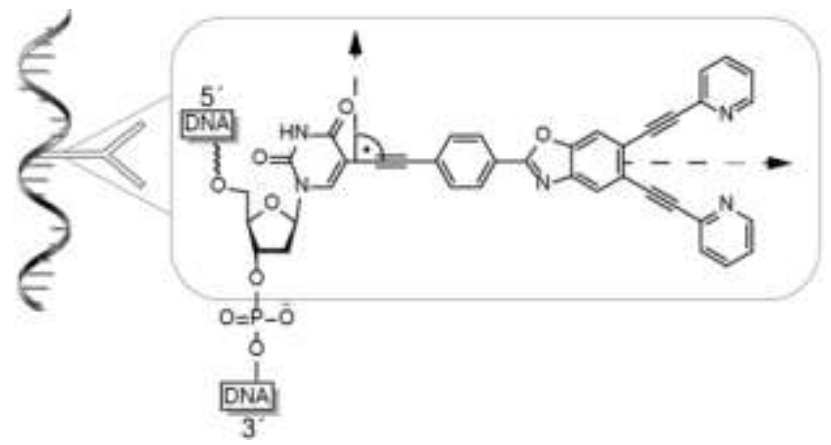

Figure 60 . Scheme for the trans-chelating artificial nucleotide pointing outside of the ss-DNA.

The backbone adopted was chosen based on the possible synthetic steps, within the heterocycle presented as a reasonable choice. Initially it was tried to obtain a backbone based on phthalimide group, but further synthetic steps under basic conditions indicated an instability of the ring to keep the integrity of the ligand structure. An oxazol backbone was assigned as a better choice for a synthetic path and product which attends all the desired features.
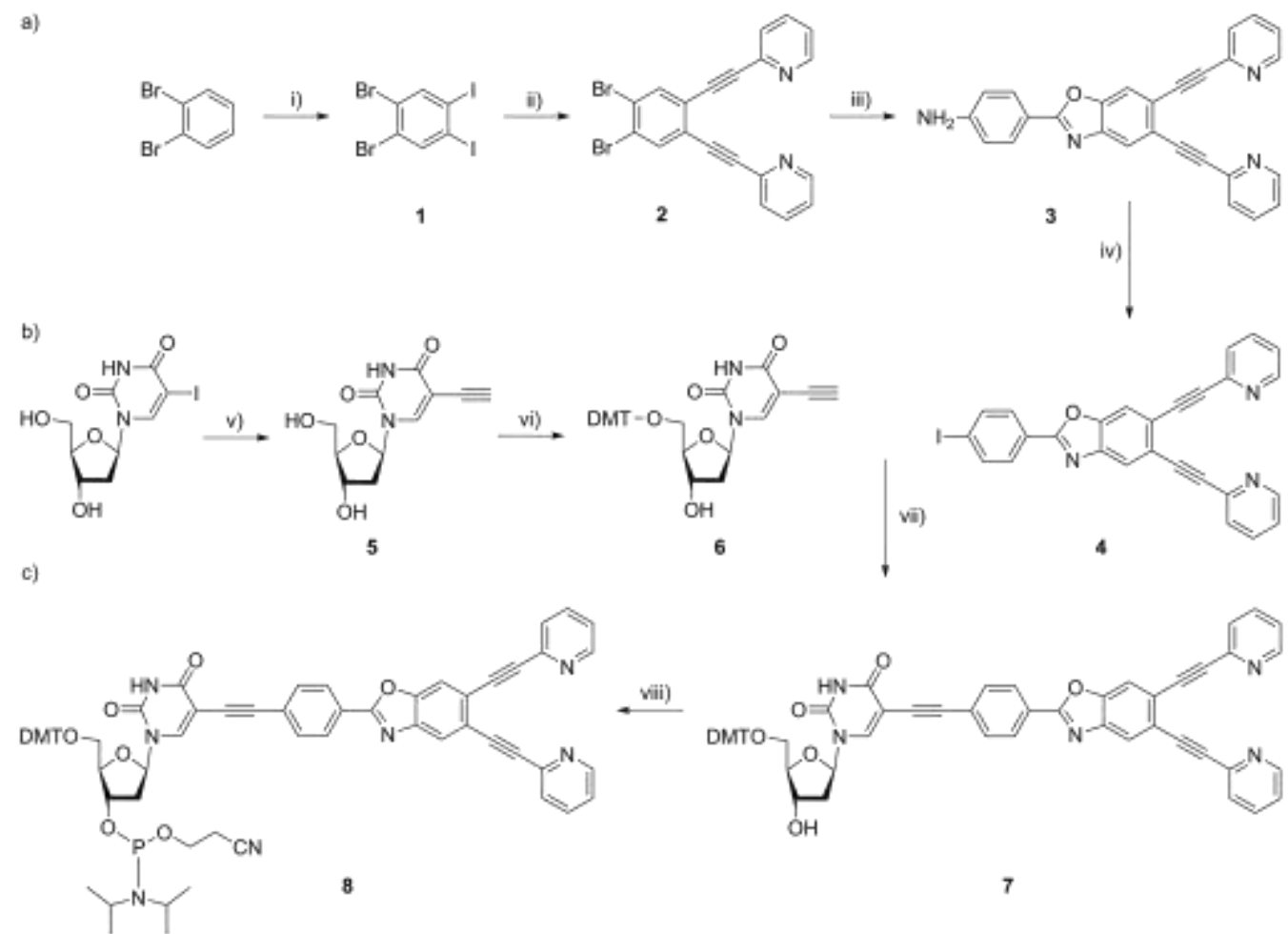

Figure 61. Synthetic path for designed nucleoside 7 and phosphoramidite 8. i) $\mathrm{I}_{2}, \mathrm{H}_{2} \mathrm{SO}_{4}, 40^{\circ} \mathrm{C}$, $1 \mathrm{~h}$; ii) ligand 2, Cul, $\mathrm{PdCl}_{2}\left(\mathrm{PPh}_{3}\right)_{2}, \mathrm{THF}$, 2-ethynyl-pyridine, $\mathrm{Et}_{3} \mathrm{~N}$, r.t., $24 \mathrm{~h}$; iii) $\mathrm{K}_{2} \mathrm{CO}_{3}, \mathrm{Cul}$, toluene, p-amino-benzamide, DMEDA, $120^{\circ} \mathrm{C}, 48 \mathrm{~h}$; iv) i-amyl-ONO, $\mathrm{CH}_{2} \mathrm{I}_{2}, 48 \mathrm{~h}$; vii) ligands 4 and 6, Cul, $\mathrm{PdCl}_{2}\left(\mathrm{PPh}_{3}\right)_{2}, \mathrm{DMF}, 70^{\circ} \mathrm{C}, 16 \mathrm{~h}$; viii) ligand 9, dry THF, NEt(iPr $)_{2}$, $\left(\mathrm{iPr}_{2} \mathrm{~N}\right)\left(\mathrm{OCH}_{2} \mathrm{CH}_{2} \mathrm{CN}\right) \mathrm{PCl}, 2 \mathrm{~h}$. 
A nucleoside containing protected 5'-position with DMT was synthesized to originate the phosphoramidite 8 as building block for the solid phase DNA synthesis. The synthetic path is resumed in eight steps (Figure 61). The deoxyuridine derivative 6 brings a protected 5 'DMT and an ethynyl-group that was attached through Sonogashira cross-coupling between the starting material iodo-2-deoxyuridine and trimethyl-acetylene. Parallel to it, it was synthesized the compound "iodo-oxazol", 4. Further Sonogashira cross-coupling between compounds 4 and 6 gives the desired nucleoside 7 .

Crystal structure was obtained for nucleoside 7 (Figure 62), where it is possible to observe a bend on the DMT group, probably due to the packing conditions which favor the $\pi-\pi$ stacking of the flat aromatic trans-chelating motif. More details concerning the X-ray diffraction can be observed in the experimental part.

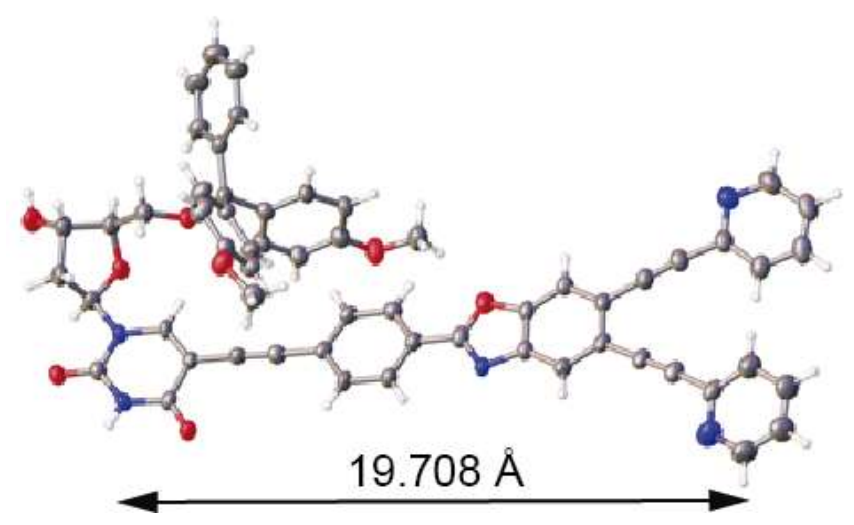

Figure 62. Crystal structure for nucleoside 7. Distance between the $1^{\prime}-\mathrm{N}$-uridine until the binding site was highlighted in the figure.

\subsubsection{Incorporation into the oligonucleotide}

a) Strategy for modified sequences:

The first strategy for an inter cross-link between duplexes is based on the synthesis of the 15 mer $\mathbf{s 3}$, containing a single incorporation of trans-chelating nucleotide right in the middle of the sequence. The oligonucleotide sequence adopted was chosen from the literature ${ }^{[55,91]}$ and it is the same sequence used for the synthesized strand $\mathbf{s} \mathbf{1}$ described in part $\mathbf{2}$. Here the incorporated modification is based in a deoxyuridine residue, with the trans-chelating motif designed to point outside of the duplex s3.s2* formed upon addition of the unmodified complementary strand s2* (Figure 63). 

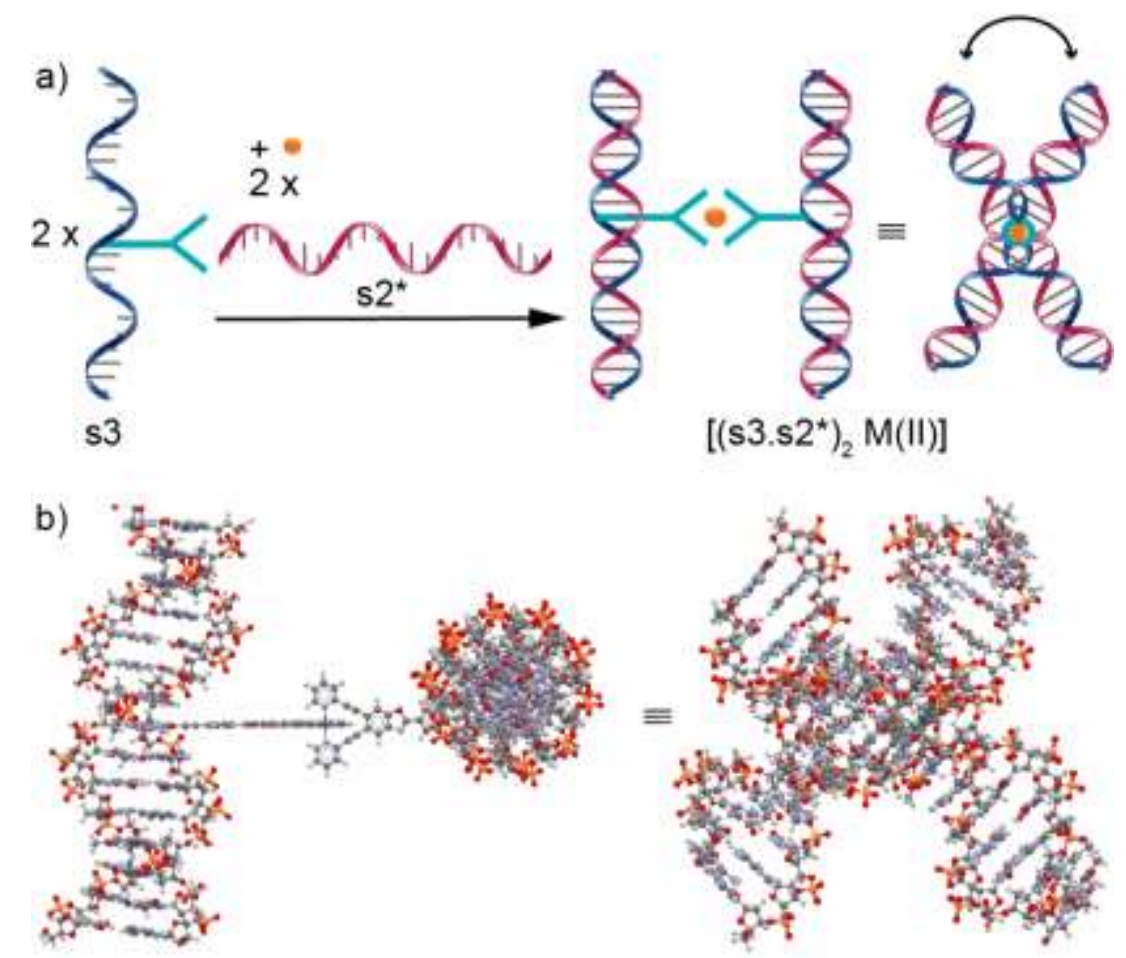

Figure 63. a) Schematic view for the artificial s3 strand and interduplex cross-link for s3.s2*M(II). b) Rough MMFF model made with Spartan software for the intercross linked nanoconstruct. ${ }^{[87]}$

After the formation of the duplex s3.s2* it was added $M(I I)$ solutions (ratio $0.5: 1.0$ of metal for DNA, respectively) to coordinates with two modified nucleotides and lead, through the square-planar complex formed, the formation of an interduplex cross-link made by two duplexes s3.s2*. The nanoconstruct produced by this complexation has a final shape as an $\mathrm{X}$, given by the square-planar geometry from the complex formed (Figure 63b).

The second approach follows the same principle, but in this case the duplex formed contains two trans-chelating nucleotides. The idea of it is basically to increase, through the formation of two complexes, the capability of such inter cross-link formation and its support in hold both duplexes inter connected against repulsive forces arised by the sugar-phosphate backbone due to the proximity of the duplexes. To avoid a possible lower yield caused by coupling of two artificial phosphoramidites $\mathbf{8}$ at a same single strand, the system was designed to obtain a single modification incorporated to each complentary strand, producing $\mathbf{s} 4$ and $\mathbf{s 5}$ that yields the doubly modified duplex s4.s5. It is known that one loop in the DNA duplex corresponds to around 10.4 base pairs; to make sure that both trans-chelating nucleotides are pointing outside of the duplex in the same direction, it was performed a simple molecular mechanics modeling. The modeling with modifications placed under a distance of 10 and 11 base pairing were done and it was concluded as a better result the possibility within a space of 10 base 
pairs. A 15 mer oligonucleotide would be too short for a $10 \mathrm{bp}$ space between the artificial nucleotides (without left them nearly at the ends of the helix). To avoid it, for double modified system it was adopted 21 mers (chosen based on the literature). ${ }^{\text {[91] }}$

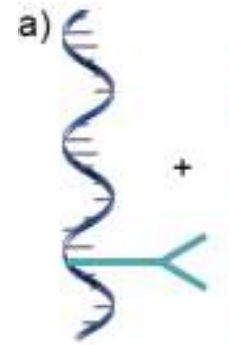

s4

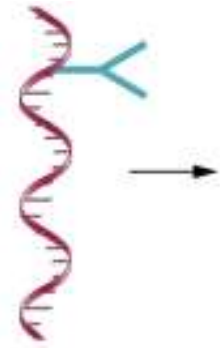

s5

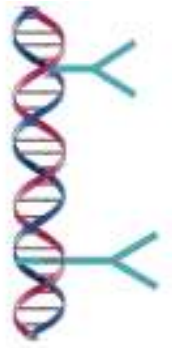

s4.s5

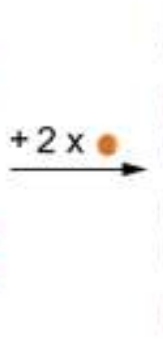

c) b)

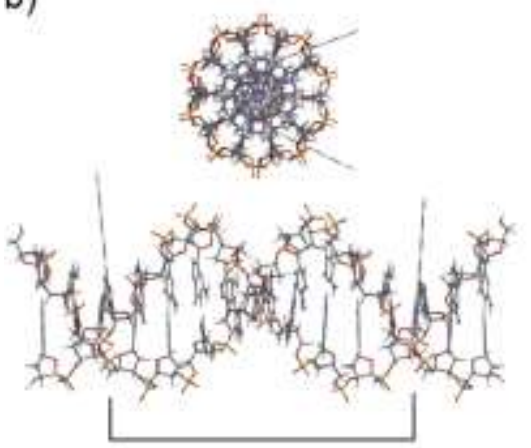

11 bp distance

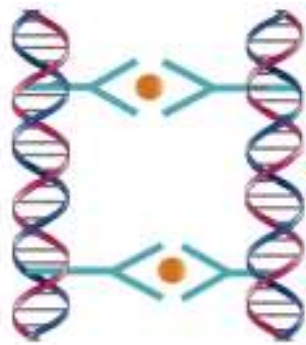

$\left[(\mathrm{s} 4 . \mathrm{s} 5)_{2} \mathrm{M}(\mathrm{II})_{2}\right]$

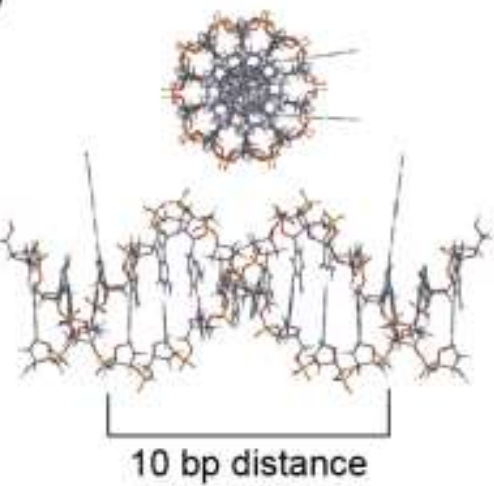

Figure 64. a) Schematic picture of the modified complementary strands s4 and s5 giving the doubly modified duplex s4.s5 and further DNA nanoconstruct upon addition of M(II). Rough MMFF model made with Spartan software for b) 11 base pairing of distance and c) 10 base pairing of distance between the artificial nucleotides. ${ }^{[87]}$

The last strategy adopted was a single strand $\mathbf{s} 6$ containing two trans-chelating nucleotides. The sequence chosen was the same used for the strand s4. For this system, it is possible to evaluate the competition between the inter cross-link formation and a single strand ring structure after addition of $\mathrm{M}(\mathrm{II})$. In this case, both ligands at the same strand dimerize between themselves instead of an inter cross-link through metal coordination between transchelating nucleotides of different s6 strands. The evaluation between both systems and preferences triggered under the presence of complementary unmodified single strand s $\mathbf{7}^{*}$ and excess of $\mathrm{M}(\mathrm{II})$ can be also studied in comparison with the system and behavior of s4.s5 and increases the understanding of the possibilities and mechanisms involving such modifications for nanoconstructs. 
b) DNA syntheses

The DNA sequences were synthesizesd using an Applied Biosystems Expedite machine with CPG beads $(500 \AA)$ on a $1 \mu \mathrm{mol}$ scale and a K\&A Synthesizer $\mathrm{H}-8$ on a $1 \mu \mathrm{mol}$. Solutions of protected 0,1 M phosphoramidites in dry acetonitrile. The solution of the modified phosphoramidite $\mathbf{8}$, due to the low solubility in acetonitrile was made with dry THF and dry 1,2-dichloro-ethane, $\left(\mathrm{CH}_{2}\right)_{2} \mathrm{Cl}_{2}$, the latter presented better results based on the trityl monitor, that follows the synthesis process by UV/Vis measurements of the cleaved $4,4^{`}$-dimethoxytrityl cation, with values no lower than $70 \%$ for strands with single coupled modification. Previous to each synthesis it was performed the unmodified equivalent strand, to ensure the proper synthesizer functioning and good conditions for all solutions prepared. The DNA strands were synthesized on DMT-ON mode to allow an easier further purification by RP-HPLC. The strands synthesized are as followed:

Table 4. DNA sequences for the synthesized single strands. $\mathbf{X}$ as artificial nucleotide.

\begin{tabular}{c|c}
\hline ss-DNA synthesized & \multicolumn{1}{|c}{ Nucleotides from 5'to 3' } \\
\hline s3 & 5'- TAC AAC AXT AAT GTG -3' \\
s4 & 5'- CGT AGC XAG TTT CGA ATC GTA -3' \\
s5 & 5'- TAC GAX TCG AAA CTA GCT ACG -3' \\
s6 & 5'- CGT AGC XAG TTT CGA XAC GTA-3' \\
\hline
\end{tabular}

c) Deprotection of the oligonucleotides and purification:

The first time the synthesis was performed the crude material was submitted to concentrated ammonium hydroxide solution and due to the high basic conditions the artificial ligand was damaged, according to ESI mass spectrum obtained after the cleavage process and treatment of the sample with Zip Tip ${ }^{\circledR} \mathrm{C}_{18}$ Resin. The material of one column from the next synthesis was then fractionated equally and submitted to different milder conditions of possible solutions for cleavage, to evaluate a better cleavage and deprotection way without cause damages on the modified nucleotide. Each fraction was submitted to HPLC to verify those that produce higher peak intensity (better DNA cleavage) and ESI mass measurements to confirm the integrity of the product. The best condition found was a $3 \mathrm{M}$ ammonium hydroxide solution (50:50 methanol/ water) for $8 \mathrm{~h}$ at $60{ }^{\circ} \mathrm{C}$ in a thermoshaker. The other samples of oligonucleotides were all then submitted to this condition of cleavage and deprotection. After it, the 
supernatants were filtered and reduced by evaporation with a vacuum centrifuge. The concentrations were checked by UV/Vis measurements before further purification with RPHPLC to not saturate the column.

The reversed phase HPLC was used to purify the synthesized oligonucleotides, parameters details are given in the experimental part. The presence of failure (the whole sequence without the artificial nucleotide) strand produced in previous syntheses was tried to be supressed by a longer time of coupling and capping steps programed on the synthesizer. It seems the problem was overcome for the strands s3 and s4 which indicates only a shorter strand as impurity. Failure strand and other short trands remains for sequence s5. The undesired strands and other impurities were separated by HPLC. For strand s6 it was confirmed after ESI mass a mixture of the desired sequence with a failure strand containing a single modification. More two cycles of HPLC were performed for the sequence s6, where the similar mobility between the strands produced a broader peak with difficult sepparation due to overlapping. Several attemps of purification eventually meant to a significant decrease of the material, and still not clean enough. The tries to purify this strand were then interrupted. A better approach for purification would be the solid phase synthesis of the strand $\mathbf{s} 6$ with a phosphoramidite containing a fluorous tag, which could give to the desired strand a fluoro affinity and a easier process to sepparate it from the failure strand by using a fluorous column. Another possibility could be the purification through gel electrophoresis. Such possibilities were not possible due to the lack of nucleoside $\mathbf{7}$ to produce more phosphoramidite 8 for more DNA synthesis and the time dependence to produce it. 


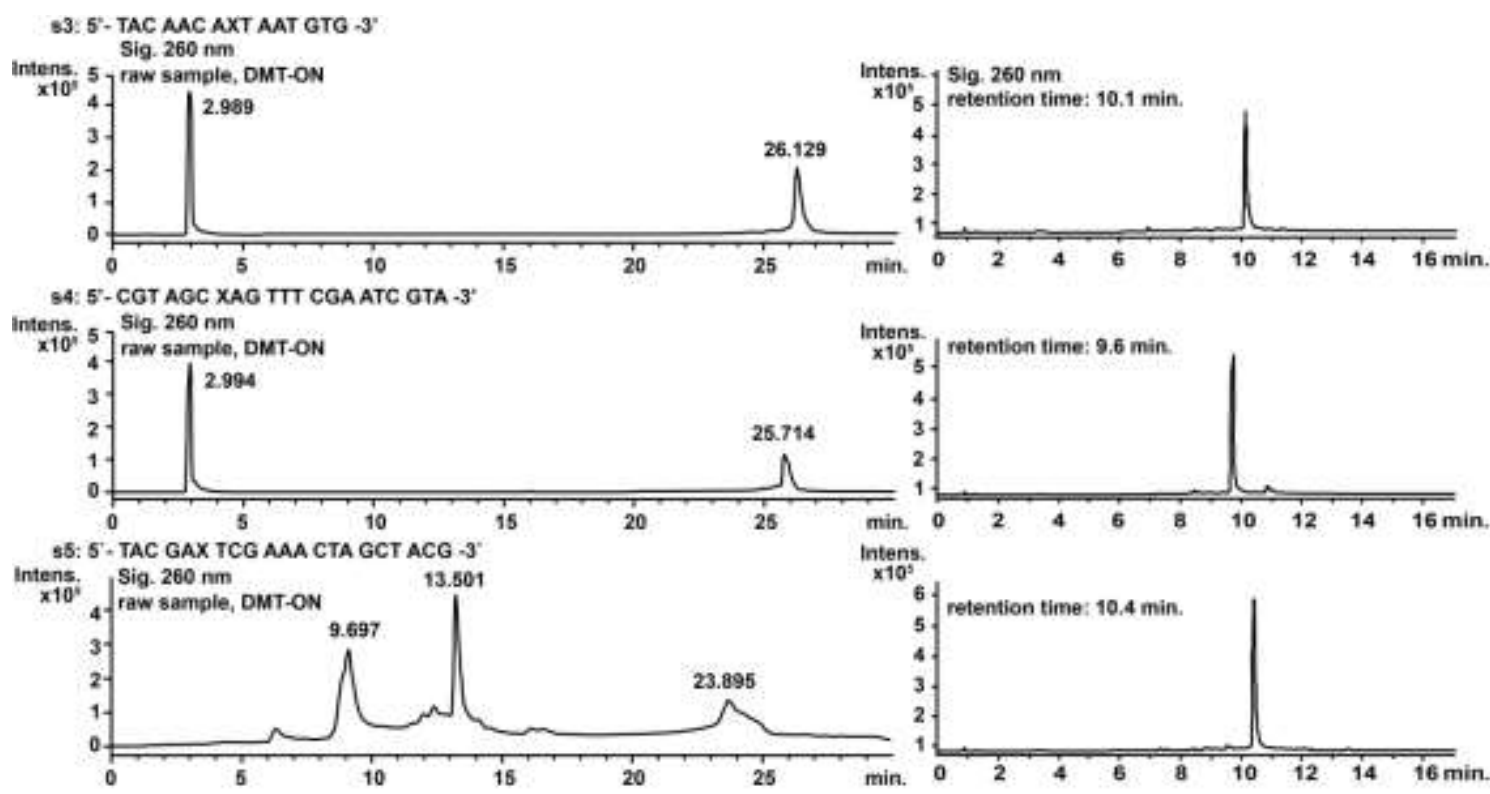

Figure 65. HPLC chromatograms for s3, s4 and s5 strands, respectively (left side as crude materials and right side after purification and detritylation).

Chromatograms for s3, s4 and s5 sequences can be observed in Figure 65. After HPLC purification the strands were reduced under vacuum centrifuge and detritylated/desalted using Glen-Pak ${ }^{\mathrm{TM}}$ cartridges by Glen Research under the fabricant's procedure. The final volumn of the DNA samples were fixed as $1 \mathrm{~mL}$ (and sepparated in two batches) and the concentration determinated by UV/Vis measurements.

d) Determination of the concentration and ESI-MS characterization:

To estimate the DNA concentration, it was applied the base composition method, for reasons explained already in part 2. The determination of the extinction coefficient for artificial nucleoside was made by UV/Vis dilution assay of the detritylated ligand 7, from 0.07 to $0.003 \mathrm{mM}$ (in agreement with the Beer-Lambert law) indicating a slope value for linear regression of the $A_{260}$ plotted against the concentrations, correspondent to $\varepsilon_{260}=19.32 \times 10^{3} \mathrm{~L}$ $\mathrm{mol}^{-1} \mathrm{~cm}^{-1}$. The values used for individual extinction coefficients $\mathrm{A}, \mathrm{T}, \mathrm{G}$ and $\mathrm{C}$ are $15.4,8.7,7.4$ and $11.5 \times 10^{3} \mathrm{~L} \mathrm{~mol}^{-1} \mathrm{~cm}^{-1}$, respectively. ${ }^{[88]}$ The extinction coefficients of the strands are: $\varepsilon_{260}$ $(\mathbf{s} 3)=165.89 \times 10^{3} \mathrm{~L} \mathrm{~mol}^{-1} \mathrm{~cm}^{-1}, \varepsilon_{260}(\mathbf{s} 4)=208.37 \times 10^{3} \mathrm{~L} \mathrm{~mol}^{-1} \mathrm{~cm}^{-1}$ and $\varepsilon_{260}(\mathbf{s 5})=224.12 \times 10^{3} \mathrm{~L}$ $\mathrm{mol}^{-1} \mathrm{~cm}^{-1}$. They were obtained appling the equation for base composition method: $\varepsilon_{260}=\left(\mathrm{n} * \varepsilon_{260} \mathrm{dA}+\mathrm{n} * \varepsilon_{260} \mathrm{dT}+\mathrm{n} * \varepsilon_{260} \mathrm{dC}+\mathrm{n} * \varepsilon_{260} \mathrm{dG}+\mathrm{n} * \varepsilon_{260} \mathrm{dX}\right) * 0.9$, where $\mathrm{n}$ is the number of individual nucleotides and $\mathrm{dX}$ for trans-chelating nucleotide. 0.9 is a correction value taken into account because of the base stacking between the bases on the strand. 


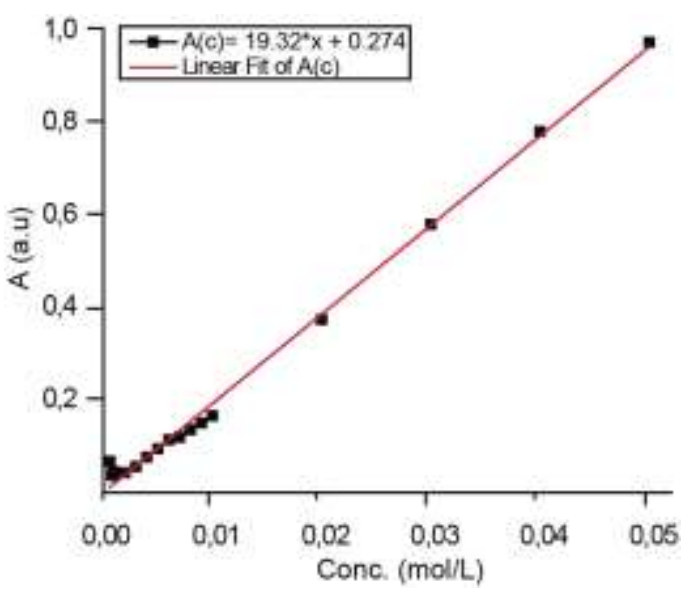

Figure 66. Linear regression of the plotted values of absorption at $260 \mathrm{~nm}$ for detritylated ligand 7 at dilutions from 0.07 to $0.003 \mathrm{mM} \mathrm{CH}_{3} \mathrm{Cl} / \mathrm{MeOH}$ 1:1 solution. Deviations of linearity started after $0.007 \mathrm{mM}$.

ESI-MS spectra for all synthesized oligonucleotides were measured for characterization after proper purification and detritylation/desalting. The whole spectrum for ss-DNA indicates a set of possible negative charges, according to unprotonated possibilities based on the phosphate groups on the backbone. It was possible to observe a clear and good range for $\mathrm{m} / \mathrm{z}-10$ to -2 for all obtained strands, such as adducts containing $\mathrm{Na}^{+}$and $\mathrm{K}^{+}$cations. Slight shifts in the isotopic pattern in comparison to the respective calculation happened due to a not specific calibration of the mass equipment for DNA samples, which could provide a better accurancy of the values. 
a) measured, deconv, -MS
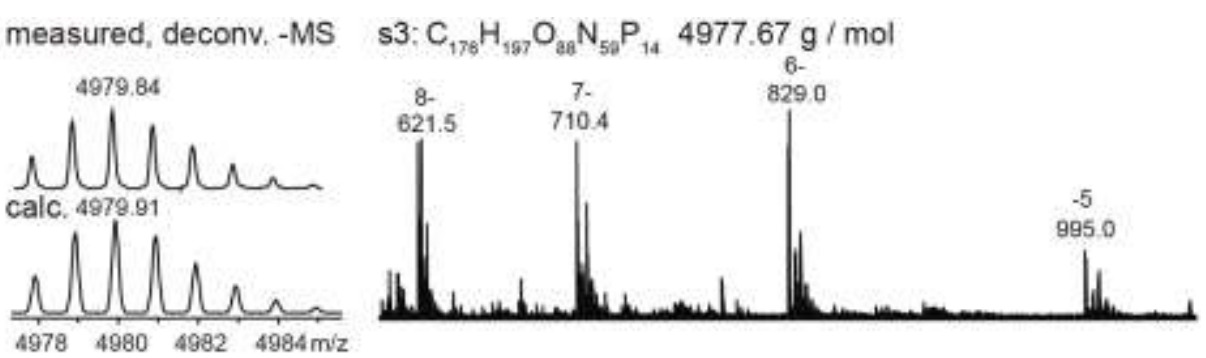

b) measured $[\mathrm{s} 4-10 \mathrm{H}]^{10}$

s4: $\mathrm{C}_{234} \mathrm{H}_{271} \mathrm{O}_{127} \mathrm{~N}_{79} \mathrm{P}_{20} 6837.84 \mathrm{~g} / \mathrm{mol}$

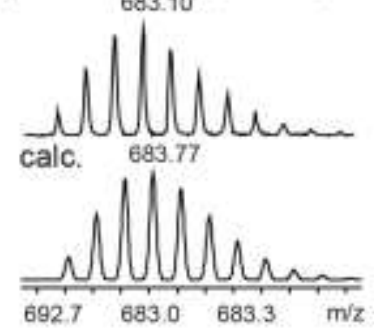

$11-$

$620.9 \quad 10$ -

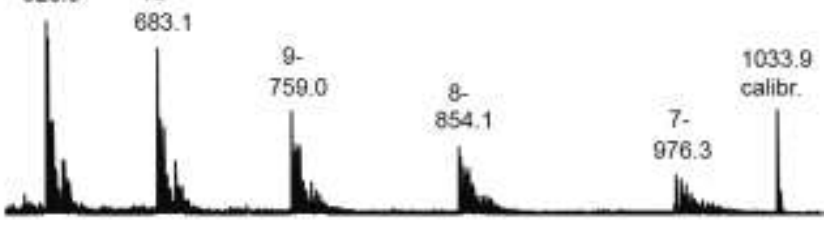

c) measured $[\mathrm{s} 5-10 \mathrm{H}]^{-10}$

s5: $\mathrm{C}_{233} \mathrm{H}_{259} \mathrm{O}_{123} \mathrm{~N}_{83} \mathrm{P}_{20} 6815.86 \mathrm{~g} / \mathrm{mol}$
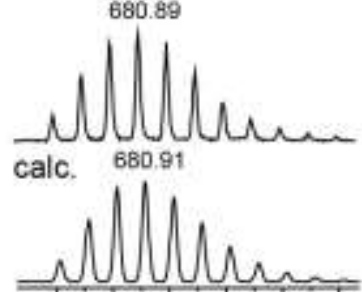

$\begin{array}{lll}680.5 & 680.9 & 681.3 \mathrm{~m} / \mathrm{z} 600\end{array}$

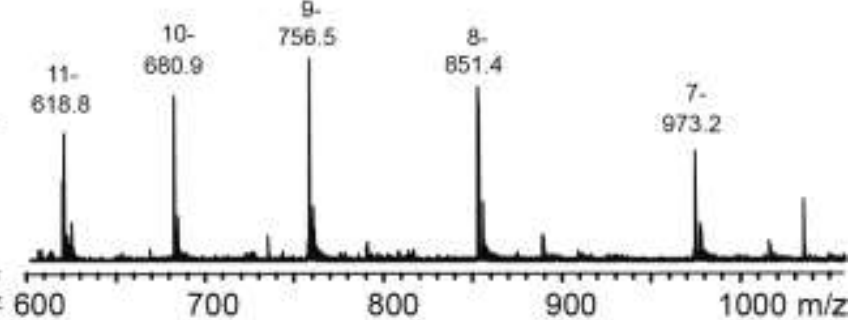

Figure 67. ESI-MS spectra of the characterization for single strands a) s3, b) s4 and c) s5.

\subsubsection{Results for the single modified system, s3.s2*}

\subsubsection{UV/Vis spectroscopy}

UV/Vis spectrum for the strand $\mathbf{s} 3$ indicates the characteristic DNA band (with a maximum of the band at $260 \mathrm{~nm}$ ) related to the stacking between the natural bases. It was observed an additional absorption band in the range of $330-400 \mathrm{~nm}$ attributed to the ligand of the artificial incorporated nucleotide, where such region is consistent to the spectrum for the ligand itself and no band is observed for control measurements performed for analogous unmodified strand $\mathbf{s} \mathbf{1}^{*}$. The UV/Vis spectra for the DNA duplex $\mathbf{s 3 . s 2 *}\left(\mathbf{s} \mathbf{2}^{*}\right.$ is the unmodified single strand with same respective sequence as $\mathbf{s} \mathbf{2}$ containing a nucleobase $\mathrm{A}$ instead of $\mathrm{X}$ ) can be observed with addition of $\mathrm{n}$ equivalents of $\mathrm{M}(\mathrm{II})$ with $\mathrm{NaCl}$ and $\mathrm{NaClO}_{4}$ as electrolytes and CHES buffer (Figure 68 and 69 respectively). 


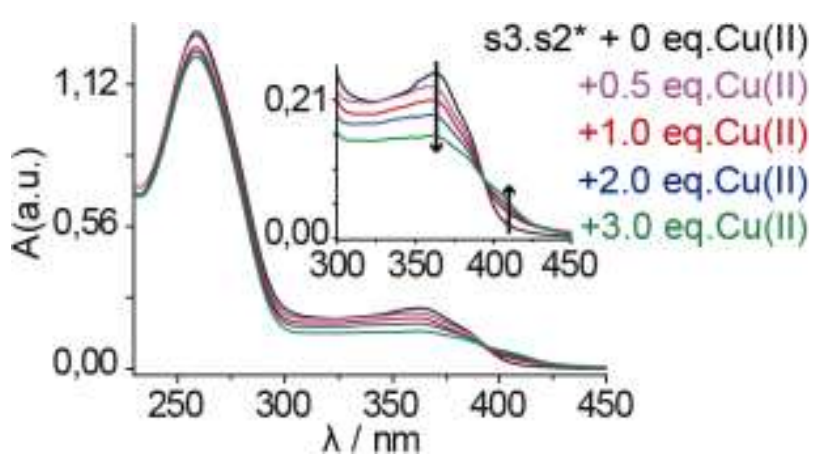

Figure 68. UV/Vis spectra of $3.0 \mu \mathrm{M}$ DNA duplex s3.s2*, $10 \mathrm{mM}$ cacodylate buffer (pH 7.4), $100 \mathrm{mM} \mathrm{NaCl}$ with addition of $\mathrm{n}$ eq. of $\mathrm{Cu}(\mathrm{II})$.

The UV/Vis assay showed above (Figure 68) was performed with $\mathrm{NaCl}$ as electrolyte and can be noticed a decrease of the absorption upon the addition of $\mathrm{Cu}(\mathrm{II})$ at the region attributed to the trans-chelating nucleotide. It could also be observed an isosbestic point at $394 \mathrm{~nm}$ for additions of $\mathrm{Cu}(\mathrm{II})$ higher than 0.5 equivalents and a slight increase of the absorption after this point until wavelength region around $440 \mathrm{~nm}$. The stoichiometric amount to form dimer complex is theorically 0.5 eq. of $\mathrm{M}(\mathrm{II})$, although it seems not be enough to promote metal coordination, evidence that the dissociation constant of the complex formed is low. It was not possible to do a comparison of the profile of UV/Vis spectra between the DNA system and UV/Vis spectra for the complexes made with the ligand $\mathbf{4}$ or the ligand $\mathbf{7}$ (detritylated versions of the ligands) with additions of $\mathrm{Cu}(\mathrm{II})$, due to the insolubility of both ligands after addition of metal $(\mathrm{Pd}(\mathrm{II}), \mathrm{Cu}(\mathrm{II}), \mathrm{Ni}(\mathrm{II}))$. Such comparison of the spectra profiles could help us to determinate if the isosbetic point corresponds to the monomer or dimer complex formed. Nevertheless, additions of $\mathrm{Cu}(\mathrm{II})$ between 0.5 eq. and 1.0 eq. did not indicated another possible crossed point in the spectra, which led us to conclude that even upon excess of 3.0 eq. of $\mathrm{Cu}$ (II) such spectrum profile is related to the formation of the dimer complex. Other data obtained from CD and fluorescence spectroscopy indicated changes in the spectra due to the addition of metal(II), supporting evidence of the metal coordination.

The assays performed under the same conditions, but changing the electrolyte using $\mathrm{NaClO}_{4}$ as electrolyte instead of $\mathrm{NaCl}$, for addition of $\mathrm{Pd}(\mathrm{II})$ (Figure 69a) presented a similar profile. It was observed a decrease of the absorption at the ligand region. But for assays performed with $\mathrm{NaClO}_{4}$ (for $\mathrm{Pd}(\mathrm{II})$ and also for $\mathrm{Cu}(\mathrm{II})$ ) it was possible to notice that such decrease of the absorption is more abrupt and happened to additions of metal higher than 0.5 equivalents. The abrupt decrease of the absorption for the ligand region indicates be related to the electrolyte $\mathrm{NaClO}_{4}$, independent of the $\mathrm{M}(\mathrm{II})$ added and DNA system applied, since the same behaviour was also noticed for the other systems prepared (MBP s1.s2 + Cu(II) presented in 
part 2 and the doubly modified system s4.s5 $+M(I I)$ presented in the section 2.3.4.1). It is not clear how the electrolyte can affect the coordination between the metal cation and influence the binding site environment to produce such behaviour.
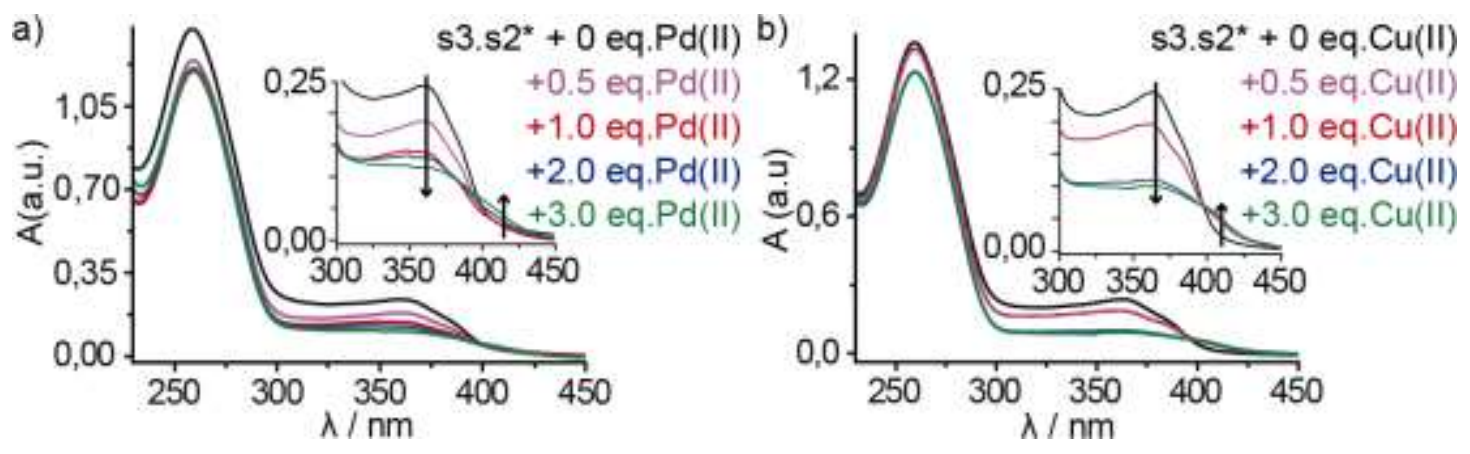

Figure 69. UV/Vis spectra of $3.0 \mu \mathrm{M}$ DNA duplex s3.s2*, $10 \mathrm{mM}$ cacodylate buffer $(\mathrm{pH} 7.4)$, $100 \mathrm{mM} \mathrm{NaClO}_{4}$ with addition of $n$ eq. of $M(I I)$, a) $M(I I)=P d(I I)$ and b) $M(I I)=C u(I I)$.

Despite the abrupt drop in the absorption for additions of $\mathrm{Cu}(\mathrm{II})$ with $\mathrm{NaClO}_{4}$, the final profile is similar to the assay done with $\mathrm{NaCl}$ (Figure 67). The analogous system done upon addition of $\mathrm{Pd}$ (II) (Figure 69a) indicates a difference concerning the appearance of the isosbestic point $(402 \mathrm{~nm})$. For additions of $\mathrm{Pd}(\mathrm{II})$ the isosbestic point only appeared upon addition of 2.0 equivalents with the existence of a second isosbestic point $(385 \mathrm{~nm})$ between the spectra of 2.0 and 3.0 equivalents, which are evidences of two different species formed for each amount of palladium(II) added.

\subsubsection{Thermal analysis}

The thermal analysis indicated that the modified DNA duplex $\mathbf{s 3 . s 2 *}$ is less stable than the unmodified analogous DNA duplex s1*.s2* (nucleobase T instead of $\mathbf{X}$ for $\mathbf{s} \mathbf{1}^{*}$ and nucleobase $A$ instead of $X$ for the strand $\left.\mathbf{s} 2^{*}\right)$ based on the lower value of melting point $\left(\Delta T_{m}=-9,7^{\circ} \mathrm{C}\right)$ obtained (Figure 70a). Both modified DNA duplexes, s3.s2* and s1.s2 (part 2), have a lack of the Watson-Crick base pairing right in the middle of the helical structure, which did not lead a great loss in the stabilibity for the duplex s1.s2 as shown in the results of the part 2. But the trans-chelating ligand incorporated into the DNA s1.s2 were well designed to be flat enough and have a good engagement in the $\pi$-stacking with the adjacent canonical base pairs in the helical shape, such as following the helical structure of the duplex. This indicates that such greater instability in the melting point for the system $\mathbf{s 3 . s 2 *}$ is related to the interactions and distortions caused by the trans-chelating motif pointing outside of the duplex in a single side of the DNA helical structure. 
The instability generated by the outside pointing motif in the B-DNA is not significantly after coordination of the trans-chelating nucleotide with $\mathrm{M}(\mathrm{II})$, whatever complex (monomer $[\mathbf{s 3 . s 2 *} \mathrm{M}(\mathrm{II})]$ or dimer $\left[(\mathbf{s 3 . s 2 *})_{2} \mathrm{M}(\mathrm{II})\right]$ is formed. The low variation in the melting point after the addition of $M(I I)$ was in fact assumed since that the complex formed is outside of the double helix. The melting point and the transition profile for the melting curve of the system s3.s2* $+\mathrm{n}$ eq. of $\mathrm{Cu}(\mathrm{II})$ (with $\mathrm{NaCl}$, Figure $70 \mathrm{~b}$ ) showed greater changes than assays for additions of $\mathrm{Cu}(\mathrm{II})$ and $\mathrm{Pd}(\mathrm{II})$ performed in $\mathrm{NaClO}_{4}$ (Figure 70), which indicates a broader transition for curves of each addition of $\mathrm{Cu}$ (II) (1 eq. to 3 eq.) followed by a slight progressive decrease in the value of the melting point.
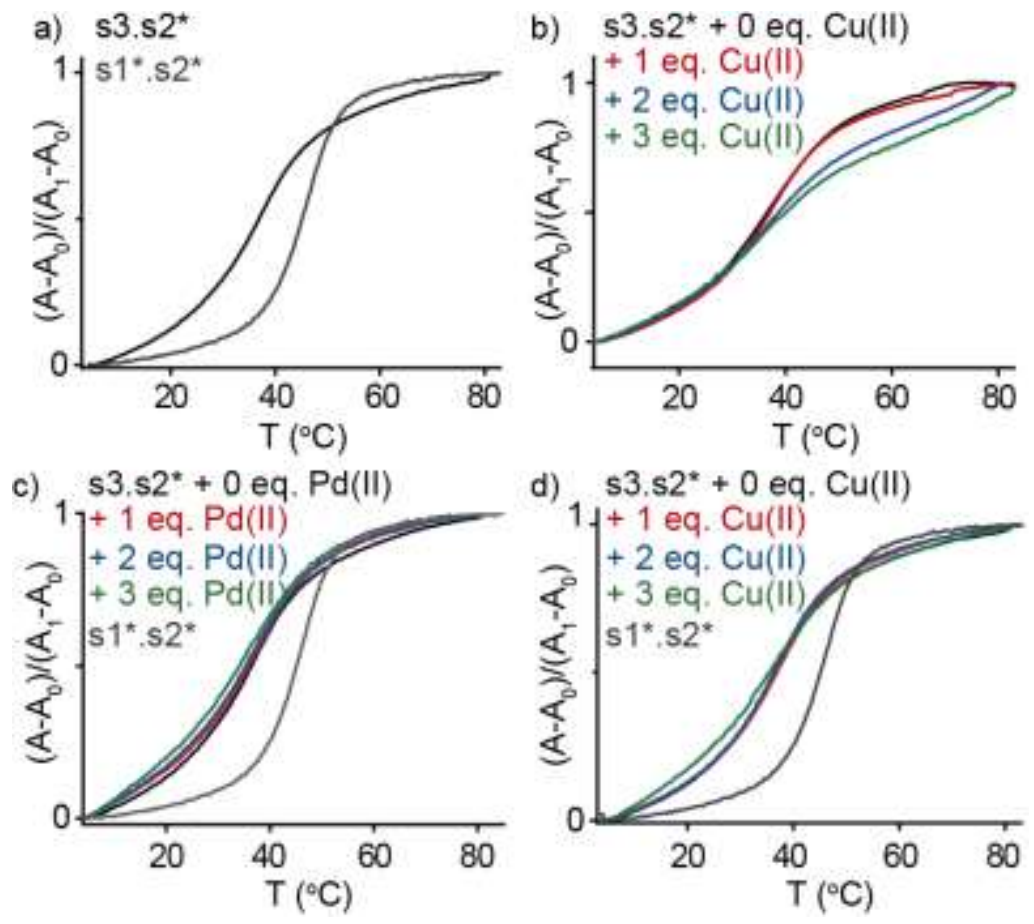

Figure 70. Melting curves for $3.0 \mu \mathrm{M}$ of DNA duplex s3.s2*, $10 \mathrm{mM}$ cacodylate buffer (pH 7.4) a) in comparison to unmodified $\mathbf{s} \mathbf{1} \mathbf{s} \mathbf{2} *$ duplex, both in salinic solution of $100 \mathrm{mM} \mathrm{NaCl}$ and b) $+n$ additions of $\mathrm{Cu}(\mathrm{II})$ and melting curve profiles with $100 \mathrm{mM} \mathrm{NaClO}_{4}$ as electrolyte for the duplex s3.s2* with addition of c) $\mathrm{Pd}(\mathrm{II})$ and d) $\mathrm{Cu}(\mathrm{II})$. All the measurements with temperature rate of $0.5^{\circ} \mathrm{C} / \mathrm{min}$

Table 5. Melting point values for duplex s3.s2* upon addition of $M(I I)$.

\begin{tabular}{c|c|c|c|c|c|c|c}
\hline electrolyte & $\begin{array}{c}\mathbf{s 3 . s 2 *} \\
\mathbf{T m}\left({ }^{\circ} \mathbf{C}\right)\end{array}$ & $\begin{array}{c}\mathbf{s 1 * . s 2 *} \\
\mathbf{T m}\left({ }^{\circ} \mathbf{C}\right)\end{array}$ & $\mathbf{\Delta T m}\left({ }^{\circ} \mathbf{C}\right)$ & $\begin{array}{c}\mathbf{M}(I I) \text { in } \\
\mathbf{s 3 . s 2 *}\end{array}$ & $\begin{array}{c}\mathbf{T m}\left({ }^{\circ} \mathbf{C}\right) \\
\text { 1 eq. }\end{array}$ & $\begin{array}{c}\mathbf{T m}\left({ }^{\circ} \mathbf{C}\right) \\
\text { 2 eq. }\end{array}$ & $\begin{array}{c}\mathbf{T m}\left({ }^{\circ} \mathbf{C}\right) \\
\text { 3 eq. }\end{array}$ \\
\hline $\mathrm{NaClO}_{4}$ & 37.0 & 46.5 & -9.5 & $\mathrm{Pd}(\mathrm{II})$ & 37.0 & 36.2 & 36.6 \\
$\mathrm{NaClO}_{4}$ & 36.9 & 46.5 & -9.6 & $\mathrm{Cu}(\mathrm{II})$ & 35.8 & 36.3 & 36.0 \\
$\mathrm{NaCl}$ & 36.9 & 46.6 & -9.7 & $\mathrm{Cu}(\mathrm{II})$ & 37.6 & 35.9 & 36.0 \\
\hline
\end{tabular}




\subsubsection{Circular dichroism spectroscopy}

Circular dichroism spectra for both complementary single strands, $\mathbf{s 3}$ and $\mathbf{s 2 *}$ such as the duplex s3.s2* indicated interesting effects concerning the DNA chirality transfer onto the ligand. The system s3.s2*, as already mentioned, is analogous to the duplex s1.s2. The difference between $\mathbf{s} \mathbf{1}$ and $\mathbf{s} \mathbf{3}$ is the kind of ligand incorporated into the oligonucleotide, with s1 containing an internal modification based on trans-chelating ligand connected to other nucleotides through hydroxy-ethoxy linkers, while $\mathbf{s} 3$ was based on a trans-chelating motif attached to a modified deoxyuridine, pointing outside of the double helix. The single strand $\mathbf{s} 2$ is internally modified with trans-chelating ligand such as $\mathbf{s} \mathbf{1}$ and $\mathbf{s} \mathbf{2} *$ is its respective unmodifed strand with a dA instead of the artificial ligand incorporated.

The CD spectra of the strand $\mathbf{~} \mathbf{1}$ has a secondary structure as discussed in part 2, where unspecific interactions between the trans-chelating ligand with some not specified nucleobases produces a hairpin structure. ${ }^{[37]}$ Despite the CD spectroscopy more evidences of the hairpin structure were supported by PAGE electrophoresis and the thermal anaysis. Based on it the $C D$ spectrum for $\mathbf{s} 3$ was also submitted to $C D$ measurements at room temperature and at $70^{\circ} \mathrm{C}$ as a way to investigate similar behavior of chirality transfer (Figure 71a). The ligand itself is achiral but the single strand $\mathbf{s} \mathbf{3}$ at $r$.t. indicated such as $\mathbf{s 1}$, unspecific interactions that partially wrap the sequence, transfering a certain degree of chirality from the DNA onto the ligand. This effect produced a band at the range of $366-408 \mathrm{~nm}$. But differently from the single strand $\mathbf{s} \mathbf{1}$ for metal-base pairing (part 2), the secondary structure of $\mathbf{s} \mathbf{3}$ is not a hairpin, fact that was confirmed by control thermal analysis done for the single strand $\mathbf{s} \mathbf{3}$, under the same conditions than the assay performed for the strand s1 ( $3 \mu \mathrm{M}$ ss-DNA, $10 \mathrm{mM}$ cacolylate buffer, $100 \mathrm{mM} \mathrm{NaCl}$ ) and did not indicate an intramolecular equilibrium over different concentrations.

The spectra of $\mathbf{s} 3$ at $70^{\circ} \mathrm{C}$ showed also that an unwinding of the strand interrupts the interactions responsible for the chirality transfer, been observed the vanishing of the band correspondent to the ligand's effect. After allowing the formation of the double helix upon addition of equimolar amount of complementary strand s2*, the formed duplex s3.s2*, differently from s1.s2, did not indicate chirality transfer anymore. This distinct behavior occurs because in this case the system $\mathbf{s 3 . \mathbf { s }} \mathbf{2}^{*}$ has lower chirality transfer than $\mathbf{s 1 . s 2}$, where the ligand is part of the double helix composition. The formation of the duplex s3.s2* prevents to occur the interactions between the nucleobases in the DNA duplex with the trans-chelating motif, that is pointing outside of the duplex, far enough to interact producing high chirality transfer. 

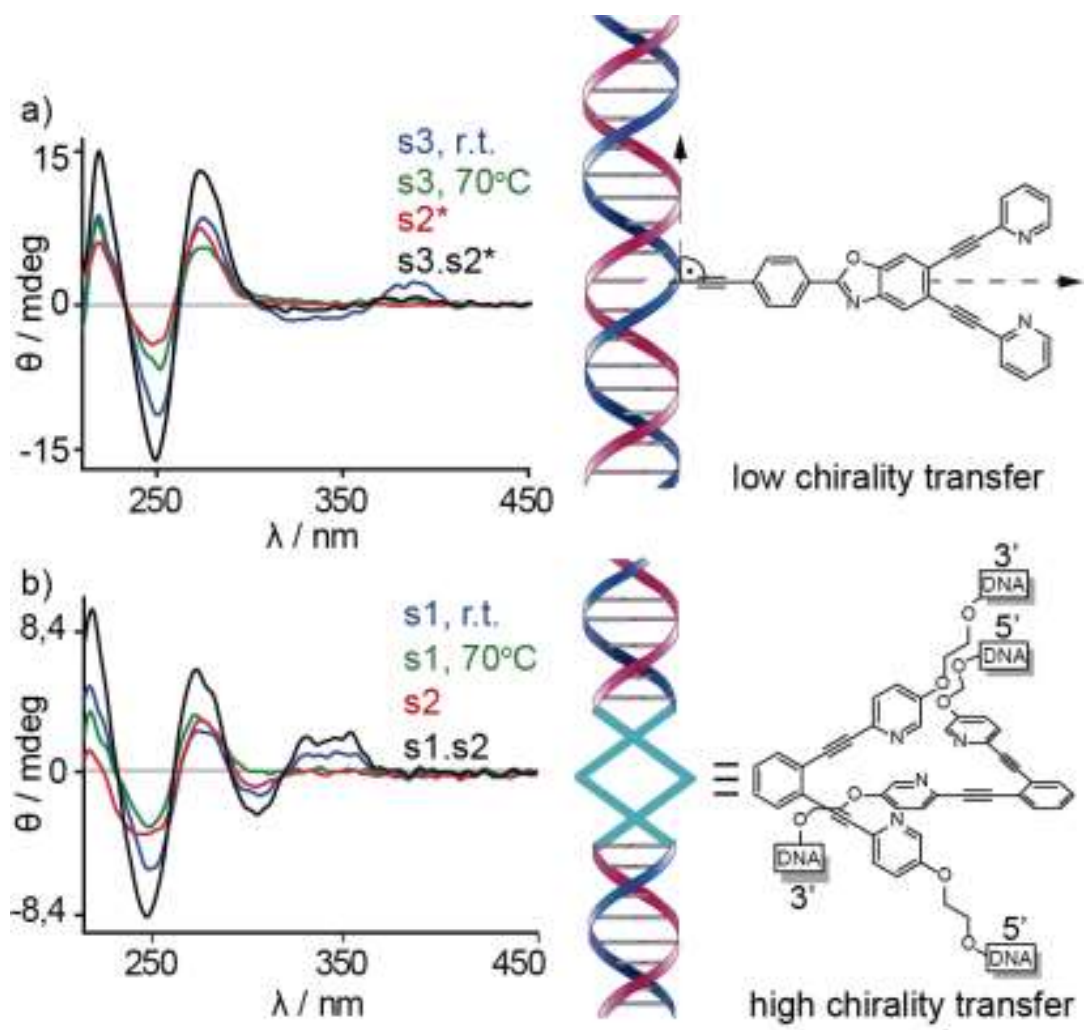

Figure 71. Comparison between the CD spectra and schematic squeme of the modified strands $\mathbf{s} \mathbf{1}$ and $\mathbf{s} \mathbf{3}$ containing incorporation of a trans-chelating ligand a) pointing outside of DNA and b) incorporated internally to the double helix. $3 \mu \mathrm{M}$ ss-DNA and ds-DNA, $10 \mathrm{mM}$ cacolylate buffer (pH 7.4) and $100 \mathrm{mM} \mathrm{NaCl}$.

After formation of the duplex s3.s2*, the intensity of the bands increases as expected due to $\pi$ - stacking interactions on the double helix (Figure 72a). The addition of $\mathrm{Cu}(\mathrm{II})$ on the duplex does not cause significative changes (Figure $72 \mathrm{~b}$ ), with a small decrease in the negative band at $250 \mathrm{~nm}$. The analogous system made with $\mathrm{NaClO}_{4}$ as electrolyte (Figure 73) shows similar behavior for the single strands $\mathbf{s} \mathbf{3}$ and $\mathbf{s} \mathbf{2}^{*}$ and the duplex $\mathbf{s 3 . \mathbf { s }} \mathbf{2}^{*}$, before and after addition of $M(I I)$ (for $\mathrm{Pd}(\mathrm{II})$ and $\mathrm{Cu}(\mathrm{II})$ ).

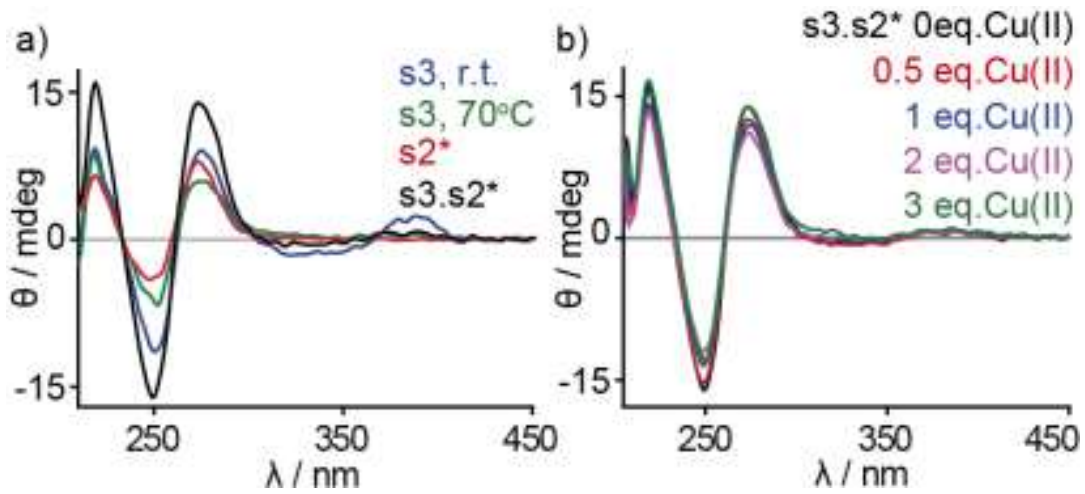

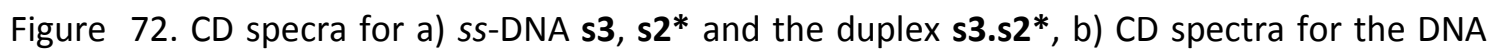
duplex after addition of $\mathrm{Cu}(\mathrm{II})$ equivalents. $3 \mu \mathrm{M}$ ss-DNA and $d s$-DNA, $10 \mathrm{mM}$ cacodylate buffer (pH 7.4), $100 \mathrm{mM} \mathrm{NaCl}$. 

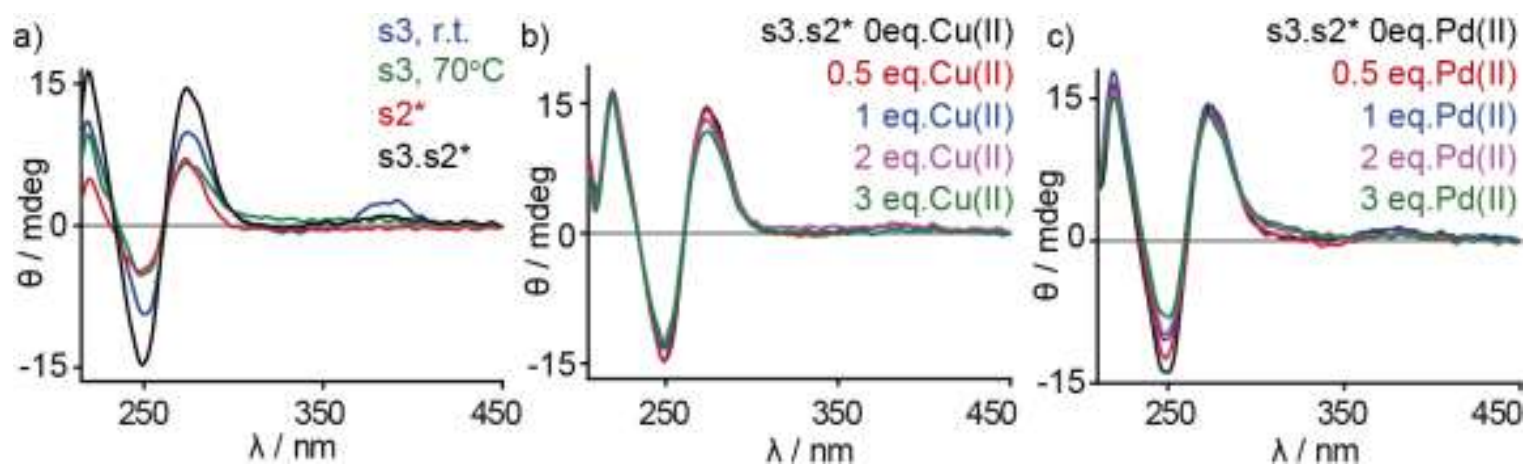

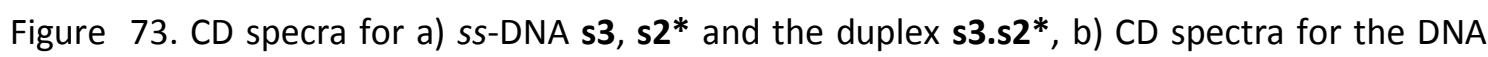
duplex after addition of $\mathrm{Cu}(\mathrm{II})$ and c) after addition of $\mathrm{n}$ eq. of $\mathrm{Pd}(\mathrm{II}) .3 \mu \mathrm{M}$ ss-DNA and ds-DNA, $10 \mathrm{mM}$ cacodylate buffer ( $\mathrm{pH} 7.4), 100 \mathrm{mM} \mathrm{NaClO}_{4}$.

\subsubsection{Fluorescence spectroscopy}

The synthesized single strands and DNA duplexes carrying the artificial trans-chelating nucleotide adopt also its strong fluorescence. The characteristic emission is observed when the sample is excited at $360 \mathrm{~nm}$ (width $2 \mathrm{~nm}$ ) producing emission spectra in a range of 375 $600 \mathrm{~nm}$ with a maximum around $420 \mathrm{~nm}$. The emission spectra for the duplex s3.s2* were measured and demonstrated that upon addition of $M(I I)$, the fluorescence observed is gradually quenched by the $n$ equivalents of $M(I I)$ added. The quenching of the fluorescence is attributed to the metal coordination between the trans-chelating motif and $\mathrm{M}$ (II) (Figure 74). Assays performed in $\mathrm{NaClO}_{4}$ as electrolyte indicated greater quenching efficience of the system for both metals, $\mathrm{Cu}(\mathrm{II})$ and $\mathrm{Pd}(\mathrm{II})$ (Figure $74 \mathrm{~b}$ and c respectively). Nevertheless, it is wortly to remind that greater changes in the system were obtained by thermal analysis for the melting curve of s3.s2* with additions of $\mathrm{Cu}(\mathrm{II})$, especially for the sample using $\mathrm{NaCl}$ as electrolyte.
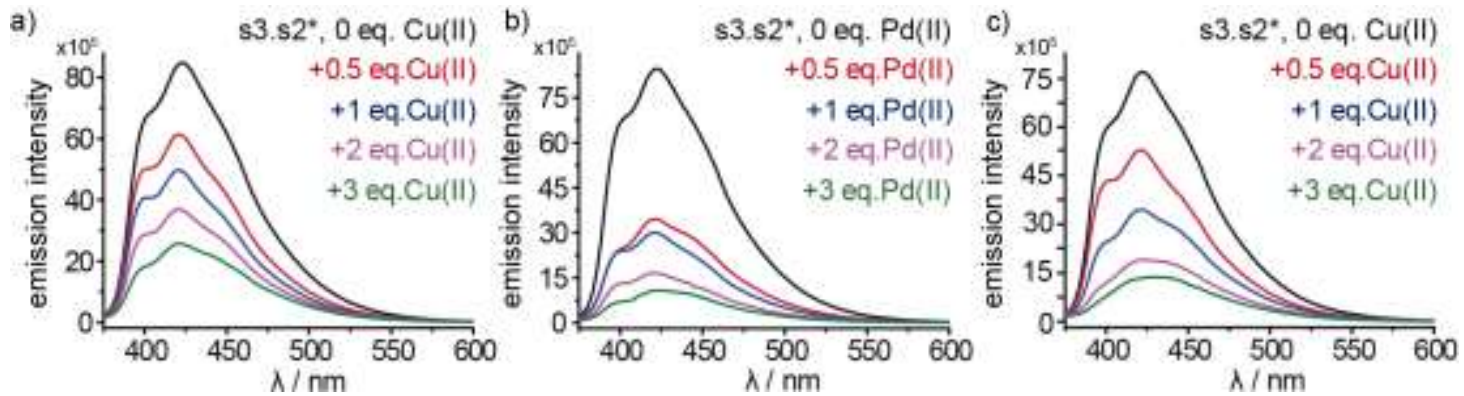

Figure 74. Emission spectra for a) $3 \mu \mathrm{M}$ s3.s2*, $10 \mathrm{mM}$ cacodylate buffer (pH 7.4), $100 \mathrm{mM}$ $\mathrm{NaCl}+\mathrm{n}$ eq. of $\mathrm{Cu}(\mathrm{II})$ and $3 \mu \mathrm{M}$ s3.s2*, $10 \mathrm{mM}$ cacodylate buffer $\left(\mathrm{pH}\right.$ 7.4), $100 \mathrm{mM} \mathrm{NaClO}{ }_{4}+$ $\mathrm{n}$ eq. of $\mathrm{M}(\mathrm{II})$, b) with $\mathrm{M}(\mathrm{II})=\mathrm{Pd}(\mathrm{II})$ and $\mathrm{c}$ ) with $\mathrm{M}(\mathrm{II})=\mathrm{Cu}(\mathrm{II})$. Excitation wavelength at $360 \mathrm{~nm}$, width $2 \mathrm{~nm}$. 
Table 6. Percentage of quenched emission values (maximum) for the duplex s3.s2*, $10 \mathrm{mM}$ cacodylate buffer, $100 \mathrm{mM} \mathrm{NaCl}$ or $\mathrm{NaClO}_{4}$ with different equivalents of $\mathrm{M}(\mathrm{II})$ added. [67]

\begin{tabular}{c|ccccc}
\hline $\mathbf{3} \boldsymbol{\mu M}$ DNA & M (II) & 0.5 eq. & 1 eq. & 2 eq. & 3 eq. \\
\hline $\mathrm{NaCl} 100 \mathrm{mM}$ & $\mathrm{Cu}(\mathrm{II})$ & $27.0 \%$ & $41.0 \%$ & $56.2 \%$ & $69.2 \%$ \\
$\mathrm{NaClO}_{4} 100 \mathrm{mM}$ & $\mathrm{Pd}(\mathrm{II})$ & $59.0 \%$ & $64.3 \%$ & $80.2 \%$ & $87.5 \%$ \\
$\mathrm{NaClO}_{4} 100 \mathrm{mM}$ & $\mathrm{Cu}$ (II) & $30.8 \%$ & $54.7 \%$ & $75.0 \%$ & $82.7 \%$ \\
\hline
\end{tabular}

\subsubsection{Förster resonance energy transfer, FRET}

So far the spectroscopic data obtained by UV/Vis, CD and fluorescence evidenced the metal coordination with the artificial trans-chelating nucleotide upon addition of $M(I I)$, such as slight changes in the stability of the DNA system concluded by thermal analysis. The metal

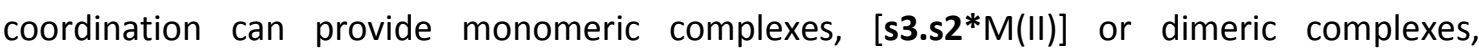
$\left[(\mathbf{s 3 . s 2 *})_{2} \mathrm{M}(\mathrm{II})\right]$. The latter is obtained through interduplex cross-link which is the goal of this part 3. Measurements of Förster resonance energy transfer were performed as a way to provide more details concerning the environment of the DNA nanostructure formed by the metal complexation. The modified single strand $\mathbf{s} \mathbf{3}$ was combined in equimolar amount to its complementary counterstrand $\mathbf{s} \mathbf{2}^{\mathrm{D}}$, carrying a donor-acceptor pair (5'-Hex-sequence-BHQ1-3 $\left.{ }^{\circ}\right)$,

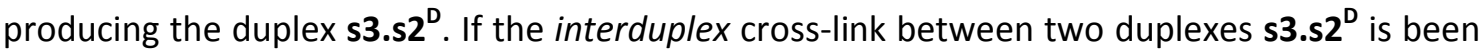
formed through the complexation upon addition of $M(I I)$, the system will be then able to demonstrate an energy-transfer resonance from the donor (Hex) to the acceptor (BHQ1) since the short distance between them (a weak coupling mechanism) produces the quenching of the fluorescence (the acceptor BHQ1 is a non fluorophore molecule). ${ }^{[67,92]}$

This scenery would be possible through the interduplex cross-link, giving two possible intercrossed units (Figure 75), with both acceptors on the same side, facing each other (structure $A$ in Figure 75) or with Hex and BHQ1 in alternated sides (structure B in Figure 75). In the structure A no energy-transfer would occur and the intensity of the fluorescence emitted would be the maximum. For the structure $B$, the energy transfer can occur by the weak coupling due to the distance from the donor to the acceptor, causing a quenching of the fluorescence, providing a signal with zero intensity. Due to the fact that the artificial nucleotide contains the trans-chelating motif attached through an alkyne bridge to the deoxyuridine part, the system is allowed to turn, such spinning might give an averaged value of FRET (from the alternance between the structures $A$ and $B$ ). 


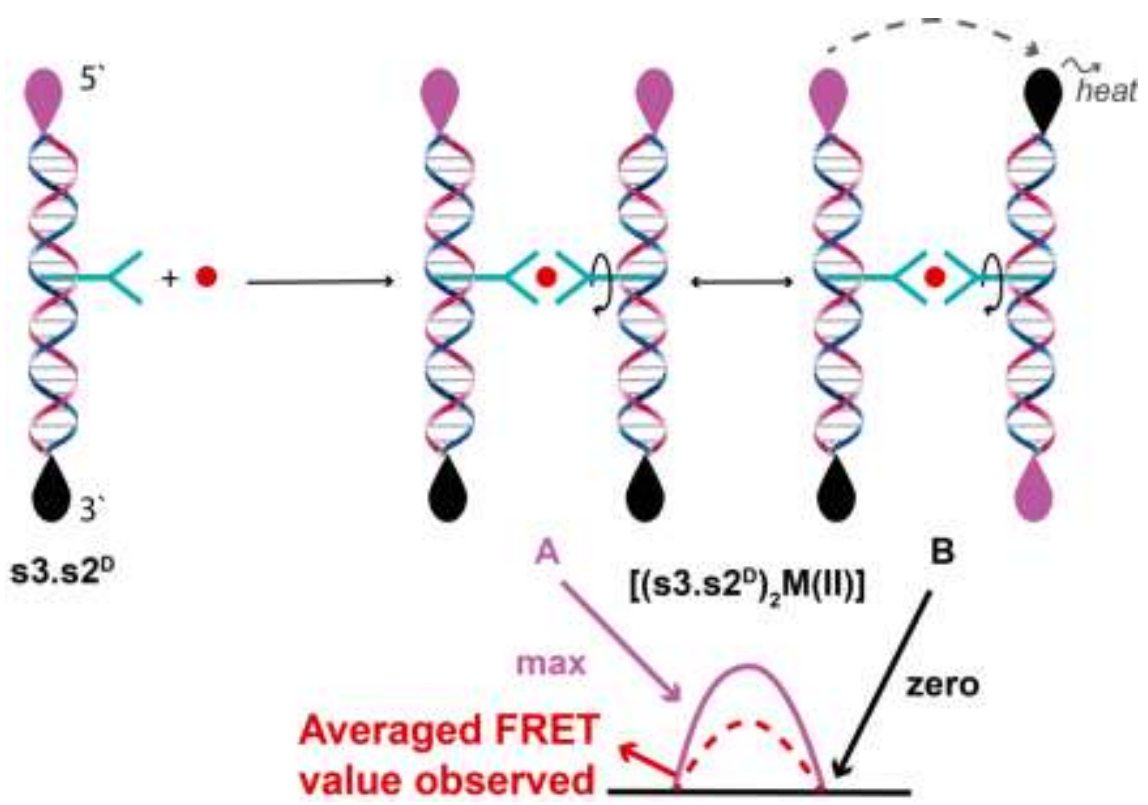

Figure 75. Scheme for inter cross-link between duplexes formed through dimer metal complexation and possible units, $A$ and $B$, with their respective figurative intensities of emission.

The duplex $\mathbf{s} 3 . \mathbf{s} 2^{\mathrm{D}}$ upon further additions of $\mathrm{Cu}(\mathrm{II})$ were measured by fluorescence and also by UV/Vis as a matter of control. Fluorescence and UV/Vis measurements of the single strand $\mathbf{s} \mathbf{2}^{\mathrm{D}}$ were performed to discard possible contact quenching interactions produced by the folding of the single strand (a ground-state complex produced by quencher-donor would be detected by UV/Vis ${ }^{[67]}$ ). The fluorescence measurements were performed with excitation based on the ligand region ( $360 \mathrm{~nm}$ ) with emission range $375-700 \mathrm{~nm}$ (Figure 76) and also with excitation based only in the FRET pair region (excitation of $510 \mathrm{~nm}$ and emission range 525-700 nm) shown in the appendix (Figure 100). The single strand $\mathbf{s} 3$ was also submitted to fluorescence and UV/Vis spectroscopy such as a comparison with the system not carrying the FRET pair, s3.s2*. Surprisingly, the duplex s3.s2* at the ligand region presents an intensity of the emission 30\% higher (maximum at $424 \mathrm{~nm}$ for ligand region) than its analogue duplex $\mathbf{3} 3 . \mathbf{s} \mathbf{2}^{\mathrm{D}}$ carrying the FRET pair Hex/BHQ1 (Figure 76a, lines black and cyan respectively). This indicates that the distance between the artificial nucleotide (placed as $8^{\text {th }}$ nucleotide on the 15 mer $\mathbf{s} 3$ ) and the BHQ1 is valid to produce a weak-coupling interaction between them, with the acceptor quenching partially also the fluorescence of the trans-chelating nucleotide.

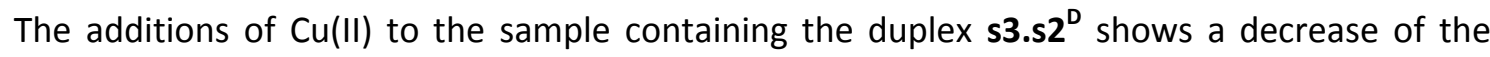
emission intensity for the ligand region (Figure 76a) with similar profile to the one presented in section 2.3.3.4 for s3.s2*. Such effect indicates a quenching of the fluorescence of the ligand 
upon the addition of metal(II), due to the complexation. It is also possible to see in the range of 525-600 $\mathrm{nm}$ the emission intensity attributed to the FRET pair Hex/BHQ1.

a)

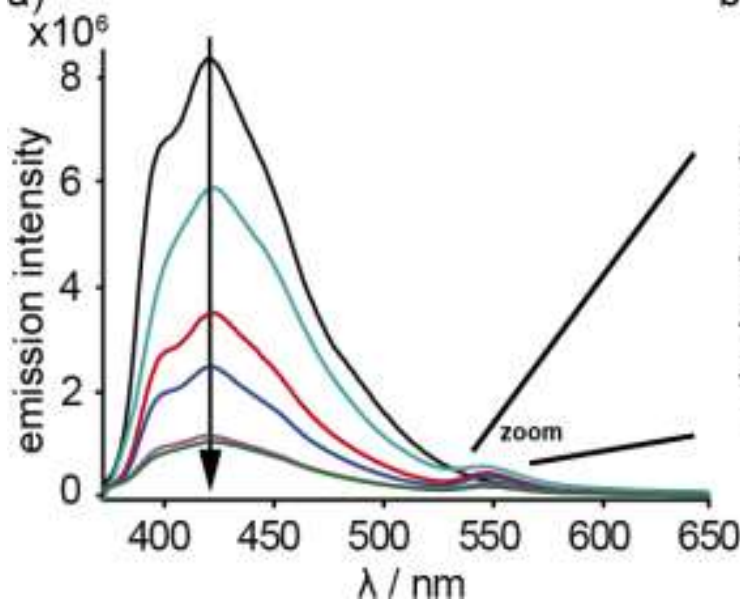

b)

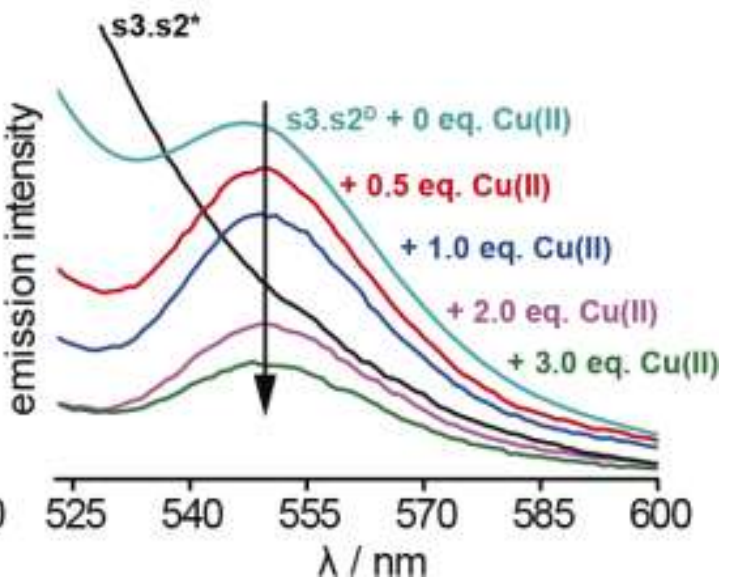

Figure 76. Fluorescence spectra for the DNA duplexes $\mathbf{s 3 . s 2 *}$ and $\mathbf{s 3 . s 2 ^ { \mathrm { D } }}$ and further additions

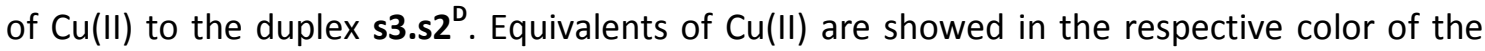
lines in the spectra. a) View of the entire spectra, b) zoom of the range 525-600 $\mathrm{nm}$ related to the range of emission for the FRET pair Hex/BHQ1. Excitation $360 \mathrm{~nm}$, emission range 375$650 \mathrm{~nm}$, width $2 \mathrm{~nm}$. Sample conditions: $3.0 \mu \mathrm{M}$ ds-DNA, $10 \mathrm{mM}$ cacodylate buffer ( $\mathrm{pH}$ 7.4), $100 \mathrm{mM} \mathrm{NaCl}$.

The dimer complex $\left[\left(\mathbf{s 3 . s 2}{ }_{2}^{\mathrm{D}}{ }_{2} \mathrm{Cu}(\mathrm{II})\right]\right.$ will be able to indicates a drop in the emission intensity on the FRET region, as a consequence of the weak coupling formed with energy transfer from the donor fluorophore Hex to the acceptor BHQ1, as indeed can be observed in Figure 76b. Further additions of $\mathrm{Cu}(\mathrm{II})$ ( 4 eq. and 5 eq.) indicated a reversed path, with the emission intensity at the range 525-600 nm slightly increasing again, which we postulate as evidence to the formation of monomer complex [s3.s2 $\left.{ }^{\mathrm{D}} \mathrm{Cu}(\mathrm{II})\right]$ (see in the appendix Figure 100). Additions of copper(II) higher than 3 equivalents in the system s3.s2* measured by thermal analysis were not possible to provide a reasonable profile to lead an interpretation, indicating a system to complex, probably due to the excess of salts in the sample.

\subsubsection{Electrospray ionization mass, ESI-MS spectrometry}

Electrospray ionization mass spectroscopy was performed in negative ion mode to detect metal-DNA adducts. The profile for ESI-MS DNA spectrum, as already explained, indicates isotopic peaks with different charge states related to the amount of $\mathrm{PO}_{4}^{-}$groups presented in the DNA sequence. Due to the conditions of ionization it was difficult to preserve the integrity of the duplex s3.s2* and the efforts were focused to detect a possible inter cross-link between two $\mathbf{s} 3$ single strands through metal complexation. DNA samples in the presence of ammonium 
acetate produced a stronger noise with higher intensity than possible peaks produced for metal-DNA adducts. In the case of samples prepared in the presence of triethylamine, no metallated adducts were detected, such fact indicates a possible "stealing" of the metal promoted by $\mathrm{Et}_{3} \mathrm{~N}$. It was possible to obtain good results for samples in aqueous solution in an equimolar mixture with acetonitrile (50:50). Samples with concentration of $20 \mu \mathrm{M}$ $\left(\mathrm{H}_{2} \mathrm{O} / \mathrm{MeCN}\right)$ were prepared and submitted for measurements (details of the sample preparations and equipment parameters in the experimental part).

Surprisingly it was observed isotopic pattern attributed to a dimeric unmetallated structure formed by two units of the single strand $\mathbf{s 3}$. It is not clear the possible interactions responsible for such adduct, but it is important to remind that the nucleotide sequence of the strand $\mathbf{s} 3$ is the same used for strand $\mathbf{s} \mathbf{1}$ in part $\mathbf{2}$ and literature, in both works such dimeric structure was not obtained. ${ }^{[37,55]}$ We suggest that the dimer structure formed is related to unspecific interactions caused by the artifical nucleotide incorporated into the DNA sequence with an important role of the outside pointing trans-chelating motif of the strand, been possibly due to $\pi-\pi$ stacking between two motifs of different strands. The simulation of the isotopic pattern also shows possible derivative adducts of the dimer structure with sodium and potassium (Figure 77 and 78) for different charges where the most well defined peaks were visualized for $m / z=-13,-12$ and -11 . There is a slight shift of the isotopic pattern measured in comparison to the calculated values. This occurs due to the equipment calibration, performed with "Tuning Mix", a standard solution from Agilent for calibration that was used to calibrate in the positive such as well in the negative ion mode. The values concerning the isotopic peaks could be better accurated if the calibration would be performed specifically for DNA samples.

The peaks attributed to the metallated inter cross-link between two s3 strands were obtained for addition of $\mathrm{Cu}(\mathrm{II})$ and $\mathrm{Ni}(\mathrm{II})$. For nickel(II) was also possible to observe formation of adduct with one cation of sodium (Figure 78b). Control measurements were performed with analogous unmodified strand (s1*) upon addition of $\mathrm{CU}(\mathrm{II})$ and $\mathrm{Ni}(\mathrm{II})$ under the exactly same conditions of sample preparation and measurement parameters and was not indicated any possible metallated-adduct as a product of unspecific binding. It was still possible to observe peak of unmetallated single strand s3 upon addition of $M(I I)$, indicating that the metal coordination was not complete, such as monomeric adducts s3.M(II) (see appendix Figures 102 and 103), possibly products of the broken dimeric structure due to the rush conditions to promote the ionization. 


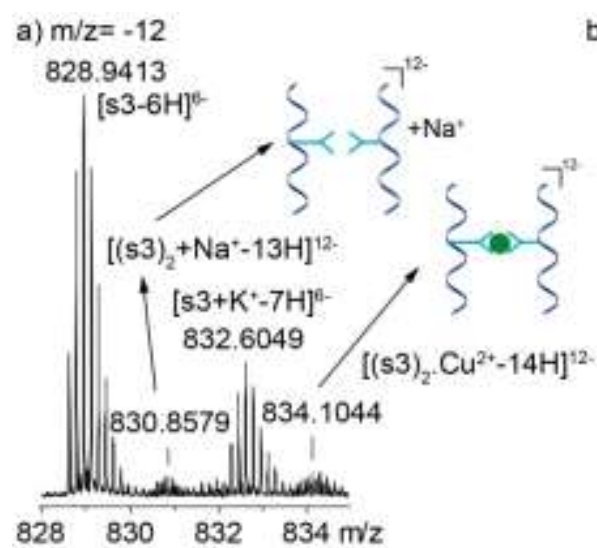

c) measured $\mathrm{m} / \mathrm{z}=-13$

765.0985 b) measured $\left[(\mathrm{s} 3)_{2}, \mathrm{Cu}^{2 *}-13 \mathrm{H}\right]^{1+}, \mathrm{s} 3=\mathrm{C}_{178} \mathrm{H}_{197} \mathrm{O}_{68} \mathrm{~N}_{s 9} \mathrm{P}_{14}$ 910.0223

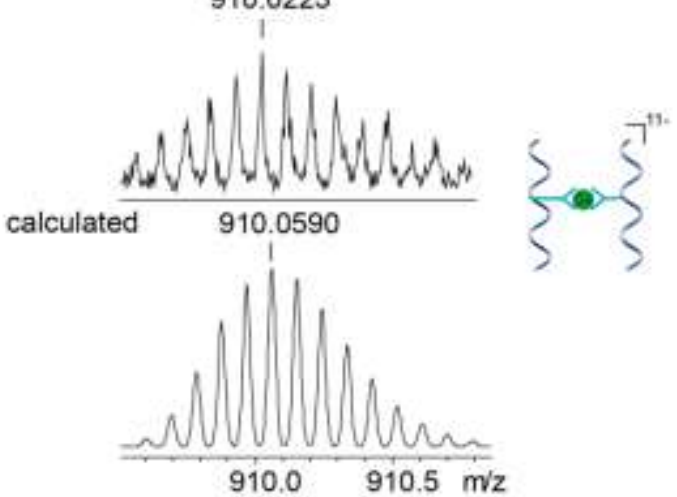

766.8679

768.0994
769.7849

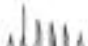

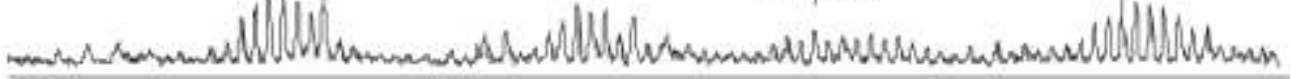
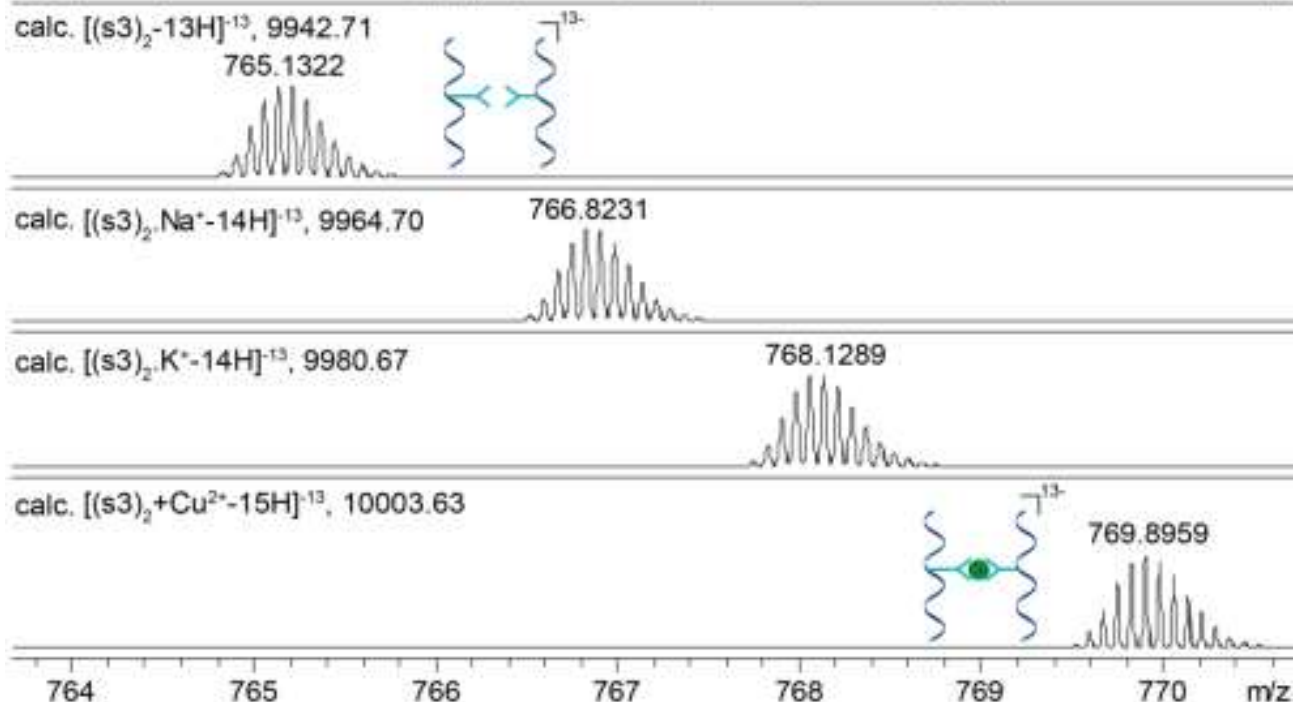

d) measured $\mathrm{m} / \mathrm{z}=-11$

$904.4869 \quad 906.4755$ calc. $\left[(\mathrm{s} 3)_{2}-11 \mathrm{H}^{-11}, 9944.73 \quad 907.8269\right.$ 904.4304

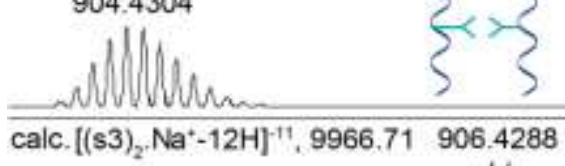
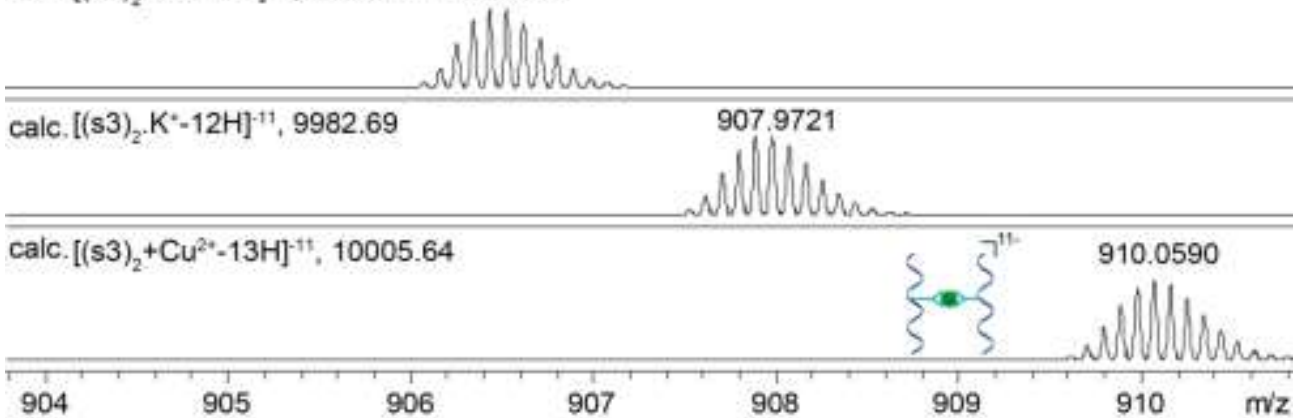

Figure 77. ESI-HRMS of $20 \mu \mathrm{M} s s-D N A$ s3 adducts with $\mathrm{Cu}(\mathrm{II}), \mathrm{H}_{2} \mathrm{O} / \mathrm{MeOH}$ (50:50). a) Unmetallated and metallated dimers $\mathrm{m} / \mathrm{z}=-12$, b) Pattern for $\operatorname{dimer}\left[(\mathrm{s} 3)_{2} \mathrm{Cu}^{2+}-13 \mathrm{H}\right]^{-11}$. Stacking for the spectrum of dimeric adducts c) $m / z=-13$ and d) $m / z=-11$. 
a) measured $m / z=-13, s 3=\mathrm{C}_{178} \mathrm{H}_{197} \mathrm{O}_{68} \mathrm{~N}_{62} \mathrm{P}_{14}$

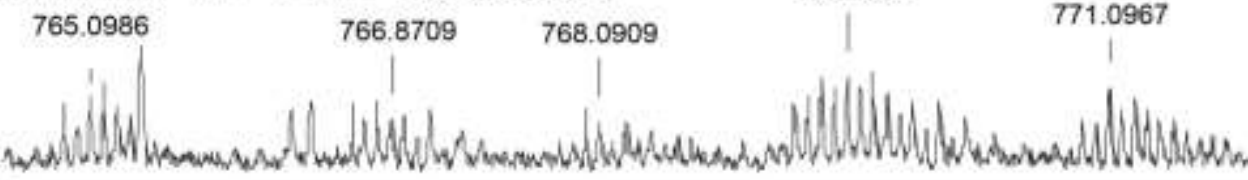

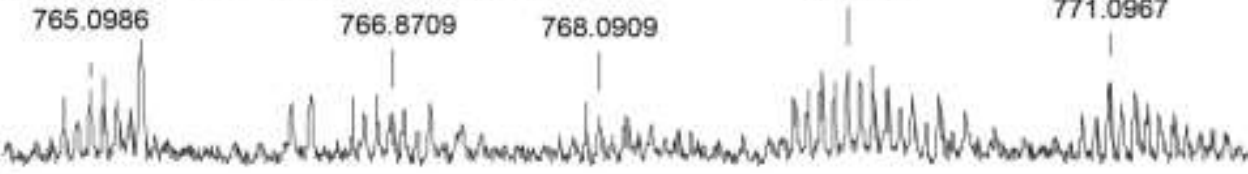

769.5545 calc. $\left[(\mathrm{s} 3)_{2}-13 \mathrm{H}\right]^{-13}, 9942.71$

765.1322

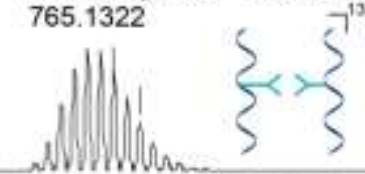

calc. $\left[(\mathrm{s} 3)_{2} \cdot \mathrm{Na} *-14 \mathrm{H}\right]^{13}, 9964.70$

766.9002

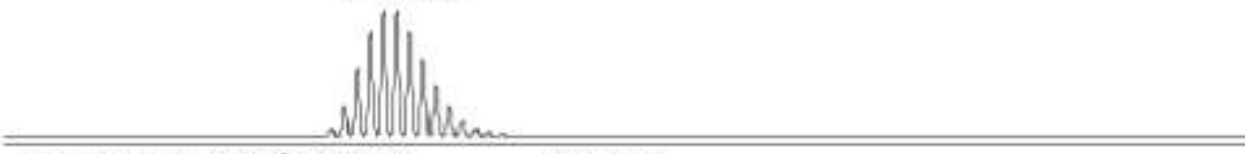

calc. $\left[(\mathrm{s} 3)_{2} \cdot \mathrm{K}-14 \mathrm{H}\right]^{+3}, 9980.67$

768.1289

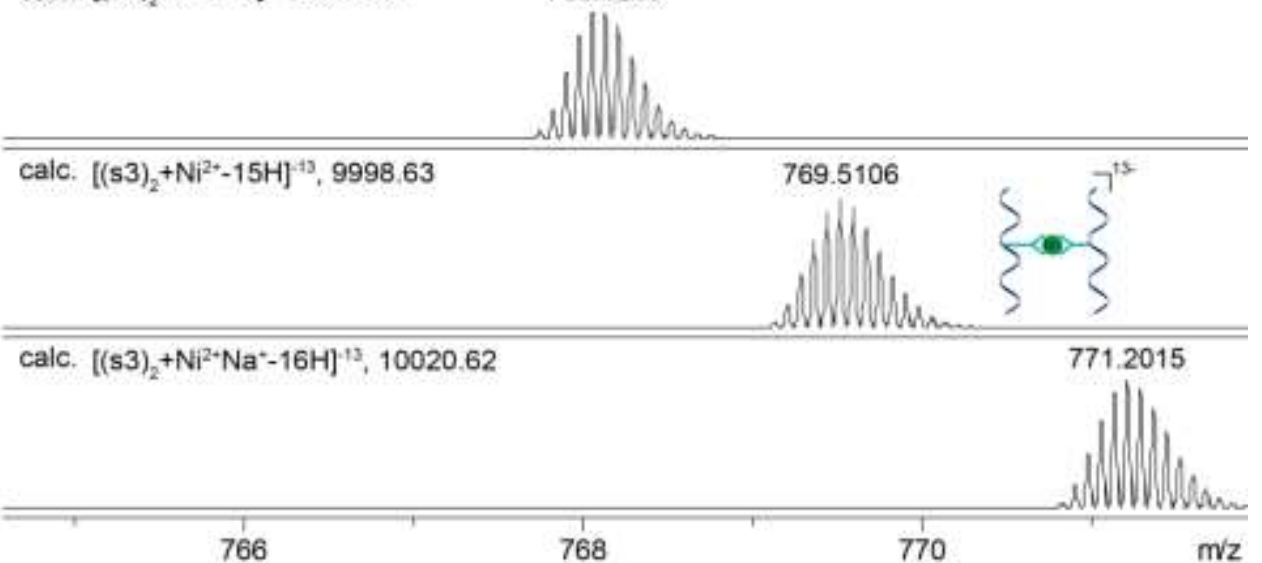

b) measured $m / z=-11$

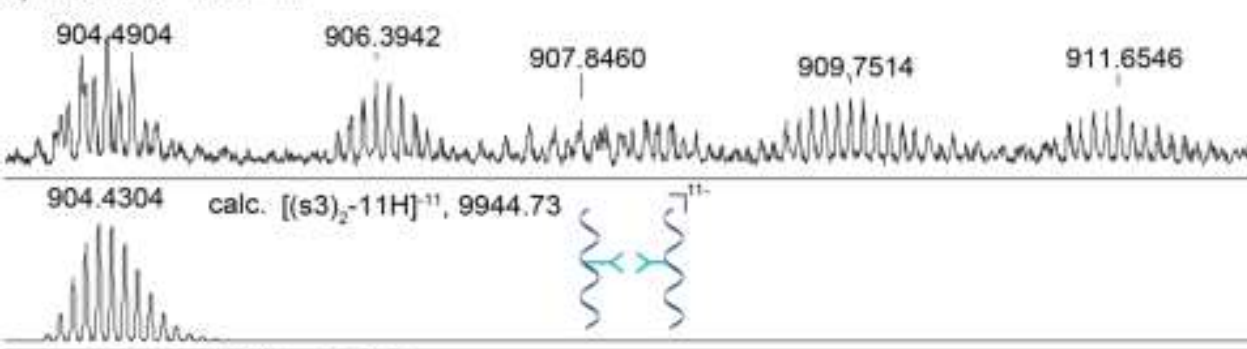

calc. $\left[(\mathrm{s} 3)_{2} \cdot \mathrm{Na}^{*}-12 \mathrm{H}\right]^{-11}, 9966.71$

906.4288

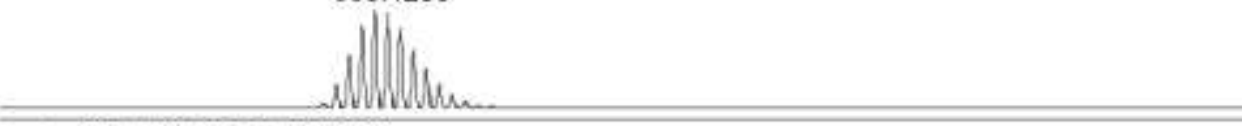

calc. $\left[(\mathrm{s} 3)_{2}, \mathrm{~K}^{*}-12 \mathrm{H}\right]^{-11}, 9982.69 \quad 907.7898$
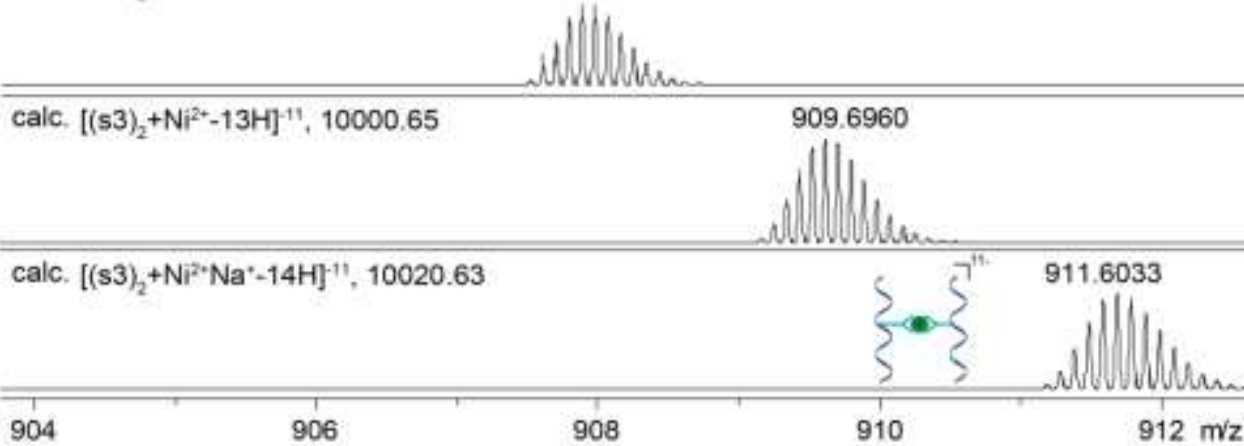

Figure 78. ESI-HRMS of $20 \mu \mathrm{M}$ ss-DNA s3 adducts with $\mathrm{Ni}(\mathrm{II}), \mathrm{H}_{2} \mathrm{O} / \mathrm{MeOH}(50: 50)$. a) Spectrum of the dimeric unmetallated and metallated adducts of the DNA intercross linked $\mathrm{m} / \mathrm{z}=-13$, 
b) Stacking of the spectrum of the dimeric adducts and calculated respective isotopic pattern for $m / z=-11$.

\subsubsection{Polyacrylamide gel electrophoresis, PAGE}

Nondenaturing $8 \%$ polyacrylamide gels conditioned with tris-borate magnesium (TBM) buffer (pH 8.3 and $\mathrm{Mg}(\mathrm{II}) 12 \mathrm{mM}$ ) were established as best conditions for running samples (500 $\mathrm{ng}$ ) of systems based on the modified single strand $\mathbf{s 3}$. The single strand $\mathbf{s 3}$, as can be observed in the Figure 79, contains a secondary structure that was concluded from preliminary gels where a line of the gel containing ss-DNA s3 indicated two spots. To make sure of it, both spots were visualized by UV shadow (due to the artificial nucleotide the DNA contains a strong emission of blue light when irradiated with UV lamp at $234 \mathrm{~nm}$ ) and cutted off. The DNA presented in both pieces of the gel were extracted (see on the experimental part for details) and loaded into a new gel. If the lines present spots running with different mobilities and do not indicate the formation of a both spots (at the same line) this would mean that they correspond to different oligonucleotide sequences (the previous sample loaded was not pure). But both lines containing the separated materials showed again the formation of two spots, which means a single sample of DNA which presents a secondary structure. This conclusion is consistent with the data obtained by ESI-MS, which indicactes the dimeric formation of unmetallated adduct made by two $\mathbf{3} 3$ strands through unspecific interactions.

The spots correspondent to the single strand $\mathbf{s} 3$ have different mobility. Based on the DNA ladder used, the first spot has a height between 10bp and 20bp of the DNA ladder. The second spot has a height similar to $20 \mathrm{bp}$. It is important to notice that the DNA ladder used is based on $d s$-DNA fragments and a direct comparison should not be done because ss-DNA and ds-DNA fragments containing the same total of nucleotides run with different mobility on the gel. Nevertheless, the 15 mer $\mathbf{s} 3$ has for the first spot a similar height to $15 \mathrm{bp}$ in the DNA ladder. The spot correspondent to the secondary structure though, did not presented a height correspondent to $30 \mathrm{bp}$ of the marker, but a similar height to $20 \mathrm{bp}$.

Under conditions of preparation of the loading sample for PAGE electrophoresis, the formation of secondary structure of the single strand $\mathbf{s} 3$ prevails upon formation of the duplex s3.s2* even under equivalent amount of $\mathbf{s} \mathbf{2}$ followed by proper cooling of the sample to allow hybridization of the duplex system. The reason is related to the small volume of the sample $(15 \mu \mathrm{l})$ and the ionic strength (12 $\left.\mathrm{mM} \mathrm{Mg}(\mathrm{OAc})_{2}\right)$ for gel, which favors the secondary structure of $\mathbf{s} \mathbf{3}$ rather than the hybridization with $\mathbf{s 2 *}$. 
The prevalence of secondary structures dependent on the ionic strength over the duplex formation can be observed in the literature ${ }^{[4]}$ and in cases of intramolecular equilibrium (hairpin) as observed in part $\mathbf{2}$ for the strand $\mathbf{s 1},{ }^{[37]}$ which actually has the same nucleotide sequence of strand $\mathbf{s} \mathbf{3}$ (with the difference that $\mathbf{s} \mathbf{3}$ contains an artificial nucleotide with the trans-chelating motif pointing outside of the strand and not incorporated internally in the sequence). As was observed by CD spectroscopy (Figure 71, section 2.3.3.3) the strand $\mathbf{s 3}$ also presents, such as strand s1, a band (range $366-408 \mathrm{~nm}$ ) attributed to unspecific interactions related to some nucleobases of the sequence with the artificial ligand incorporated, but in this case the formation of hairpin was not observed by control measurements of thermal analysis. Although, such interactions produce an unspecif secondary structure, possibly related to the modification and dependent of ionic strength, that is favored due to the low volume of the sample for loading conditions in the gel.

Figure 79a shows that the gel after staining (SYBR ${ }^{\circledR}$ Gold nucleic acid gel stain) has a faint upper spot around 30bp, that was assigned to the formation of a small amount of duplex s3.s2* and the predominant amount of sample that remained as sepparated single strands $\mathbf{s} 3$ and $\mathbf{s} \mathbf{2}^{*}$ (the latter is probably overlapped with the first spot of $\mathbf{s 3}$ ). Notice that the artificial sequence $\mathbf{s 3}$ has a stronger color than $\mathbf{s 2 *}$. This is due to the fluorescence of the DNA sequence, been able to be visualized before staining (Figure 79b). After staining, the spot for $\mathbf{s 2 *}$ has the fluorescence only from the SYBR Gold stain, while the strand $\mathbf{s} 3$ has a combination of fluorescences (from its artificial nucleotide and from the stain used) which can be noticed by a stronger color (bright yellow under brown filter visualization (Figure 79a) or as a darker spot after conversion of the picture to black and white (Figure 79c)).

Attempts by PAGE to detect the interduplex cross-link upon addition of $\mathrm{M}(\mathrm{II})$ were perfomed and, as explained above, the hybridization of DNA duplex was not succeed and the efforts were focused to obtain a possible inter cross-link of a system based on the single strand $\mathbf{s 3}$. The Figure 79 below shows in line 5 a splitting of both spots for the single strand s3. Such splitting is attributed to the coordination with $\mathrm{Cu}(\mathrm{II})$ for both spots, which indicates for the first spot a monomer complex and for the second spot (the secondary structure of $\mathbf{s} 3$ ) a possible dimer complex, that means the inter cross-link between two single strands $\mathbf{s} 3$. This data is also consistent to the ESI-MS obtained. 


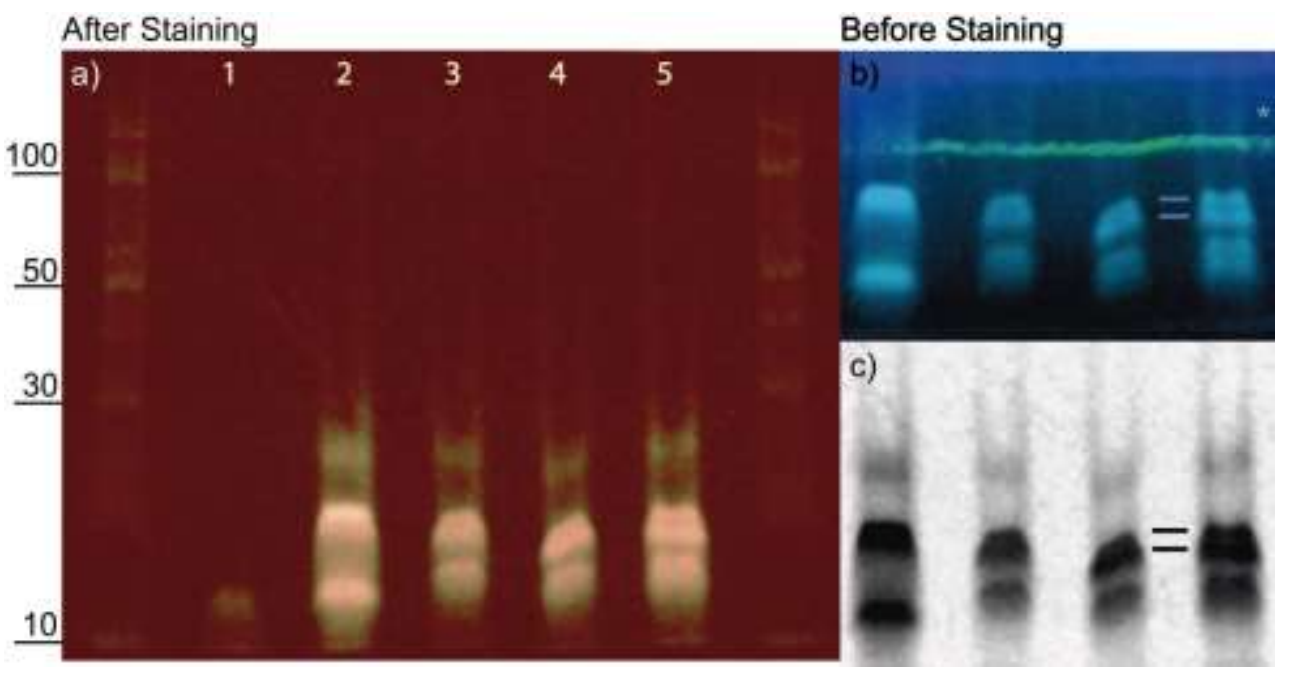

Figure 79. Native TBM gel $8 \%$ for 500 ng s3.s2* with $M(I I)$. a) Visualization of the full gel after staining with use of sepia filter, b) Close-up picture for lanes 2 to 5 before staining, indicating blue fluorescence color from the single strand s3, c) Closed-up picture for lanes 2 to 5 after staining and converted to black and white. Lane 1: ss-DNA s2*, lane 2: ss-DNA s2*+ss-DNA s3, lane 3: ss-DNA s2*+ ss-DNA s3 + 0.5 eq. Cu(II), lane 4: ss-DNA s2*+ss-DNA s3 + 0.5 eq. Ni(II), lane 5: ss-DNA s2*+ss-DNA s3 + 1.0 eq.Cu(II). The lanes before lane 1 and after lane 5 were loaded with samples containing DNA ladder (150-10 bp).

A longer incubation (48 h) of the loading sample for strand s3 (details at the experimental part) was found out as a way to obtain a full conversion of the single strand $\mathbf{s} \mathbf{3}$ to its secondary structure. The gels containg s3 as total secondary structure indicates a slight change on the mobility; the spot runs a bit faster in comparison to the DNA ladder, been a bit further to the mark for 20bp. The absence of the spot correspondent to the unwind $\mathbf{s} 3$ strand gives a clear visualization for the unhybridized single strand s2* (Figure 80, lower spot from lanes 4 to 7 ).

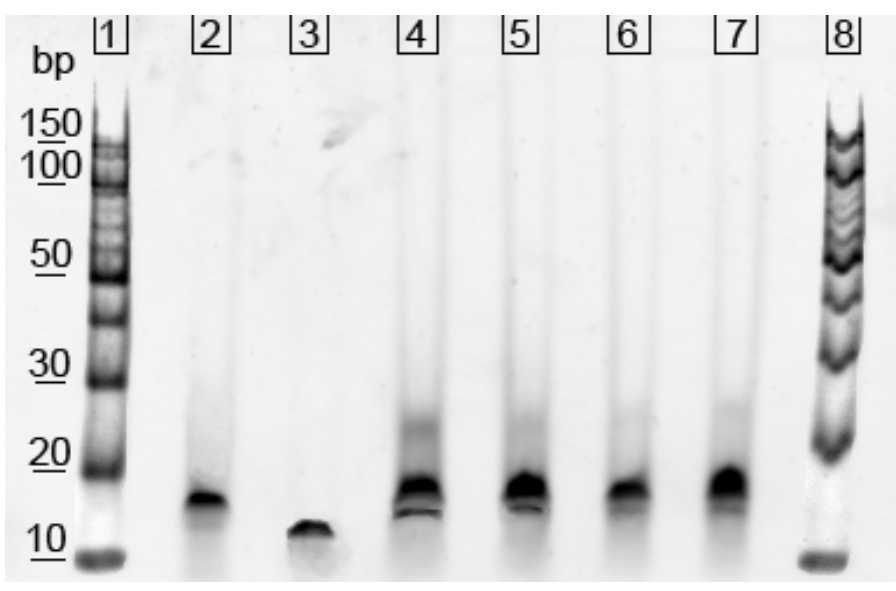

Figure 80. Native 8\% TBM gel for 500 ng s3.s2* with $M(I I)$. Lane 1 and 8: DNA ladder 15010 bp. Lane 2: ss-DNA s3, lane 3: ss-DNA s2*, lane 4: ss-DNA s2*+ss-DNA s3, lane 5: ss-DNA 
s2*+ ss-DNA s3 + 0.5 eq. Cu (II), lane 6: ss-DNA s2*+ss-DNA s3 + 0.5 eq.Pd(II), lane 6: ss-DNA s2*+ss-DNA s3 + 0.5 eq.Ni(II).

In Figure 80 it is possible to see also a slight formation of the duplex s3.s2* and the lanes 5 and 7 indicated a broader spot, a possible metal coordination with $\mathrm{Cu}(\mathrm{II})$ and $\mathrm{Ni}(\mathrm{II})$, respectively. All attemps of coordination with $\mathrm{Pd}(\mathrm{II})$ were not succeed. A previous work by Wagenknecht et al. presented a similar attempt with artificial nucleotide based on terpy group. In this work, it is possible to see similar evidences for metal complexation by spectroscopic methods while the gel for the single modified system presented several spots with different mobilities (for complexations with $\mathrm{Fe}(\mathrm{II})$ and $\mathrm{Ni}(\mathrm{II}))$ that indicates the existence of metallated DNA assemblies larger than dimers. ${ }^{[58]}$

The next gel (Figure 81) indicates a better and clear view of the dimer complex for $\mathrm{Cu}$ (II) (lane 4) with two s3 strands, as an upper spot to the one assigned as the secondary structure of s3. The lane 6 presents a broader spot indicating a possible monomer with $\mathrm{Cu}(I I)$, although did not present a clear resolution as the result obtained in the lane 4 . The formation of inter cross-link between two $\mathbf{s} 3$ strands was not possible under conditions that favors only the formation of its secondary structure. Due to that, the inclusion of complementary strand $\mathbf{s} \mathbf{2}^{*}$ was leading to misinterpretations, causing difficulties to assign the appearance of new spots as a possible formation of metallated $\mathbf{s 3}$ (first or secondary structure) or to unhybridized $\mathbf{s 2}$. Since the duplex is not been formed, the addition of $\mathbf{s} \mathbf{2}^{*}$ was discarded to avoid misinterpretations.

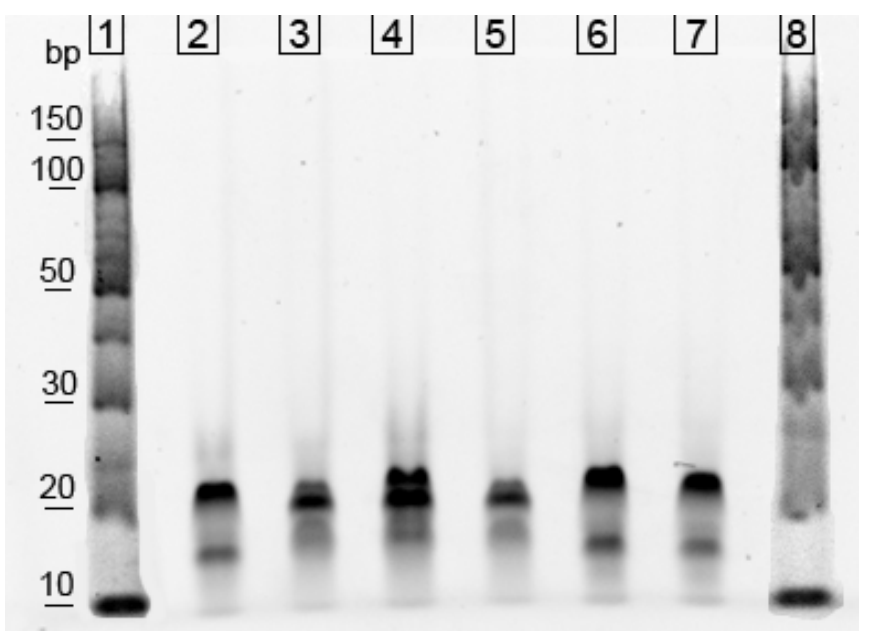

Figure 81. Native 8\% TBM gel for 500 ng s3 with $\mathrm{M}(\mathrm{II})$. Lane 1 and 8: DNA ladder 150-10bp. Line 2: ss-DNA s3, line 3: ss-DNA s3 + 0.5eq. Pd(II), lane 4: ss-DNA s3 + 0.5eq.Cu(II), lane 5: ssDNA s3 + 0.5 eq. $\mathrm{Ni}($ (II), lane 6: ss-DNA s3 + 1.0 eq. $\mathrm{Cu}(\mathrm{II})$, lane 6: ss-DNA s3 + 1.0 eq. $\mathrm{Ni}(\mathrm{II})$. 


\subsubsection{Results for the doubly modified system, s4.s5}

It is important to clarify some procedures concerning the preparation of the sample for metal complexation, which does not occur at room temperature nor in a brief time, due to kinetic limitations and weak binding constant. It is known for the ligand L2 presented in part $\mathbf{1}$ that the complexation with $\mathrm{Pd}(\mathrm{II})$ takes $4 \mathrm{~h}$ upon heating at $75^{\circ} \mathrm{C} .{ }^{[80]}$ In this part 3 , after every addition of metal to the DNA sample, the reaction was left heating for $12 \mathrm{~h}$ at $75^{\circ} \mathrm{C}$. Based on the melting point obtained in the section $\mathbf{2 . 3 . 4 . 2}$, it is known that $75^{\circ} \mathrm{C}$ is a temperature higher than the Tm for the duplex s4.s5 and because of it, it is necessary a slow cooling of the sample to ensure that the duplex is properly reanneled. Another possibility was also tried for the preparation of the sample, which resumed in heating the sample in temperature below the melting point $\left(40^{\circ} \mathrm{C}\right)$ for longer period (around one week) to obtain the full complexation in a process followed by the UV/Vis spectrum and the thermal analysis (sections 2.3.4.1 and 2.3.4.2 respectively). The final result indicated be the same for both alternatives (Figure 82). Although, if the samples after the metal coordination are submitted to the standard cooling adopted for hybridization (see in the experimental part) the result showed not be efficient to reproduce UV/Vis spectra under a constant absorption at $260 \mathrm{~nm}$, which means that it was happening a variation in the DNA concentration. Data obtained by thermal analysis demonstrated be unstable such as the results for PAGE electrophoresis. The specific cases will be discussed in the respective sections.

a)

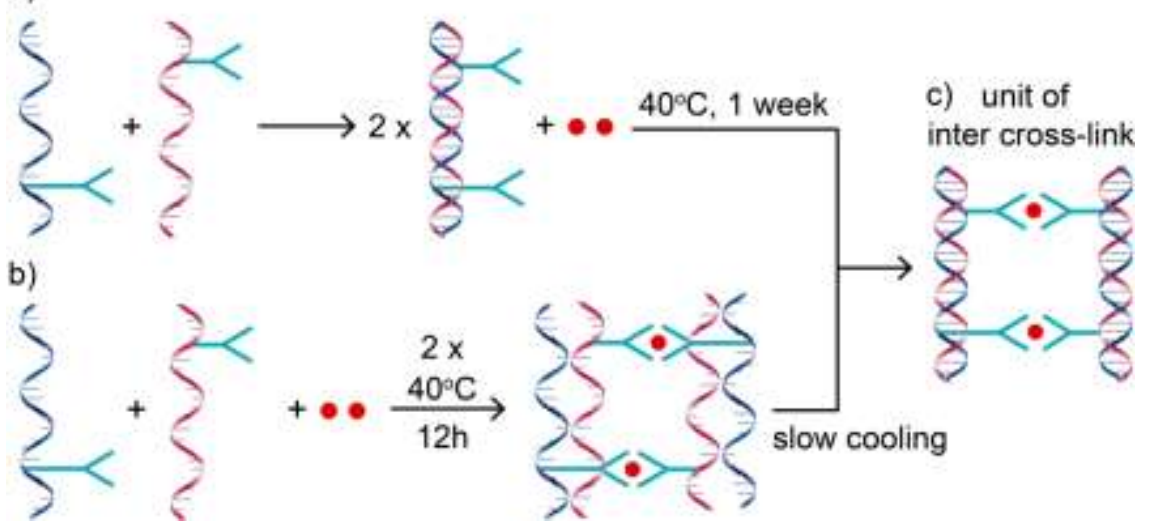

Figure 82. Scheme for the formation of the unit (c) of inter cross-link between two duplexes s4.s5 a) hybridization of the duplex s4.s5, followed by the $M(I I)$ complexation, b) metal complexation of the melted system followed by hybridization of $\mathbf{s 4 . s 5}$ under slow cooling producing the unit given in c).

Attemps to clarify such effect were based on the hypothesis of a polymerization of the DNA duplexes after the metal coordination. There is a possibility of the ss-DNA strands, s4 and s5, 
once melted, to rotate after metal coordination because of the alkyne linker that links the trans-chelating motif (Figure 83). The cooling parameter adopted for the reannealing process indicated be too fast to allow the coordinated single strands to hybridize properly and produce units of inter cross-links between two duplexes of s4.s5 (unit showed in Figure 82c).

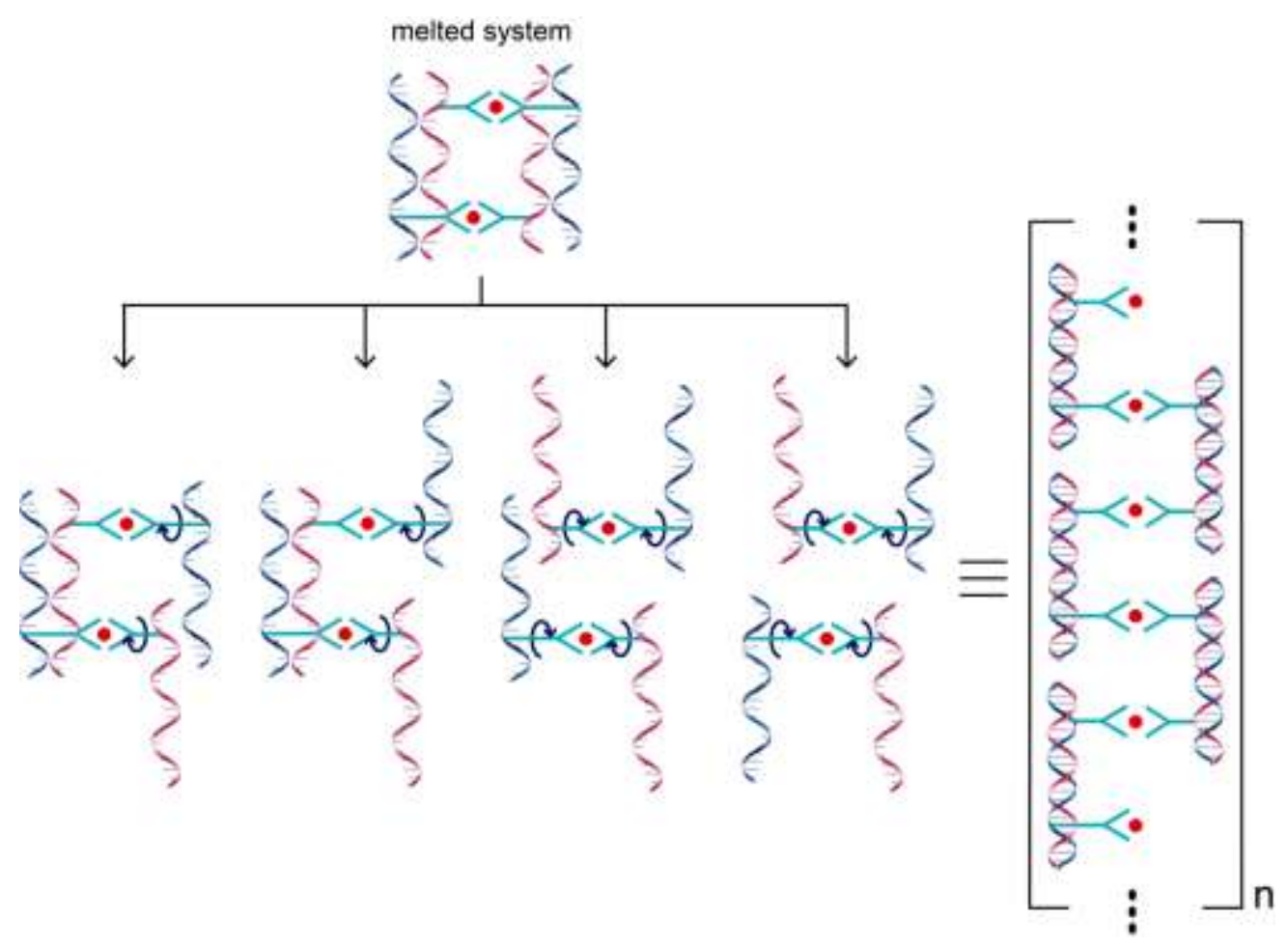

Figure 83. Scheme of possible spinning of the unhybridized system and final polymer product as a result of a fast cooling hybridization.

The system, coordinated but melted, allows the spinning of the single strands which can then polymerize during the process of hybridization giving a meta-stable product with formation of the duplexes between different units of complementary counterstrands (Figure 83). A similar issue was previously faced by Sleiman group in their work concerning DNA cyclic structures connected through ruthenium complexes incorporated into the DNA. ${ }^{[46]}$ The problem was sucessfully overcome under a slower cooling process allowing proper organized hybridization. The spectroscopic results showed in the next sections for metal coordinated samples of $\mathbf{s 4 . 5 5}$ were based on the same appproach concluded by Sleiman group, where the samples were submitted to a very slow cooling process (samples heated at $75^{\circ} \mathrm{C}$ for 20 minutes followed by a decrease of $1^{\circ} \mathrm{C}$ every 5 minutes until $4^{\circ} \mathrm{C}$ and later reaches the room temperature naturally) to induce a proper annealing and be able to obtain the desired units of interduplex cross-link instead of the polymerized product. The respective results that support such conclusions were discussed in the next sections for UV/Vis spectroscopy (2.3.4.1), thermal analysis (2.3.4.2) and gel electrophoresis. 


\subsubsection{UV/Vis spectroscopy}

The preliminary spectroscopic results for DNA duplex $\mathbf{5 4 . 5 5}$ with addition of $\mathrm{M}(\mathrm{II})$ demonstrated, through UV/Vis and thermal analysis, better evidences of the metal complexation with CHES buffer than similar assay in cacodylate buffer. The same sample of the duplex s4.s5 was used for all the spectroscopic measurements presented in the following sections, such as the metal additions. The conditions adopted were $4 \mu \mathrm{M} d s-\mathrm{DNA}, 150 \mathrm{mM}$ $\mathrm{NaCl}$ or $\mathrm{NaClO}_{4}, 50 \mathrm{mM}$ CHES buffer (pH 9.0). The UV/Vis measurements of the duplex s4.s5 presented the maximum of absorption at $260 \mathrm{~nm}$, as expected for DNA samples, and an additional band in the range of $300-420 \mathrm{~nm}$ related to the incorporated artificial nucleotide. Such profile is similar and consistent to the system s3.s2* (before and after addition of $\mathrm{M}(\mathrm{II})$ ) already discussed, but here it is expected that the UV/Vis spectra present a greater and clear view of the metal coordination in this region, due to the fact that the system $\mathbf{s 4 . s 5}$ is carrying two trans-chelating nucleotides per duplex. Additions of $\mathrm{Pd}(\mathrm{II})$ into the duplex $\mathbf{5 4 . 5 5}$ indicated a slight increase in the $A_{260}$, such fact was also observed for the systems s3.s2* and s1.s2 (part 2) and it is related to the metal addition into the sample. It was noticed a decrease of the absorption (Figure 84) between $300 \mathrm{~nm}$ (with maximum at $364 \mathrm{~nm}$ ) until the isosbestic point at $395 \mathrm{~nm}$ with a slight increase of the intensity after it until around $450 \mathrm{~nm}$, fact that is also similar to the spectra for $\mathbf{s 3 . s 2 *}$ indicating metal complexation.

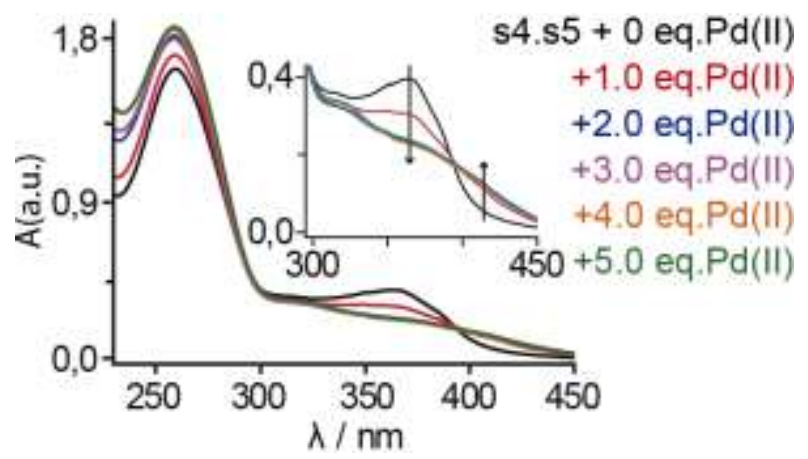

Figure 84. UV/Vis spectra for $4 \mu \mathrm{M}$ s4.s5, $150 \mathrm{mM} \mathrm{NaClO}_{4}, 50 \mathrm{mM}$ CHES buffer $+\mathrm{n}$ eq. of $\mathrm{Pd}(\mathrm{II})$.

For additions of $\mathrm{Pd}(\mathrm{II})$ after $2.0 \mathrm{eq}$. it was noticed the formation of a shoulder (at the range $305-333 \mathrm{~nm}$ ) with a slight increasing of the absorption in this area becoming more pronounced until stay constant upon the addition of 5 eq. of $\mathrm{Pd}(\mathrm{II})$. Despite the appearance of such shoulder, the position of the isosbestic point remained at $395 \mathrm{~nm}$ and the increase of the absorption after it was constant, indicating that there is not other component been formed upon higher additions of metal, thus such changes were simply related to a complete complexation of the system. 
The entire set of the administrated additions of $\mathrm{Pd}(\mathrm{II})$ in the sample can be seen in the Figure 85 , indicating that additions of 0.25 and 0.50 eq. of $\mathrm{Pd}(\mathrm{II})$ did not cause any change in the absorption nor in the profile of the band for the region attributed to the ligand, probably because of such amounts were not enough to promote the metal coordination. The first drop of the absorbance such as the appearance of the isosbestic point started upon addition of 0.75 eq. of $\operatorname{Pd}(\mathrm{II})$, becoming more pronounced until the addition of 1.50 eq. of $\mathrm{Pd}(\mathrm{II})$. The drop of the absorbance upon addition of $\mathrm{M}(\mathrm{II})$ at the point of the complex formation occurs in the characteristic abrupt profile already shown in the last DNA systems when using $\mathrm{NaClO}_{4}$ as electrolyte; while additions of $\mathrm{Cu}(\mathrm{II})$ in the presence of $\mathrm{NaCl}$ occurs more degressively.

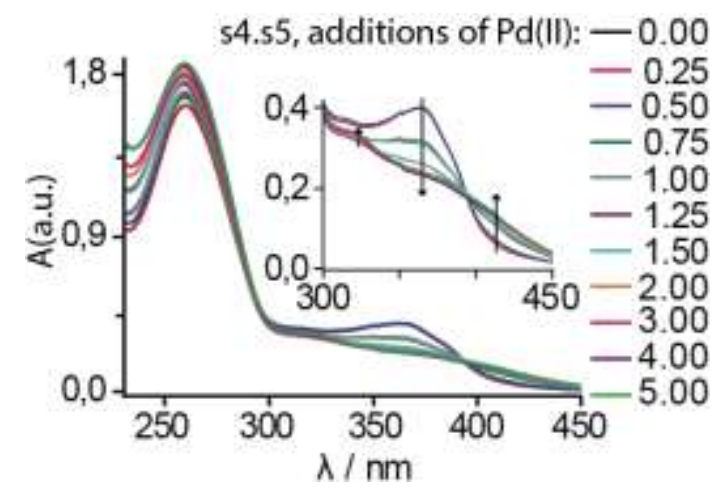

Figure 85. UV/Vis spectra for $4 \mu \mathrm{M}$ s4.s5, $150 \mathrm{mM} \mathrm{NaClO}_{4}, 50 \mathrm{mM}$ CHES buffer $+\mathrm{n}$ eq. of $\mathrm{Pd}(\mathrm{II})$.

The assay was repeated for additions of $\mathrm{Cu}(\mathrm{II})$ in $\mathrm{NaCl}$ as electrolyte instead of $\mathrm{NaClO}_{4}$ (Figure 86). The slow cooling procedure adopted to avoid the polymerization indicated not be fully efficient for complexation with copper(II). This could be concluded due to lower values for $A_{260}$. The lower values of $A_{260}$ indicates lower concentrations, which are probably related to a partial precipitation of the polymerized sample. The ratio of the polymerization is random, there is no quantitative parameter related to the addition of metal and the amount of sample that is polymerized. The respective thermal analysis of the sample for addition of $\mathrm{Cu}(\mathrm{II})$ indicated unreproducicle cycles (at $0.5^{\circ} \mathrm{C} / \mathrm{min}$ ) where each cycle was followed by hypochromism effect, another evidence of decrease of the absorption due to the precipitation. In the case of hyperchromism effect by thermal analysis and increase of the absorbance by UV/Vis, according to literature, it is attributed to formation of aggregates, which become suspended in the solution and cause light scattering. ${ }^{28]}$

It was only possible to notice that the variation of the concentration started for additions up to 0.50 eq. of $\mathrm{Cu}(\mathrm{II})$, amount also related to the formation of the isosbestic point at $395 \mathrm{~nm}$. In other words, once the complexation starts, the concentration indicates variations probably 
related to a partial polymerization of the system. The reason of it might be attributed to a stronger binding with $\mathrm{Cu}(\mathrm{II})$ than with $\mathrm{Pd}(\mathrm{II})$, where the polymer is the meta-stable state, the kinetic product while the unit of the inter cross-linked complex is the thermodynamic product, a conversion that was only fully possible for complexation with $\mathrm{Pd}(\mathrm{II})$ (when allowed the slow hybridization). Analysis of the decreasing of the absorption in the ligand area for complexation with $\mathrm{Cu}(\mathrm{II})$ such as the slight increasing after the isosbestic point could not be done precisely because of the variation of the concentration.
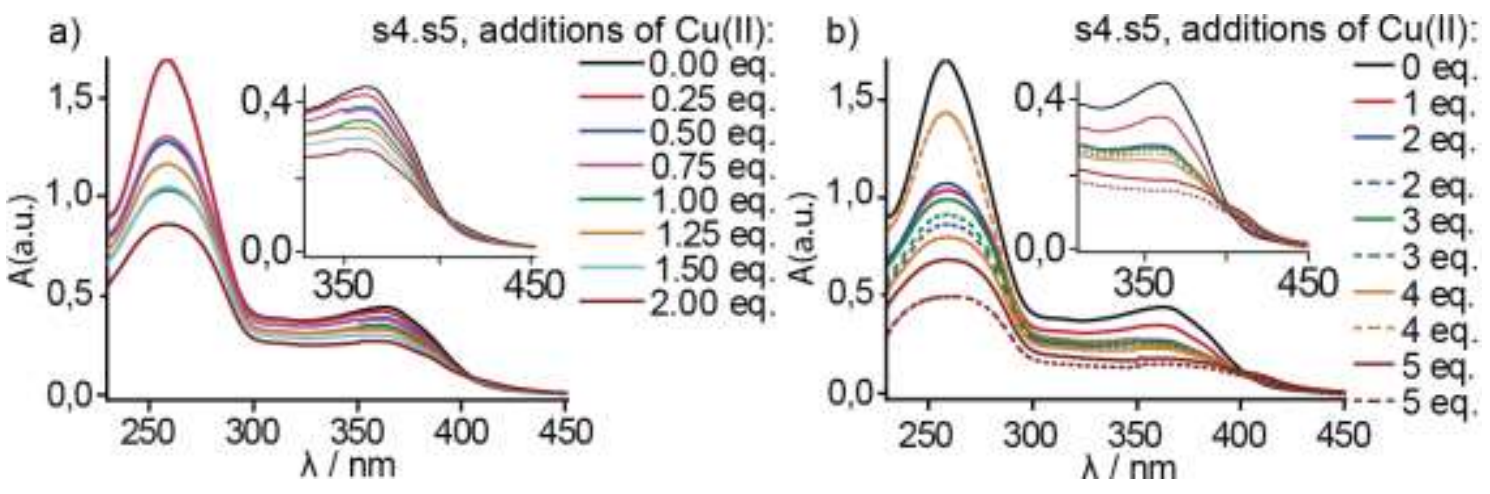

Figure 86. a) UV/Vis spectra for different additions of $\mathrm{Cu}(\mathrm{II})$ amounts into the system s4.s5 until 2 eq. of $\mathrm{Cu}(\mathrm{II})$. b) UV/Vis spectra showing the difference in the sample concentration for the sample before (solid line) and after (dashed line) submitted to slow cooling for annealing process to avoid polymerization.

Notice that in Figure 86a the different values for absorption at $260 \mathrm{~nm}$, which should be constant. In Figure $86 \mathrm{~b}$ the oscilation of the concentration at the same amount of $\mathrm{Cu}(\mathrm{II})$ shows the different amount of product formed as unit and as polymer of interduplex cross-link.

\subsubsection{Thermal analysis}

The melting curve analysis of the absorbance in function of the temperature were performed to obtain information concerning the duplex stability of the modified DNA duplex s4.s5 before and after metal coordination. First of all, the comparison of the doubly modified system with the unmodified duplex $\mathbf{s} 4^{*} . \mathbf{s} 5^{*}$ (containing a dT instead of $X$ for single strand $s 4^{*}$ and $d A$ instead of $\mathrm{X}$ for $\mathbf{s} 5^{*}$ ) indicated a less stable system. The assay performed with $\mathrm{NaClO}_{4}$ presents a lower hysteresis than using $\mathrm{NaCl}$ as electrolyte. It is interesting to see how the change on the electrolyte can cause a such significant change in the system. 

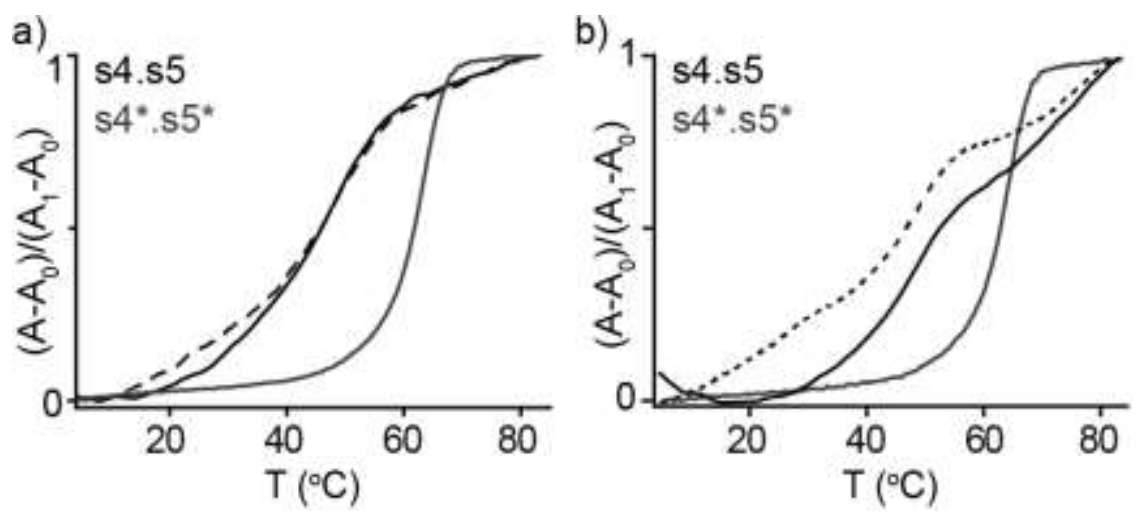

Figure 87. Thermal analysis for $4 \mu \mathrm{M}$ s4.s5 and s4*.s5*, $50 \mathrm{mM}$ CHES buffer, a) $150 \mathrm{mM}$ $\mathrm{NaClO}_{4}$, b) $150 \mathrm{mM} \mathrm{NaCl}$. Values in parenthesis are for the reannealing process, temperature rate of $0.1^{\circ} \mathrm{C} / \mathrm{min}$.

Similar melting curve profile was observed (Figure 87) for unmodified duplex s4*.s5* in both types of electrolytes, but great difference when the conditions were submitted to the modified duplex s4.s5, with a slight hysteresis for assay performed with $\mathrm{NaClO}_{4}$ (Figure 87a) presenting $\Delta \mathrm{Tm}=-16.5(14.5)^{\circ} \mathrm{C}$ and very distinct kinetic paths for melting and reannealing processes in $\mathrm{NaCl}$ (Figure $87 \mathrm{~b}$ ) with $\Delta \mathrm{Tm}=16.4(15.4)^{\circ} \mathrm{C}$. It is not clear for the assay in $\mathrm{NaCl}$ if the melting profile indicates a very broad transition or if is only seen the first melting point with a second one out of the temperature range. Independent of the salinic source used, the assay upon addition of $\mathrm{Cu}(\mathrm{II})$ indicated a too complex melting transition with the profile of the curves totally altered from the sigmoidal shape in case of additions Cu(II) (appendix Figure 99 ). Such difficulties are probably related to the fast formation of intermediates or polymerization of the system, even under the melting experiments been performed under a rate of $0.1^{\circ} \mathrm{C} / \mathrm{min}$, that was necessary to provide reproducible melting cycles. The discussion will be focused in systems with additon of $\mathrm{Pd}(\mathrm{II})$ in $\mathrm{NaClO}_{4}$, whithin were able to present reproducible cycles under constant duplex concentration and reasonable melting curve profiles (not deviating from the sigmoidal shape) that allowed an interpretation.

The system s4.s5 in $\mathrm{NaClO}_{4}$ indicated a slight hysteresis between the melting (solid black line, Figure 87a) and reannealing processes (dashed black line 87a), which means distinct kinetic paths fot the melting and reannealing processes with a broader transition and a $\Delta \mathrm{Tm}=-16.5(-$ $14.5)^{\circ} \mathrm{C}$ (values in paranthesis for the reannealing process) in comparison to the duplex s4*.s5*

The addition of 1 eq. of $\operatorname{Pd}(\mathrm{II})$ to the system demonstrated a very slow kinetic process for the $\mathrm{Pd}(\mathrm{II})$ complexation, where every followed melting cycle gained a shift forward in the melting temperature (Figure $88 \mathrm{~b}$ and c). The melting curve kept been recorded in a total of 14 cycles, 
when the system finally indicated be reproducible (complete metal-complexation) at the $12^{\text {th }}$ cycle. The Figure $88 \mathrm{~b}$ ) and c) shows selected melting and reannealing cycles for the addition of 1 eq. of $\operatorname{Pd}(I I)$. The processes were separated to facilitate the visualization and the high hysteresis between both processes (melting and annealing) is evidence of different kinetic paths, such as values for the melting points obtained. In this case, the literature says that none of both values obtained represent the real value of the melting point (with one of the values been always overrated while the another is underrated). ${ }^{[60]}$ Because the reannealing process, in cases of complexes transitions, takes into account not only the zippering but also nucleation mechanism ${ }^{[4,66]}$, we decided to assign, for our analysis and comparisons for additions of metal performed here in this work, the values of melting point given by the reannealing process.
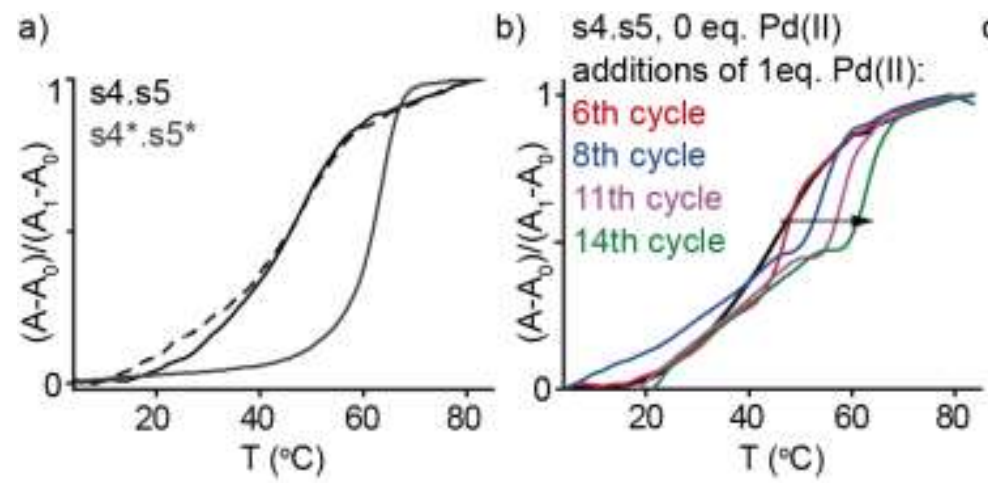

c) $s 4 . s 5,0$ eq. $P d(I I)$

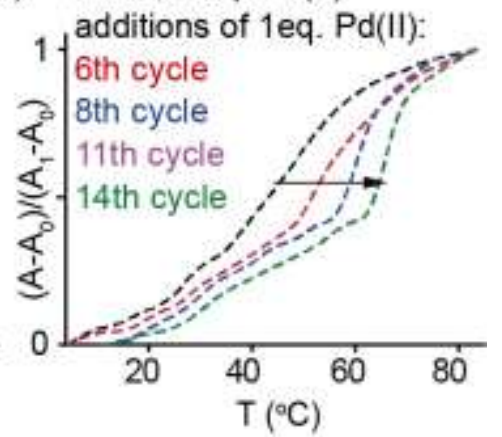

Figure 88. Thermal analysis for DNA duplexes a) $\mathbf{s 4 . s 5}$ and $\mathbf{s 4 * . s 5 * ( s o l i d ~ b l a c k ~ l i n e ~ f o r ~ m e l t i n g ~}$ process and dashed black line for renaturing process), b) melting processes for several cycles of s4.s5 upon addition of 1 eq. of $\mathrm{Pd}(\mathrm{II})$, c) reannealing process for several cycles of $\mathbf{s 4 . s 5}$ upon addition of 1 eq. of $\mathrm{Pd}(\mathrm{II})$.
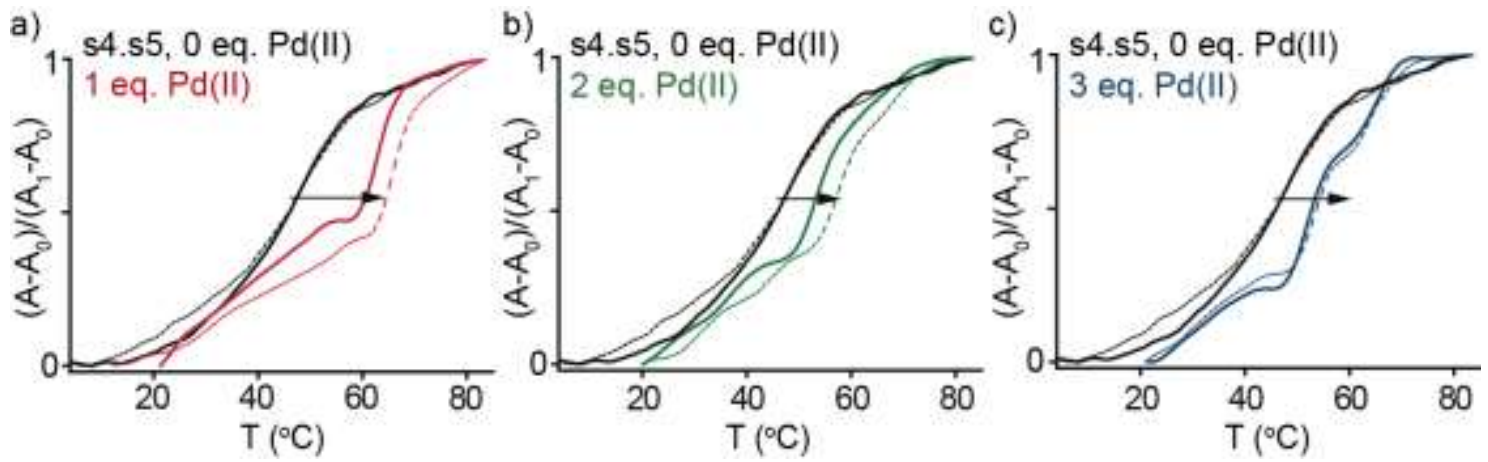

Figure 89. Thermal analysis for $4 \mu \mathrm{M}$ s4.s5, $150 \mathrm{mM} \mathrm{NaClO}_{4}, 50 \mathrm{mM}$ CHES buffer, upon additions of $\operatorname{Pd}(I I)$, a) 1 eq, b) 2 eq, c) 3 eq.

The final reproducible cycle for addition of 1 eq. of $\mathrm{Pd}(\mathrm{II})$ can be visualized in comparison to the unmetallated system in Figure 89a. The hysteresis became more pronounced and as mentioned, this is due to the fact that the paths for the melting and reannealing transitions 
after the metal coordination become more distinct. The change in the melting point observed for the system with addition of 1 eq. of $\mathrm{Pd}(\mathrm{II})$ provides a system more stable, as a possible product of the metal complexation, with $\Delta \mathrm{Tm}=+17.0^{\circ} \mathrm{C}$ (based on values for the reannealing process).

Further additions of $\mathrm{Pd}(\mathrm{II})$ indicated a system less stable for addition of 2 eq. of $\mathrm{Pd}(\mathrm{II})$ in comparison to 1 eq. of $\mathrm{Pd}(\mathrm{II})$ (Figure $89 \mathrm{~b})$ ), although still more stable than the system unmetallated $(\Delta \mathrm{Tm}=+8.0)$ where the hysteresis of the melting curve profile remained expressive. For the addition of 3 eq. of $\mathrm{Pd}(\mathrm{II})$ the melting transition presented a reduction in the hysteresis and a second melting point that indicates a transition of a cooperative system. The first melting occurs at $51.6^{\circ} \mathrm{C}$ and the second at $65.0^{\circ} \mathrm{C}$. An interpretation suggested it that for additions of $\mathrm{Pd}(\mathrm{II})$ lower than 3 equivalents the system does not present both artificial nucleotides at the same duplex coordinated to Pd(II). The unit formed from an inter cross-link between two duplexes $\mathbf{s 4 . 5 5}$ would be linked by a single metal coordination (which also can justify a higher hysteresis because the formation of an unit less rigid as product). Upon addition of 3 eq. of $\mathrm{Pd}(\mathrm{II})$ the unit formed is doubly metal coordinated, which indicates a rigid system with reduced hysteresis and two melting points.

Nevertheless, this is just a suggestion of a possible interpretation for the data showed. From the melting curve profile obtained the only points that can be affirmed with sure are the fact of the system after addition of $\operatorname{Pd}(I I)$ be more stable, with a slow kinetic for the metal complexation and the distinct kinetic paths for the melting and reannealing processes.

Table 7. Values of the melting points for $4 \mu \mathrm{M} d s-\mathrm{DNA}$, s4*.s5* and s4.s5, $150 \mathrm{mM} \mathrm{NaClO}$, 50 mM CHES buffer, upon addition of Pd(II). * in comparison to the unmodified duplex s4*.s5*

\begin{tabular}{c|c|c|c}
\hline DNA duplex & $\begin{array}{c}\text { Addition of } \\
\mathrm{n} \text { eq. of } \mathrm{Pd}(\mathrm{II})\end{array}$ & $\begin{array}{c}\mathrm{Tm}\left({ }^{\circ} \mathrm{C}\right) \text { ( value } \\
\text { for reannealing) }\end{array}$ & $\begin{array}{c}\Delta \mathrm{Tm}\left({ }^{\circ} \mathrm{C}\right) \text { of } \mathbf{s 4 . s 5} \text { for } \\
+\mathrm{n} \text { eq. of } \mathrm{Pd}(\mathrm{II})\end{array}$ \\
\hline $\mathbf{s 4 * . s 5 *}$ & 0 eq. & 63.5 & --- \\
$\mathbf{s 4 . s 5}$ & 0 eq. & $47.0(49.0)$ & $16.5(14.5)^{*}$ \\
s4.s5 & 1 eq. & $63.0(66.0)$ & $+16.0(+17.0)$ \\
s4.s5 & 2 eq. & $53.0(57.0)$ & $+6.0(+8.0)$ \\
s4.s5 & 3 eq. & $\mathrm{Tm}_{1} 51.6$ & $-1.4(---)$ \\
& & $\mathrm{Tm}_{2} 65.0$ & $---(---)$ \\
\hline
\end{tabular}




\subsubsection{Circular dichroism spectroscopy}

Circular dichroism spectra indicated expected Cotton effect attributed to B-DNA structure, with a negative band around $250 \mathrm{~nm}$ and a positive band at $280 \mathrm{~nm}$. Both single strands, s4 and $\mathbf{s 5}$ were measured separately $\left(\mathrm{NaClO}_{4}\right.$ and $\mathrm{NaCl}$, Figures $90 \mathrm{a}$ and $91 \mathrm{a}$, respectively) and did not indicate any secondary structure formed or chirality transfer onto the ligand. The formation of the duplex s4.s5 has an increase of the signal intensity due to the formation of the double helix and $\pi$-stacking interactions between the neighbouring bases. A slight deviation of the signal further $300 \mathrm{~nm}$ was closer to zero line after the addition of metal (effect observed for $\mathrm{Pd}(\mathrm{II})$ and $\mathrm{Cu}(\mathrm{II})$, Figures $90 \mathrm{~b}$ and $91 \mathrm{~b}$ respectively).

a)

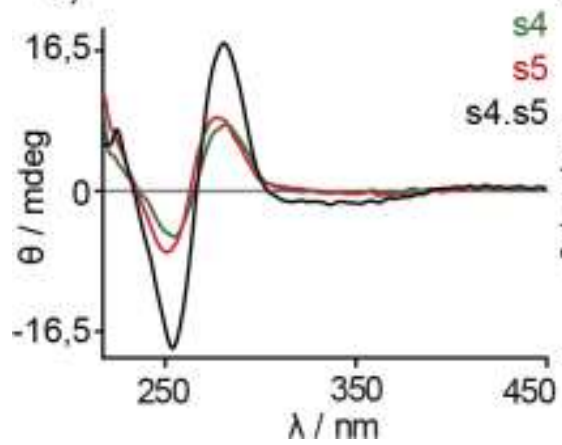

b)

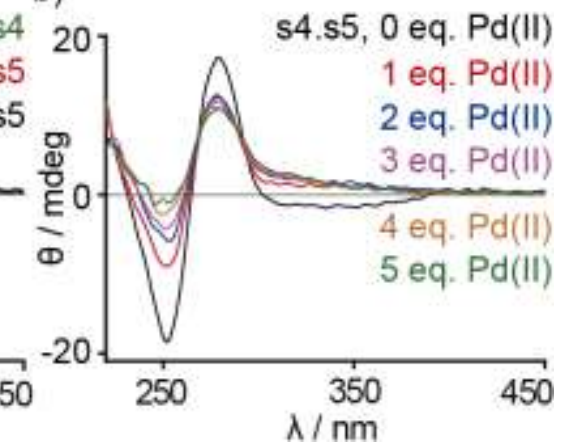

Figure 90. CD spectra for $4 \mu \mathrm{M}$ of a) s4, s5 and s4.s5, $150 \mathrm{mM} \mathrm{NaClO}{ }_{4}, 50 \mathrm{mM}$ CHES buffer and b) $C D$ spectra for $\$ 4.55+n$ eq. of $P d(I I)$.

Upon the addition of 5 eq. of $\mathrm{Pd}(\mathrm{II})$ (Figure 90a) the spectra of CD indicated be almost featureless in the negative band. This observation suggests an undeterminated change in the conformation, what can be an evidence of a mechanism where the metal-complexation by inter cross-link of the artificial nucleotides decrease the helicity around the neighbouring canonical base pairs of the artifical nucleotides compromised by the formation of the complex. Although, such effect was not observed in the assay upon addition of $\mathrm{Cu}(\mathrm{II})$ (Figure 91b).

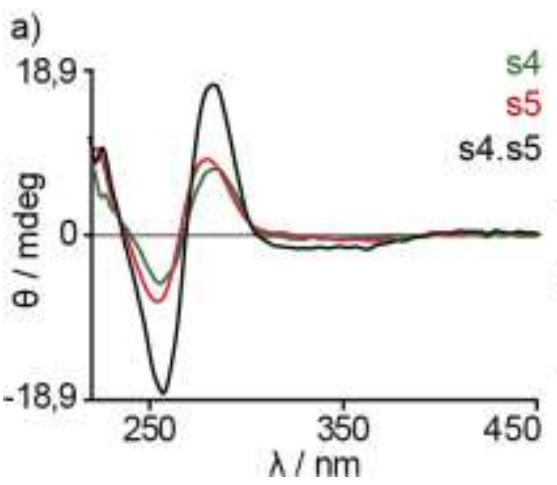

b)

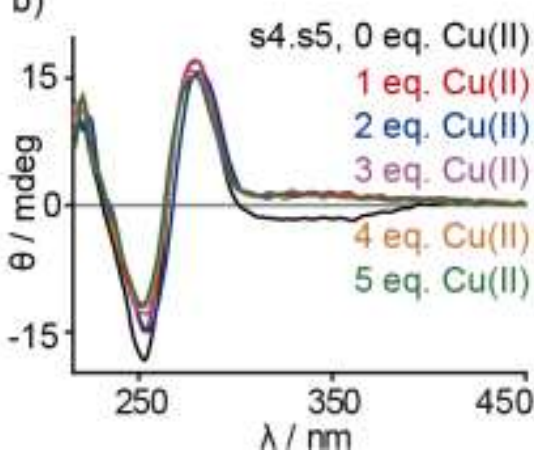

Figure 91. CD spectra for $4 \mu \mathrm{M}$ of a) s4, s5 and s4.s5, $150 \mathrm{mM} \mathrm{NaCl}, 50 \mathrm{mM} \mathrm{CHES}$ buffer and $\mathrm{b}$ )

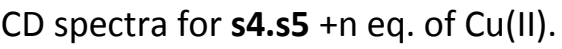




\subsubsection{Fluorescence Spectroscopy}

Emission spectra were collected with excitation at $360 \mathrm{~nm}$ and showed an emission range at $375-600 \mathrm{~nm}$. The metal coordination through the trans-chelating motif with $\mathrm{Pd}(\mathrm{II})$ or $\mathrm{Cu}(\mathrm{II})$ caused a quenching in the fluorescence intensity, attributed to the artificial nucleotide, as expected and indeed observed for both $M(I I)$ (Figure 92). The quenching of the fluorescence was greater effective for the addition of $\mathrm{Pd}(\mathrm{II})$ than $\mathrm{Cu}(\mathrm{II})$, a pattern that was noticed for all the other systems already investigated (s3.s2* and $\mathbf{s 1 . s 2}$ in part 2). A percentage of the quenching was also calculated based on the the literature according to the value of the maximum $(423 \mathrm{~nm})$ and presented in the following table.
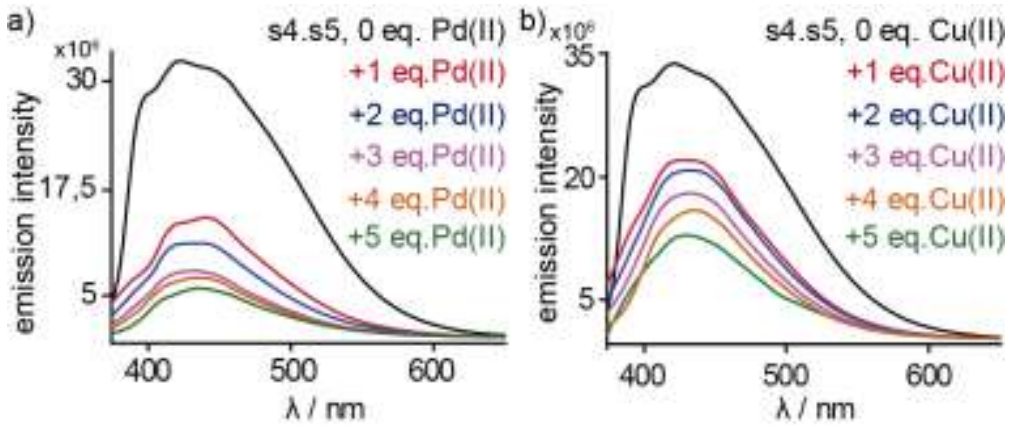

Figure 92. Emission spectra for $4 \mu \mathrm{M}$ s4.s5, $50 \mathrm{mM}$ CHES buffer, a) $150 \mathrm{mM} \mathrm{NaClO}{ }_{4}$ and b) $150 \mathrm{mM} \mathrm{NaCl}$ upon addition of $\mathrm{Pd}(\mathrm{II})$ and $\mathrm{Cu}(\mathrm{II})$ respectively.

Table 8. Percentage quenching of $\mathbf{s 4 . s 5}$ system upon addition of $M(I I)$.

\begin{tabular}{c|c|c}
\hline n eq. of $M(I I)$ & $\begin{array}{c}\text { \% of quenching } \\
\text { with } \mathrm{Pd}(\mathrm{II})\end{array}$ & $\begin{array}{c}\text { \% of quenching } \\
\text { with } \mathrm{Cu}(\mathrm{II})\end{array}$ \\
\hline 1eq & 58.7 & 35.5 \\
2eq & 66.5 & 39.4 \\
3eq & 76.0 & 42.3 \\
4eq & 79.4 & 55.7 \\
5eq & 83.7 & 63.0 \\
\hline
\end{tabular}

\subsubsection{Polyacrylamide gel electrophoresis, PAGE}

The doubly modified system $\mathbf{\$ 4 . 5 5}$ contains a single modification incorporated into each single strand and because of it and the volatilization conditions for ESI-MS, the duplex s4.s5 
denatures, not been possible to obtain any evidence of the DNA metal adduct formed by the inter cross-link between two duplexes. It was also not possible to perform similar FRET assay as the one made for the system $\mathbf{s} 3 . \mathbf{s} 2^{\mathrm{D}}$, because one of the single strands should also be carrying the FRET pair. A similar assay could be done with a single doubly modified strand, s6, but unfortunately this strand as already explained was not possible to be sucessfully purified. Based on the dificulties explained, the only more concrete evidence of inter cross-link between two $\mathbf{4 4 . 5 5}$ upon addition of M(II) was obtained by PAGE electrophoresis.

The gel preparation followed the same procedure for the system $\mathbf{s} 3+\mathrm{Cu}(\mathrm{II})$. In the system $\mathbf{s 4 . s 5}$ was possible to observe the hybridization of the double helix s4.s5, that based on UV/Vis and thermal analysis did not indicated a possible wrappring or interactions of the single strands to compete with the duplex formation under concentrated salty solutions. It is possible to see (lane 3, Figure 93) the full hybridization upon equimolar additions of $\mathbf{s} 4$ and $\mathbf{s 5}$ followed by proper incubation. The mobility of the spot seems a bit higher than the expected for $40 \mathrm{bp}$, probably due to the artificial incorporation of two trans-chelating nucleotides. Invitrogen 10bp DNA ladder was used and demonstrated not satisfactory resolution to establish a good comparison of mobility with the system s4.s5. Because of this reason, the system was performed again with another DNA ladder (O 'Range Ruler 10-150 bp by Thermo Fischer) as can be observed next.

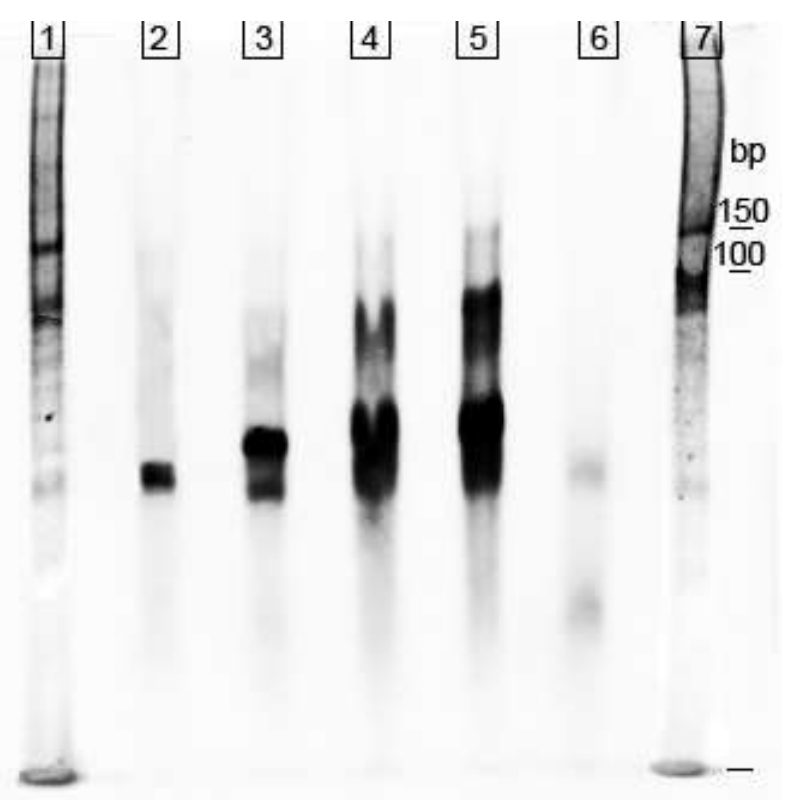

Figure 93. Native 8\% polyacrylamide gel in TBM buffer. Lanes 1 and 7: DNA ladder, lane 2:

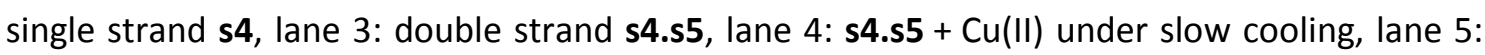
s4.s5 + Ni(II), lane 6: s4.s5 + Cu(II) under fast cooling. 
The interpretation of the gel (Figure 93) is the formation of a metallated DNA adduct upon addition of $\mathrm{Cu}(\mathrm{II})$ and $\mathrm{Ni}(\mathrm{II})$, as can be seen in the respective lanes (4 and 5) by the appearance of a higher spot. The comparison with the DNA ladder is subjective, not only due to the low resolution presented by the fragments, but also because a possible dimerization through inter cross-link between the duplexes would give a structure that has in fact similar number of basepairing, but would have a larger width for the structure formed. The unit of nanoconstruct obtained is shorter and bulker and possibly has different mobility from an elongated B-DNA duplex with $40 \mathrm{bp}$ and $80 \mathrm{bp}$ (to compare with an inter crossed-link dimer), as occur to the fragments of the ladder. The best would be have a known bulky system with similar number of base-pairs to use as a reference.

Although, it was concluded that the higher spot corresponds to a dimeric metallated structure because of procedures of different heating and cooling performed in the loading samples. The lanes 4 and 6 contain the same sample, which was made in double amount. After heating and incubation for metal complexation the sample was divided into two parts, where one half (lane 4) was submitted to a slow cooling process to avoid possible polymerizations (heated until $80^{\circ} \mathrm{C}$ and slowly cooled until $4^{\circ} \mathrm{C}$ at a rate of $1^{\circ} \mathrm{C} / \mathrm{min}$ ). The another half of the sample (lane 6) was allowed to cool down by the conventional method adopted (supporting information), which is a too fast cooling not providing conditions of the sample to hybridize properly, hence the polymeric form. The polymeric form is too big and large to penetrate inside of the porous of the gels (running gel 8\%, stacking gel 4\%). As can be observed in Figure 93 (lane 6) there are no trace of the metallated spot as occurs in lane 4 , with only traces of unmetallated single strands s4 and duplex s4.s5. The majority amount of the loaded sample probably kept in the surface of the running buffer as polymerized product.

Previous tries to the gel presented in Figure 94 yielded to several failures, where was not been understood, why the gels after be properly loaded and ran looked as empty (Figure 94a). This happened because the metallated DNA samples were not submitted to a proper slow cooling process for hybridization and probably only the product as polymer was obtained. Only faint traces of the samples could be visualized in random cases, where was possible to observe a strong spot in the surface of the loading gel $(4 \%)$, probably due to the formation of smaller polymer fragments. Different conditions were performed until be found the proper slow cooling procedure to avoid such polymerization. In Figure $94 \mathrm{~b}$ it is possible to see a gel of a sample submitted to a intermediate slow cooling, although still not enough to produce a sample to run proper through the gel. The samples were possibly fragments of higher molecular weight and lower mobility (also possible to see higher polymeric formation in the 
loading gel, as darker traces marked by arrows). The proper conditions of cooling that allow us to obtain the dimeric metallated-DNA constructs indicates remaining traces of polymerization, as can be observed in Figure 94c. This evidence is coherent to the UV/Vis and thermal analysis, which indicates for $\mathrm{Cu}(\mathrm{II})$ and $\mathrm{Ni}(\mathrm{II})$ a not complete conversion for the dimerized unit product even under slow cooling. The complete conversion for the dimer-unit product demonstrated be complete (based on UV/Vis and thermal analysis) only for complexes obtained with $\mathrm{Pd}(\mathrm{II})$, which unfortuantely were not possible to provide positive results for PAGE electrophoresis.

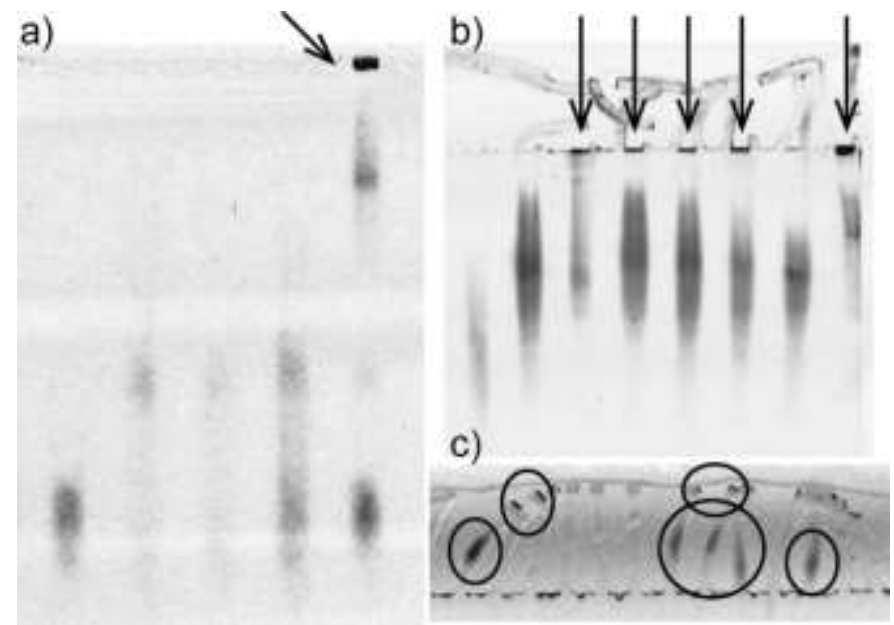

Figure 94. Attempts to obtain evidence of $(\mathbf{s 4 . s 5}){ }_{2} \mathrm{M}(\mathrm{II})$. Native polyacrylamide gels (4\% stacking gel, 8\% running gel) in TBM buffer. a) gel with samples submitted to fast cooling, b) gel with samples submitted to an intermediate cooling and c) Stacking gel (4\%) loaded with samples under slow cooling, indicating still some traces of polymerization.

Evidences of the dimerization for DNA systems carrying multiple modifications pointing outside of the double helix were obtained by Stulz et al., for nucleotides based on terpy group. ${ }^{[30]}$ In the work mentioned, it is not presented results for gel electrophoresis, but AFM results suggested a polymerization where the authors concluded a possible tubular nanostructure. It was also obtained a polymeric DNA structure in the work presented by Sleiman et al., as commented before, where the ruthenium complexes in the end of the strands, upon slow cooling provides a cyclic hybridized DNA structure while under a fast cooling the strands hybridize with different pairs of complementary strands giving then polymeric adducts as final product. ${ }^{[46]}$

The best conditions gathered for our work were repeated and provided another gel (with O`Range Ruler 10-150 bp) were can be established a better interpretation of the obtained spots (Figure 95). 


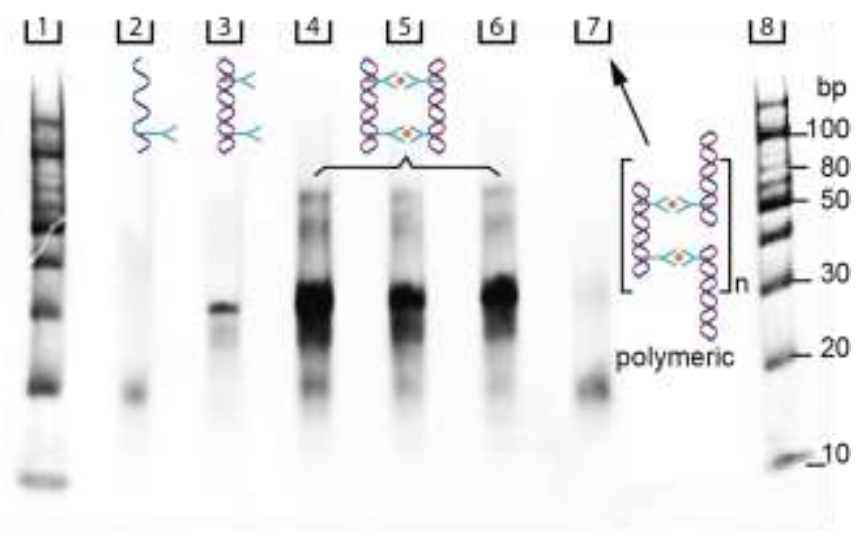

Figure 95. Native 8\% polyacrylamide gel in TBM buffer. Lane 1 and 8: DNA ladder, lane 2: single strand s4, lane 3: double strand s4.s5, lane 4: s4.s5 + 1 eq. $\mathrm{Cu}$ (II) (slow cooling), lane 5: s4.s5 + 1 eq. $\mathrm{Ni}(\mathrm{II})$ (slow cooling), lane 6: s4.s5 + 2 eq. $\mathrm{Cu}(\mathrm{II})$ (slow cooling), lane 7: s4.s5 + 1 eq. $\mathrm{Cu}$ (II) (fast cooling).

In Figure 95 is possible to observe the formation of higher spots for lanes 4,5 and 6 , which indicates the possible formation of metallated-DNA adducts. The spots are faint, which also demonstrated that only a small amount of the samples were converted as dimer-units. It is possible to see two higher spots, not only one, which we attributed to the formation of dimerunits mono and doubly metallated, with the heigth attributed to the mobility between them not so different, as indeed was indicated. The last lane (line 7) gives again evidence of the polymerization of the sample, the material was submitted to a fast cooling and there are only traces of the single strand in the gel. It is not clear why the spots of the duplex s4.s5 for the samples prepared with addition of $M(I I)$ indicated a stronger color. The spot after staining has a mild fluorescence from the artificial nucleotides and after stained it is comprehensible the spots become stronger due to the combination of the fluorescence from the DNA and the fluorescence from the stain (SYBr green stain) producing darker spots after the conversion of the picture for black and white.

Another fact to take into account is, the samples containing metallated DNA (Figure 95 in lanes 4,5 and 6 ) were submitted to a previous slow cooling process before loaded into the gel to obtain as product the metal-DNA dimer as unit instead of the polymerized product. The previous heating process followed by the slow cooling causes a stronger emission of the sample, ${ }^{[67]}$ which in comparison to the other spots (lane 2 and 3, unmetallated and not previously heated) means stronger colored spots. Regarding the blurry and smeared profile around the spot assigned as unmetallated ds-DNA (Figure 95 in lanes 4, 5 and 6) indicated perhaps the formation of denaturated metallated systems, or small metallated adducts with similar mobility to the duplex s4.s5. 


\section{Conclusions and Outlook}

The project presented new types of artificial nucleosides based on trans-chelating ligands sucessfully incorporated into the DNA through solid phase synthesis and its ability to form square-planar complexes upon addition of $\mathrm{M}(\mathrm{II})$. This brings new possibilities of systems with heteroleptic designs ${ }^{[93]}$ and also combination of different metal species which will contribute to the expansion of possible nanoconstructs and aplications.

It was possible to develop a new type of metal-base pairing with an internal trans-chelating ligand and complexation that presents a propeller-shape driven by the DNA helicity providing a great fit into the DNA structure and not causing distortions to the system. It was also possible to study the formation of secondary structure due to interactions of nucleobases and the ligands incorporated, giving to the ligand a high chirality transfer from the DNA. Transchelating ligand can also be submitted to incorporation into DNA with other artificial modifications, such as phenanthroline and terpyridine, allowing the selective metal coordination and bringing systems with mediated charge transport and nanomagnets properties. ${ }^{[39,94]}$ A study for orthogonal self-assembly between the cited ligands with different metal species was also performed and indicated a positive result that can be potentially extended to the DNA environment.

The idea of an artificial ligand with a binding motif pointing outside of the DNA helix was developed with a sucessful synthesis of a trans-chelating ligand completelly linear to the DNA duplex, to avoid deviations and distortions for the structure once established the metal complexation. The syntheses of DNA systems mono and doubly modified were performed and indicated evidences of interduplex cross-link through the metal coordination. It was also possible to obtain monomeric structures, been suported by results of FRET and PAGE for the mono modified system upon excess of addition of $M(I I)$. Preliminary studies of the dimeric units were performed by AFM and it is desired to pursuit great evidences through this method. The system also gave us surprisingly unspecific interactions that led to an unmetallated dimeric structure as could be observed by ESI-MS, bringing the possibilities of systems with incorporation of artificial ligands able to produce interactions beyond the metal coordination to form high ordered DNA constructs. For the future it can be developed the doubly modified system with both modifications in a single strand, with further studies involving the competition between two possible DNA constructs, a cyclic single stranded system closed by metal coordination and the dimeric unit given by the inter cross-link between duplexes. It is 
also expected to explore heteroleptic systems, allowing a more stable formation of dimeric units by interduplex cross-link. A DNA duplex with intercalated artificial nucleotides containing trans-chelating and porphyrine ligands was also imagined with great potential of interaction and charge transport. ${ }^{[95]}$

Potential studies of mimicking and controling movement of the interduplex DNA nanoconstruct through the formation of square-planar complex is also promising. The addition of longer complementary single strand, carrying $T$ bases in one of the sides of the $X$ shape structure would allow the closing of the structure upon addition of $\mathrm{Hg}(\mathrm{II})$ and formation of metal basepairing. ${ }^{[49,57]}$ Such structure could have the opening and closing movement studied by FRET, AFM and PAGE. As observed the research of the incorporation of artificial nucleotides into the DNA provide several possibilities for supramolecular DNA nanodevices with a large number of designs and applications.

\section{Experimental Part}

\subsection{Syntheses of the ligands, complexes, nucleosides and phosphoramidites}

All the products were characterized by nuclear magnetic ressonance (NMR) and electrospray mass spectrometry (ESI-MS). The detail for the solvent used was given in each respective synthesis description. The devices detail are given the following:

NMR spectroscopy:

NMR spectra were measured on a Bruker Avance III 300 (300.13 MHz, auto-sampler BACS 120, $5 \mathrm{~mm}$ BBFO BB-19F/1H ATM z-Grad probe). The chemical shifts for ${ }^{1} \mathrm{H}$ NMR and ${ }^{13} \mathrm{C}$ NMR were given in parts per million, ppm, with the residual solvent signal as: $\mathrm{CD}_{3} \mathrm{CN}$ (1.94 ppm), DMSO$d 6$ (2.50 ppm), $\mathrm{CDCl}_{3}(7.26 \mathrm{ppm})$, DMF (2,95 ppm). For protected DMT ligands, the $\mathrm{CDCl}_{3}$ was previous filtered with $\mathrm{Al}_{2} \mathrm{O}_{3}$ to avoid the acidicity of the solvent promotes cleavage of the protecting group. The chemical shifts for ${ }^{31} \mathrm{P}$ NMR were given relative to $85 \% \mathrm{H}_{3} \mathrm{PO}_{4}$. The 
multiplicities were abbreviated as: singlet (s), doublet (d), doudble doublet (dd), triplet (t), quartet (q), multiplet (m) and $J$ as coupling constant.

Mass Spectrometry:

Electron lonization (EI) mass was performed on a Finnigan MAT 8200 (positive mode, ionization energy $70 \mathrm{eV}$ ) and Electronspray ionization mass (ESI-MS) was performed in a Bruker maXis or MicroTOF mass spectrometers, (positive and negative mode). Concentration, solution composition and other details are given on respective results parts. Calibration performed with low concentrated "Tuning Mix" standard solutionpurchased by Agilent for calibration in the positive as well as in the negative ion mode.

\section{Part 1)}

\section{a) Synthesis of the ligands}

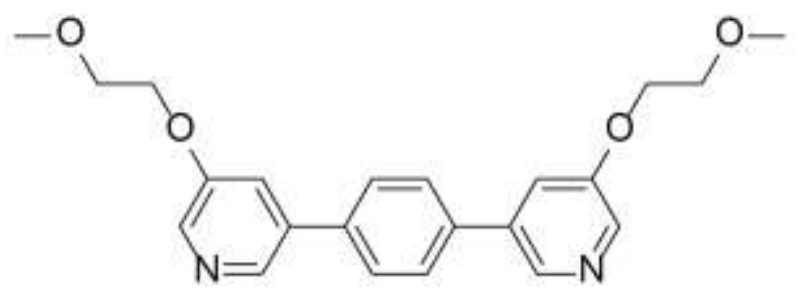

1,4-bis(5-2-methoxyethoxy-pyridine-3-yl)benzene , L4: 3-Bromo-5-(2-methoxyethoxy)pyridine (100 mg, $431 \mu \mathrm{mol}$ ), 1,4-benzenediboronic acid dipinacol ester (71 mg, $215 \mu \mathrm{mol}), \mathrm{K}_{2} \mathrm{CO}_{3}(297$ $\mathrm{mg}, 2.15 \mathrm{mmol})$ and $\mathrm{Pd}(\mathrm{dppf}) \mathrm{Cl}_{2}(9.6 \mathrm{mg}, 11.7 \mu \mathrm{mol})$ were submitted to a protected atmosphere with a mixture of toluene $(2.0 \mathrm{~mL})$ and water $(1.0 \mathrm{~mL})$ and degassed. After the system was stirred at $85^{\circ} \mathrm{C}$ for $96 \mathrm{~h}$. The raw material was poured into water and washed $(4 \mathrm{x})$ with $25 \mathrm{~mL}$ of diethylether. The organic layer was filtered with celite and the solution was evaporated in vaccum producing a brown solid as product $(75.0 \mathrm{mg}, 197 \mu \mathrm{mol})$. Yield: $91 \% .{ }^{1} \mathbf{H}$ NMR $\left(300 \mathrm{MHz}, \mathrm{CD}_{3} \mathrm{CN}\right): \delta=3.38(\mathrm{~s}, 6 \mathrm{H}), 3.81-3.67(\mathrm{~m}, 4 \mathrm{H}), 4.26(\mathrm{~m}, J=1.7 \mathrm{~Hz}, 4 \mathrm{H}), 7.58(\mathrm{dd}$, $J=2.7,2.0 \mathrm{~Hz}, 2 \mathrm{H}), 7.82(\mathrm{~s}, 4 \mathrm{H}), 8.29(\mathrm{~d}, J=2.8 \mathrm{~Hz}) 8.51(\mathrm{~s}, 2 \mathrm{H})$. ESI HRMS: $\mathrm{m} / \mathrm{z}[\mathrm{M}+\mathrm{H}]^{+}$; calc. for $\mathrm{C}_{22} \mathrm{H}_{26} \mathrm{~N}_{2} \mathrm{O}_{4}=381,1809$; found: 381.1808 . 


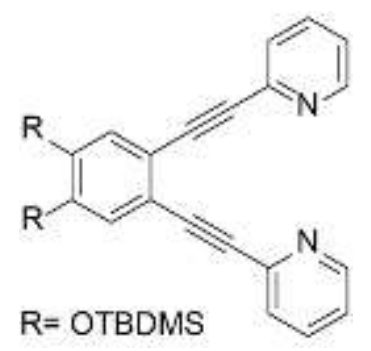

1,2-bis(tert-butyldimethylsilyloxy)-4,5-bis(pyridine-2-ylethinyl)benzene, L2c: 4,5-bis(tertbutyldimethylsilyloxy)-1,2-diiodobenzene ( $246 \mathrm{mg}, 423 \mu \mathrm{mol})$, Cul $(5.4 \mathrm{mg}, 28 \mu \mathrm{mol})$ and $\mathrm{Pd}\left(\mathrm{PPh}_{3}\right)_{2} \mathrm{Cl}_{2}$ (31.7 mg, $\left.45 \mu \mathrm{mol}\right)$ were added in a flask under protected atmosphere and degassed after addition of dry $\mathrm{Et}_{3} \mathrm{~N}(8 \mathrm{~mL})$. After it, 2-ethynyl-pyridine (108.9 mg, $\left.1.06 \mathrm{mmol}\right)$ was added and the mixture stirred at $60^{\circ} \mathrm{C}$ for $2 \mathrm{~h}$. The mixture was evaporated in vacuum and the dark brown oil produced was dissolved in dichloromethane followed by column chromatography on silica gel (hexane/EtOAc 1:1) for purification. The final product obtained as a brown oil $(71.5 \mathrm{mg}, 132 \mu \mathrm{mol})$. Yield: $32 \% .{ }^{1} \mathrm{H}$ NMR $\left(300 \mathrm{MHz}, \mathrm{CD}_{3} \mathrm{CN}\right): \delta=0.30(\mathrm{~s}, 12 \mathrm{H}, \mathrm{Si}-$ $\left.\mathrm{CH}_{3}\right), 1.02(\mathrm{~s}, 18 \mathrm{H}, t B u), 7.15(\mathrm{~s}, 2 \mathrm{H}), 7.34$ (ddd, $\left.J=7.3,4.9,1.6 \mathrm{~Hz}, 2 \mathrm{H}\right), 7.81-7.70(\mathrm{~m}, 4 \mathrm{H})$, 8.64 (ddd, $J=4.9,1.8,1.0 \mathrm{~Hz}, 2 \mathrm{H}$ ). ESI HRMS: $\mathrm{m} / \mathrm{z}[\mathrm{M}+\mathrm{H}]^{+}$; calc. For $\mathrm{C}_{32} \mathrm{H}_{43} \mathrm{~N}_{2} \mathrm{O}_{2} \mathrm{Si}_{2}=541.2701$; found: 541.2698 .<smiles>O=C1c2cc(I)c(I)cc2C(=O)N1c1ccc(Br)cc1</smiles>

$\boldsymbol{N}$-(4-bromophenyl)-4,5-diiodophthalimide: Two parts of the solid 4-bromo-aniline were combined with 1 part of the precursor 4,5-diiodophthalic anhydride at $180^{\circ} \mathrm{C}$ for 5 minutes producing a brown syrup as crude material that was submitted to column chromatography on silica gel (DCM/Hexane 10:1) to yield (23\%) a white solid (316 mg, $570 \mu \mathrm{mol}$ ) as final product. ${ }^{1} \mathrm{H}$ NMR $\left(300 \mathrm{MHz}, \mathrm{CDCl}_{3}\right): \delta=7.32(\mathrm{~d}, J=8.7 \mathrm{~Hz}, 2 \mathrm{H}), 7.63(\mathrm{~d}, J=8.7 \mathrm{~Hz}, 2 \mathrm{H}), 8.43(\mathrm{~s}, 2 \mathrm{H})$. ESI HRMS: $\mathrm{m} / \mathrm{z}[\mathrm{M}+\mathrm{Na}]^{+}$; calc. For $\mathrm{C}_{14} \mathrm{H}_{6} \mathrm{Brl}_{2} \mathrm{NO}_{2} \mathrm{Na}=575.7563$; found: 575.7556 . This compound was used as starting material for the next ligand (L2b), synthesized. 


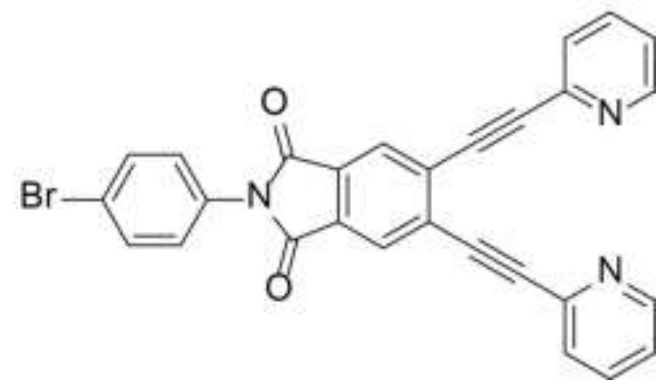

N-(4-bromophenyl)-4,5-bis(pyridine-2-ylethynyl)phthalimide, L2b: $\quad N$-(4-bromophenyl)-4,5diiodophthalimide (228 mg, $412 \mu \mathrm{mol})$, Cul $(4.6 \mathrm{mg}, 24.1 \mu \mathrm{mol})$ and $\mathrm{PdCl}_{2}\left(\mathrm{PPh}_{3}\right)_{2}(17 \mathrm{mg}, 24.1$ $\mu \mathrm{mol})$ were combined in protected athmosphere following by addition of dry THF $(12 \mathrm{~mL})$ and the system degassed. After it it was added 2-ethynyl-pyridine (104 mg, $101 \mu \mathrm{mol}$ ) and $\mathrm{Et}_{3} \mathrm{~N}$ (5eq.). The reaction was stirrred overnight at room temperature and the solvents evaporated by vaccum. The crude material was purified by column chromatography on silica gel $\left(\mathrm{CHCl}_{3} / \mathrm{MeOH} 70: 1\right)$ yielding a yellow solid (257 mg, $\left.509 \mu \mathrm{mol}\right)$ as final product (62\%). ${ }^{1} \mathrm{H}$ NMR (300 MHz, $\left.\mathrm{CD}_{3} \mathrm{CN}\right): \delta=7.46-7.39(\mathrm{~m}, 4 \mathrm{H}), 7.74-7.70(\mathrm{~m}, 2 \mathrm{H}), 7.88-7.85(\mathrm{~m}, 4 \mathrm{H}), 8.21(\mathrm{~s}, 2 \mathrm{H})$, 8.69 (dt, $J=4.9,1.4 \mathrm{~Hz}, 2 \mathrm{H}$ ). ESI HRMS: $\mathrm{m} / \mathrm{z}[\mathrm{M}+\mathrm{H}]^{+}$; calc. for $\mathrm{C}_{28} \mathrm{H}_{16} \mathrm{~N}_{3} \mathrm{BrO}_{2}=504.0328$; found: 504.0342 .

Synthesis of ligand $\mathbf{L} \mathbf{2}$ and $\mathbf{L} 5$ were performed according to literature. ${ }^{[78,83]}$

\section{b) Synthesis of the metal complexes:}

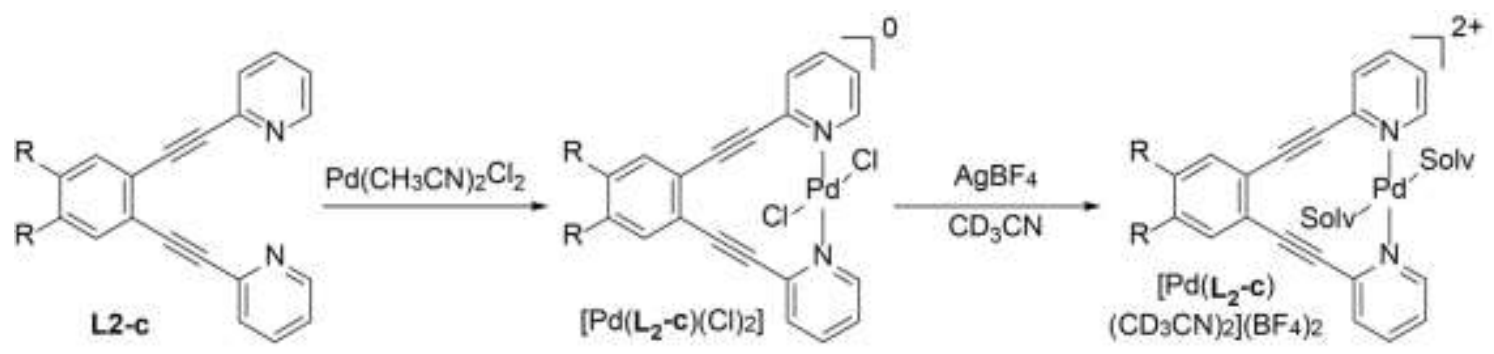

[Pd(L2-2c) $\left.\left(\mathrm{CD}_{3} \mathrm{CN}\right)_{2}\right]\left(\mathrm{BF}_{4}\right)_{2}$ : ligand L2-2c $(45 \mu \mathrm{mol})$ were added into $\mathrm{CH}_{2} \mathrm{Cl}_{2} \quad(5 \mathrm{~mL})$ with $\mathrm{PdCl}_{2}\left(\mathrm{CH}_{3} \mathrm{CN}\right)_{2}(62.3 \mu \mathrm{mol})$. The mixture was stirred at room temperature for $1 / 2 \mathrm{~h}$ and the solvent evaporated in vacuum. The crude product was redissolved in dichloromethane and purified by column chromatography on silica gel $\left(\mathrm{CH}_{2} \mathrm{Cl}_{2} / \mathrm{MeOH}, 20: 1\right)$ to yield $(50-52 \%$ respectively) the complexes $\left[\mathrm{PdCl}_{2}(\mathbf{L 2 - 2 \mathbf { C }})\right]$ as a solid $(23 \mu \mathrm{mol})$. The exchange of chlorides for acetonitrile ligands was performed upon addition of $\mathrm{AgBF}_{4}$ solution in deuterated acetonitrile. A $2.8 \mathrm{mM}$ solution of $\left[\mathrm{PdCl}_{2}(\mathbf{L 2}-\mathbf{2} \mathbf{C})\right]$ in $\mathrm{CD}_{3} \mathrm{CN}(6.3 \mu \mathrm{mol}, 2.25 \mathrm{~mL})$ was prepared and combined in 1:2 equivalence to a $11.24 \mathrm{mM}$ solution of $\mathrm{AgBF}_{4}$ in $\mathrm{CD}_{3} \mathrm{CN}(23.6 \mu \mathrm{mol}, 2.10 \mathrm{~mL})$. The mixture 
was stirred manually and kept in a dark space for $1 \mathrm{~h}$ at room temperature. The $\mathrm{AgCl}$ precipitated as a white-grey solid. ${ }^{1} \mathbf{H}$ NMR $\left[\mathrm{Pd}\left(\mathrm{L2}_{2}\right)\left(\mathrm{CD}_{3} \mathrm{CN}\right)_{2}\right]\left(\mathrm{BF}_{4}\right)_{2}\left(300 \mathrm{MHz}, \mathrm{CD}_{3} \mathrm{CN}\right): \delta=7.64$ (ddd, 7.5, 5.8 1.6 Hz, 2 H), 7.71 (dd, 5.8, 3.3 Hz, 2 H), 7.96-7.88 (m, 4 H, H3), 8.09 (td, 7.8, 1.5 $\mathrm{Hz}, 2 \mathrm{H}), 8.90$ (ddd, 5.8, 1.5, 0.7 Hz, $2 \mathrm{H}) .{ }^{1} \mathrm{H}$ NMR $\left[\mathrm{Pd}(\mathrm{L} 2 c)\left(\mathrm{CD}_{3} \mathrm{CN}\right)_{2}\right]\left(\mathrm{BF}_{4}\right)_{2}\left(300 \mathrm{MHz}, \mathrm{CD}_{3} \mathrm{CN}\right): \delta=$ 0.35 (s, $12 \mathrm{H}, \mathrm{Si}-\mathrm{CH} 3$ ), 1.06 (s, $18 \mathrm{H}, t \mathrm{Bu}$ ), 7.45 (s, $2 \mathrm{H}$ ), 7.62 (ddd, $2 \mathrm{H}$ ), 7.89 (d, $2 \mathrm{H}$ ), 8.07 (td, 2 $H), 8.89(m, 2 H)$.
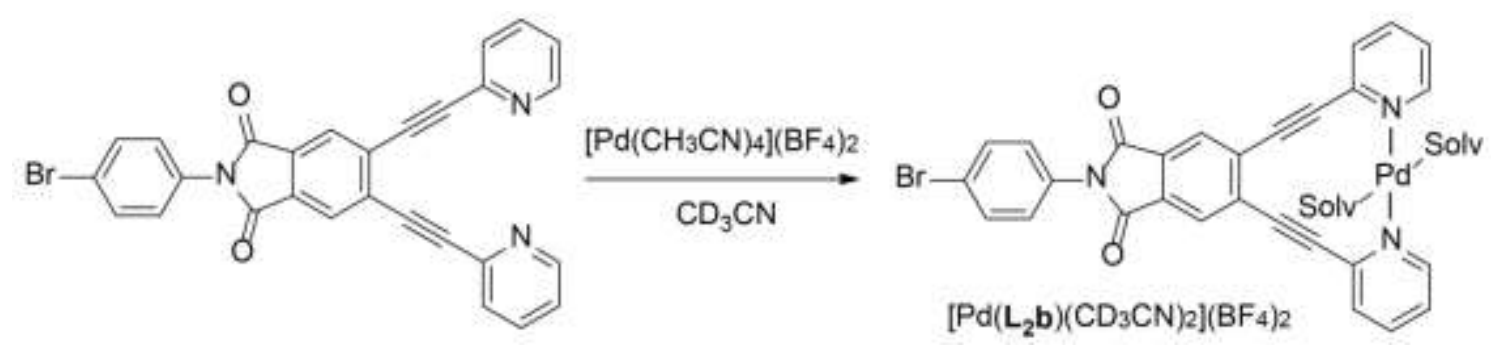

$\left[\mathrm{Pd}(\mathrm{L} 2 \mathrm{~b})\left(\mathrm{CD}_{3} \mathrm{CN}\right)_{2}\right]\left(\mathrm{BF}_{4}\right)_{2}:\left[\mathrm{Pd}\left(\mathrm{CH}_{3} \mathrm{CN}\right)_{4}\right]\left(\mathrm{BF}_{4}\right)_{2}(12 \mu \mathrm{mol})$ and L2b $(10 \mu \mathrm{mol})$ were combined into $\mathrm{CD}_{3} \mathrm{CN}(1 \mathrm{~mL})$ to give a $1 \mathrm{mM}$ solution of the monomer complex $\left[\mathrm{Pd}(\mathrm{L} 2 \mathbf{b})\left(\mathrm{CD}_{3} \mathrm{CN}\right)_{2}\right]\left(\mathrm{BF}_{4}\right)_{2}$ ${ }^{1} \mathrm{H}$ NMR $\left(300 \mathrm{MHz}, \mathrm{CD}_{3} \mathrm{CN}\right.$ ): $\delta=7.44-7.39(\mathrm{~m}, 2 \mathrm{H}), 7.76-7.70(\mathrm{~m}, 2 \mathrm{H}), 7.82$ (ddd, $J=7.65 .9,1.6$ $\mathrm{Hz}, 2 \mathrm{H}$ ), 8.07 (ddd, $J=7.9,1.6,0.7 \mathrm{~Hz}, 2 \mathrm{H}$ ), 8.24 (ts, $J=7.8,1.5 \mathrm{~Hz}, 2 \mathrm{H}), 8.46(\mathrm{~s}, 2 \mathrm{H}), 9.04$ (ddd, $J=5.9,1.5,0.7 \mathrm{~Hz}, 2 \mathrm{H}$ ).

\section{c) Synthesis of the rings:}

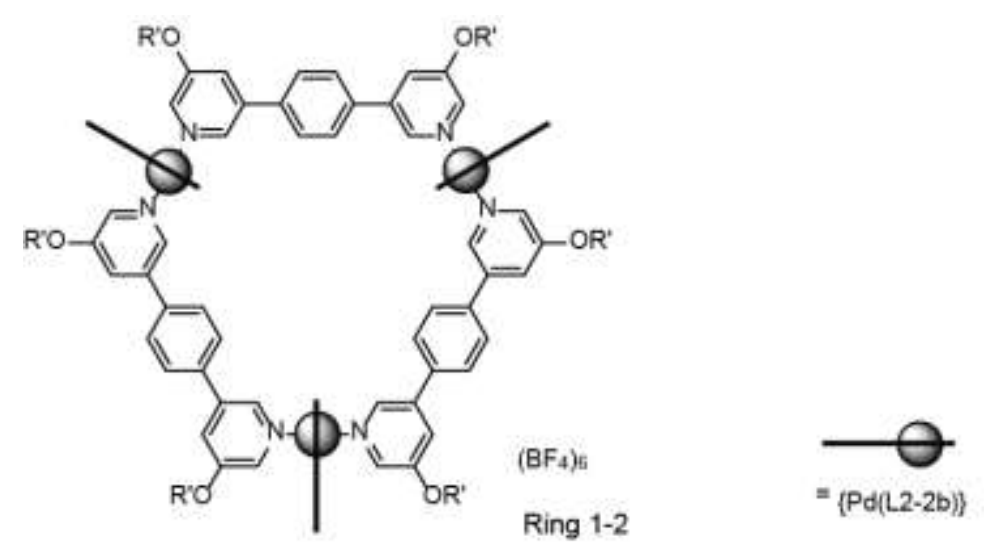

RING1: A 7.0 M solution of $\mathbf{L} 4(50 \mu \mathrm{L})$ in $\mathrm{CD}_{3} \mathrm{CN}$ was combined in a $1: 1$ ratio to a $0.7 \mathrm{M}$ solution of $\left[\mathrm{Pd}(\mathrm{L2})\left(\mathrm{CD}_{3} \mathrm{CN}\right)_{2}\right]^{2+}(500 \mu \mathrm{L})$ in $\mathrm{CD}_{3} \mathrm{CN}$. The mixture was well stirred manually and left at room temperature for 10 minutes to form RING1. ${ }^{1} \mathrm{H}$ NMR $\left(300 \mathrm{MHz}, \mathrm{CD}_{3} \mathrm{CN}\right): \delta=3.22(\mathrm{~s}, 18 \mathrm{H})$, 3.48-3.54 (m, $12 \mathrm{H}), 4.09-4.15(\mathrm{~m}, 12 \mathrm{H}), 7.83-7.77(\mathrm{~m}, 12 \mathrm{H})$, 7.91-7.96 (m, $6 \mathrm{H})$, 7.96-7.91 (m, $6 \mathrm{H}), 8.02-7.98(\mathrm{~m}, 18 \mathrm{H}), 8.07(\mathrm{td}, J=7.8,1.5 \mathrm{~Hz}), 8.22-8.17(\mathrm{~m}, 6 \mathrm{H}), 8.93(\mathrm{~d}, J=4.2 \mathrm{~Hz}, 6 \mathrm{H})$, 
$9.29(\mathrm{~d}, J=3 \mathrm{~Hz}, 6 \mathrm{H}), \quad 9.57(\mathrm{~d}, 6.3 \mathrm{~Hz}, 6 \mathrm{H})$. ESI HRMS: $\mathrm{m} / \mathrm{z}[\mathrm{M}+\mathrm{H}]^{+}$; calc. for $\left[\mathrm{C}_{126} \mathrm{H}_{108} \mathrm{~F}_{8} \mathrm{~N}_{12} \mathrm{O}_{12} \mathrm{Pd}_{3} \mathrm{~B}_{2}\right]^{4+}=618.6356$; found: 618.6391 .

RING2 A 3 mM solution of $\mathbf{L} 4(1.03 \mathrm{~mL})$ in $\mathrm{CD}_{3} \mathrm{CN}$ was combined with $10.3 \mathrm{mM}$ a solution of $\left[\mathrm{Pd}(\mathbf{L} 2 \mathbf{b})\left(\mathrm{CD}_{3} \mathrm{CN}\right)_{2}\right]^{2+}(300 \mu \mathrm{L})$ in $\mathrm{CD}_{3} \mathrm{CN}$. The mixture was manually stirred and left at room temperature for 10 minutes to form RING2. ${ }^{1} \mathbf{H}$ NMR $\left(300 \mathrm{MHz}, \mathrm{CD}_{3} \mathrm{CN}\right): \delta=3.21(\mathrm{~s}, 18 \mathrm{H})$, 3.54-3.51 (m, 12 H), 4.13- $4.10(m, 12 H), 7.44(d, J=8.7$ Hz, 6 H), $7.61(d, J=8.4$ Hz, 6 H), 7.78$7.73(\mathrm{~m}, 12 \mathrm{H}), 7.88(\mathrm{~s}, 12 \mathrm{H}), 8.11-7.99(\mathrm{~m}, 12 \mathrm{H}), 8.68(\mathrm{~s}, 6 \mathrm{H}), 8.81(\mathrm{~d}, J=2.5 \mathrm{~Hz}, 6 \mathrm{H}), 9.24-$ $9.21(\mathrm{~m}, 6 \mathrm{H}), 9,49(\mathrm{~d}, J=5.9 \mathrm{~Hz}, 6 \mathrm{H})$.
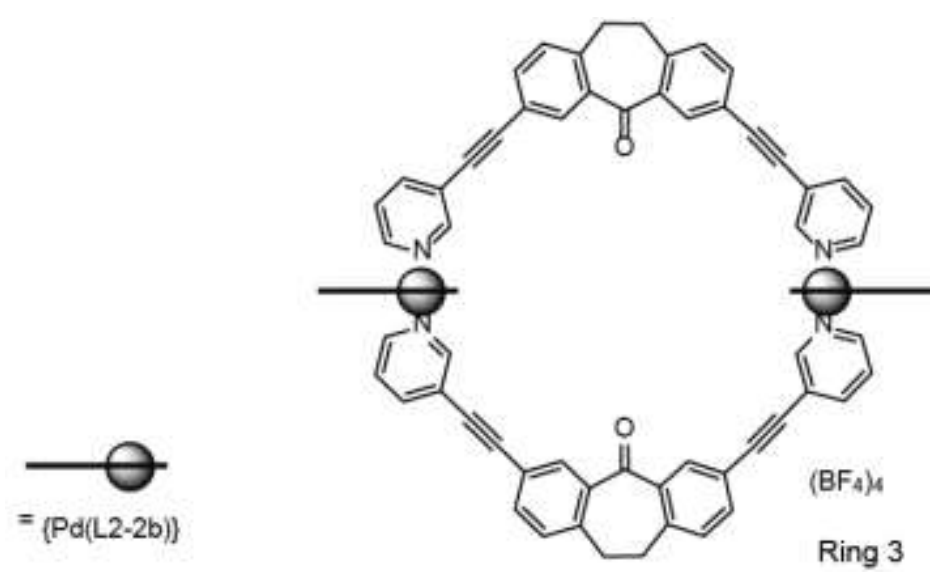

RING3: A 2.8 M solution of $\mathbf{L 5}^{\left[{ }^{[83]}\right.}(250 \mu \mathrm{L})$ in $\mathrm{CD}_{3} \mathrm{CN}$ was combined to a $0.7 \mathrm{M}$ solution of $\left[\mathrm{Pd}(\mathrm{L2})\left(\mathrm{CD}_{3} \mathrm{CN}\right)_{2}\right]^{2+}$ in $\mathrm{CD}_{3} \mathrm{CN}(1 \mathrm{~mL})$. The mixture was manually stirred and left for 5 minutes at room temperature to form RING3. ${ }^{1} \mathbf{H}$ NMR $\left(300 \mathrm{MHz}, \mathrm{CD}_{3} \mathrm{CN}\right): \delta=3.28(\mathrm{~s}, 4 \mathrm{H}), 7.41(\mathrm{~d}, 4 \mathrm{H})$, $7.53(\mathrm{ddd}, J=8.1,5.8,0.7 \mathrm{~Hz}, 4 \mathrm{H}), 7.76-7.67(\mathrm{~m}, 8 \mathrm{H}), 7.95-7.86(\mathrm{~m}, 8 \mathrm{H})$, 8.08-7.99 $(\mathrm{m}, 8 \mathrm{H}$, H4), 8.18-8.10 (m, 4 H), 9.16-9.12 (m, 4 H), 9.26 (dd, J = 1.6, 0.9 Hz), 9.42 (m, 4 H). ESI MS: m/z $[\mathrm{M}+\mathrm{H}]^{+}$; calc. for $\left.\left[\mathrm{C}_{98} \mathrm{H}_{60} \mathrm{~N}_{8} \mathrm{O}_{2} \mathrm{Pd}_{2} \mathrm{BF}_{4}\right)\right]^{3+}=560.4324$; found: 560.4133 .

\section{Part 2)}<smiles>OCCOc1ccc(Br)nc1</smiles>

2-bromo-5-hydroxyethoxypyridine 2: 2-bromo-5-hydroxypyridine $\quad(4 \mathrm{~g}, \quad 23 \mathrm{mmol}), \quad 2-$ chloroethanol $(7.4 \mathrm{~g}, 92.0 \mathrm{mmol})$ and $\mathrm{K}_{2} \mathrm{CO}_{3}(7.94 \mathrm{~g}, 57.5 \mathrm{mmol})$ were submitted to reflux in acetonitrile overnight. By filtration is removed a white salt and washed with acetonitrile. A thick yellowish oil is obtained from the colorless solution after been reduced. The oil was Column chromatography purification on silica gel $\left(\mathrm{CHCl}_{3} / \mathrm{MeOH} 10: 1\right)$ was performed and a 
colorless solution was reduced until obtain an yellowish oil which was submitted to vacuum to produce a white solid $(4.7 \mathrm{~g}, 21.7 \mathrm{mmol}) .{ }^{1} \mathrm{H}$ NMR $\left(300 \mathrm{MHz}, \mathrm{CDCl}_{3}\right): \delta=8.06(\mathrm{~d}, 1 \mathrm{H}, J=2.4 \mathrm{~Hz})$ $7.39(\mathrm{~d}, 1 \mathrm{H}, J=6 \mathrm{~Hz}), 7.15-7.14(\mathrm{dd}, 1 \mathrm{H}, J=2.4,6.6 \mathrm{~Hz}), 4.12-4.10(\mathrm{t}, 2 \mathrm{H}, J=6.6 \mathrm{~Hz}), 4.01-3.99$ $(\mathrm{t}, 2 \mathrm{H}, J=6.9 \mathrm{~Hz}) .{ }^{13} \mathrm{C}$ NMR $\left(75 \mathrm{MHz}, \mathrm{CDCl}_{3}\right): \delta=154.86,137.47,132.50,128.51,125.31,70.19$, 61.23. ESI HRMS: $\mathrm{m} / \mathrm{z}[\mathrm{M}+\mathrm{H}]^{+}$; calc. for $\mathrm{C}_{7} \mathrm{H}_{9} \mathrm{BrNO}_{2}$ : 217.9811; found: 217.9814 .

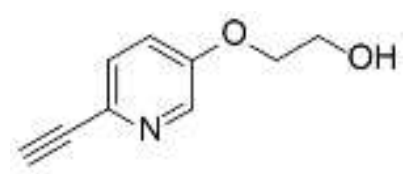

2-ethynyl-5-hydroxyethoxypyridine, 3: 2 (4.5 g, $20.6 \mathrm{mmol}), \mathrm{PdCl}_{2}\left(\mathrm{PPh}_{3}\right)_{2}(721 \mathrm{mg}, 1 \mathrm{mmol})$ and Cul (196 $\mathrm{mg}, 1 \mathrm{mmol}$ ) were submitted to a protected atmosphere, been evacuated and filled with $\mathrm{N}_{2}$ previous to addition of dry THF ( 10 mL). The solution was degassed (3x) before addition of trimethylsilyl-acetylene $(2.43 \mathrm{~g}, 24.8 \mathrm{mmol})$ and $\mathrm{Et}_{3} \mathrm{~N}(2 \mathrm{~mL}, 14.3 \mathrm{mmol})$ under protected atmosphere. The system stirred overnight at room. The reaction mixture was concentrated and the raw material submitted to column chromatography on silica gel $\left(\mathrm{CHCl}_{3} / \mathrm{MeOH} 10: 1\right)$ for purification. Light brown solid $(605 \mathrm{mg}, 3.73 \mathrm{mmol})$, yield: $39 \%$ was obtained as product. ${ }^{1} \mathrm{H}$ NMR $\left(400 \mathrm{MHz}, \mathrm{CDCl}_{3}\right): \delta=8.31(\mathrm{~d}, 1 \mathrm{H}, \mathrm{J}=3.2 \mathrm{~Hz}), 7.46(\mathrm{~d}, 1 \mathrm{H}, \mathrm{J}=$ $9.2 \mathrm{~Hz}$ ), $7.21(\mathrm{dd}, 1 \mathrm{H}, J=4.0 \mathrm{~Hz}, 11.6 \mathrm{~Hz}), 4.17(\mathrm{t}, 2 \mathrm{H}, J=11.6 \mathrm{~Hz}), 4.02(\mathrm{t}, 2 \mathrm{H}, J=12.0 \mathrm{~Hz}$ ), 3.09 $(\mathrm{s}, 1 \mathrm{H}) .{ }^{13} \mathrm{C}$ NMR $\left(75 \mathrm{MHz}, \mathrm{CDCl}_{3}\right): \delta=154.86,138.47,132.50,128.29,121.33,82.46,76.33$, 69.95, 61.33 ESI HRMS: $\mathrm{m} / \mathrm{z}[\mathrm{M}+\mathrm{H}]^{+}$; for $\mathrm{C}_{9} \mathrm{H}_{10} \mathrm{NO}_{2}$ calc: 164.0643 found: 164.1067 .

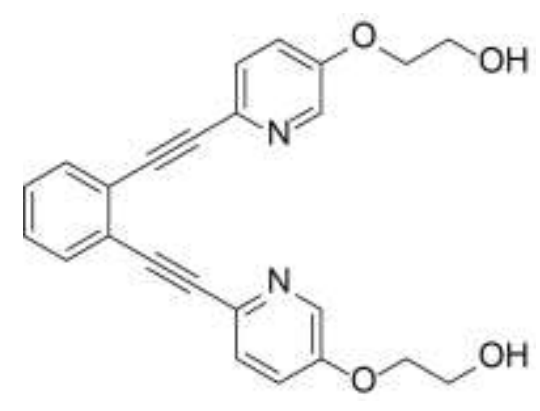

1,2-bis(2-ethynyl-5-hydroxyethoxypyridine)benzene, 4: 3 (605 mg, $3.71 \mathrm{mmol}$ ), Cul (16 mg, $0.84 \mathrm{mmol}$ ), $\mathrm{PdCl}_{2}\left(\mathrm{PPh}_{3}\right)_{2}(59 \mathrm{mg}, 0.84 \mathrm{mmol}), 1,2$-diiodobenzene $(559 \mathrm{mg}, 1.69 \mathrm{mmol})$, were submitted to a protected atmosphere, evacuated and filled with $\mathrm{N}_{2}$ previous to addition of dry DMF ( $8 \mathrm{~mL}$ ). The system was degassed under $\mathrm{N}_{2}$ atmosphere and $\mathrm{Et}_{3} \mathrm{~N}(1.5 \mathrm{~mL}, 10.7 \mathrm{mmol})$ added. The reaction was heated at $120^{\circ} \mathrm{C}$ and stirred overnight. The raw material was concentrated in vacuo and then purified by column chromatography on silica gel $\left(\mathrm{CHCl}_{3} / \mathrm{MeOH}\right.$ 100:1) giving as product a brown solid $(339 \mathrm{mg}, 0.85 \mathrm{mmol})$, yield: $50 \% .{ }^{1} \mathrm{H}$ NMR $(300 \mathrm{MHz}$, $\left.\mathrm{CDCl}_{3}\right): \delta=8.36(\mathrm{~d}, 2 \mathrm{H}, J=2.7 \mathrm{~Hz}$ ), 7.65-7.59 (m, $4 \mathrm{H}), 7.35-7.32(\mathrm{q}, 2 \mathrm{H}, J=9.0 \mathrm{~Hz}), 7.22-7.18$ 
(dd, $2 \mathrm{H}, J=3.0 \mathrm{~Hz}, 8.7 \mathrm{~Hz}$ ), 4.19-4.16 (t, $2 \mathrm{H}, J=9.0 \mathrm{~Hz}$ ), 4.03-4.00 (t, $2 \mathrm{H}, J=8.7 \mathrm{~Hz}) .{ }^{13} \mathrm{C} \mathrm{NMR}$ $\left(75 \mathrm{MHz}, \mathrm{CDCl}_{3}\right): \delta=154.86,139.00,133.91,131.83,129.15,128.58,124.57,121.29$, 93.29, 85.65, 70.25, 59.41 ESI HRMS: $\mathrm{m} / \mathrm{z}[\mathrm{M}+\mathrm{H}]^{+}$; for $\mathrm{C}_{24} \mathrm{H}_{21} \mathrm{~N}_{2} \mathrm{O}_{4}$ calc: 401.1496 found: 401.1482 .

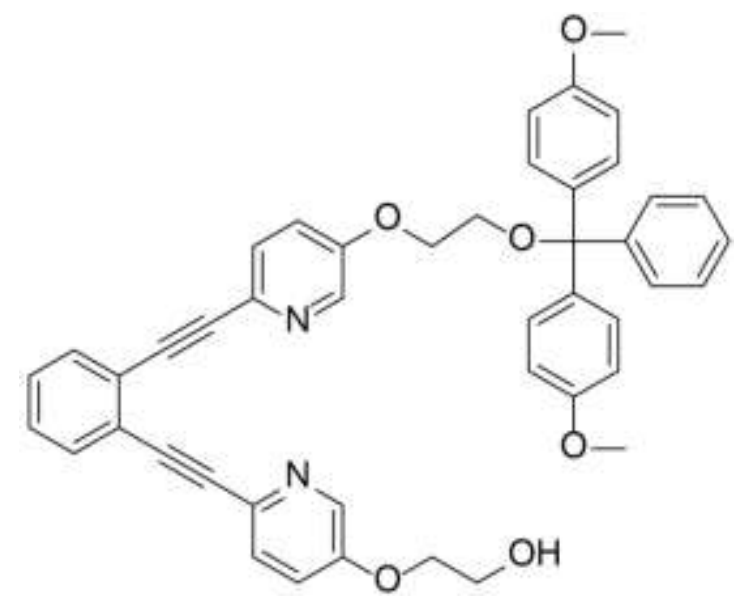

1-(2-ethynyl-5-hydroxyethoxy)-2-(2-ethynyl-5-(4,4'-dimethoxytrityl)oxyethoxy)-benzene, 5 : 4 (450 mg, $1.12 \mathrm{mmol})$, dry pyridine $(10 \mathrm{~mL}$ ) and molecular sieves (3 $\AA$ ) were submitted to protected atmosphere in a flask previously evacuated $(3 x)$ and degassed with $N_{2}(3 x)$ after solvent addition. The reaction stirred for $1.5 \mathrm{~h}$ at room temperature. After it, DMT-Cl (457 mg, $1.35 \mathrm{mmol}$ ) was added under protected atmosphere and stirred for more half an hour when DMAP (1 mg, $8.2 \mu \mathrm{mol}$ ) was added, also under protected atmsphere and left to stir at room temperature overnight. The material was concentrated in vacuo and the raw poduct was submitted to column chromatography on silica gel $\left(\mathrm{CHCl}_{3} / \mathrm{MeOH} 40: 1+0.1 \% \mathrm{py}\right)$ for purification. The product obtained is an oil that yielded a solid foam after submitted to vaccum, (387 mg, $0.55 \mathrm{mmol}) .{ }^{1} \mathrm{H}$ NMR (300 MHz, CDCl 3 ): $\delta=3.36(\mathrm{~d}, J=3.0 \mathrm{~Hz}, 2 \mathrm{H}$ ), 7.64-7.57 $(\mathrm{m}, 4 \mathrm{H}), 7.49-7.44(\mathrm{~m}, 5 \mathrm{H}), 7.36-7.31(\mathrm{~m}, 10 \mathrm{H}), 7.23-7.19(\mathrm{~m}, 2 \mathrm{H}), 4.23-4.16(\mathrm{~m}, 4 \mathrm{H}), 4.14-$ $4.10(\mathrm{~m}, 2 \mathrm{H}), 4.00-3.97(\mathrm{~m}, 2 \mathrm{H}), 3.80\left(\mathrm{~s}, 6 \mathrm{H}, 2 \mathrm{x}-\mathrm{OCH}_{3}\right) .{ }^{13} \mathrm{C} \mathrm{NMR}\left(75 \mathrm{MHz}, \mathrm{CDCl}_{3}\right): \delta=158.65$, $154.88,154.48,138.84,138.52,136.04,132.13,129.27,128.51,128.29,128.01,127.90$, $127.00,125.62,125.54,121.50,121.35,113.29,92.77,92.65,87.21,86.46,83.33,69.89,68.34$, 62.36, 61.35, 55.39 ESI HRMS: $\mathrm{m} / \mathrm{z}[\mathrm{M}+\mathrm{H}]^{+}$; calc. for $\mathrm{C}_{45} \mathrm{H}_{39} \mathrm{~N}_{2} \mathrm{O}_{6}: 703.2803$ found: 703.2785. 


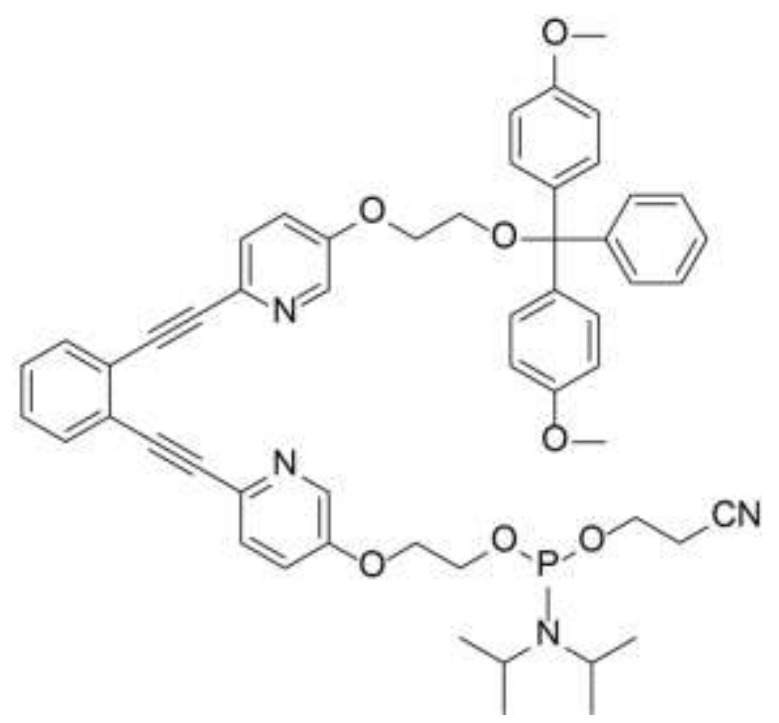

1-(2-ethynyl-5-hydroxyethoxy-1-ol)-2-(2-ethynyl-5-(4,4'-dimethoxytrityl)oxyethoxy)-

benzene-phosphoramidite, 6: $5(119 \mathrm{mg}, 0.17 \mathrm{mmol})$ and dry THF were placed in a flask and co-evaporated under vacuum (3x). After it some molecular sieves $3 \AA$ were added with more dry THF and the system was degassed. (iPr) $)_{2} \mathrm{NEt}(148 \mu \mathrm{L}, 0.85 \mathrm{mmol})$ and $\left(\mathrm{Pr}_{2} \mathrm{~N}\right)\left(\mathrm{OCH}_{2} \mathrm{CH}_{2} \mathrm{CN}\right) \mathrm{PCl},(114 \mu \mathrm{L}, 0.51 \mathrm{mmol})$ were added after half an hour and the mixture was left stirring for around $1 \mathrm{~h}$. The solvent was reduced under vaccum to about $0.5 \mathrm{~mL}$ and cooled with ice bath previous to additon of $2 \mathrm{~mL}$ dry hexane. A milky hexane solution containing impurities and salts was obtained and removed with a needle. The product was precipitated in the flask and left to dry in vacuo yielding a yellowish solid product $(137.6 \mathrm{mg}$, $0.152 \mathrm{mmol}$ ). Estimated yield: $90 \%$. Since the product stability at room temperature and without protected atmosphere is very low, there are no ${ }^{1} \mathrm{H} N M R,{ }^{13} \mathrm{C} N M R$ or ${ }^{31} \mathrm{P} N M R$ data available and the material has to be used for DNA SPS right after synthesized.

\section{Unmodified oligonucleotides:}

Unmodified oligonucleotides s1* and $\mathbf{s 2}$ were purchased by Biomers.net $\mathrm{GmbH}$ in aqueous solution with concentration $200.0 \mathrm{pmol} / \mu \mathrm{l}$ and purified by HPLC. Sequences for both strands, $\mathbf{s 1 *}$ and $\mathbf{s} \mathbf{2}^{*}$, are respective to the sequence for modified strands, containing $d T$ instead of $X$ for $s 1^{*}$ and $d A$ for $s 2^{*}$. 
Part 3)<smiles>Brc1cc(I)c(I)cc1Br</smiles>

1,2-dibromo-4,5-diiodobenzene, 1: Concentrated $\mathrm{H}_{2} \mathrm{SO}_{4}(160 \mathrm{~mL})$ was added into a flask with $\mathrm{I}_{2}(3.62 \mathrm{~g}, 14.3 \mathrm{mmol})$ and $\mathrm{KI}(1.52 \mathrm{~g}, 7.12 \mathrm{mmol})$ and stirred at $40{ }^{\circ} \mathrm{C}$ for $1.5 \mathrm{~h}$. The solution was left to cool down until $0^{\circ} \mathrm{C}$ and kept in such temperature to add slowly 1,2-dibromo-benzene $(4.20 \mathrm{~g}, 17.8 \mathrm{mmol})$. The mixture was kept at $0^{\circ} \mathrm{C}$ and stirring for $4 \mathrm{~h}$. Poured crushed ice was added to the mixture and the white precipitate was filtered. The white/pink solid was washed with water $\left(200 \mathrm{~mL}\right.$ ) before be redissolved in $\mathrm{CHCl}_{3}$ giving a purple solution. The solution was evaporated and redissolved around 5 times to remove excess of $I_{2}$ (azeotrope mixture with chloroform) until the solution become as slight pink as possible. A final flash filtering washing with cold ether yields a white solid $(7.3 \mathrm{~g}, 15.0 \mathrm{mmol})$ as final product $(84 \%)$. ${ }^{1} \mathbf{H}$ NMR $\left(300 \mathrm{MHz}, \mathrm{CDCl}_{3}\right): \delta=8.05$ (s, $\left.2 \mathrm{H}\right) .{ }^{13} \mathrm{C} \mathrm{NMR}\left(75 \mathrm{MHz}, \mathrm{CDCl}_{3}\right): \delta=142.68,125.50$, 106.97. EI HRMS: $\mathrm{m} / \mathrm{z}[\mathrm{M}+\mathrm{H}]^{+}$; for $\mathrm{C}_{6} \mathrm{H}_{3} \mathrm{Br}_{2} \mathrm{l}_{2}$ calc: 487.6592, 485.6613; found: 487.6595, 485.6617 .

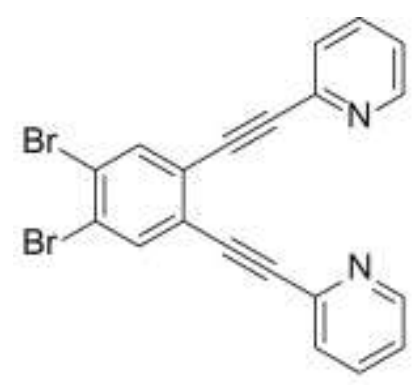

1,2-bis(ethyne-2,2"-dipyridine)-4,5-dibromobenzene, 2: 1 (2 g, $4.10 \mathrm{mmol}), \mathrm{PdCl}_{2}\left(\mathrm{PPh}_{3}\right)_{2}$ $(143.5 \mathrm{mg}, 0.21 \mathrm{mmol})$ and $\mathrm{Cul}(78 \mathrm{mg}, 0.41 \mathrm{mmol})$ were evacuated under protected athmosphere to add dry THF $(25 \mathrm{~mL})$ and degass it $(3 \mathrm{x})$. After this, it was added 2-ethynylpyridine $(0.89 \mathrm{mg}, 8.61 \mathrm{mmol})$ and $\mathrm{Et}_{3} \mathrm{~N}(1.25 \mathrm{~mL}, 0.91 \mathrm{~g}, 8.96 \mathrm{mmol})$ under protected atmosphere. The reaction stirred at room temperature overnight. The raw material was concentrated in vacuum and the crude product was purified by column chromatography $\left(\mathrm{CHCl}_{3} / \mathrm{MeOH}\right.$ 50:1 and a second column 100:1 $\mathrm{CHCl}_{3} / \mathrm{MeOH}$ ) to yield (84\%) a white solid (1.5 g, $3.4 \mathrm{mmol}) .{ }^{1} \mathrm{H}$ NMR $\left(300 \mathrm{MHz}, \mathrm{CDCl}_{3}\right): \delta=8.64(\mathrm{dt}, 2.4, J=1.2 \mathrm{~Hz}, 2 \mathrm{H}), 7.87(\mathrm{~s}, 2 \mathrm{H}), 7.67(\mathrm{~m}$, $4 \mathrm{H}), 7.23$ (m, $2 \mathrm{H}) .{ }^{13} \mathrm{C}$ NMR $\left(75 \mathrm{MHz}, \mathrm{CDCl}_{3}\right): \delta=147.34,140.02,139.60,137.15,129.31$, 126.77, 124.86, 124.36, 91.71, 90.05. ESI HRMS: $\mathrm{m} / \mathrm{z}[\mathrm{M}+\mathrm{H}]^{+}$; for $\mathrm{C}_{20} \mathrm{H}_{11} \mathrm{Br}_{2} \mathrm{~N}_{2}$ calc: 436.9283, 438.9264, 440.9243; found: 436.9269, 438.9260, 440.9229. 


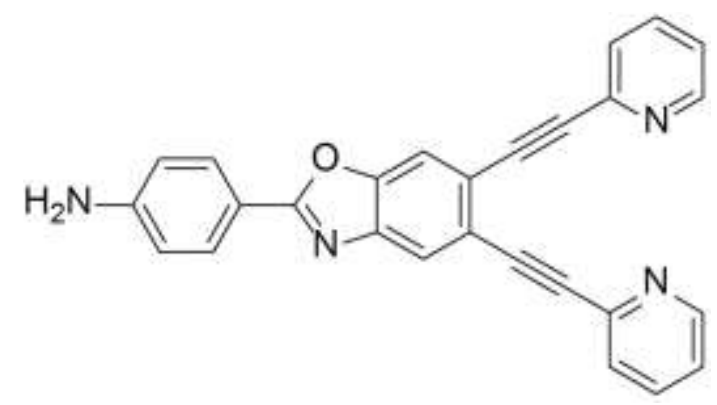

4-aniline-5,6-bis(ethyne-2,2"-dipyridine)-benzo-oxazol ${ }^{[96]}, 3: \mathrm{K}_{2} \mathrm{CO}_{3}(350 \mathrm{mg}, 2.53 \mathrm{mmol}), 2$ (200 mg, $0.46 \mathrm{mmol})$, Cul $(36.5 \mathrm{mg}, 0.19 \mathrm{mmol}$ ) and 4-aminobenzamide (203 mg, $1.49 \mathrm{mmol}$ ) were added to a flask and evacuated before addition of dry toluene $(9 \mathrm{~mL})$ and the mixture was degassed (3x). Under protected atmosphere it was added dmeda (30 $\mu \mathrm{L})$ (24.6 mg, $0.28 \mathrm{mmol}$ ) and the mixture was left to stirr at $120^{\circ} \mathrm{C}$ overnight. The crude material was concentrated in vacuum and purified by column chromatography $\mathrm{CHCl}_{3} / \mathrm{MeOH} 100: 1$ (twice) to yield $26 \%$ of a yellow solid (49 mg, $0.12 \mathrm{mmol}$ ). ${ }^{1} \mathrm{H} \mathrm{NMR}\left(300 \mathrm{MHz}, \mathrm{CDCl}_{3}\right): \delta=8.58(\mathrm{~d}, 6.6 \mathrm{~Hz}$, $2 \mathrm{H}, \mathrm{Ha}$ ), 7.97 (d, $8.7 \mathrm{~Hz}, 2 \mathrm{H}, \mathrm{Hg}), 7.87$ (s, $1 \mathrm{H}, \mathrm{He}$ ), 7.72 (s, $1 \mathrm{H} \mathrm{Hf}), 7.58$ (m, $4 \mathrm{H}, \mathrm{Hc}, \mathrm{Hd}), 7.17$ $(\mathrm{m}, 2 \mathrm{H}, \mathrm{Hb}), 6.68$ (d, 8.7 Hz, $2 \mathrm{H}, \mathrm{Hh}) .{ }^{13} \mathrm{C}$ NMR (75 MHz, d7-DMF): $\delta=166.73,151.71,143.21$, $138.08,133.21,132.98,132.72,131.71,130.37,129.70,127.75,125.52,122.48,98.24,86.77$. ESI HRMS: $\mathrm{m} / \mathrm{z}[\mathrm{M}+\mathrm{H}]^{+}$; for $\mathrm{C}_{27} \mathrm{H}_{17} \mathrm{~N}_{4} \mathrm{O}$ calc: 412.1397, 414.1430, 415.1464; found: 413.1391, 414.1407, 415.1437.

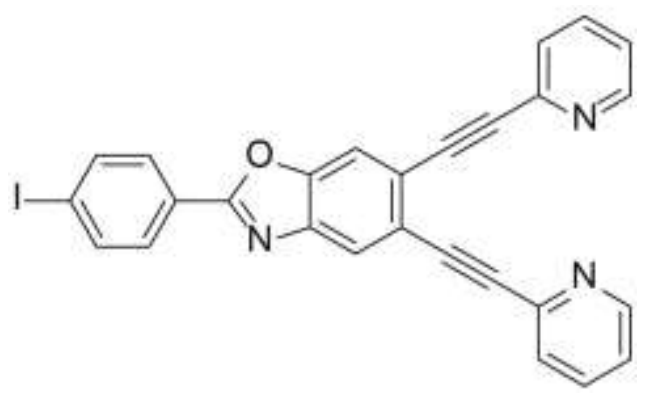

4-iodo-5,6-bis(ethyne-2,2"-dipyridine)-benzo-oxazol, 4: 3 (200 mg, $0.50 \mathrm{mmol}$ ) was added to a flask and evacuated previous to the addition of diiodo-methane $(2 \mathrm{~mL})$ and degassed before addition of iso-amyl-nitrite ( $333 \mu \mathrm{L}, 291 \mathrm{mg}, 2.48 \mathrm{mmol}$ ) under $\mathrm{N}_{2}$ atmosphere and a quickly degassing. The reaction was left to stirr for $48 \mathrm{~h}$ at $40^{\circ} \mathrm{C}$. The solvent was completelly removed in vacuum and the crude material was purified by column chromatography $\left(\mathrm{CHCl}_{3} / \mathrm{MeOH}\right)$ 100:1 yielding $20 \%$ of an orange solid material as final product $(50.7 \mathrm{mg}, 97 \mu \mathrm{mol}) .{ }^{1} \mathrm{H}$ NMR (300 MHz, CDCl $)_{3}$ ): $\delta=8.58(\mathrm{~d}, 4.5 \mathrm{~Hz}, 2 \mathrm{H}, \mathrm{Ha}), 7.93(\mathrm{~s}, 1 \mathrm{H}, \mathrm{He}), 7.88(\mathrm{~d}, 8.7 \mathrm{~Hz}, 2 \mathrm{H}, \mathrm{Hg}$ ), 7.80 (d, $8.7 \mathrm{~Hz}, 2 \mathrm{H}, \mathrm{Hh}), 7.76$ (s, $1 \mathrm{H}, \mathrm{Hf}), 7.59$ (m, $4 \mathrm{H}, \mathrm{Hc}, \mathrm{Hd}), 7.18$ (m, $2 \mathrm{H}, \mathrm{Hb})$.$) ). { }^{13} \mathrm{C}$ NMR (75 $\left.\mathrm{MHz}, \mathrm{CDCl}_{3}\right): \delta=165.69,150.49,142.65,136.45,132.50,131.84,130.90,130.70,128.37$, 
127.93, 127.40, 123.90, 122.21, 97.56, 86.17. ESI HRMS: $\mathrm{m} / \mathrm{z}[\mathrm{M}+\mathrm{H}]^{+}$; for $\mathrm{C}_{27} \mathrm{H}_{15} \mathrm{~N}_{3} \mathrm{Ol}$ calc: 524.0254, 525.0287, 526.0321; found: 524.0240, 525.0261, 526.0283.

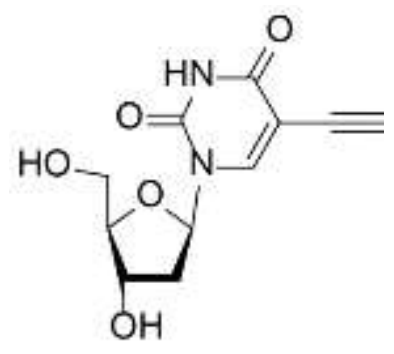

5-(eth-1-ynyl)-2 '-deoxyuridine, 5: 2'-iodo-deoxyuridine $(200 \mathrm{mg}, 0.56 \mathrm{mmol})$, Cul $(10.7 \mathrm{mg}$, $0.06 \mathrm{mmol})$ and $\mathrm{PdCl}_{2}\left(\mathrm{PPh}_{3}\right)_{2}(20 \mathrm{mg}, 0.03 \mathrm{mmol})$ were added to a flask and evacuated previous the addition ofdry THF $(5 \mathrm{~mL})$ and degassed (3x). After it, TMS-acetylene $(66.5 \mathrm{mg}$, $0.68 \mathrm{mmol})$ and $\mathrm{Et}_{3} \mathrm{~N}(0.5 \mathrm{~mL}, 36.3 \mathrm{mg}, 3.58 \mathrm{mmol})$ were added under protected atmosphere. The reaction was left to stir at room temperature for $4-5 \mathrm{~h}$ with the progress of the reaction been followed by TLC. The crude material was concentrated washed with chloroform through celite column and the solution concentrated. The crude material was (50 mg, $0.15 \mathrm{mmol}$ ) added to a Schlenk finger and evacuated before addition of dry $\mathrm{MeOH}$ and degassed under protected atmosphere. A solution of NaOMe $100 \mathrm{mM} \mathrm{(11} \mathrm{mL,} 60 \mathrm{mg}, 1.11 \mathrm{mmol}$ ) previously evacuated and degassed sepparatelly was added to the flask with the raw material through a syring and under protected $\mathrm{N}_{2}$. The reaction was left to stirr for $2 \mathrm{~h}$ at $r$.t. following the addition of DOWEX until reach a soution with $\mathrm{pH} \sim 5-6$. The solution was filtered and washed with $\mathrm{CHCl}_{3}$ before be concentrated and purified by column chromatography $\left(\mathrm{CHCl}_{3} / \mathrm{MeOH} 15: 1\right)$ yielding $13 \%$ of a white solid $\left(5 \mathrm{mg}, 0.20 \mathrm{mmol}\right.$ ) as final product. ${ }^{1} \mathrm{H}$ NMR $\left(300 \mathrm{MHz}, d^{6}-\mathrm{DMSO}\right): \delta=$ $11.63(\mathrm{~s}, 1 \mathrm{H}, \mathrm{N}-3$ ), 8.30 (s, $1 \mathrm{H}, \mathrm{H}-6), 6.09$ (dd, 6.6, $6.3 \mathrm{~Hz}, 1 \mathrm{H}, \mathrm{H}-1^{\prime}$ ), 5.24 (d, $4.5 \mathrm{~Hz}, 1 \mathrm{H}, \mathrm{OH}-$ 3'), $5.12\left(\mathrm{t}, 5.1 \mathrm{~Hz}, 1 \mathrm{H}, \mathrm{OH}-5^{\prime}\right), 4.21\left(\mathrm{~m}, 1 \mathrm{H}, \mathrm{H}-3^{\prime}\right), 4.10$ (s, $1 \mathrm{H}, \mathrm{C} \equiv \mathrm{CH}$ ), 3.79 (q, 3.6, 3.0. 3.6 Hz, $\left.1 \mathrm{H}, \mathrm{H}-4^{\prime}\right), 3.57$ (m, $\left.2 \mathrm{H}, \mathrm{H}-5^{\prime}\right), 2.12$ (dd, 6.6, $\left.4.8 \mathrm{~Hz}, 2 \mathrm{H}, \mathrm{H}-2^{\prime}\right) .{ }^{13} \mathrm{C} \mathrm{NMR}$ (75 MHz, d6-DMSO): $\delta=$ $161.72,149.47,144.56,97.61,87.64,84.87,83.60,76.46,70.03,60.88$. El HRMS: $\mathrm{m} / \mathrm{z}[\mathrm{M}+\mathrm{H}]^{+}$; for $\mathrm{C}_{11} \mathrm{H}_{13} \mathrm{~N}_{2} \mathrm{O}_{5}$ calc: 253.0780 , found: 253.0630 .

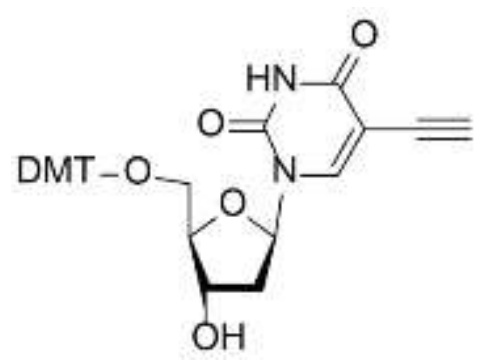


2'-deoxy-5'- 0-(4,4' - dimethoxytrityl)-5-(eth-1-ynyl)uridine 6: 5 (20 mg, $0.08 \mathrm{mmol}, 1$ eq) was added to a flask with dry pyridine $(1.5 \mathrm{~mL})$ and some molsieves and evacuated and degassed under $\mathrm{N}_{2}$ atmosphere. The reaction was left stirring for $1.5 \mathrm{~h}$ to add DMT-Cl $(32 \mathrm{mg}$, $0.09 \mathrm{mmol}$ ) and DMAP $(0.04 \mathrm{mg}, 0.03 \mathrm{nmol})$ under protecting athmosphere. The reaction was left stirring at room temperature overnight. The solution was concentrated and the crude material was purified by column chromatography $100: 1 \mathrm{CHCl}_{3} / \mathrm{MeOH}+0.1 \%$ py. A colorless oil was collected as final product and submitted to vacuum until evaporate the excess of pyridine and produce a yellowish solid foam $(28.5 \mathrm{mg}, 0.88 \mu \mathrm{mol}$, yield: $65 \%) .{ }^{1} \mathrm{H}$ NMR (300 MHz, $d^{6}-$ DMSO): $\delta=11.68$ (s, $1 \mathrm{H}, \mathrm{N}-3), 7.94(\mathrm{~s}, 1 \mathrm{H}, \mathrm{H}-6), 7.33$ (m, $5 \mathrm{H}, \mathrm{H} 3, \mathrm{H} 4, \mathrm{H} 5), 7.41(\mathrm{~m}, 4 \mathrm{H}, \mathrm{H} 2)$, 6.90 (d, 8.7 Hz, 4 H, H1), 6.09 (dd, 6.6, $6.3 \mathrm{~Hz}, 1 \mathrm{H}, \mathrm{H}-1^{\prime}$ ), 5.35 (d, $\left.4.5 \mathrm{~Hz}, 1 \mathrm{H}, \mathrm{OH}-3^{\prime}\right), 4.25$ (m, $\left.1 \mathrm{H}, \mathrm{H}-3^{\prime}\right), 3.96(\mathrm{~s}, 1 \mathrm{H}, \mathrm{C} \equiv \mathrm{CH}), 3.96\left(\mathrm{~m}, 1 \mathrm{H}, \mathrm{H}-4^{\prime}\right), 3.15\left(\mathrm{~m}, 2 \mathrm{H}, \mathrm{H}-5^{\prime}\right.$, partially overlapped on DMSO peak), 2.67 (m, $\left.2 \mathrm{H}, \mathrm{H}-2^{\prime}\right) .{ }^{13} \mathrm{C}$ NMR (75 MHz, d6-DMSO): $\delta=161.72,149.47,144.56$, 97.61, 87.64, 84.87, 83.60, 76.46, 70.03, 60.88. ESI MS: $\mathrm{m} / \mathrm{z}[\mathrm{M}+\mathrm{H}]^{+}$; for $\mathrm{C}_{32} \mathrm{H}_{31} \mathrm{~N}_{2} \mathrm{O}_{7}$ calc: 555.21, found: 555.20 .

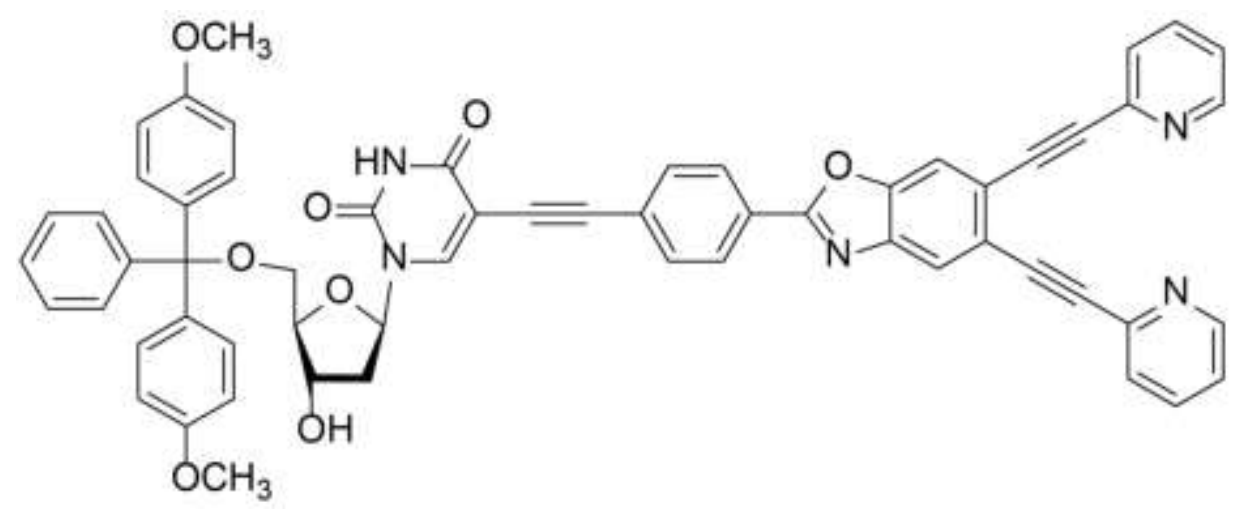

2'-deoxy-5' - 0-(4,4' - dimethoxytrityl)-5-(eth-1-ynyl)-5,6-bis(ethyne-2,2"-dipyridine)-benzooxazol-uridine, 7: 4 (90 mg, $0.17 \mathrm{mmol}$ ), 6 (124 mg, $0.22 \mathrm{mmol}), \mathrm{PdCl}_{2}\left(\mathrm{PPh}_{3}\right)_{2}(6 \mathrm{mg}, 8.57 \mu \mathrm{mol})$ and $\mathrm{Cul}(1.6 \mathrm{mg}, 8.57 \mu \mathrm{mol})$ were added in a flask and evacuated before addition of dry DMF $(3.5 \mathrm{~mL})$ under protected athmosphere and be degassed. The reaction was stirred at $70^{\circ} \mathrm{C}$ for 1h before addition of dropwising $\mathrm{Et}_{3} \mathrm{~N}(0.5 \mathrm{~mL}, 0.36 \mathrm{~g}, 3.58 \mathrm{mmol})$. The reaction was left to stirr overnight at $70^{\circ} \mathrm{C}$. The crude material was concentrated in vacuum and submitted to column chromatography: $1^{\text {st }}$ column $100: 1 \mathrm{CHCl}_{3} / \mathrm{MeOH}$ and $2^{\text {nd }}$ column $50: 1 \mathrm{CHCl}_{3} / \mathrm{MeOH}$, giving a yellow solid $\left(106 \mathrm{mg}, 0.11 \mathrm{mmol}\right.$ ) with the yield of $65 \%$ as final product. ${ }^{1} \mathbf{H}$ NMR (300 MHz, d $d^{6}$-DMSO): $\delta=11.82(\mathrm{~s}, 1 \mathrm{H}, \mathrm{N}-3), 8.69(\mathrm{~m}, 2 \mathrm{H}, \mathrm{Ha}), 8.25(\mathrm{~s}, 1 \mathrm{H}, \mathrm{He}), 8.22(\mathrm{~s}, 1 \mathrm{H}$, Hf), $8.21(\mathrm{~s}, 1 \mathrm{H}, \mathrm{H}-6), 8.14$ (d, 8.4 Hz, $2 \mathrm{H}, \mathrm{Hg}$ ), 7.91 (m, $4 \mathrm{H}, \mathrm{Hc}, \mathrm{Hd}), 7.47$ (d, $7.5 \mathrm{~Hz}, 4 \mathrm{H}, \mathrm{Hb})$, 7.35 (m, 5 H, H3, H4, H5), 7.27 (d, 8.4 Hz, 2 H, Hh), 6.89 (dd, 1.8, 9.0, $10.5 \mathrm{~Hz}, 4 \mathrm{H}, \mathrm{H} 2$ ), 6.19 (dd, 6.3, 13.2 Hz, $\left.1 \mathrm{H}, \mathrm{H}-1^{\prime}\right), 5.38\left(\mathrm{~d}, 4.5 \mathrm{~Hz}, 1 \mathrm{H}, \mathrm{OH}-3^{\prime}\right), 4.37\left(\mathrm{~m}, 1 \mathrm{H}, \mathrm{H}-3^{\prime}\right), 4.01\left(\mathrm{~m}, 1 \mathrm{H}, \mathrm{H}-4^{\prime}\right)$, 
$3.75\left(m, 2 \mathrm{H}, \mathrm{H}-5^{\prime}\right), 2.66\left(\mathrm{~m}, 2 \mathrm{H}, \mathrm{H}-2^{\prime}\right)$. The compound was not soluble enough to obtain ${ }^{13} \mathrm{C}$ NMR. ESI HRMS: $\mathrm{m} / \mathrm{z}[\mathrm{M}+\mathrm{H}]^{+}$; for $\mathrm{C}_{59} \mathrm{H}_{44} \mathrm{~N}_{5} \mathrm{O}_{8}$ calc: 950.3184, 951.3217, 952.3251, 953.3285; found: $950.3172,951.3206,952.3239,953.3287$.

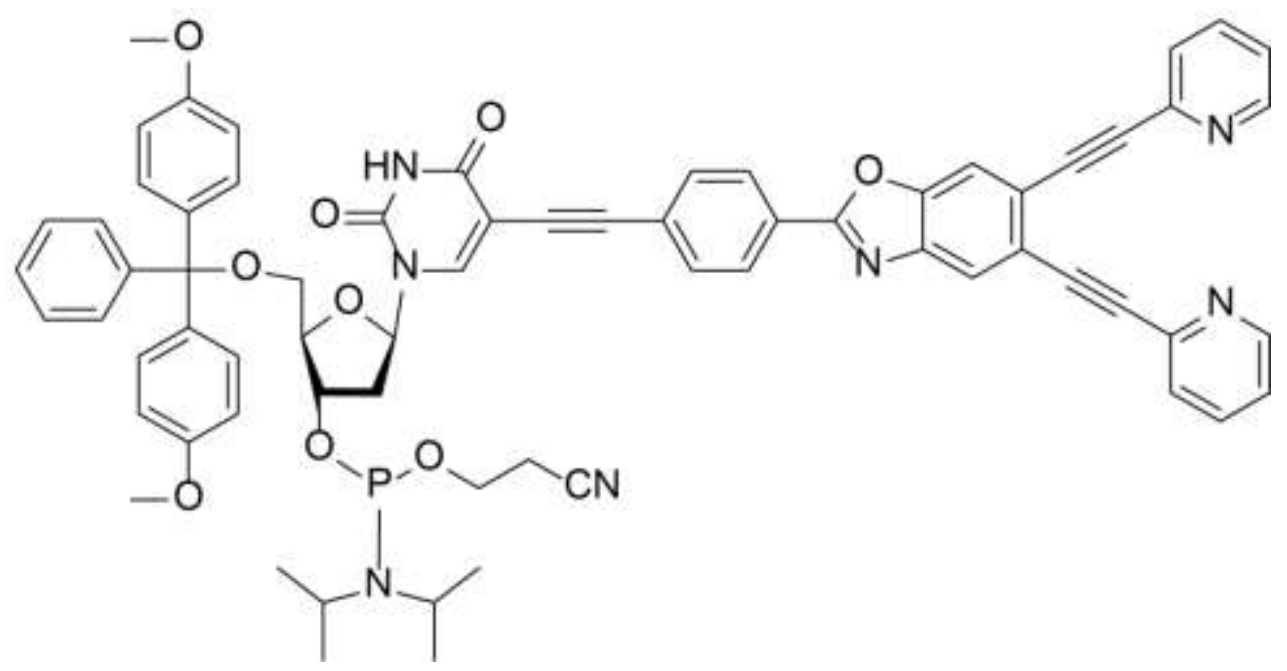

\section{2'-deoxy-5'- 0-(4,4' - dimethoxytrityl)-5-(eth-1-ynyl)-5,6-bis(ethyne-2,2"-dipyridine)-benzo-}

oxazol-uridine-3'- phosphoramidite, 8: 7 (160 mg, $0.17 \mathrm{mmol})$ was evacuated in a flask with some molsives $3 \AA$ and dry THF been co-evaporated twice and degassed. After the reaction stirr for $0.5 \mathrm{~h}$, it was added (iPr) ${ }_{2} \mathrm{NEt}(109 \mathrm{mg}, 0.84 \mathrm{mmol})$ and quickly degassed again before the addition of 2-cyanoethyl $\mathrm{N}, \mathrm{N}$-diisopropyl, CEP-Cl, $(120 \mathrm{mg}, 0.51 \mathrm{mmol})$. The reaction was left to stir for $1 \mathrm{~h}$ and the progress of the reaction was followed by TLC. The solvent was reduced to about $0.5 \mathrm{~mL}$ in vacuum and cold dry hexane $(\sim 1 \mathrm{~mL})$ was added. The final product precipitated in the wall of the glas and producing a milky solution containing salts and other impurities which were removed through a needle under $\mathrm{N}_{2}$. After this, the material was evaporated by vacuum to provide a yellowish solid product $(90 \mathrm{mg}, 0.08 \mathrm{mmol})$. The estimated yield was around $46 \%$. The product it is not stable at room temperature and without be kept under protected atmosphere, been submitted right after its synthesis to dilution in dry dichloro-ethane under protected atmosphere and applied for solid phase DNA synthesis. Due to such conditions restricted by time and sensitivity, it was not possible to obtain a spectrum of ${ }^{1} \mathbf{H}$ NMR for the final product. ${ }^{31} \mathbf{P}$ NMR $\left(40 \mathrm{MHz} \mathrm{CDCl}_{3}\right): \delta=149.07,148.61$.

Unmodified oligonucleotides: Unmodified oligonucleotides $s 4^{*}$ and $s 5^{*}$ were purchased by Biomers.net $\mathrm{GmbH}$ in aqueous solution with concentration $200.0 \mathrm{pmol} / \mu \mathrm{l}$ and purified by HPLC. Sequences for both strands, $\mathbf{s} \mathbf{4}^{*}$ and $\mathbf{s} \mathbf{5}^{*}$, are respective to the sequence for modified strands, containing $d T$ instead of $X$ for $s 4^{*}$ and $d A$ for $s 5^{*}$. 


\section{Crystal structure for trans-chelating nucleoside, 7}

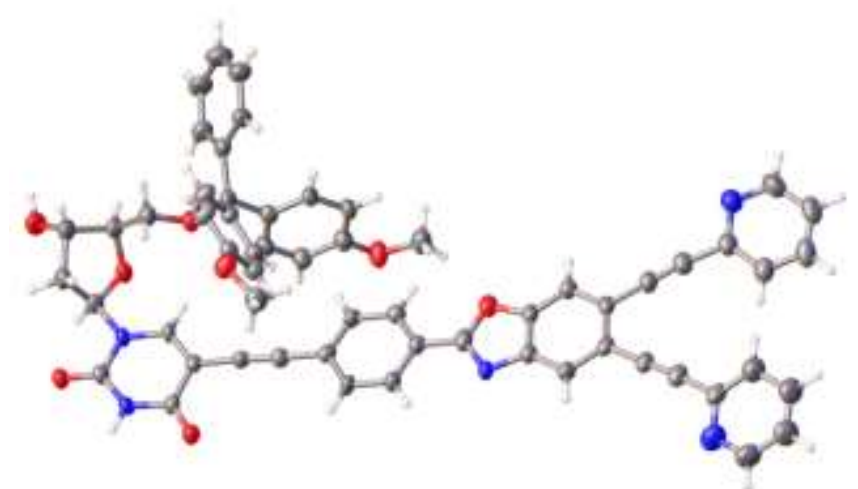

Figure 96. Crystal structure for the trans-chelating nucleoside by Dr. Mark Light in Southampton University, UK.

Single crystals of $\mathbf{7}$ were obtained by recrystallisation in $\mathrm{CHCl}_{3} / \mathrm{MeOH}+0.1 \%$ py. A crystal $\left(0.09 \times 0.04 \times 0.01 \mathrm{~mm}^{3}\right)$ was selected with a MITIGEN holder in perflourotether oil on a Rigaku AFC12 FRE+ diffractometer. The crystal was kept at $100(2) \mathrm{K}$ during data collection. The structure was solved with the olex2.solve structure solution program, using the Charge Flipping solution method. The model was refined with the ShelXL ${ }^{[97]}$ refinement package using Least Squares minimization. Crystal Data. $\mathrm{C}_{60.5} \mathrm{H}_{54} \mathrm{~N}_{5} \mathrm{O}_{12}, \mathrm{M}=1043.08$, triclinic, P1 (No. 1, $a=13.1722 \AA, b=13.9088 \AA, c=14.5058 \AA, a=93.339^{\circ}, b=93.854^{\circ}, g=93.844^{\circ}, V=2640.4(6)$ $\AA^{3}, T=100(2) K, Z=2, m\left(M o K_{a}\right)=0.092,28745$ reflections measured, 14185 unique $\left(R_{\text {int }}=\right.$ 0.0481 ). The final $w R_{2}$ was 0.2727 (all data) and $R_{1}$ was $0.0898(I>2(I))$. 
Table 9. Crystallographic data for compound 7

\begin{tabular}{|c|c|c|c|}
\hline Compound & 7 & & \\
\hline CCDC & 1478186 & & \\
\hline Formula & $\mathrm{C}_{60.5} \mathrm{H}_{54} \mathrm{~N}_{5} \mathrm{O}_{12}$ & $V / \AA^{3}$ & $2640.4(6)$ \\
\hline $\mathrm{C}_{59} \mathrm{H}_{43} \mathrm{~N}_{5} \mathrm{O}_{8}$ & $1.5\left(\mathrm{CH}_{4} \mathrm{O}\right), 2.5\left(\mathrm{H}_{2} \mathrm{O}\right)$ & Z & 2 \\
\hline D calc. $/ \mathrm{g} \mathrm{cm}^{-3}$ & 1.312 & Theta $\min /^{\circ}$ & 2.942 \\
\hline $\mathrm{m} / \mathrm{mm}^{-1}$ & 0.092 & Theta $\max /^{\circ}$ & 25.028 \\
\hline Formula Weight & 1043.08 & Measured Refl. & 28745 \\
\hline Colour & clear orange & Independent Refl. & 14185 \\
\hline Shape & plate & Reflections Used & 9666 \\
\hline Size $/ \mathrm{mm}^{3}$ & $0.09 \times 0.04 \times 0.01$ & $\mathrm{R}$ (int) & 0.0481 \\
\hline $\mathrm{T} / \mathrm{K}$ & $100(2)$ & Parameters & 1386 \\
\hline Crystal System & triclinic & Restraints & 1230 \\
\hline Space Group & P1 & Largest Peak & 1.115 \\
\hline $\mathrm{a} / \AA$ & $13.1722(17)$ & Deepest Hole & -0.474 \\
\hline $\mathrm{b} / \AA$ & $13.9088(18)$ & GooF & 1.025 \\
\hline$c / \AA$ & $14.5058(18)$ & $w R_{2}$ (all data) & 0.2727 \\
\hline$a /^{\circ}$ & $93.339(3)$ & $w \mathrm{R}_{2}$ & 0.2359 \\
\hline $\mathrm{b} /{ }^{\circ}$ & $93.854(3)$ & $\mathrm{R}_{1}$ (all data) & 0.1295 \\
\hline $\mathrm{g} /$ & $93.844(3)$ & $\mathrm{R}_{1}$ & 0.0898 \\
\hline
\end{tabular}

\subsection{DNA Syntheses}

Oligonucleotides were synthesized by solid phase synthesis with a Applied Biosystems Expedite (part 3) machine or a K\&A Synthesizer H-8 (part 2 and 3), both on a 1 umol scale. All the solvents were purchased from Sigma Aldrich, Carl Roth or Fisher Scientific under dry conditions and still extra dried over molecular sieves $3 \AA$ with $24 \mathrm{~h}$ previous to DNA synthesis. The solutions required for DNA synthesis were prepared according to tables 10 and 11 and the reagents purchased by Sigma Aldrich and Carl Roth. The protected phosphoramidites used for DNA synthesis were purchase by Sigma aldrich. Benzylthio-1 $H$-tetrazole (BTT) was synthesized following the literature. ${ }^{[98]}$ Controlled pore glass, CPG, solid supports (30 $\mu \mathrm{mol} / \mathrm{g} .1000 \AA$ ) were purchased in plastic columns from $K \& A$ Laborgeraete $G b R$. 
The oligonucleotides were synthesized as DMT-ON mode with extended coupling time (15 minutes) for the artificial phosphoramidites. The chosen sequences were based on literature. ${ }^{[99]} 5^{\prime}$-DMT protected nucleotide was deblocked with $3 \%$ trichloroacetic acid solution, TCA, in dichloro-methane. $0.3 \mathrm{M}$ benzylthio-1 $\mathrm{H}$-tetrazole (BTT) in dry acetonitrile was used as activator for the 3'-phosphoramidite. As Cap B for capping unreacted 5'-hydroxyls was used acetic anhydride in dry THF/pyridine and as Cap A was used methylimidazole in a mixture of dry THF/pyridine. The oxidation was performed with a solution of $0.02 \mathrm{M}$ lodine in $\mathrm{THF} /$ pyridine/water. Dry acetonitrile was used for the washing steps.

Table 10. Values for preparation of the phosphoramidites.

\begin{tabular}{c|c}
\hline $\begin{array}{c}\text { 0.1 M solutions } \\
\text { in dry MeCN }\end{array}$ & $\begin{array}{c}\text { Dry MeCN amount for } \\
\mathbf{0 . 5} \text { g phosphoramidites }\end{array}$ \\
\hline dA-CE & $5.8 \mathrm{~mL}$ \\
dC-CE & $6.0 \mathrm{~mL}$ \\
dG-CE & $6.0 \mathrm{~mL}$ \\
dT-CE & $6.7 \mathrm{~mL}$ \\
\hline
\end{tabular}

Table 11. Details about the solutions for SPS.

\begin{tabular}{|c|c|c|c|}
\hline DNA synthesis steps & reagent & Solution preparation & Solution stability \\
\hline Detritylation & $\begin{array}{c}3 \%(v / v) \text { trichloroacetic } \\
\text { acid, TCA }\end{array}$ & $\begin{array}{c}3 \mathrm{~g} \text { TCA in } 100 \mathrm{~mL} \\
\text { dry } \mathrm{CH}_{2} \mathrm{Cl}_{2}\end{array}$ & Long termn stable \\
\hline Activation & $\begin{array}{l}0.3 \mathrm{M} \text { Benzylthio-1H- } \\
\text { tetrazole, BTT }\end{array}$ & $\begin{array}{c}5,76 \mathrm{~g} \mathrm{BTT} / 100 \mathrm{~mL} \\
\text { dry MeCN }\end{array}$ & $\begin{array}{l}\text { Not long term } \\
\text { stable }\end{array}$ \\
\hline Capping A, Cap A & $\begin{array}{c}16 \%(v / v) \text { N-Methyl- } \\
\text { imidazole, Melm }\end{array}$ & $\begin{array}{l}16 \mathrm{~mL} \text { of Melm/ } \\
84 \mathrm{~mL} \text { dry THF }\end{array}$ & Long term stable \\
\hline Capping B, Cap B & $\begin{array}{c}10 \%(v / v) \text { Acetic } \\
\text { anhydride }\end{array}$ & 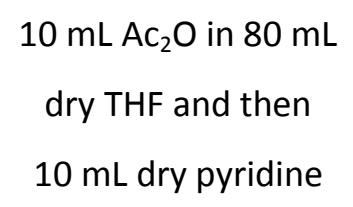 & $\begin{array}{l}\text { Not long term } \\
\text { stable }\end{array}$ \\
\hline Oxidizer & 0.02 M lodine & $\begin{array}{c}5.1 \mathrm{~g} 12 \mathrm{in} 20 \mathrm{~mL} \\
\text { pyridine, then } 70 \mathrm{~mL} \\
\text { THF and } 10 \mathrm{~mL} \text { water }\end{array}$ & Long term stable \\
\hline Washing & Dry Acetonitrile, $\mathrm{MeCN}$ & - & - \\
\hline
\end{tabular}




\subsection{Purification by HPLC, detritylation and desalting}

After the DNA synthesis the solid resin was removed from the plastic columns and the cleavage from the solid support of the oligonucleotides sequences synthesized and deprotection for phosphoramidites was done by a $3 \mathrm{M}$ ammonium hydroxide solution with methanol $(50: 50,1 \mathrm{~mL})$ left for 8 hours at $55-60^{\circ} \mathrm{C}$ in a thermomixer. The oligonucleotides were then filtrated and the supernatant was evaporated for ammonia evaporation and solution concentration by a vacuum centrifuge concentrator (S-Concentrator BA-VC-300H, H.Saur) before purification by RP-HPLC. Synthesized DNA sequences DMT-ON were purified using Agilent 1260 Infinity HPLC system equipped with a Macherey Nagel 250/10 Nucleodur 100-5 C18ec column or a Varian 920-LC system with a Polaris 3 150/4.6 mm C18-A column. The gradient used was $3 \mathrm{~mL} / \mathrm{min}$., $20-40^{\circ} \mathrm{C}$ with $85 \%$ of solvent $A$ (10 min.), $60 \%$ solvent $A(5 \mathrm{~min}$.), $100 \%$ solvent $A$ for the 10 last minutes. Solvent $A$ has as solution $0.1 \mathrm{~mol} \mathrm{~L}^{-1} \mathrm{TEAA}, \mathrm{pH} 7$ and solvent $B$ has as solution 70:30 MeCN/ TEAA $0.1 \mathrm{~mol} / \mathrm{L}, \mathrm{pH} 7$, acetonitrile purchased by VWR International or Sigma Aldrich. The samples were then concentrated after HPLC and detritylated/desalted with Glen-Pak ${ }^{\mathrm{TM}}$ cartridges by Glen Research based on provided instructions from the fabricant. The determination of the concentration for the DNA samples were done based on the absorbance at $260 \mathrm{~nm}$. The molar absorptivity of the artificial nucleosides were determined by UV/Vis dilution assay with details given in respective results parts. The extinction coefficients for the strands synthesized were determined using the base composition method and with the following equation $\varepsilon_{260}=((n A \times 15.4)+(n C \times 7.4)+(n G \times$ 11.5 $)+(n T \times 8.7)) \times 0.9 \times 1000$ L. $\mathrm{mol}^{-1} \cdot \mathrm{cm}^{-1}$ using values for each base according to the literature. $^{[100]}$

\subsection{ESI MS characterization for the oligonucleotides}

DNA samples under concentration of $20 \mu \mathrm{M}$ were prepared in aqueous solution (milli $Q$ water) with MeCN at ratio 50:50 and measured in a system Bruker ESI-TOF MaXis 10136 and also characterization of single strands with a Bruker microTOF mass spectrometer coupled to a Dionex UltiMate $3000 \mathrm{HPLC}$ system. Temperature source $150^{\circ} \mathrm{C}$ for characterization. For measurements to detect nanoconstructs based on ds-DNA duplex and their correspondent metallated nanoconstructs, temperature $100-150^{\circ} \mathrm{C}$, capillary of $3000 \mathrm{~V}$, nebulizer $0.3 \mathrm{psi}$, dry gas of $3.0 \mathrm{I} / \mathrm{min}$, collision cell energy of $8 \mathrm{eV}$ with collision RF of $1000.0 \mathrm{Vpp}$ and Pre plus storage of $10.0 \mu \mathrm{s}$. The sample conditions for DNA duplexes consist to an aqueous solution of $100 \mathrm{mM}$ ammonium acetate, $\mathrm{NH}_{4} \mathrm{CH}_{3} \mathrm{CO}_{2}, \mathrm{pH} 8.3$ and $\mathrm{MeCN}$ or MeOH (50:50). 


\subsection{Thermal analysis and UV/Vis spectroscopy}

The preparation of the ds-DNA samples used for all following spectroscopic measurements (UV/Vis, thermal analysis, CD, Fluorescence and FRET) were performed in aqueous solution in presence of buffer and salt indicated in the specific part of the results (with blank measurement of water, buffer and salt been subtracted from the spectrum measured). The protocoll for annealing the DNA duplexes are as follows: $95{ }^{\circ} \mathrm{C}\left(5 \mathrm{~min}\right.$.), $65{ }^{\circ} \mathrm{C}\left(20 \mathrm{~min}\right.$.), $50{ }^{\circ} \mathrm{C}$ (15 min.), $37{ }^{\circ} \mathrm{C}(20 \mathrm{~min}$.) and at r.t. (20 min.). The UV/Vis spectra were measured using a JASCO-UV/VIS-Spectrometer model V-650 with a peltier accessory ETCS-761, UV/Vis bandwidth $1.0 \mathrm{~nm}$ and change source at $350 \mathrm{~nm}$ with scan speed of $200 \mathrm{~nm} / \mathrm{min}$ at room temperature. The thermal analysis were measured using a JASCO-UV/VIS-Spectrometer also model V-650 with a Peltier control PAC-743, UV/Vis bandwidth $1.0 \mathrm{~nm}$, measurements at 260 $\mathrm{nm}$ and $500 \mathrm{~nm}$ and change of light source at $340 \mathrm{~nm}$, from $4^{\circ} \mathrm{C}$ to $85^{\circ} \mathrm{C}$ and a temperature gradient of $0.5^{\circ} \mathrm{C} / \mathrm{min}$ or $0.1^{\circ} \mathrm{C} / \mathrm{min}$ (to be specified on the specific results parts). To measure DNA samples 3-4 $\mu \mathrm{M}$ concentration were used quartz cuvettes with $l=10 \mathrm{~mm}$ (Hellma Analytics 114-QS). The samples before submitted to thermal analysis were sealed with an silicon oil layer and during the measurement dry air was pumped inside the measurement cell to avoid condensation of water. Melting curves were normalized based on $[0,1]$ limits and the melting points were determined by the first derivative method with a curve smoothing of 10 points (Savitzky-Golay). Details for each oligonucleotide preparation can be found in details on the respective result part.

\subsection{Fluorescence and FRET spectroscopy}

A Fluorolog ${ }^{\circledR}-3$ FluorEssence ${ }^{\mathrm{TM}}$ FL-1057 device was used to measured all the fluorescence spectra, using quartz cuvette with dimensions of $10 \times 4 \mathrm{~mm}$. The excitation performed vary according to the samples (to be specified according to the sample on results together with sample preparation details) from $330-360 \mathrm{~nm}$ and the emission range of $340-600 \mathrm{~nm}$ for all samples (slit width 2.0 and $5.0 \mathrm{~nm}$ according to the sample).

The blank measurement (water, buffer and electrolyte) was subtracted from all measurements taken. A second blank containing the counterstrand carrying the FRET pair, $\mathbf{s} \mathbf{2}^{\mathrm{D}}$, was also measured (UV/Vis and Fluorescence) as a control experiment and its emission spectra upon addition of $n$ eq. of $\mathrm{Cu}(\mathrm{II})$ were subtracted from the data obtained for the data of $\mathbf{s} 3 . \mathbf{s} \mathbf{2}^{\mathrm{D}}+\mathrm{n}$ eq. of $\mathrm{Cu}(\mathrm{II})$ (appendix Figure 101) in order to subtract any value correspondent to interactions between copper(II) and the FRET pair, remanining in the results only the information regarding 
the interactions through the weaking coupling established by the inter cross-link of two s3 strands connected by the complexation of the two trans-chelating motif and $\mathrm{Cu}(\mathrm{II})$.

\subsection{Circular Dichroism spectroscopy}

CD spectra were measured with a JASCO spectrometer, model J-1500, detectors PM-539 and FDT-538 with an accessory PTC-510. DNA sample concentration 3-4 $\mu \mathrm{M}, I=1 \mathrm{~cm}$, at room temperature and scanning speed $100 \mathrm{~nm} / \mathrm{min}$ with 3 accumulations each spectrum. The spectra had the background corrected (extracted the blank basen on aqueous buffer solution and electrolyte) and smoothed (FFT). Details for each oligonucleotide sample preparation can be found on details on respective result part.

\subsection{Polyacrylamide gel electrophoresis, PAGE}

Non-denaturing gel electrophoresis were performed with a CBS Dual-Vertical Gel DSG-200-2 system, by CBS Scientific, under constant voltage of 200 V, 20 mA. 8\% polyacrylamide gels with stacking gels around $4 \%$ were made with Tris-boron-magnesium, TBM, running buffer (890 mM tris- $\left.\mathrm{HCl}, 12 \mathrm{mM} \mathrm{Mg(OAc})_{2}, \mathrm{pH} 8.3\right)$. The DNA samples with concentration vary between 250-500 ng (described in each respective case) containing 0.2 - $0.5 \mu \mathrm{mol} \mathrm{Mg}(\mathrm{OAc})_{2}$. The duplexes were annealed as follows: $95^{\circ} \mathrm{C}(5 \mathrm{~min}),. 65^{\circ} \mathrm{C}(20 \mathrm{~min}),. 50^{\circ} \mathrm{C}(15 \mathrm{~min}),. 37^{\circ} \mathrm{C}(20$ min.) and at r. t. (20 min.). The gels previous to be loaded with samples were conditioned with running buffer for 10-15 minutes. The time for the gels run vary between 2-5 $\mathrm{h}$ and was submitted at $5^{\circ} \mathrm{C}$.

Table 12. Amounts of reagents for the GEL preparation. Values for $10 \mathrm{~mL}$ of solution.

\begin{tabular}{c|c|c}
\hline \% of the gel & Running gel (8\%) & Stacking gel (4\%) \\
\hline Acrylamide & $2.66 \mathrm{~mL}$ & $1.34 \mathrm{~mL}$ \\
10\% APS & $100 \mu \mathrm{L}$ & $100 \mu \mathrm{L}$ \\
TEMED & $10 \mu \mathrm{L}$ & $10 \mu \mathrm{L}$ \\
$\mathrm{H}_{2} \mathrm{O}$ & $7.24 \mathrm{~mL}$ & $8.56 \mathrm{~mL}$ \\
\hline
\end{tabular}

As marker it was used DNA ladder O'Range Ruler 10-150 bp from Thermo Scientific or $10 \mathrm{bp}$ DNA ladder by Invitrogen. As stain reagent it was used SYBR ${ }^{\circledR}$ Gold Nucleic Acid Stain by Invitrogen $^{\mathrm{TM}}$. The pictures taken were irradiated with UV light at $305 \mathrm{~nm}$ excitation with a UV 
transilluminator and photos were done before stain and after stain with DIANA gel imaging device, with Raytest for non-radioactive application and a cooled CCD camera with a light-tight setup on a UV-light table and a normal domestic camera. Brightness and contrast of the photos were adjusted to stay uniform.

\section{Appendix}

\subsection{Additional Figures}

\section{Part 1)}

The geometry optimization of the self-assembled rings RING1 (a) and RING3 (b) viewed by front and side (Gaussian '09, semiempiric PM6 level of theory). For RING3 the $C_{2}$ geometry of the dibenzosuberone ligand was sustained according to its X-ray structure ${ }^{[83]}$. It was found that PM6 shortcomes in correctly describing the conformation of the seven-membered ring ${ }^{[101]}$, necessary to fix the dihedral angles of the ethylene bridge with the annelated benzene.
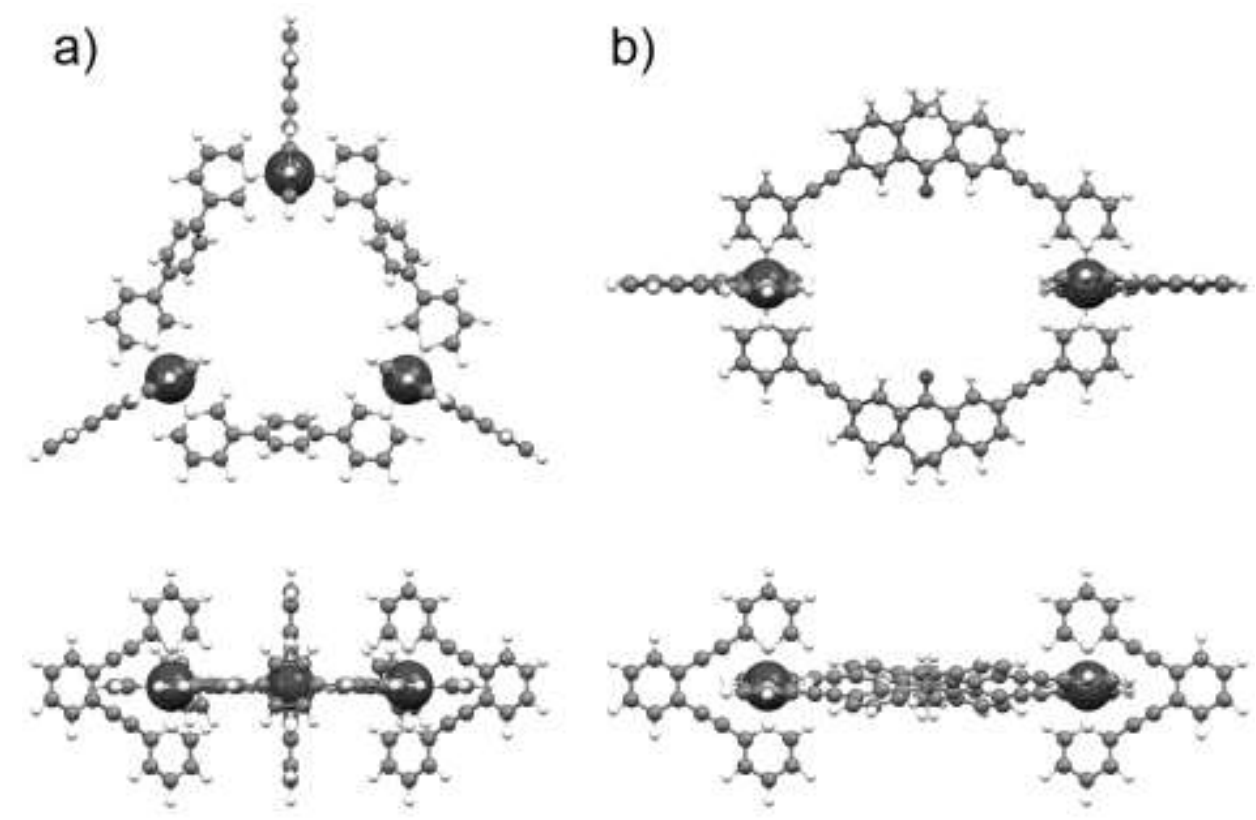

Figure 97. Appendix figure showing the modeling (Gaussian 09) for a) 3D membered RING1 with $\left\{\mathrm{Pd}_{3}(\mathbf{L 2})_{3}\right\}$ fragments and b) 2D membered RING3 with $\left\{\mathrm{Pd}_{2}\left(\mathbf{L}_{2}\right)_{2}\right\}$ fragments. 


\section{Part 2)}
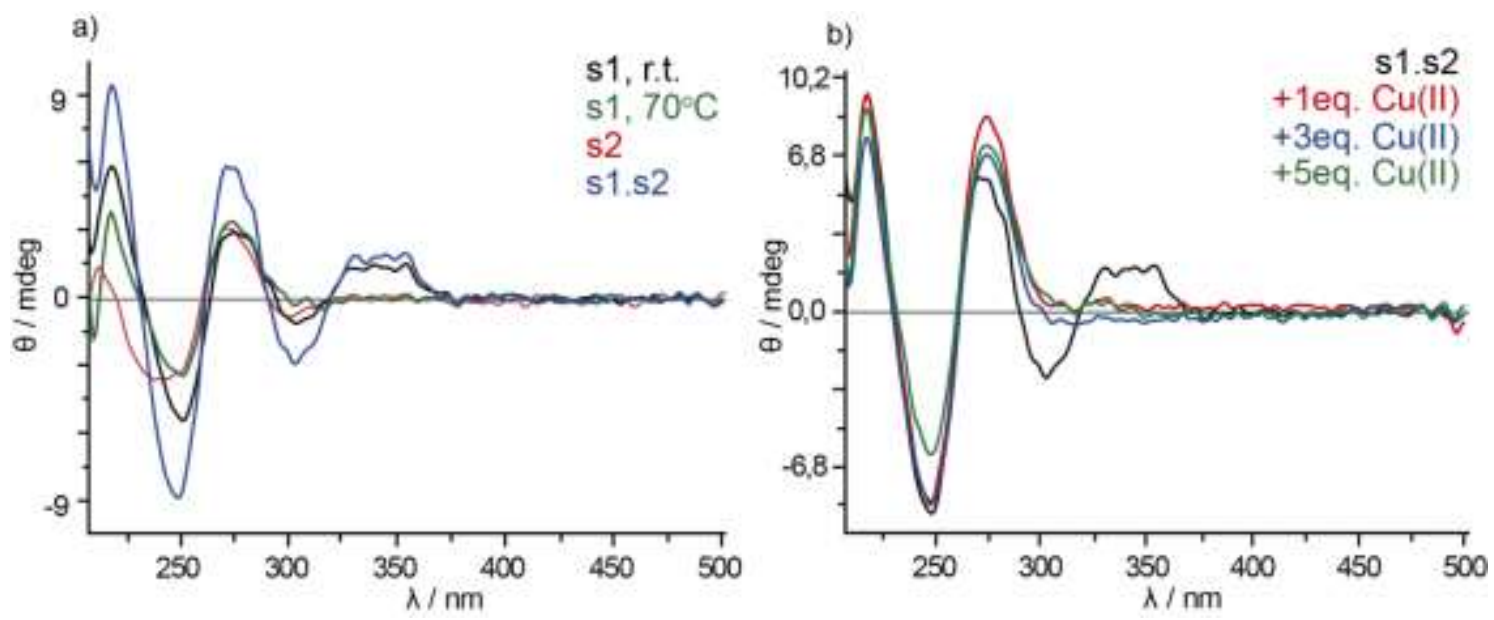

Figure 98. Appendix figure of $\mathrm{CD}$ spectra for $3 \mu \mathrm{M}$ DNA, $100 \mathrm{mM} \mathrm{NaClO}_{4}, 10 \mathrm{mM}$ cacodylate buffer, a) s1, s2, s1.s2, b) s1.s2 + neq. Cu(II).

\section{Part 3)}
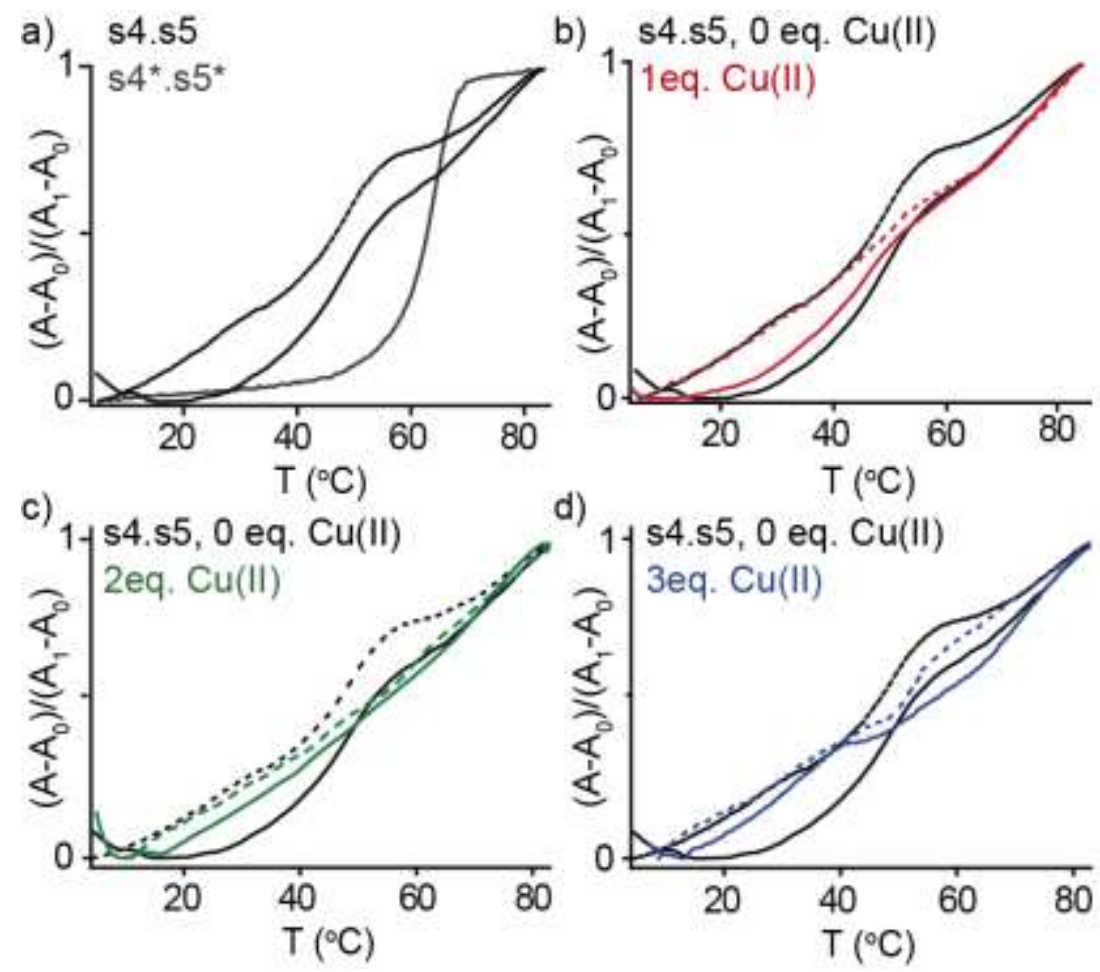

Figure 99. Appendix fugure for thermal analysis for duplex $3 \mu \mathrm{M} \mathbf{s 3 . s 2}$, $100 \mathrm{mM} \mathrm{NaCl}, 10 \mathrm{mM}$ cacodylate buffer $+\mathrm{n}$ eq. of $\mathrm{Cu}(\mathrm{II})$. Temperature rate $0.1^{\circ} \mathrm{C} / \mathrm{min}$. 

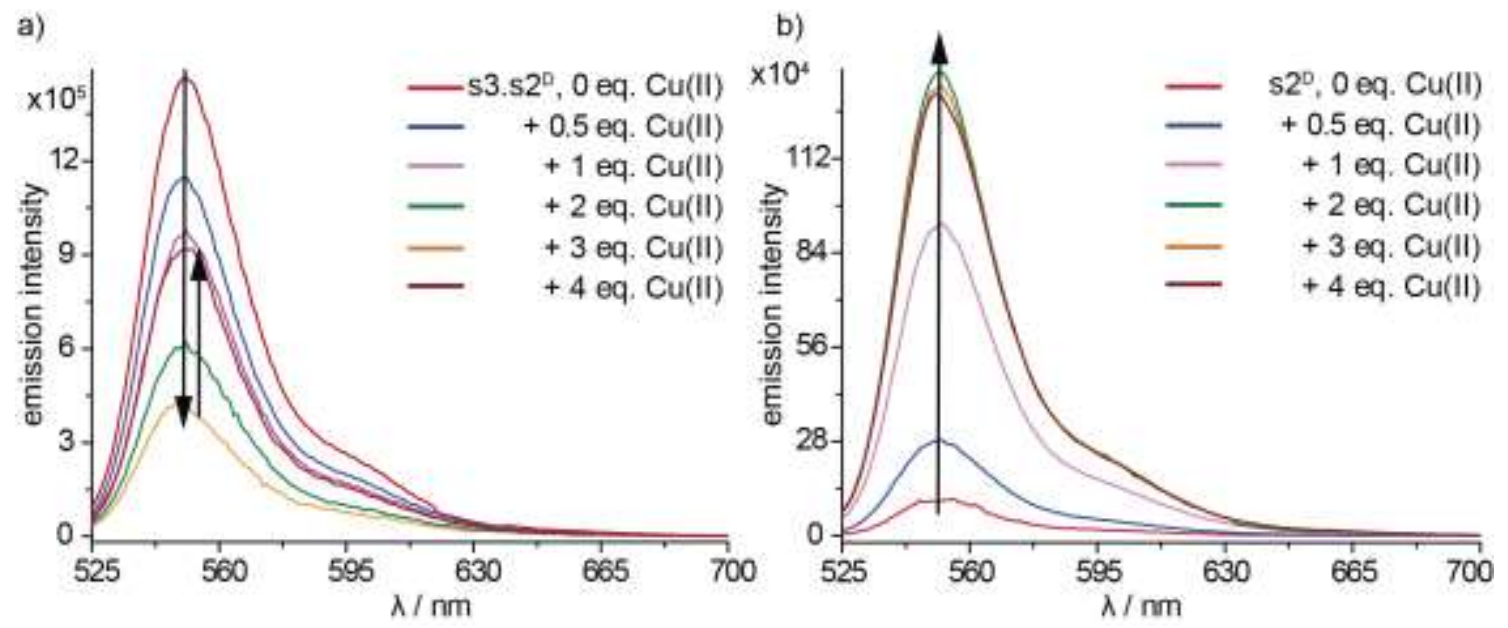

Figure 100. Appendix figure for emission spectra measured at excitation $515 \mathrm{~nm}$ and emission

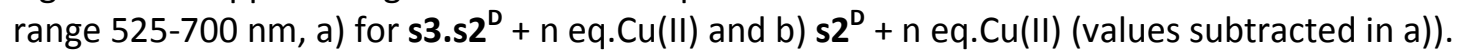
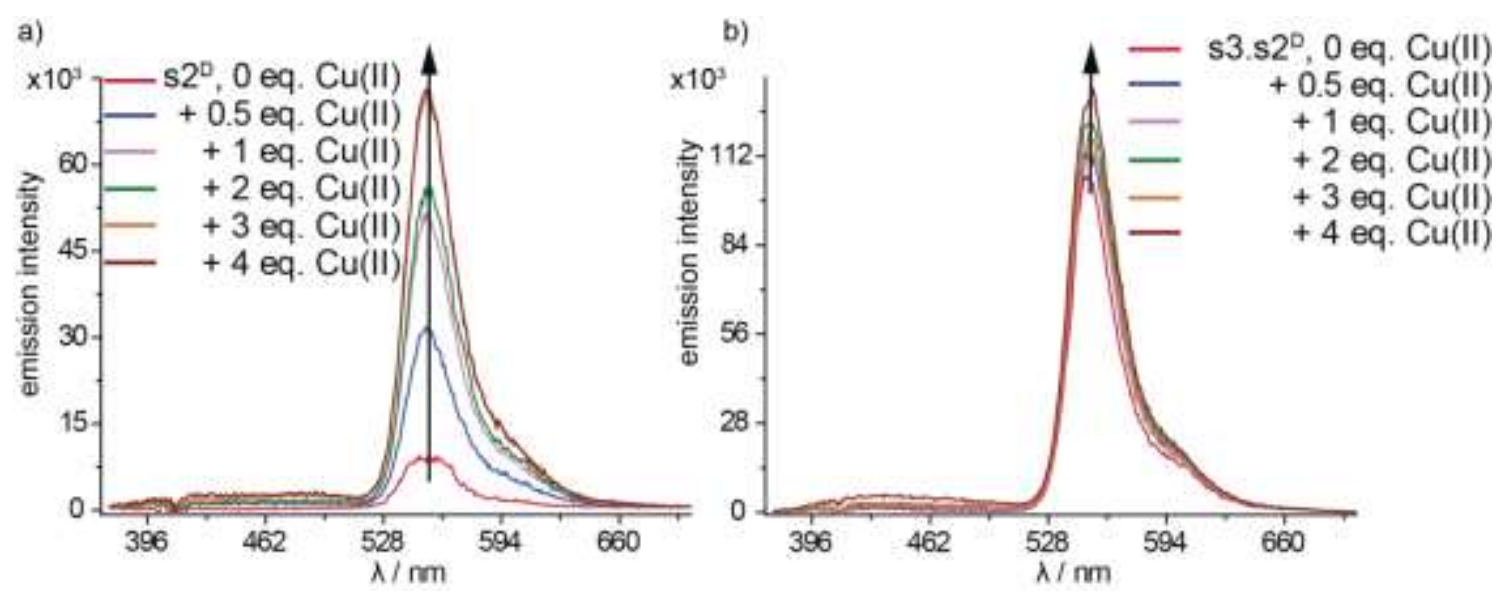

Figure 101. Appendix figure for emission spectra at excitation $360 \mathrm{~nm}$ and emission range 375-

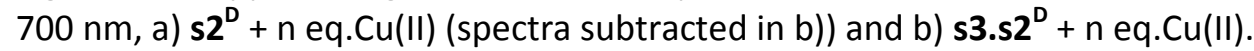

a)

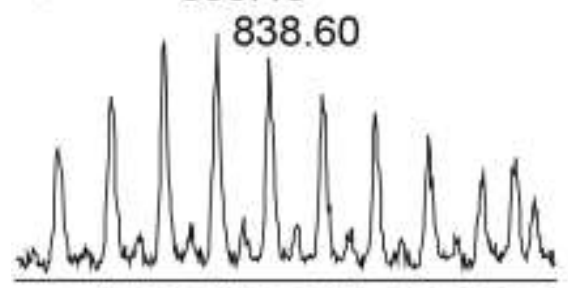

calc. $\left[\mathrm{s} 3+\mathrm{Ni}^{2+}-8 \mathrm{H}\right]^{-6}, 5027.78$

838.46

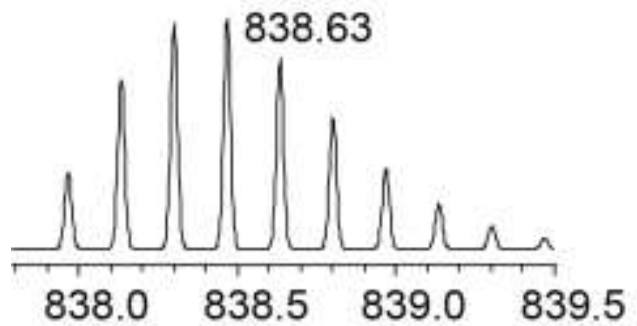

b)

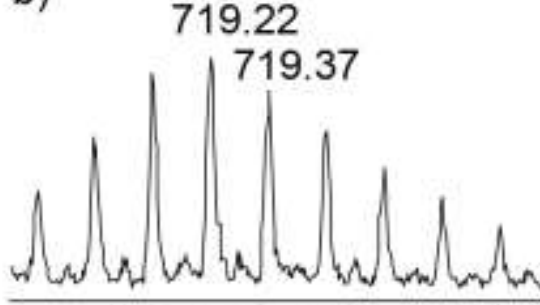

calc. $\left[\mathrm{s} 3+\mathrm{Cu}^{2+}-9 \mathrm{H}\right]^{-7}, 5031.77$

719.25

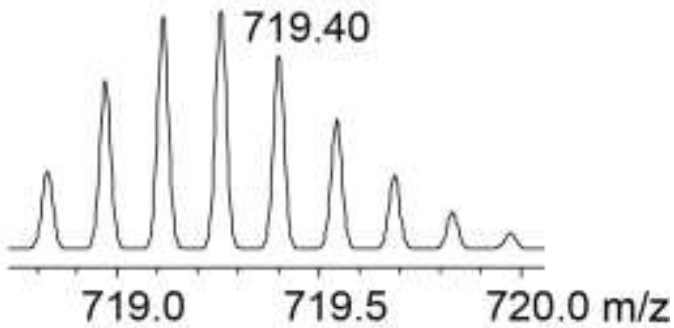

Figure 102. Appendix figure for ESI MS spectra indicating single strand $\mathbf{s} 3$ metallated a) with $\mathrm{Ni}(I I), \mathrm{m} / \mathrm{z}=-6$ and $\mathrm{b}$ ) with $\mathrm{Cu}(\mathrm{II}), \mathrm{m} / \mathrm{z}=-7$. 
a) 828.9413

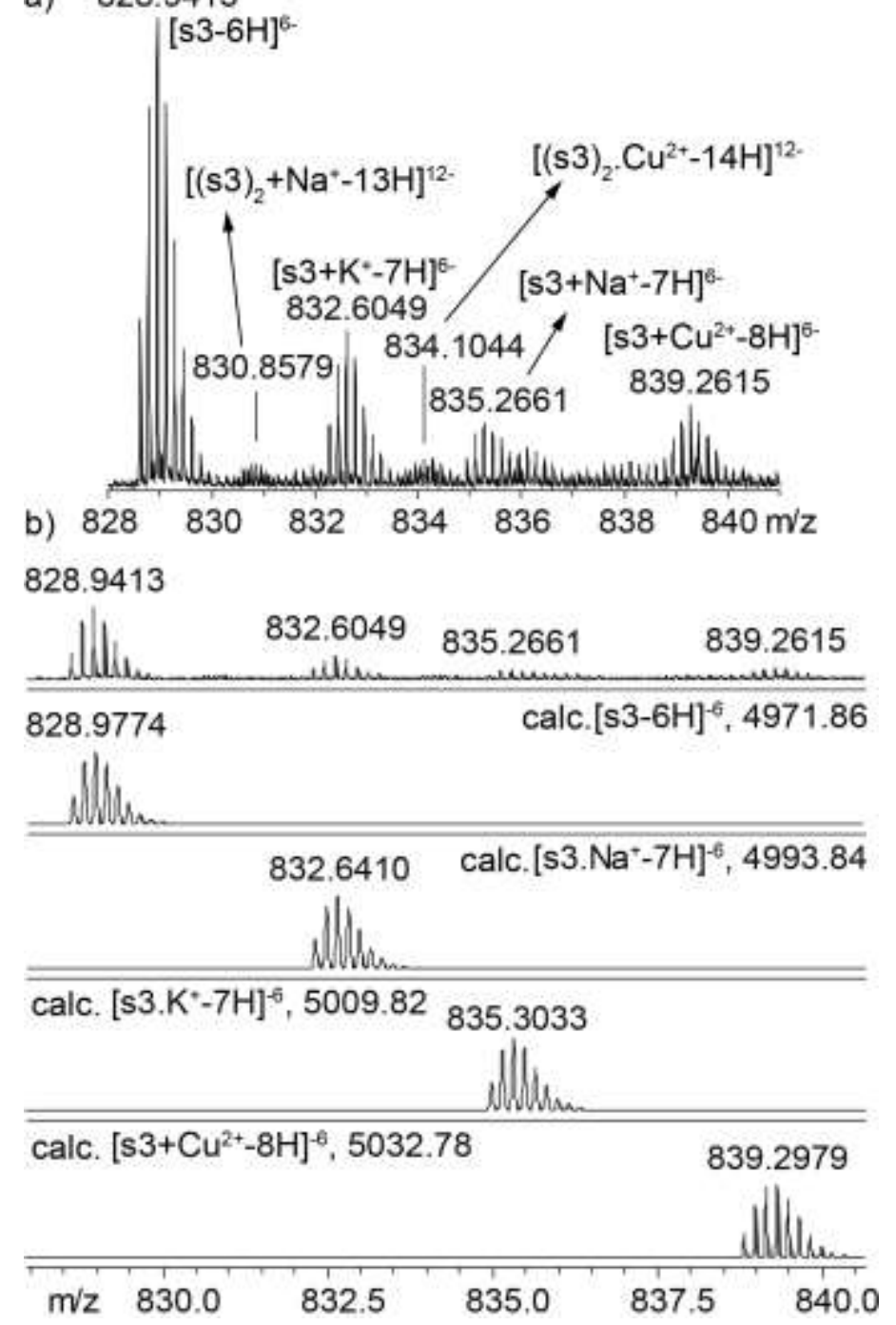

Figure 103. Appendix figure for ESI HRMS spectra indicating single strand $\mathbf{s} 3$ metallated with $\mathrm{Cu}(\mathrm{II})$, a) full range of spectrum with different adducts, b) measured and calculated pattern for $\mathrm{Cu}(\mathrm{II})$-DNA adducts at $\mathrm{m} / \mathrm{z}=-6$.

\subsection{Abbreviations}

A

Å

APS

$\mathrm{Bp}$

BHQ1

BTT

C

$C D$

CEP adenosine, absorbance

Angstrom $\left(1 \AA=10^{-10} \mathrm{~m}\right)$

solution of ammonium persulfate

base pair

black hole quencher 1

5-benzylthio-1H-tetrazole

cytidine

circular dichroism

2-cyanoethyl N,N-diisopropylchlorophosphoramidite 
CHES

CPG

dA

$\mathrm{dC}$

dG

dl

dT

dU

dIU

$d X$

DCM

DMAP

DMEDA

DMF

DMSO

DMT

DNA

$d s$-DNA

eq.

ESI

Et3N

F

FRET

h

HEPES

HEX

HIV-1

HPLC

HR

$\mathrm{Hz}$

iPr

J

$\mathrm{K}_{2} \mathrm{CO}_{3}$

M

$M(I I)$
$\mathrm{N}$-cyclohexyl-2-amino-ethanesulfonic acid

controlled pore glas

deoxyadenosine

deoxycytidine

deoxyguanosine

deoxyinosine

deoxythymine

deoxyuridine

deoxy5iodouridine

deoxy-artificial base

dichlro-methane

4-(dimethylamino)pyridine

$\mathrm{N}-\mathrm{N}$-diethylethylenediamine

dimethylformamide

dimethylsulphoxide

4,4'-dimethoxytrytil

deoxyribonucleic acid

double stranded DNA

equivalence

electronspray ionization

triethylamine

fluorescence

Förster resonance energy transfer

hour

4-(2-hydroxyethyl)piperazine-1-ethanesulfonic acid

donor fluorophore

Human imunodeficiency virus type 1

high performance liquid chromatography

high resolution

hertz

iso-propyl

coupling contant

potassium carbonate

molarity

dicationic metal specie 
Chapter 5

MALDI

Me

MeCN

Melm

$\mathrm{MeOH}$

MS

MW

$\mathrm{NaClO}_{4}$

$\mathrm{NaCl}$

ng

$\mathrm{nm}$

NMR

PAGE

ppm

py

$\mathrm{RP}$

r.t.

ss-DNA

SPS

T

TCA

TBM

TEAA

TEMED

THF

Tm

TP

u.a.

UV

X

$\mathrm{ZnF}$

$\theta$

$\delta$

$\varepsilon$
Appendix

Abbreviation List

matrix assisted laser desoption

methyl

acetonitrile

$\mathrm{N}$-methylimidazole

methanol

mass spectrometry

molecular weight

sodium perchlorate

sodium chloride

nanogram

nanometer

nuclear magnetic resonance

polyacrylamide gel electrophoresis

parts per million

pyridine

reversed phase

room temperature

single strand DNA

solid phase synthesis

temperature, thymidine

trichloroacetic acid

tris boric magnesium buffer

trimethylammonium acetate

$\mathrm{N}, \mathrm{N}, \mathrm{N}$ `,N`-tetramethylene diamine

tetrahidrofurane

melting temperature

triphosphate

units of absorbance

Ultraviolet

modified nucleotide incorporated

zinc finger

angular value, folded fraction

chemical shift

extinction coefficient 
micro

molar fraction

wavelength

\subsection{List of Figures}

Figure 1. Secondary structures for A-DNA, B-DNA and Z-DNA, respectively. 1

Figure 2. Conformations for $\mathrm{C}^{`}{ }^{`}$-endo (B-DNA), where the $2^{`}$-carbon lies above the pyranose ring, and $C 3^{`}$-endo (A-DNA) where $3^{`}$-carbon is above the pyranose ring and at the same side of 5 -carbon. . .2

Figure 3. Watson-Crick base pair for A-T, two hydrogen bondings, N6 and 04 and N1 and N3.. 3 Figure 4. Watson-Crick base pair for G-C, three hydrogen bondings, $\mathrm{O} 6$ and N4, N1 and N3 and $\mathrm{N} 2$ and $\mathrm{O} 2$. 3

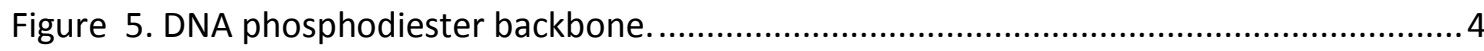

Figure 6. a) DNA triplex, b) Holliday junction and c) Hoogsteen interaction in a G-quartet........5 Figure 7. Scheme for a DNA Origami plan a) detailed plan for a monomeric retangular fold made with 66 body staples (blue) and edge staples (grey) hybridized into a template (green), b) Monomer template before and after annealed, c) Atomic force micrograph of respective monomer (50 nm). ${ }^{[16]}$

Figure 8. Three different modes of binding for complexes with $d s$-DNA, from left to right: groove binder, metallo-intercalator and metallo-insertor. Note that while metallo-intercalator interacts on major groove, metallo-insertor acts on minor groove, producing a stronger strain in the DNA due to replacement of the expelled base pairs after entering the DNA double helix. [21]

Figure 9. a) Selective intercalation of $\left[\mathrm{Rh}(\mathrm{phi})\left(\mathrm{Me}_{2} \text { trien }\right)\right]^{3+}$ into the major groove of the $d s$ DNA, 5`-TG/CA-3`. Phi= 9,10 -phenanthrenequinone diamine and $\mathrm{Me}_{2}$ trien= 2R,9R-diamino4,7-diazodecane. b) Specific hydrogen bonds between the intercalator and the oxygen atoms O6 of the guanines on the intercalation site, $3.0 \AA .{ }^{[24]}$ .8

Figure 10. Types of cross-links between cis-platinum and $d s-D N A$ a) interstrand cross-link b) 1,2-intrastrand cross-link, c) 1,3-intrastrand cross-link, d) scheme of a kink caused in the dsDNA due to the coordination of cis-platinum with $\mathrm{G}$ nucleotides on the DNA. ${ }^{[2]}$...................... 8 Figure 11. Examples of artificial nucleosides based on 5-iodo-2 '-deoxyuridine, dIU. a) phorphyrin group attached through 5-propargylamine linker to 2'-deoxyuridine. b) transchelating oxazol group attached through 5-alkyne linker to 2 '-deoxyuridine. For solid phase DNA synthesis further steps as DMT protection at the 5 -position and phosphoramidation at 3 '-position are required. More synthetic details are given in the experimental part. 9 
Figure 12. Examples of ligands incorporated into DNA a) trans-chelating type, [35] b) phenanthroline type. ${ }^{[36,37]}$ Building block required for SPS containing 5 `-DMT protected and 3 `phosphoramidation. 10

Figure 13. Scheme for modified DNA containing metal complexation between Fe(II), $\mathrm{Cu}(\mathrm{I})$ and $\mathrm{Cu}(\mathrm{II})$ with groups based on terpyridine and bipyridine in homoleptic and heteroleptic modified DNA duplexes. ${ }^{[37]}$.

Figure 14. Formation of cyclic [Zn(II).ss-DNA] structure. 12

Figure 15. Schematic detail for the DNA 3-way junction developed by Shionoya group. 12

Figure 16. Metal-base pairing between T-T mismatch and $\mathrm{Hg}(\mathrm{II})$ proposed by Katz. 13

Figure 17. a) Scheme for the salen-base pairing complex, b) DNA duplex in a stack with multiple $\mathrm{Cu}(\mathrm{II})$ and $\mathrm{Hg}(\mathrm{II})$ complexes.

Figure 18. Deprotected nucleosides based on tepy into dIU a) Wagenknecht approach, ${ }^{[56]}$ b) Stulz approach, ${ }^{[57]}$ c) illustrative idea how is the group on b) pointing outside of the ds-DNA. 15 Figure 19. Representation of a) dimer complex formed by interduplex cross-link between DNA due to metal coordination and b) dimeric complex between two modifications on the same $d s$ DNA. ${ }^{[30]}$ 16

Figure 20. Scheme outlining the process of solid phase DNA synthesis. 17

Figure 21. Melting curve profile for sharp $d s$-DNA transition (solid black line) and respective first derivative (solid gray line) it is possible to see, $\mathrm{T}_{\max } \approx \mathrm{Tm}$ for sharp transitions, $d s$-DNA deviations due to evaporation (red dashed line) and ss-DNA (dashed gray line). ${ }^{[3,59]}$ 22

Figure 22. Melting curve for a sharp transition and its melting point determined by folded fraction method, indicating lower and higher baseline selected. ${ }^{[60]}$ 22

Figure 23. Examples of treatments for thermal analysis curve for a sample (see section 2.2.4 for sample details and results) of our own work. ${ }^{[35]}$ a) Original melting curve profile (solid black line) and after double sigmoidal fit (dashed line); b) Melting points of the curve presented in a) based on the first derivative method (dashed line) and smoothed (solid line); c) First derivative method for curve treated by double sigmoidal fit (excluding intermediates state effects from the curve). d) Melting curve (solid black line) and its respective profile treated by folded fraction method (dashed line) indicating the melting point value obtained by folded fraction conversion $\left(\mathrm{Tm}=\mathrm{T}\right.$ at $\left.\theta=0.5, \mathrm{Tm}=47^{\circ} \mathrm{C}\right)$ and e) First derivative of the curve after conversion by folded fraction method...

Figure 24. Scheme for photon excitation and emission and vibrational relaxations. ............. 27

Figure 25. Figurative scheme for contact quenching and weaking coupling mechanisms....... 28

Figure 26. Scheme for photon absorption and emission of donor and acceptor................... 29

Figure 27. Scheme for strand displacement by addition of a target strand........................ 30 
Figure 28. Scheme for adjacent probes where single strands containing donor and acceptor are hybridized with a longer complementary strand in a way that both chromophores are placed closed to each other allowing weak coupling of energy transfer .31

Figure 29. Scheme for a molecular beacons probe, a hairpin structure containing a groundstate complex between donor and acceptor and a further unfolding of such structure due to addition of complementary strand promoting emission of the donor fluorofore caused by the distance of the acceptor

Figure 30 . Scheme for TaqMan ${ }^{\circledR}$ probe promoting cleavage of system with donor-acceptor pair.

Figure 31. Scheme for the self-assembly of trans-chelated square-planar Pd(II) complex with bent ligands as linkers, producing rings of different sizes and thermodynamic stabilities. ${ }^{[79]} . .39$ Figure 32. Reactional scheme for the monomeric complexes synthesized. a) Scheme for $L 2$ and $\mathrm{L} 2 \mathrm{c}$, electron rich derivatives which need precursor complex $\left[\mathrm{Pd}(\mathrm{L} 2-2 \mathrm{c}) \mathrm{Cl}_{2}\right]$ formation and $\left.\mathrm{b}\right)$ direct formation of $\left[\mathrm{Pd}(\mathrm{L} 2 \mathrm{~b})(\mathrm{MeCN})_{2}\right]^{2+}$ because of its electron deficiency. .40 Figure 33. ${ }^{1} \mathrm{H}$ NMR spectra for ligand $L 2$ and $L 2 b$, and their respectives monomer complexes with $\mathrm{Pd}(\mathrm{II})$ and $\mathrm{CD}_{3} \mathrm{CN}$ and the dimer complex of $\mathrm{L} 2$ .40

Figure 34. Scheme for assembly of 2 - and 3-membered rings from the respective ligands and $\operatorname{Pd}(I I)$ complexes. 41

Figure $35 .{ }^{1} \mathrm{H}$ NMR spectra for 2 - and 3- membered rings formed. 42

Figure 36. ESI mass spectra for RING1 and respective isotopic pattern, calculated and measured.

Figure 37 . Scheme for conversion of RING3 into the interpenetrated double-cage + dimer....43 Figure 38. ${ }^{1} \mathrm{H}$ NMR spectra indicating the convertion of the 2-memberd ring (RING3) to the interpenetrated double-cage $\left[2 \mathrm{Cl}+\mathrm{BF}_{4} @ \mathrm{Pd}_{4} \mathrm{~L}_{8}\right]\left(\mathrm{BF}_{4}\right)$ and dimer $\left[\mathrm{Pd}(\mathrm{L2})_{2}\right]\left(\mathrm{BF}_{4}\right)_{2}$

Figure 39. ESI spectra for RING3 and its convertion to the double cage after several days at room temperature.

Figure 40. Scheme for the desired dimer complexes, $\left[\mathrm{Fe}(\mathrm{L} 1)_{2}\right]^{2+},\left[\mathrm{Pd}(\mathrm{L2})_{2}\right]^{2+},\left[\mathrm{Cu}(\mathrm{L} 3)_{2}\right]^{1+}$, respectively. In ligand $\mathrm{L} 1, \mathrm{R}=-\mathrm{BrC}_{6} \mathrm{H}_{4}$. .45

Figure 41. ${ }^{1} \mathrm{H}$ NMR for dimer complexes in $\mathrm{CD}_{3} \mathrm{CN}$. .46

Figure 42. a) ESI-MS for dimer complexes together under same solution, b) Isotopic pattern for the calculated and measured peaks of each dimer. .47

Figure 43 . Scheme of synthetic route for trans-chelating ligand 5 and phosphoramidite 6. i) $\mathrm{K}_{2} \mathrm{CO}_{3}, \mathrm{MeCN}$, reflux 18h; ii) TMS-acetylene, THF, Cul, $\mathrm{PdCl}_{2}\left(\mathrm{PPh}_{3}\right)_{2}, \mathrm{Et}_{3} \mathrm{~N}$, r.t., 5h; iii) $\mathrm{K}_{2} \mathrm{CO}_{3}$, $\mathrm{MeOH}, 8$; iv) 1,2-diiodobenzene, $\mathrm{Cul}, \mathrm{PdCl}_{2}\left(\mathrm{PPh}_{3}\right)_{2}$, dry THF, $\mathrm{Et}_{3} \mathrm{~N}$, r.t., 8h; v) DMT-Cl, py, DMAP, 24h; vi) (iPr $2 \mathrm{~N})\left(\mathrm{OCH}_{2} \mathrm{CH}_{2} \mathrm{CN}\right) \mathrm{PCl}, \mathrm{NEt}(\mathrm{iPr})_{2}, \mathrm{THF}, 2 \mathrm{~h}$. 49 
Figure 44. Scheme for trans-chelating ligand incorporated into DNA and respective complementary single strands and $d s$-DNA formed and its respective metal-base pairing. .....50

Figure 45. Spartan modeling for modified ds-DNA containing MBP based on trans-chelating ligand and $\mathrm{M}(\mathrm{II}){ }^{\left[{ }^{[86}\right.}$ 50

Figure 46. a) Analytical chromatogram for s1 and s2 raw sequences indicating fail strands and other small impurities, b) chromatogram for s1 and s2 after purification and DMT cleavage. 52 Figure 47. Linear regression of plotted values of absorption at $260 \mathrm{~nm}$ for ligand 1,2-bis(2ethynyl-5-hydroxyethoxypyridine)benzene, 4.

Figure 48. ESI-MS for s1 and s2 single strands and calculated and measured isotopic pattern for $m / z=-9$. 54

Figure 49. UV/Vis spectra for ligand 4, "Lig." and respectives monomeric and dimeric complexes with $\mathrm{Pd}(\mathrm{II}), \quad(5.7 \mu \mathrm{M}$ in DMSO solution). Lig=1,2-bis(2-ethynyl-5-hydroxyethoxypyridine)benzene. 56

Figure 50. Melting curve analysis for the single strand s1 at 3.0, 4.5 and $6.0 \mu \mathrm{M}, \mathrm{b}$ ) plotting of Tm values vs concentration $(\mu \mathrm{M})$ indicating possible formation of a hairpin structure. $10 \mathrm{mM}$ cacodylate buffer, $100 \mathrm{mM} \mathrm{NaCl}$. c) UV/Vis for $3.0 \mu \mathrm{M}$ s1 at r.t. and $70^{\circ} \mathrm{C}$ indicating hyperchromism effect at $260 \mathrm{~nm}$. 57

Figure 51. a) Thermal analysis for $3.0 \mu \mathrm{M}$ unmodified duplex s1*.s2*, $10 \mathrm{mM}$ cacodylate buffer (pH 7.4), $100 \mathrm{mM} \mathrm{NaCl}$ and under same conditions melting curve for duplex s1.s2, $+\mathrm{n}$ eq. $\mathrm{Cu}(\mathrm{II})$. b) UV/Vis for $3.0 \mu \mathrm{M}$ s1.s2 + n eq. Cu(II), $10 \mathrm{mM}$ cacodylate buffer (pH 7.4), $100 \mathrm{mM}$ $\mathrm{NaCl}$. 57

Figure 52. a) Thermal analysis for a $3.0 \mu \mathrm{M}$ solution of the unmodified duplex s1*.s2*, $10 \mathrm{mM}$ cacodylate buffer ( $\mathrm{pH}$ 7.4), $100 \mathrm{mM} \mathrm{NaCl}, 10 \mathrm{mM} \mathrm{MgCl}_{2}$, and under same conditions melting curve for duplex s1.s2, + n eq. Cu(II). b) UV/Vis for $3.0 \mu \mathrm{M}$ s1.s2 + n eq. Cu(II), $10 \mathrm{mM}$ cacodylate buffer (pH 7.4), $100 \mathrm{mM} \mathrm{NaCl}, 10 \mathrm{mM} \mathrm{MgCl}$. 59 Figure 53. a) Thermal analysis for $3.0 \mu \mathrm{M}$ unmodified duplex $\mathrm{s} 1^{*} . \mathrm{s} 2^{*}, 10 \mathrm{mM}$ cacodylate buffer (pH 7.4), $100 \mathrm{mM} \mathrm{NaClO}_{4}$ and under the same conditions for melting curve of the duplex s1.s2, $+\mathrm{n}$ eq. $\mathrm{Pd}(\mathrm{II})$. b) UV/Vis for $3.0 \mu \mathrm{M}$ s1.s2 + n eq. Pd(II), $10 \mathrm{mM}$ cacodylate buffer (pH 7.4), $100 \mathrm{mM} \mathrm{NaClO}_{4}$

Figure 54. a) Thermal analysis of a $3.0 \mu \mathrm{M}$ solution of the unmodified duplex s1*.s2*, $10 \mathrm{mM}$ cacodylate buffer ( $\mathrm{pH}$ 7.4), $100 \mathrm{mM} \mathrm{NaClO}_{4}$ and under same conditions melting curve for duplex s1.s2, + n eq. Cu(II). b) UV/Vis for $3.0 \mu \mathrm{M}$ s1.s2 + n eq. Cu(II), $10 \mathrm{mM}$ cacodylate buffer (pH 7.4), $100 \mathrm{mM} \mathrm{NaClO}_{4}$.

Figure 55. Emission spectra for $3.0 \mu \mathrm{M}$ s1.s2 + n eq. M(II), $10 \mathrm{mM}$ cacodylate buffer (pH 7.4), a) $100 \mathrm{mM} \mathrm{NaCl}, \quad \mathrm{M}(\mathrm{II})=\mathrm{Cu}(\mathrm{II}), \quad$ b) $100 \mathrm{mM} \quad \mathrm{NaClO}_{4}, \mathrm{M}(\mathrm{II})=\mathrm{Cu}(\mathrm{II}), \quad$ c) $100 \mathrm{mM} \quad \mathrm{NaClO}_{4}$, $M(I I)=P d(I I)$. Excitation $330 \mathrm{~nm}$, emission $340 \mathrm{~nm}$. 62 
Figure 56. CD spectra for a) $3.0 \mu \mathrm{M} s 1$ (room temperature and at $70^{\circ} \mathrm{C}$ ), s2, and duplex s1.s2, $10 \mathrm{mM}$ cacodylate buffer (pH 7.4), $100 \mathrm{mM} \mathrm{NaCl}$, b) $3.0 \mu \mathrm{M}$ s1.s2 + n eq. Cu(II), $10 \mathrm{mM}$ cacodylate buffer (pH 7.4), $100 \mathrm{mM} \mathrm{NaCl}, 100 \mathrm{mM}$. Excitation wavelength $330 \mathrm{~nm}$, emission wavelength $340 \mathrm{~nm}$. .63

Figure 57. ESI-MS for $20 \mu \mathrm{M}$ s1.s2 and metal adducts with $\mathrm{Cu}(\mathrm{II})$. .64

Figure 58 . Native $8 \%$ polyacrylamide gel with TBM buffer $(\mathrm{pH} 8,3)$ for single strands $s 1$, s2 and duplex s1.s2. . .66

Figure 59. Native $8 \%$ polyacrylamide gel with TBM buffer $(\mathrm{pH} \mathrm{8.3)}$ for the single strands $\mathrm{s} 1, \mathrm{~s} 2$ and duplex s1.s2 and for the respectives attempts of $M(I I)$ complexation. .66

Figure 60 . Scheme for the trans-chelating artificial nucleotide pointing outside of the ss-DNA.

Figure 61. Synthetic path for designed nucleoside 7 and phosphoramidite 8. i) $\mathrm{I}_{2}, \mathrm{H}_{2} \mathrm{SO}_{4}, 40^{\circ} \mathrm{C}$, $1 \mathrm{~h}$; ii) ligand 2, Cul, $\mathrm{PdCl}_{2}\left(\mathrm{PPh}_{3}\right)_{2}$, THF, 2-ethynyl-pyridine, $\mathrm{Et}_{3} \mathrm{~N}$, r.t., $24 \mathrm{~h}$; iii) $\mathrm{K}_{2} \mathrm{CO}_{3}, \mathrm{Cul}$, toluene, p-amino-benzamide, DMEDA, $120^{\circ} \mathrm{C}, 48 \mathrm{~h}$; iv) i-amyl-ONO, $\mathrm{CH}_{2} \mathrm{I}_{2}, 48 \mathrm{~h}$; vii) ligands 4 and 6, Cul, $\mathrm{PdCl}_{2}\left(\mathrm{PPh}_{3}\right)_{2}, \mathrm{DMF}, 70^{\circ} \mathrm{C}, 16 \mathrm{~h}$; viii) ligand 9, dry THF, NEt( $\left.\mathrm{PPr}\right)_{2}$, $\left(\mathrm{iPr}_{2} \mathrm{~N}\right)\left(\mathrm{OCH}_{2} \mathrm{CH}_{2} \mathrm{CN}\right) \mathrm{PCl}, 2 \mathrm{~h}$. .68

Figure 62. Crystal structure for nucleoside 7. Distance between the $1^{\prime}-\mathrm{N}$-uridine until the binding site was highlighted in the figure. 69

Figure 63. a) Schematic view for the artificial s3 strand and interduplex cross-link for s3.s2*M(II). b) spartan modeling for the intercross linked nanoconstruct. ${ }^{[86]}$ . .70

Figure 64. a) Schematic picture of the modified complementary strands $s 4$ and s5 giving the doubly modified duplex s4.s5 and further DNA nanoconstruct upon addition of $M(I I)$. Spartan model with b) 11 base pairing of distance and c) 10 base pairing of distance between the artificial nucleotides. ${ }^{[86]}$ .71

Figure 65. HPLC chromatograms for s3, s4 and s5 strands, respectively (left side as crude materials and right side after purification and detritylation). .74

Figure 66. Linear regression of the plotted values of absorption at $260 \mathrm{~nm}$ for detritylated ligand 7 at dilutions from 0.07 to $0.003 \mathrm{mM} \mathrm{CH}_{3} \mathrm{Cl} / \mathrm{MeOH} 1: 1$ solution. Deviations of linearity started after $0.007 \mathrm{mM}$. .75

Figure 67. ESI-MS spectra of the characterization for single strands a) s3, b) s4 and c) s5......76 Figure 68. UV/Vis spectra of $3.0 \mu \mathrm{M}$ DNA duplex s3.s2*, $10 \mathrm{mM}$ cacodylate buffer (pH 7.4), $100 \mathrm{mM} \mathrm{NaCl}$ with addition of $\mathrm{n}$ eq. of $\mathrm{Cu}(\mathrm{II})$. .77

Figure 69. UV/Vis spectra of $3.0 \mu \mathrm{M}$ DNA duplex s3.s2*, $10 \mathrm{mM}$ cacodylate buffer (pH 7.4), $100 \mathrm{mM} \mathrm{NaClO}_{4}$ with addition of $\mathrm{n}$ eq. of $\mathrm{M}(\mathrm{II})$, a) $\mathrm{M}(\mathrm{II})=\mathrm{Pd}(\mathrm{II})$ and b) $\mathrm{M}(\mathrm{II})=\mathrm{Cu}(\mathrm{II})$. . .78

Figure 70. Melting curves for $3.0 \mu \mathrm{M}$ of DNA duplex s3.s2*, $10 \mathrm{mM}$ cacodylate buffer $(\mathrm{pH} 7.4)$ a) in comparison to unmodified $\mathrm{s} 1 * \mathrm{~s} 2 *$ duplex, both in salinic solution of $100 \mathrm{mM} \mathrm{NaCl}$ and b) 
$+\mathrm{n}$ additions of $\mathrm{Cu}(\mathrm{II})$ and melting curve profiles with $100 \mathrm{mM} \mathrm{NaClO}_{4}$ as electrolyte for the duplex s3.s2* with addition of c) $\mathrm{Pd}(\mathrm{II})$ and d) $\mathrm{Cu}(\mathrm{II})$. All the measurements with temperature rate of $0.5^{\circ} \mathrm{C} / \mathrm{min}$ 79

Figure 71. Comparison between the CD spectra and schematic squeme of the modified strands s1 and s3 containing incorporation of a trans-chelating ligand a) pointing outside of DNA and b) incorporated internally to the double helix. $3 \mu \mathrm{M}$ ss-DNA and ds-DNA, $10 \mathrm{mM}$ cacolylate buffer $(\mathrm{pH} 7.4)$ and $100 \mathrm{mM} \mathrm{NaCl}$......

Figure 72. CD specra for a) ss-DNA $s 3, s 2^{*}$ and the duplex $s 3 . s 2^{*}$, b) CD spectra for the DNA duplex after addition of $\mathrm{Cu}(\mathrm{II})$ equivalents. $3 \mu \mathrm{M}$ ss-DNA and ds-DNA, $10 \mathrm{mM}$ cacodylate buffer (pH 7.4), $100 \mathrm{mM} \mathrm{NaCl}$.

Figure 73. CD specra for a) ss-DNA s3, $s 2^{*}$ and the duplex $s 3 . s 2^{*}$, b) CD spectra for the DNA duplex after addition of $\mathrm{Cu}(\mathrm{II})$ and $\mathrm{c}$ ) after addition of $\mathrm{n}$ eq. of $\mathrm{Pd}(\mathrm{II}) .3 \mu \mathrm{M} s s$-DNA and ds-DNA, $10 \mathrm{mM}$ cacodylate buffer ( $\mathrm{pH}$ 7.4), $100 \mathrm{mM} \mathrm{NaClO}_{4}$.

Figure 74. Emission spectra for a) $3 \mu \mathrm{M} \mathrm{s3.s2*,10} \mathrm{mM} \mathrm{cacodylate} \mathrm{buffer} \mathrm{(pH} \mathrm{7.4),} 100 \mathrm{mM}$ $\mathrm{NaCl}+\mathrm{n}$ eq. of $\mathrm{Cu}(\mathrm{II})$ and $3 \mu \mathrm{M}$ s3.s2*, $10 \mathrm{mM}$ cacodylate buffer ( $\mathrm{pH}$ 7.4), $100 \mathrm{mM} \mathrm{NaClO}{ }_{4}+$ $\mathrm{n}$ eq. of $\mathrm{M}(\mathrm{II})$, b) with $\mathrm{M}(\mathrm{II})=\mathrm{Pd}(\mathrm{II})$ and $\mathrm{c}$ ) with $\mathrm{M}(\mathrm{II})=\mathrm{Cu}(\mathrm{II})$. Excitation wavelength at $360 \mathrm{~nm}$, width $2 \mathrm{~nm}$

Figure 75. Scheme for inter cross-link between duplexes formed through dimer metal complexation and possible units, $A$ and $B$, with their respective figurative intensities of emission. 84

Figure 76. Fluorescence spectra for the DNA duplexes $s 3 . s 2^{*}$ and $s 3 . s 2^{\mathrm{D}}$ and further additions of $\mathrm{Cu}(\mathrm{II})$ to the duplex $s 3 . s 2^{\mathrm{D}}$. Equivalents of $\mathrm{Cu}(\mathrm{II})$ are showed in the respective color of the lines in the spectra. a) View of the entire spectra, b) zoom of the range 525-600 nm related to the range of emission for the FRET pair Hex/BHQ1. Excitation $360 \mathrm{~nm}$, emission range 375$650 \mathrm{~nm}$, width $2 \mathrm{~nm}$. Sample conditions: $3.0 \mu \mathrm{M}$ ds-DNA, $10 \mathrm{mM}$ cacodylate buffer (pH 7.4), $100 \mathrm{mM} \mathrm{NaCl}$.

Figure 77. ESI-HRMS of $20 \mu \mathrm{M} s s-D N A$ s3 adducts with $\mathrm{Cu}(\mathrm{II}), \mathrm{H}_{2} \mathrm{O} / \mathrm{MeOH}$ (50:50). a) Unmetallated and metallated dimers $\mathrm{m} / \mathrm{z}=-12$, b) Pattern for $\operatorname{dimer}\left[(\mathrm{s} 3)_{2} \mathrm{Cu}^{2+}-13 \mathrm{H}\right]^{-11}$. Stacking for the spectrum of dimeric adducts $c) m / z=-13$ and d) $m / z=-11$.

Figure 78. ESI-HRMS of $20 \mu \mathrm{M}$ ss-DNA s3 adducts with $\mathrm{Ni}(\mathrm{II}), \mathrm{H}_{2} \mathrm{O} / \mathrm{MeOH}$ (50:50). a) Spectrum of the dimeric unmetallated and metallated adducts of the DNA inter cross-linked $m / z=-13$, b) Stacking of the spectrum of the dimeric adducts and calculated respective isotopic pattern for $m / z=-11$. 88

Figure 79. Native TBM gel $8 \%$ for 500 ng s3.s2* with M(II). a) Visualization of the full gel after staining with use of sepia filter, b) Close-up picture for lines 2 to 5 before staining, indicating blue fluorescence color from the single strand s3, c) Closed-up picture for lines 2 to 5 after 
staining and converted to black and white. Line 1: ss-DNA s2*, line 2: ss-DNA s2*+ ss-DNA s3, line 3: ss-DNA s2*+ss-DNA s3 + 0.5 eq. Cu(II), line 4: ss-DNA s2*+ss-DNA s3 + 0.5 eq. Ni(II), line 5: ss-DNA s2*+ss-DNA s3 + 1.0 eq.Cu(II). The lines before line 1 and after line 5 were loaded with samples containing DNA ladder (150-10bp). 91

Figure 80. Native 8\% TBM gel for 500 ng s3.s2* with M(II). Line 1 and 8: DNA ladder 150-10bp. Line 2: ss-DNA s3, line 3: ss-DNA s2*, line 4: ss-DNA s2*+ss-DNA s3, line 5: ss-DNA s2*+ss-DNA s3 + 0.5 eq. Cu (II), line 6: ss-DNA s2*+ss-DNA s3 + 0.5 eq.Pd(II), line 6: ss-DNA s2*+ss-DNA s3 +0.5 eq. $\mathrm{Ni}(\mathrm{II})$. 91

Figure 81. Native 8\% TBM gel for 500 ng s3 with M(II). Line 1 and 8: DNA ladder 150-10bp. Line 2: ss-DNA s3, line 3: ss-DNA s3 + 0.5eq. Pd(II), line 4: ss-DNA s3 + 0.5eq.Cu(II), line 5: ss-DNA s3 +0.5 eq. $\mathrm{Ni}(\mathrm{II})$, line $6:$ ss-DNA s3 +1.0 eq. $\mathrm{Cu}(\mathrm{II})$, line $6:$ ss-DNA s3 + 1.0 eq. $\mathrm{Ni}(\mathrm{II})$.

Figure 82. Scheme for the formation of the unit (c) of inter cross-link between two duplexes s4.s5 a) hybridization of the duplex s4.s5, followed by the $M(I I)$ complexation, b) metal complexation of the melted system followed by hybridization of $\$ 4.55$ under slow cooling producing the unit given in $\mathrm{c}$ ). 93

Figure 83. Scheme of possible spinning of the unhybridized system and final polymer product as a result of a fast cooling hybridization. 94

Figure 84. UV/Vis spectra for $4 \mu \mathrm{M}$ s4.s5, $150 \mathrm{mM} \mathrm{NaClO}_{4}, 50 \mathrm{mM} \mathrm{CHES} \mathrm{buffer}+\mathrm{n}$ eq. of $\mathrm{Pd}(\mathrm{II})$. 95

Figure 85. UV/Vis spectra for $4 \mu \mathrm{M}$ s4.s5, $150 \mathrm{mM} \mathrm{NaClO}_{4}, 50 \mathrm{mM} \mathrm{CHES} \mathrm{buffer}+\mathrm{n}$ eq. of $\mathrm{Pd}(\mathrm{II})$.

Figure 86. a) UV/Vis spectra for different additions of $\mathrm{Cu}(\mathrm{II})$ amounts into the system s4.s5 until 2 eq. of $\mathrm{Cu}(\mathrm{II})$. b) UV/Vis spectra showing the difference in the sample concentration for the sample before (solid line) and after (dashed line) submitted to slow cooling for annealing process to avoid polymerization.

Figure 87. Thermal analysis for $4 \mu \mathrm{M}$ s4.s5 and s4*.s5*, $50 \mathrm{mM}$ CHES buffer, a) $150 \mathrm{mM}$ $\mathrm{NaClO}_{4}$, b) $150 \mathrm{mM} \mathrm{NaCl}$. Values in parenthesis are for the reannealing process, temperature rate of $0.1^{\circ} \mathrm{C} / \mathrm{min}$. 98

Figure 88. Thermal analysis for DNA duplexes a) s4.s5 and s4*.s5*(solid black line for melting process and dashed black line for renaturing process), b) melting processes for several cycles of s4.s5 upon addition of 1 eq. of $\mathrm{Pd}(\mathrm{II}), \mathrm{c})$ reannealing process for several cycles of s4.s5 upon addition of 1 eq. of $\mathrm{Pd}(\mathrm{II})$.

Figure 89. Thermal analysis for $4 \mu \mathrm{M}$ s4.s5, $150 \mathrm{mM} \mathrm{NaClO}_{4}, 50 \mathrm{mM}$ CHES buffer, upon additions of $\mathrm{Pd}(\mathrm{II})$, a) 1 eq, b) 2 eq, c) 3 eq.

Figure 90. CD spectra for $4 \mu \mathrm{M}$ of a) s4, s5 and s4.s5, $150 \mathrm{mM} \mathrm{NaClO}, 50 \mathrm{mM} \mathrm{CHES} \mathrm{buffer} \mathrm{and}$ b) CD spectra for $s 4 . s 5+n$ eq. of $\mathrm{Pd}(\mathrm{II})$. 101 
Figure 91. CD spectra for $4 \mu \mathrm{M}$ of a) s4, s5 and s4.s5, $150 \mathrm{mM} \mathrm{NaCl}, 50 \mathrm{mM}$ CHES buffer and b) CD spectra for $s 4 . s 5+n$ eq. of $\mathrm{Cu}(\mathrm{II})$.

Figure 92. Emission spectra for $4 \mu \mathrm{M}$ s4.s5, $50 \mathrm{mM} \mathrm{CHES} \mathrm{buffer,} \mathrm{a)} 150 \mathrm{mM} \mathrm{NaClO}_{4}$ and b) $150 \mathrm{mM} \mathrm{NaCl}$ upon addition of $\mathrm{Pd}(\mathrm{II})$ and $\mathrm{Cu}(\mathrm{II})$ respectively.

Figure 93. Native 8\% polyacrylamide gel in TBM buffer. Line 1 and 7: DNA ladder, line 2: single strand s4, line 3: double strand s4.s5, line 4: s4.s5+Cu(II) under slow cooling, line 5: s4.s5 + Ni(II), line 6: s4.s5 + Cu(II) under fast cooling. 103

Figure 94. Attempts to obtain evidence of $(\mathrm{s} 4 . \mathrm{s5})_{2} \mathrm{M}(\mathrm{II})$. Native $8 \%$ polyacrylamide gels in TBM buffer. a) samples submitted to fast cooling, b) samples submitted to an intermediate cooling and c) slow cooling indicating still some traces of polymerization. 105 Figure 95. Native 8\% polyacrylamide gel in TBM buffer. Line 1 and 8: DNA ladder, line 2: single strand s4, line 3: double strand s4.s5, line 4: s4.s5+1 eq. Cu(II) (slow cooling), line 5: s4.s5 + 1 eq. $\mathrm{Ni}(\mathrm{II})$ (slow cooling), line 6: s4.s5 + 2 eq. $\mathrm{Cu}(\mathrm{II})$ (slow cooling), line 7: s4.s5 + 1 eq. $\mathrm{Cu}$ (II) (fast cooling). 106

Figure 96. Crystal structure for the trans-chelating nucleoside by Dr. Mark Light in Southampton University, UK. 122

Figure 97. Appendix figure showing the modeling (Gaussian 09) for a) 3D membered RING1 with $\left\{\mathrm{Pd}_{3}(\mathrm{~L} 2)_{3}\right\}$ fragments and b) $2 \mathrm{D}$ membered RING3 with $\left\{\mathrm{Pd}_{2}\left(\mathrm{~L}_{2}\right)_{2}\right\}$ fragments. 128

Figure 98. Appendix figure of $\mathrm{CD}$ spectra for $3 \mu \mathrm{M}$ DNA, $100 \mathrm{mM} \mathrm{NaClO}, 10 \mathrm{mM}$ cacodylate buffer, a) s1, s2, s1.s2, b) s1.s2 + neq. Cu(II). 129

Figure 99. Appendix fugure for thermal analysis for duplex $3 \mu \mathrm{M} \mathrm{s3.s2*,100} \mathrm{mM} \mathrm{NaCl,} 10 \mathrm{mM}$ cacodylate buffer $+\mathrm{n}$ eq. of $\mathrm{Cu}(\mathrm{II})$. Temperature rate $0.1^{\circ} \mathrm{C} / \mathrm{min}$ 129

Figure 100. Appendix figure for emission spectra measured at excitation $515 \mathrm{~nm}$ and emission range $525-700 \mathrm{~nm}$, a) for $\mathrm{s} 3 . \mathrm{s} 2^{\mathrm{D}}+\mathrm{n}$ eq.Cu(II) and b) $\mathrm{s} 2^{\mathrm{D}}+\mathrm{n}$ eq.Cu(II) (values subtracted in a)).

Figure 101. Appendix figure for emission spectra at excitation $360 \mathrm{~nm}$ and emission range 375$700 \mathrm{~nm}$, a) $s 2^{\mathrm{D}}+\mathrm{n}$ eq.Cu(II) (spectra subtracted in b)) and b) $s 3 . s 2^{\mathrm{D}}+\mathrm{n}$ eq.Cu(II)..... 130

Figure 102. Appendix figure for ESI MS spectra indicating single strand s3 metallated a) with $\mathrm{Ni}(\mathrm{II}), \mathrm{m} / \mathrm{z}=-6$ and $\mathrm{b})$ with $\mathrm{Cu}(\mathrm{II}), \mathrm{m} / \mathrm{z}=-7$. 130

Figure 103. Appendix figure for ESI HRMS spectra indicating single strand s3 metallated with $\mathrm{Cu}(\mathrm{II})$, a) full range of spectrum with different adducts, b) measured and calculated pattern for $\mathrm{Cu}(\mathrm{II})-\mathrm{DNA}$ adducts at $\mathrm{m} / \mathrm{z}=-6$. 131

\subsection{List of Tables}

Table 1. Comparison between MALDI and ESI-MS. 34 
Table 2. Melting point values and conditions for several systems.

Table 3. Percentage for quenching upon metal addition ................................................62

Table 4. DNA sequences for the synthesized single strands. $X$ as artificial nucleotide.............72

Table 5. Melting point values for duplex s3.s2* upon addition of $\mathrm{M}(\mathrm{II})$.................................79

Table 6. Percentage of quenched emission values (maximum) for the duplex s3.s2*, $10 \mathrm{mM}$ cacodylate buffer, $100 \mathrm{mM} \mathrm{NaCl}$ or $\mathrm{NaClO}_{4}$ with different equivalents of $\mathrm{M}(\mathrm{II})$ added. ${ }^{[66]}$ .83

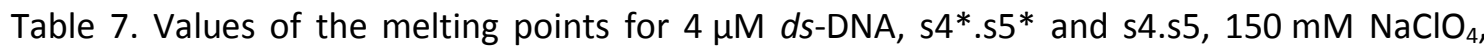
$50 \mathrm{mM}$ CHES buffer, upon addition of $\mathrm{Pd}(\mathrm{II})$. * in comparison to the unmodified duplex $\mathrm{s} 4^{*} . \mathrm{s5} 5^{*}$ 100

Table 8. Percentage quenching of s4.s5 system upon addition of $M(I I)$. 102

Table 9. Crystallographic data for compound 7 .123

Table 10. Values for preparation of the phosphoramidites. 124

Table 11. Details about the solutions for SPS. 124

Table 12. Amounts of reagents for the GEL preparation. Values for $10 \mathrm{~mL}$ of solution. 127

\section{References}

[1] J. D. Watson, F. H. C. Crick, Nature 1953(171), 737-738.

[2] ATDBio Nucleic Acids Book, www.atdbio.com/nucleic-acids-book.

[3] M. J. Hannon, Chem. Soc. Rev. 2007, 36(2), 280-295.

[4] C. R. Cantor and P. R. Schimmel, Biophysical Chemistry: Part 2: Techniques for the Study of Biological Structure and Function; W. H. Freeman, 1980.

[5] C. R. Cantor and P. R. Schimmel, Biophysical Chemistry: Part 1: The Conformation of Biological Macromolecules; W. H. Freeman, 1980.

[6] S. Zamenhof, G. Brawerman and E. Chargaff, Biochim. et Bioph. Acta - Specialized Section on Nucleic Acids and Related Subjects 1952, 9, 402-405.

[7] M. L. Bochman, K. Paeschke, V. A. Zakian, Nature Rev. Genet. 2012, 13(11), 770-780.

[8] E. Stulz, G. Clever, M. Shionoya, C. Mao, Chem. Soc. Rev. 2011, 40(12), 5633-5635.

[9] T. J. Bandy, A. Brewer, J. R. Burns, G. Marth, T. Nguyen, E. Stulz, Chem. Soc. Rev. 2011, 40(1), 138-148.

[10] R. W. Roberts and D. M. Crothers, Science 1992, 258, 1463-1466.

[11] S. M. Mirkin, and M. D. Frank-Kamenetskii, Annu. Rev. Biophys. Biomol. Struct. 1994, 23, 541-576.

[12] G. P. Pfeifer, A. Besaratinia, Photochem. Photobiol. Sci, 2012, 11(1), 90-97.

[13] L. Hu, C.-H. Lu, I. Willner, Nano letters 2015, 15(3), 2099-2103.

[14] P. Sa-Ardyen, A. V. Vologodskii, N.C. Seeman, Biophys. J., 2003, 84, 3829-3837.

[15] P. W. K. Rothemund, Nature 2006, 440(7082), 297-302.

[16] D. Han, S. Pal, J. Nangreave, Z. Deng, Science 2011, 332(6027), 342-346.

[17] K. E. Dunn, F. Dannenberg, T. E. Ouldridge, M. Kwiatkowska, A. J. Turberfield, J. Bath, Nature 2015, 525(7567), 82-86.

[18] E. R. Jamieson, S. J. Lippard, Chem. Rev. 1999, 99(9), 2467-2498. 
[19] a) R-Ann Musah, Curr. Top. Med. Chem., 2004, 4, 1605-1622; b) M. C. Williams, R. J. Gorelick, and K. Musier-Forsyth, PNAS 2002, 99(13), 8614-8619.

[20] S. F. Bellon, J. H. Coleman, and S. J. Lippard, Biochemistry 30, 1991, 8026-8035.

[21] a) J.K. Barton and S. J. Lippard, Biochemistry 1979, 18(12), 2661-2668; b) M. Howe-Grant, Kun C. Wu, W. R. Bauer, and S. J. Lippard, Biochemistry 1976, 15(19), 4339-4346.

[22] B. M. Zeglis, V. C. Pierre, J. K. Barton, Chem. Comm., 2007(44), 4565-4579.

[23] a) A. A. Gorodetsky, M. C. Buzzeo, J. K. Barton, Bioconjugate chemistry 2008, 19(12), 2285-2296; b) C.J. Murphy, M.R. Arkin, Y. Jenkins, N.D. Ghatlia, S.H. Bossmann, N.J. Turro, J.K. Barton, Science 1993, 262, 1025-1029; c) M. A. O'Neill, J. K. Barton, Topics in Current Chemistry; (Ed. G. Schuster), Springer Berlin Heidelberg, Berlin, Heidelberg, 2004, pp. 67115.

[24] D. J. Hurley, Y. Tor, J. Am. Chem. Soc. 2002, 124(14), 3749-3762.

[25] C. L. Kielkopf, K. E. Erkkila, B. P. Hudson, J. K. Barton and D. C. Rees, Nature 2000, 7(2), 117-121.

[26] a) G. L. Cohen, W. R. Bauer, J. K. Barton and S. J. Lippard, Science 1979, 203(4384), 10141016; b) J-P. Macquet and J-L. Butour, Biochemie, 1978, 60, 901-914; c) W. M. Scovell, L. R. Kroos, Biochem. Biophys. Res. Commun. 1982, 104, 1597-1603.; d) J. H. J. den Hartog, C. Altona, J. H. van Boom, G. A. van der Marel, C. A. Haasnoot, J. Reedijk, J. Biomol. Struct. dyn., 1985, 2(6), 1137-1155; e) J. H. J. den Hartog, C. Altona, G. A. van der Marel, J. Reedijk, Eur. J. Biochem. 1985(147), 371-379; f) F. Herman, J. Kozelka, V. Stoven, E. Guittet, J.-P. Girault, T. Huynh-Dinh, J. Igolen, J.-Y. Lallemand, J.-C. Chottard, Eur. J. Biochem. 1990, 194(119-133).

[27] C. A. Lepre, K. G. Strothkamp, and S. J. Lippard, Biochemistry 1987, 26, 5651-5657.

[28] E. Stulz, G.H. Clever (Eds), DNA in Supramolecular Chemistry and Nanotechnology; John Wiley \& Sons, 2015.

[29] A. Brewer, G. Siligardi, C. Neylon, E. Stulz, Org. Biomol. Chem. 2011, 9(3), 777-782.

[30] J. R. Burns, J. Zekonyte, G. Siligardi, R. Hussain, E. Stulz, Molecules, 2011, 16(6), 49124922.

[31] a) J. S. Choi, C. W. Kang, K. Jung, J. W. Yang, Y.-G. Kim, H. Han, J. Am. Chem. Soc. 2004, 126(28), 8606-8607; b) D. J. Hurley, S. E. Seaman, J. C. Mazura, Y. Tor, Org. Lett. 2002, 4(14), 2305-2308; c) H. Weizman, Y. Tor, J. Am. Chem. Soc. 2001, 123(14), 3375-3376.

[32] L. Kalachova, R. Pohl, L. Bednárová, J. Fanfrlík, M. Hocek, Org. Biomol. Chem. 2013, 11(1), 78-89.

[33] C. R. Cantor and P. R. Schimmel, Biophysical Chemistry: Part 3: The Behavior of Biological Macromolecules; W. H. Freeman, 1980.

[34] G. Clavé, G. Chatelain, A. Filoramo, D. Gasparutto, C. Saint-Pierre, E. Le Cam, O. Piétrement, V. Guérineau, S. Campidelli, Org. Biomol. Chem. 2014, 12(17), 2778-2783.

[35] J. R. Burns, K. Göpfrich, J. W. Wood, V. V. Thacker, E. Stulz, U. F. Keyser, S. Howorka, Angew. Chem. Int. Ed. 2013, 52(46), 12069-12072.

[36] C. K. McLaughlin, G. D. Hamblin, H. F. Sleiman, Chem. Soc. Rev. 2011, 40(12), 5647-5656.

[37] F. A. Pereira, G. H. Clever, Inorg. Chim. Acta 2016.

[38] H. Yang, H. F. Sleiman, Angew. Chem. Int. Ed.2008, 47(13), 2443-2446.

[39] H. Yang, A. Z. Rys, C. K. McLaughlin, H. F. Sleiman, Angew. Chem. Int. Ed. 2009, 48(52), 9919-9923.

[40] H. Yang, F. Altvater, De Bruijn, A Dowine, C. K. McLaughlin, P. K. Lo, H. F. Sleiman, Angew. Chem. Int. Ed. 2011, 50(20), 4620-4623. 
[41] G. H. Clever, T. Carell, Angew. Chem. Int. Ed.2007, 46(1-2), 250-253.

[42] G. Bianké, V. Chaurin, M. Egorov, J. Lebreton, E. C. Constable, C. E. Housecroft, R. Häner, Bioconjugate chemistry 2006, 17(6), 1441-1446.

[43] B.D. Hall, R.E. Holmlin, J.K. Barton, Nature 1996, 382.

[44] M. Göritz, R. Krämer, J. Am. Chem. Soc. 2005, 127(51), 18016-18017.

[45] J. Qiu, A. H. El-Sagheer, T. Brown, Chem. Comm. 2013, 49(62), 6959-6961.

[46] D. Mitra, N. Di Cesare, H. F. Sleiman, Angew. Chem. Int. Ed.2004, 43(43), 5804-5808.

[47] J-L. Duprey, Y. Takezawa, M. Shionoya, Angew. Chem. Int. Ed. 2013, 52(4), 1212-1216.

[48] E. Buncel, C. Boone, H. Joly, R. Kumar, and A. R. Norris, J. Inorg. Biochem. 1985, 25, 6173.

[49] S. Katz, Biochim. Biophys. Acta 1963, 68, 240-253.

[50] G. H. Clever, C. Kaul, T. Carell, Angew. Chem. Int. Ed. 2007, 46(33), 6226-6236.

[51] C. K. McLaughlin, G. D. Hamblin, H. F. Sleiman, Chem. Soc. Rev. 2011, 4O(12), 5647-5656.

[52] K.Tanaka and M. Shionoya, J. Org. Chem. 1994, 64, 5002-5003.

[53] S. Atwell, E. Meggers, G. Spraggon, and P. G. Schultz, J. Am. Chem. Soc. 2001, 123, 12364-12367.

[54] E. Meggers, P. L. Holland, W. B. Tolman, F. E. Romesberg, P. G. Schultz, J. Am. Chem. Soc. 2000, 122(43), 10714-10715.

[55] G. H. Clever, K. Polborn, T. Carell, Angew. Chem. Int. Ed. 2005, 44(44), 7204-7208.

[56] K. Tanaka, A. Tengeiji, T. Kato, N. Toyama, M. Shiro, M. Shionoya, J. Am. Chem. Soc. 2002, 124(42), 12494-12498.

[57] K. Tanaka, G. H. Clever, Y. Takezawa, Y. Yamada, C. Kaul, M. Shionoya, T. Carell, Nature Nanotec. 2006, 1(3), 190-194.

[58] T. Ehrenschwender, A. Barth, H. Puchta, H.-A. Wagenknecht, Org. Biomol. Chem. 2012, 10(1), 46-48.

[59] S. Verma and F. Eckstein, Annu. Rev. Biochem. 1998, 67, 99-134.

[60] J-L. Mergny and L. Lacroix, Oligonucleotides 2003, 13(23), 515-537.

[61] J.-L. Mergny, L. Lacroix, Curr Protoc Nucleic Acid Chem., edited by Serge L. Beaucage ... [et al.] 2009, Chapter 17, Unit 17.1.

[62] C.L. Stevens and G. Felsenfeld, Biopolymers 1964, 2, 293-314.

[63] J. D. Puglisi, I. Tinoco in RNA Processing Part A: General Methods, Methods in Enzymology, Elsevier, 1989, pp. 304-325.

[64] W. B. Melchior, JR. and P. H. Von Hippel, Proc. Nat. Acad. Sci. USA 1973, 70(2), 298-302.

[65] W. Beers, A. Cerami, and E. Reich 1967, 58, 1624-1631.

[66] M.E. Craig, D.M. Crothers, and P. Doty, J. Mol. Biol. 1971, 62, 383-401.

[67] V. V. Didenko, Fluorescent energy transfer nucleic acid probes: Designs and protocols; Humana Press, Totowa, N.J, 2006.

[68] B. Saccà, R. Meyer, C. M. Niemeyer, Nature protocols, 2009, 4(3), 271-285.

[69] J. Kypr, I. Kejnovská, D. Renciuk, M. Vorlícková, Nucleic Acids Research 2009, 37(6), 1713 1725.

[70] M. Vorliččková, I. Kejnovská, K. Bednářová, D. Renčiuk, J. Kypr, Chirality 2012, 24(9), 691698.

[71] G. Reid Bishop and J. B. Chaires, Curr. Protoc. Nucleic Acid Chem, edited by Serge L. Beaucage ... [et al.] 2002, 7.11.1.

[72] C. M. Castleberry, L. P. Rodicio, P. A. Limbach, Curr. Protoc. Nucleic Acid Chem., edited by Serge L. Beaucage ... [et al.] 2008, Chapter 10, Unit 10.2. 
[73] Kenneth A. Browne, Gong-Xin He, and Thomas C. Brice, J. Am. Chem. Soc. 1993, 115, 1012-1019.

[74] David C. Gale, David R. Goodlett, Karen J. Light-Wahl, and Richard D. Smith, J. Am. Chem. Soc. 1994, 116, 6027-6028.

[75] M. Gilar, Analytical biochemistry 2001, 298(2), 196-206.

[76] T. R. Cook, P. J. Stang, Chem.Rev. 2015, 115(15), 7001-7045.

[77] S. De, K. Mahata, M. Schmittel, Chem. Soc. Rev., 2010, 39(5), 1555-1575.

[78] Y.-Z. Hu, C. Chamchoumis, J. S. Grebowicz, R. P. Thummel, Inorg. Chem. 2002, 41(8), 2296-2300.

[79] a) S. Shotwell, H. L. Ricks, J. G. Morton, M. Laskoski, J. Fiscus, M. D. Smith, K. D. Shimizu, H.-C. Zur Loye, U. H. Bunz, J. Organomet. Chem. 2003, 671(1-2), 43-51; b) J. E. Fiscus, S. Shotwell, R. C. Layland, R. E. Hipp, A. M. Goforth, C. T. Chapman, M. D. Smith, Bunz, Uwe H. F, H.-C. Zur Loye, J. Chem. Crystallogr. 2005, 35(11), 903-912.

[80] F. A. Pereira, T. Fallows, M. Frank, A. Chen, G. H. Clever, Z. anorg. allg. Chem. 2013, 639(8-9), 1598-1605.

[81] a) B. Champin, P. Mobian, J.-P. Sauvage, Chem. Soc. Rev., 2007, 36(2), 358-366; b) M. Fujita, K. Umemoto, M. Yoshizawa, N. Fujita, T. Kusukawa, K. Biradha, Chem. Commun. 2001(6), 509-518;

[82] M. Han, D. M. Engelhard, G. H. Clever, Chem. Soc. Rev., 2014, 43(6), 1848-1860.

[83] S. Freye, J. Hey, A. Torras-Galán, D. Stalke, R. Herbst-Irmer, M. John, G. H. Clever, Angew. Chem. Int. Ed. 2012, 51(9), 2191-2194.

[84] S. Freye, D. M. Engelhard, M. John, G. H. Clever, Chemistry 2013, 19(6), 2114-2121.

[85] K. Tanaka, M. Shionoya, J. Org. Chem. 1999, 64(14), 5002-5003.

[86] B. Jash, J. Neugebauer, J. Müller, Inorg. Chim. Acta 2016.

[87] Spartan'14, Irvine, CA, USA 2014.

[88] M. J. Cavaluzzi, P. N. Borer, Nucleic Acids Research 2004, 32(1), e13.

[89] a) L. R. Holman and D. O. Jordan, Biopolymers 1972, 11, 1661-1684; b) C.H. Zimmer, G. Luck, H. Fritzsche, and H. Thiebel, BiopolymersS 1971, 10, 441-463.

[90] H. Yang, F. Altvater, De Bruijn, A Dowine, C. K. McLaughlin, P. K. Lo, H. F. Sleiman, Angew. Chem. Int. Ed. 2011, 50(20), 4620-4623.

[91] T. Nguyen, A. Brewer, E. Stulz, Angew. Chem. Int. Ed. 2009, 48(11), 1974-1977.

[92] a) S. A. E. Marras, Selection of Fluorophore and Quencher Pairs for Fluorescent Nucleic Acid Hybridization Probes; b) S. A. E. Marras, F. R. Kramer and S. Tyagi, Nucleic Acids Research 2002, 30(21), e122.

[93] P. Ensslen, H.-A. Wagenknecht, Dalton transactions, 2015, 44(15), 6715-6718.

[94] P. Dauphin-Ducharme, F. Rosati, A. Greschner, De Bruijn, A Dowine, D. Salvatore, V. Toader, K. L. Lau, J. Mauzeroll, H. Sleiman, Langmuir, 2015, 31(5), 1850-1854.

[95] L.-A. Fendt, I. Bouamaied, S. Thöni, N. Amiot, E. Stulz, J. Am. Chem. Soc. 2007, 129(49), 15319-15329.

[96] G. Altenhoff, F. Glorius, Adv. Synth. Catal. 2004, 346(13-15), 1661-1664.

[97] G. M. Sheldrick, Acta Crystallogr. A, Found. Crystallogr., 2008, 64(Pt 1), 112-122.

[98] S. Saglam, A. Disli, Y. Erdogdu, M. K. Marchewka, N. Kanagathara, B. Bay, M. T. Güllüoğlu, Spectrochim. Acta A Mol. Biomol. Spectrosc. 2015, 135, 1011-1018.

[99] a) G. H. Clever, K. Polborn, T. Carell, Angew. Chem. Int. Ed. 2005, 44(44), 7204-7208; b) L.-A. Fendt, I. Bouamaied, S. Thöni, N. Amiot, E. Stulz, J. Am. Chem. Soc. 2007, 129(49), 15319-15329. 
[100] M. J. Cavaluzzi, P. N. Borer, Nucleic Acids Research 2004, 32(1), e13.

[101] M. J. Frisch, G. W. Trucks, H. B. Schlegel, G. E. Scuseria, M. A. Robb, J. R. Cheeseman, G. Scalmani, V. Barone, B. Mennucci, G. A. Petersson, H. Nakatsuji, M. Caricato, X. Li, H. P. Hratchian, A. F. Izmaylov, J. Bloino, G. Zheng, J. L. Sonnenberg, M. Hada, M. Ehara, K. Toyota, R. Fukuda, J. Hasegawa, M. Ishida, T. Nakajima, Y. Honda, O. Kitao, H. Nakai, T. Vreven, J. A. Montgomery, Jr, J. E. Peralta, F. Ogliaro, M. Bearpark, J. J. Heyd, E. Brothers, K. N. Kudin, V. N. Staroverov, R. Kobayashi, J. Normand, K. Raghavachari, A. Rendell, J. C. Burant, S. S. Iyengar, J. Tomasi, M. Cossi, N. Rega, J. M. Millam, M. Klene, J. E. Knox, J. B. Cross, V. Bakken, C. Adamo, J. Jaramillo, R. Gomperts, R. E. Stratmann, O. Yazyev, A. J. Austin, R. Cammi, C. Pomelli, J. W. Ochterski, R. L. Martin, K. Morokuma, V. G. Zakrzewski, G. A. Voth, P. Salvador, J. J. Dannenberg, S. Dapprich, A. D. Daniels, Ö. Farkas, J. B. Foresman, J. V. Ortiz, J. Cioslowski, and D. J. Fox. Gaussian, Gaussian 09, Revision A.02, 2009. 


\section{Acknowledgments}

I would like to thank in first place God, Who provide me His strength in all the moments of my life. All Honor, all glory to the Lord. "And we know that God works all things together for the good of those who love Him, who are called according to His purpose." Romans 8:28.

I thank my dear mother, for all the effort and sacrifices that she did in life to provide me with honesty and dignity a good education, better conditions than I had, that allowed me to be who I am now.

I would like to express my gratitude to my supervisor, Prof. Dr. Guido H. Clever in accepting me as a Ph.D. student and providing me the research topic here presented. I also thank him for the motivation and for teaching me how to develop independent research skills during my project.

I thank all the thesis committee members and members of the examination board: Prof. Dr. Franc Meyer, Prof. Dr. Sofi Elmroth, Dr. Breder, Jun-Prof. Demir, Prof.Dr. Schneider and Prof. Dr. Vana. I would like to thank Prof. Dr. Sofi Elmroth, Prof. Dr. Claudia Höbartner and Prof. Dr. Eugen Stulz for the time spent listening to my questions, the help and knowledge given to me through great discussions.

An special thanks to the PhD program Metal Sites in Biomolecules, Structures, Regulation and Mechanisms (IRTG 1422) for the financial support. My special thanks to Dr. Steininger, for all her kindness, friendship and help, not only concerning administrative subjects of IRTG.

I thank Prof. Dr Ivo Feussner and his group members for helping me with gel documentation. Thanks to Prof. Dr. Diederichsen for allowing me to use his facilities and equipments. To all members of AK Diederichsen for being so nice whenever I needed to use an equipment there. Special thanks to Harita, Oleg and Zeynep, who taught me the first steps of DNA synthesis. I also thank the group of Prof. Stulz in England because of receiving me so well during my stay in your laboratory and Dr. Mark Light for the X-ray services. I also would like to thank Prof. Renata Diniz and Dr. Inke Siewert for being examples of woman in science, Matthias Hesse and Martin Schlote for supplying the chemicals, Dr. Holm Frauendorf for his support in my mass measurements. 
I also would like to thank the members of Clever lab, former and current ones, for a such nice atmosphere and team work: Sabrina, Muxin, Ilker, Thorben, Marina, Marcel, Susanne, Rongmei, Eike, Rujin, Anex, Mark and Witold. Thanks Mark and Witold for their help reviewing my thesis. Thanks Susanne and Marcel for their nice atmosphere sharing the office and lab with me, for their friendship and support. Thanks Marcel for all TI services and crazy Spanish rock songs! Thanks Susi for all the "germanizing tips," and bring me food when I get night long in the DNA synthesizer! 


\section{Curriculum Vitae}

\section{Personal Information}

Birth date:

07.01.1984

Place of birth:

Belo Horizonte (Brazil)

Nationality:

Brazilian

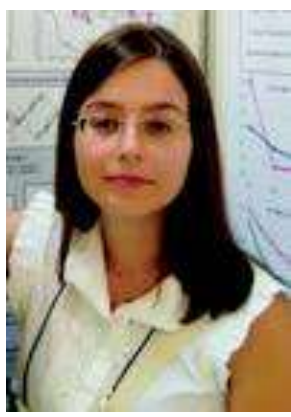

\section{Education}

05.2011- present

Georg-August-Universität, Göttingen, Germany

Ph.D. Student in IRTG 1422 International Research

Training Group, Metal Sites in Biomelecules:

Structures, Regulation and Mechanism.

Thesis realized in the group of Prof.Dr. Guido H.

Clever, Anorganische Chemie Institut

$03.2009-12.2010$

Federal University of Minas Gerais - Brazil

Master in Inorganic chemistry, emphasys for

bio-inorganic. CNPq scholarship, "level of

excellence (6-7) graduate student program“ CAPES.

$03.2003-07.2008$

Federal University of Juiz de Fora - Brazil

Bachelor in chemistry, FAPEMIG scholarship

for internship in research.

Publications:

F. A. Pereira, G. H. Clever, Inorg. Chim. Acta 2016, in press.

F. A. Pereira, T. Fallows, M. Frank, A. Chen, G.H. Clever, Z. Anorg. Allg. Chem. 2013, 639, (8-9), 1598-1605.

C. Demicheli, F. Frézard, F.A. Pereira, D.M. Santos, J.B. Magrum, N.P. Farrell, Journal of Inorg. Biochem. 2011, 105, 1753-1758. 